Supporting Information for

\title{
C(sp2)-H Bond Multiple Functionalization in Air for Construction of Tetrahydrocarbazoles with Continuous Quaternary Carbons and Polycyclic Diversification
}

Longlong Song, Dan Ni, Shikun Jia, Rou Pi, Suzhen Dong, Fan Yang, Jie Tang* and Shunying Liu*

Shanghai Engineering Research Center of Molecular Therapeutics and New Drug Development, School of Chemistry and Chemical Engineering, East China Normal University, 3663 North Zhongshan Road, Shanghai 200062, China

E-mail: jtang@chem.ecnu.edu.cn, syliu@sist.ecnu.edu.cn.

\section{Table of Contents}

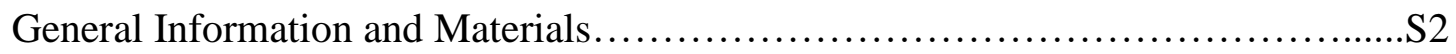

Experimental Procedures.................................................S214

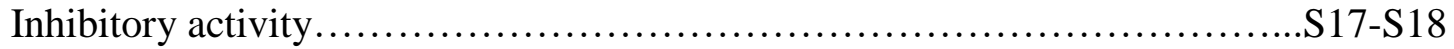

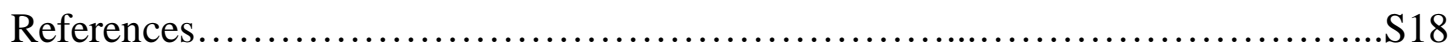

X-ray Diffraction Parameters and Data of $s y n-3 m$ and $s y n-5 e . . . \ldots \ldots \ldots \ldots . . . . S 19-S 20$

Characterization Data of Compounds ............................... S21-S43

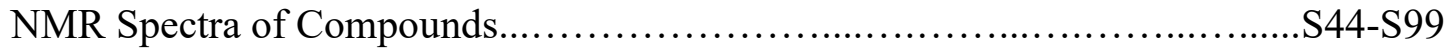




\section{General Information and Materials}

All ${ }^{1} \mathrm{H}$ NMR (400 MHz) and ${ }^{13} \mathrm{C}$ NMR (100 MHz) and ${ }^{19} \mathrm{~F}$ NMR (376 MHz) spectra were recorded on Brucker spectrometers in $\mathrm{CDCl}_{3}$. Tetramethylsilane (TMS) served as an internal standard $(\delta=0)$ for ${ }^{1} \mathrm{H}$ NMR, and $\mathrm{CDCl}_{3}$ was used as internal standard $(\delta=77.0)$ for ${ }^{13} \mathrm{C}$ NMR. Chemical shifts are reported in parts per million as follows: chemical shift, multiplicity $(\mathrm{s}=$ singlet, $\mathrm{d}=$ doublet, $\mathrm{t}=$ triplet, $\mathrm{q}=$ quartet, $\mathrm{m}$ $=$ multiplet, $\mathrm{br}=$ broad $).$ High-resolution mass spectrometry $($ HRMS $)$ was performed on IonSpec FT-ICR or Waters Micromass Q-TOF micro Synapt High Definition Mass Spectrometer. HPLC analysis was performed on Dalian Elite (UV230+ UV/Vis Detector and P230P High Pressure Pump). Melting points were uncorrected. Single crystal X-ray diffraction data (syn-3m, syn-5e) were recorded on Bruker-AXS SMART APEX II single crystal X-ray diffractometer. Yields for all compounds were combined yields for all isomers unless otherwise indicated.

All reactions and manipulations were carried out under an atmosphere in a flamedried or oven-dried flask containing magnetic stir bar. Dichloromethane (DCM), 1,2dichloroethane (DCE), $\mathrm{CHCl}_{3}$ and toluene was distilled over calcium hydride. Solvents for the column chromatography were distilled before use. $4 \AA$ molecular sieves was dried in a Muffle furnace at $250^{\circ} \mathrm{C}$ for $5 \mathrm{hrs}$.

\section{Experimental Procedures}

General procedure for contruction of tetrahydrospirocarbazoles (3):
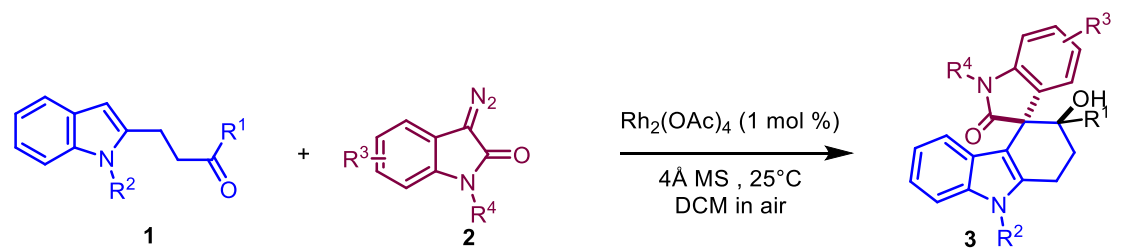

To a flask charged with $1 \mathrm{~mol} \% \mathrm{Rh}_{2}(\mathrm{OAc})_{4}$ and $4 \AA$ molecular sieves, indole derivative $1(0.2 \mathrm{mmol})$ in DCM (1 mL) was added directly to the flask. diazooxindole $2(0.4 \mathrm{mmol})$ in DCM $(1 \mathrm{~mL})$ were introduced by syringe pump over 1 hour at room temperature and the reaction solution was stirred for another 1 hour. After the 
completion of the reaction (monitored by TLC), the reaction mixture was filtrated and the filtrate was evaporated in vacuo to give the crude product. And then the crude product was purified by flash chromatography on silica gel (EtOAc / petroleum ether $=$ $1: 8 \sim 1: 2)$ to give the pure product.

\section{General procedure for construction of tetrahydrocarbazoles (5):}
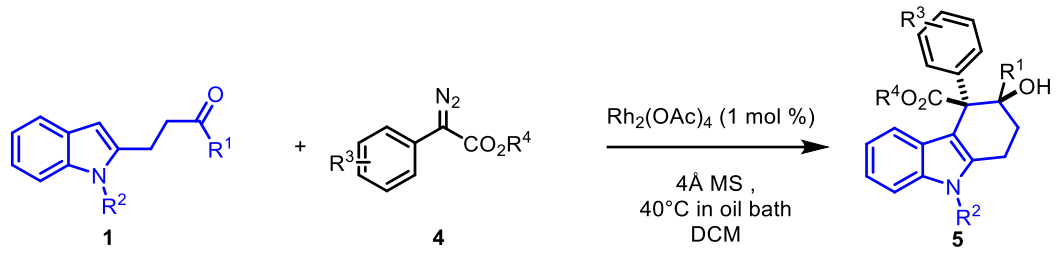

To a flask charged with $1 \mathrm{~mol} \% \mathrm{Rh}_{2}(\mathrm{OAc})_{4}$ and $4 \AA$ molecular sieves, indole derivative 1 (0.2 mmol) in DCM $(1 \mathrm{~mL})$ was added directly to the flask. phenyldiazoacetate $4(0.4 \mathrm{mmol})$ in DCM $(1 \mathrm{~mL})$ were introduced by syringe pump over 1 hour at $40{ }^{\circ} \mathrm{C}$ in oil bath and the reaction solution was stirred for another 1 hour. After the completion of the reaction (monitored by TLC), the reaction mixture was filtrated and the filtrate was evaporated in vacuo to give the crude product. And then the crude product was purified by flash chromatography on silica gel (EtOAc / petroleum ether $=1: 10 \sim 1: 4)$ to give the pure product.

\section{General procedure for preparation of Wittig Reagents:}
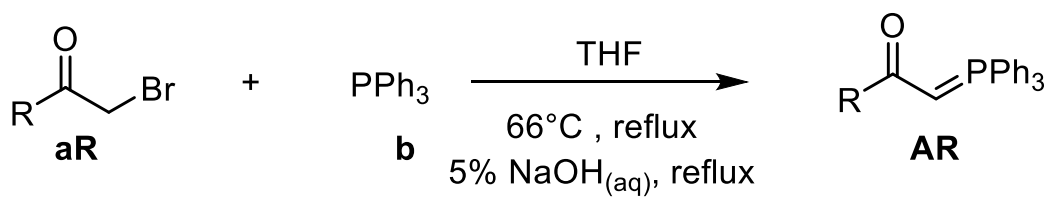

$$
\begin{aligned}
& \text { a R }=\mathrm{Ph} \\
& \text { b R }=4-\mathrm{CF}_{3} \mathrm{C}_{6} \mathrm{H}_{4} \\
& \text { c R }=4-\mathrm{OMeC}_{6} \mathrm{H}_{4} \\
& \text { d R }=4-\mathrm{CIC}_{6} \mathrm{H}_{4} \\
& \text { e R }=\mathrm{Me}
\end{aligned}
$$

\section{2-(triphenyl-15-phosphanylidene) ethan-1-one, (AR)}

Aryl-substituted 2-bromoethanone (5.00 mmol, 1.0 equiv.) and $\mathrm{PPh}_{3}(1.44 \mathrm{~g}, 5.50$ mmol, 1.1 equiv.) were refluxed in THF $(10 \mathrm{~mL})$ for $4 \mathrm{~h}$. Upon cooling, volatiles were removed, the solids redissolved in DCM and extracted with aq. $\mathrm{NaOH}(20 \%$ w/w in 
$\left.\mathrm{H}_{2} \mathrm{O}, 3 \times 20 \mathrm{~mL}\right)$, washed with water $(3 \times 20 \mathrm{~mL})$, dried over $\mathrm{MgSO}_{4}$, filtered and evaporated to dryness. The crude product was purified by silica gel chromatography ( 2 EA:1 PE) to afford the pure product. ${ }^{[1]}$

General Procedure for synthesis of $\beta-1 H$-indol ketones.<smiles>Cn1c(C=O)cc2ccccc21</smiles>

C

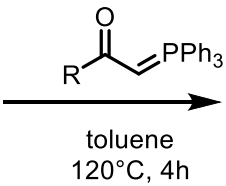

$120^{\circ} \mathrm{C}, 4 \mathrm{~h}$

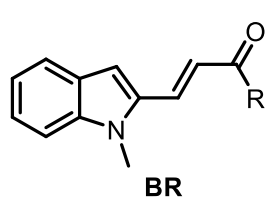

a $\mathrm{R}=\mathrm{Ph}$

b $\mathrm{R}=4-\mathrm{CF}_{3} \mathrm{C}_{6} \mathrm{H}_{4}$

c R $=4-\mathrm{OMeC}_{6} \mathrm{H}_{4}$

$\mathrm{d} \mathrm{R}=4-\mathrm{ClC}_{6} \mathrm{H}_{4}$

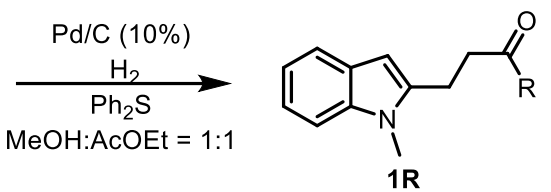

a $\mathrm{R}=\mathrm{Ph}$

b R $=4-\mathrm{CF}_{3} \mathrm{C}_{6} \mathrm{H}_{4}$ c R $=4-\mathrm{OMeC}_{6} \mathrm{H}_{4}$ $\mathrm{d} \mathrm{R}=4-\mathrm{ClC}_{6} \mathrm{H}_{4}$

1-Methyl-1H-indole-2-carbaldehyde, (C)

1-methyl-1H-indole (10g, $76 \mathrm{mmol})$ was dissolved in $\mathrm{Et}_{2} \mathrm{O}(50 \mathrm{~mL})$, then $n$-BuLi $(2.5 \mathrm{M}, 31 \mathrm{~mL}, 76 \mathrm{mmol})$ was added dropwise to the solution at $0^{\circ} \mathrm{C}$. The mixture was heated to reflux for $5 \mathrm{~h}$ followed by addition of DMF $(6.9 \mathrm{~mL}, 100 \mathrm{mmol})$ dropwise. The mixture was then reflux for $5 \mathrm{~h}$, which was monitored by TLC and quenched by the saturated solution of $\mathrm{NH}_{4} \mathrm{Cl}$ at ambient temperature. The extracts with ethyl acetate dried over anhydrous sodium sulfate and the solvent was removed under reduced pressre. The crude product was purified by column chromatography to give c (11 g, $85 \%$ ) as a yellow solid. ${ }^{[2]}$

\section{(E)-3-(1-methyl-1H-indol-2-yl)-1-phenylprop-2-en-1-one, (BR)}

1-methyl-1H-indole-2-carbaldehyde $(6.3 \mathrm{mmol})$ was dissolved in $20 \mathrm{~mL}$ of toluene. (Triphenylphosphoranylidene)-2-one $(9.4 \mathrm{mmol})$ was added and the mixture was heated at $120^{\circ} \mathrm{C}$ for $4 \mathrm{~h}$. After cooling, the solvent was removed in vacuo. Then the residue was poured into ether and filtered to remove $\mathrm{Ph}_{3} \mathrm{P}=\mathrm{O}$. The filtrate was concentrated and the residue was purified by column chromatography (petroleum ether: ethyl acetate $=20: 1$ to $8: 1)$ to give the product. ${ }^{[3]}$

\section{Procedure for the Chemoselective Hydrogenation of Olefins, (1R)}

After three vacuum / $\mathrm{H}_{2}$ cycles to replace air inside reaction tube with hydrogen, 
the mixture of the substrate $(5 \mathrm{mmol}), 10 \% \mathrm{Pd} / \mathrm{C}(10 \mathrm{wt} \%$ of the substrate), and diphenyl sulfide $(0.84 \mu \mathrm{L}, 5.00 \mu \mathrm{mol})$ in $\mathrm{MeOH}(5 \mathrm{~mL})$ and EtOAc $(5 \mathrm{~mL})$ was vigorously stirred at room temperature $\left(c a .25^{\circ} \mathrm{C}\right)$ under ordinary hydrogen pressure (balloon) for $6 \mathrm{~h}$. The reaction mixture was filtered using a diatomaceous earth filter $(0.45 \mu \mathrm{m})$, the filtrate was concentrated to provide the crude product. The residue was purified by silica column chromatography (PE / EtOAc $=100: 1$ to $50: 1)$ to give the product $($ yield $=99 \%) .{ }^{[4]}$

\section{General Procedure for Synthesis of 1-(1-methyl-1H-indol-2-yl) heptan-3-one}

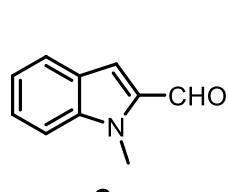

C

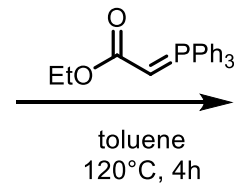<smiles>CCOC(=O)/C=C/c1cc2ccccc2n1C</smiles>

B5

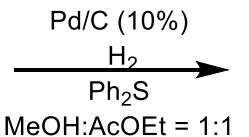

$\mathrm{MeOH}:$ AcOEt $=1: 1$

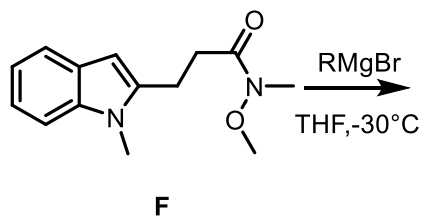

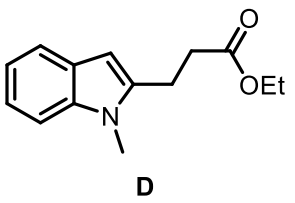

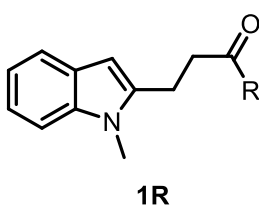

$\mathrm{e} R=\mathrm{Me}$

$f R=E t$ $\mathrm{g} \mathrm{R}=n-\mathrm{Bu}$

\section{Ethyl 3-(1-methyl-1H-indol-2-yl) acrylate, (B5)}

General procedure: 1-methyl- $1 H$-indole-2-carbaldehyde $(10 \mathrm{mmol})$ was dissolved in $20 \mathrm{~mL}$ of toluene. (Triphenylphosphoranylidene)-2-propanone (13 mmol) was added and the mixture was heated at $120^{\circ} \mathrm{C}$ for $4 \mathrm{~h}$. After cooling, the solvent was removed in vacuo. Then the residue was poured into ether and filtered to remove $\mathrm{Ph}_{3} \mathrm{P}=\mathrm{O}$. The filtrate was concentrated and the residue was purified by column chromatography (petroleum ether: ethyl acetate $=10: 1$ to $8: 1)$ to give the product $(2.1 \mathrm{~g}, 99 \%)$

\section{Ethyl 3-(1-methyl-1H-indol-2-yl) propynoate, (D)}

To the Ethyl 3-(1-methyl-1H-indol-2-yl) acrylate in $10 \mathrm{~mL}$ mixture solution $(\mathrm{MeOH}:$ EtOAc $=1: 1)$ was added 10 percent palladium on carbon $(50 \mathrm{mg}$ per $\mathrm{g}$ of substrate). The reaction mixture was flushed a few times with hydrogen and then stirred overnight at $\mathrm{rt}$ under a 1 atmosphere pressure of hydrogen. The reaction mixture was 
diluted with $\mathrm{CH}_{2} \mathrm{Cl}_{2}(2 \mathrm{~mL} / \mathrm{g})$ and was filtered through celite. The filtrate was concentrated to dryness to afford the title compound crude. the residue was purified by column chromatography (petroleum ether: ethyl acetate $=100: 1$ to $50: 1$ ) to give the product.

\section{3-(1-methyl-1H-indol-2-yl) propionic acid, (E)}

Sodium hydroxide (100mmol, 5.0 eq) was added to a methanol-dioxane (1:1) solution $(50 \mathrm{~mL})$ of Ethyl 3-(1-methyl-1H-indol-2-yl) propynoate $(20 \mathrm{mmol}, 1.0 \mathrm{eq})$, and the mixture was refluxed with heating for 2 hours. After evaporating the solvent, a 10 percent hydrochloric acid aqueous solution was added to the mixture until the aqueous layer became acidic, followed by extraction with ethyl acetate. The organic layer was dried over anhydrous sodium sulfate, and filtered, then the solvent was evaporated to obtain 3-(1-methy-1H-lindol-2-yl) propionic acid (5.7g, 82\%) as a colorless crystalline substance.

\section{3-(1-methyl-1H-indol-2-yl)- $N$-methoxy- $N$-methylpropionamide, (F)}

To a mixture of 3-(1-methyl-1H-indol-2-yl) propionic acid (14.8 mmol, $1.0 \mathrm{eq})$. O-dimethylhydroxylamine (17.3 mmol, $1.2 \mathrm{eq})$ and DMAP (1.5 mmol, $0.1 \mathrm{eq})$ in $\mathrm{CH}_{2} \mathrm{Cl}_{2}$. (50mL), $\mathrm{NEt}_{3}(17.3 \mathrm{mmol}, 1.2 \mathrm{eq})$ was added dropwise at $0^{\circ} \mathrm{C}$ and then DCC(17.3 mmol, $1.2 \mathrm{eq})$ was added. The reaction was stirred at $0^{\circ} \mathrm{C}$ for $15 \mathrm{~min}$, and then was allowed to warm to room temperature and stir for another 1 hour. The reaction was quenched with water. The solid was filtered off and washed with ethyl acetate. The filtrate was extracted with ethyl acetate for two times, and the combined organic layer was washed with brine and dried with anhydrous $\mathrm{Na}_{2} \mathrm{SO}_{4}$. After removal of the solvent, the residue was purified by silica chromatography (PE/EtOAc $=5: 1$ to $3: 1)$ to afford 3(1-methyl-1H-indol-2-yl)-N-methoxy-N-methylpropionamide (4.2 g, 67\%) as a white solid.

To a solution of 3-(1-methyl-1H-indol-2-yl)- $N$-methoxy- $N$-methylpropionamide (4 mmol, $1.0 \mathrm{eq})$ in THF $(20 \mathrm{~mL})$ methyl Grignard reagent $(6 \mathrm{mmol}, 2.3 \mathrm{~mL}, 2.5 \mathrm{M}$ in 
THF) was added at $0^{\circ} \mathrm{C}$. After being stirred for overnight at $\mathrm{rt}$, the reaction was quenched with saturate $\mathrm{NH}_{4} \mathrm{Cl}$ solution. Then the resulting solution was extracted with ethyl acetate, washed with saturated brine solution and dried with anhydrous $\mathrm{Na}_{2} \mathrm{SO}_{4}$. After being filtrated and concentrated, the residue was purified by silica chromatography $(\mathrm{PE} / \mathrm{EtOAc}=10: 1)$ to give $\mathbf{1 R}($ yield $=60 \sim 85 \%)$ as a colorless liquid.

General Procedure for synthesis of 2a,5-dimethyl-9c-phenyl-3,4,5,9ctetrahydrooxeto[2,3-c]carbazol-1(2aH)-one (5ab):
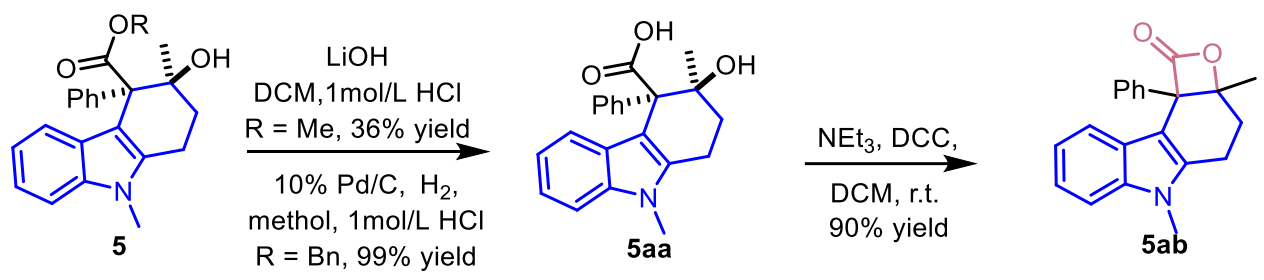

In a $25-\mathrm{mL}$ round-bottom flask were placed $\mathbf{5 m}(1.00 \mathrm{mmol})$ and $10 \% \mathrm{Pd} / \mathrm{C}(10$ wt $\%$ of the substrate) in menthol $(20 \mathrm{~mL})$, and the mixture was stirred at room temperature under a hydrogen atmosphere (balloon) for $8 \mathrm{~h}$ was added, and the mixture was passed through a membrane filter to remove the catalyst. The filtrate was concentrated in vacuo and was acidized with $1 \mathrm{M} \mathrm{HCl}$, extracted with methylene chloride to give the pure product 5aa.

In a $25-\mathrm{mL}$ round-bottom flask were placed $\mathbf{5 s}(1.00 \mathrm{mmol})$ and $\mathrm{LiOH}(1.4 \mathrm{mmol})$ in DCM $(10 \mathrm{~mL})$, and the mixture was stirred at room temperature for $3 \mathrm{~h}$, the mixture was neutralized with $1 \mathrm{M} \mathrm{HCl}$ extracted with methylene chloride. Purification by column chromatography to give the pure product 5aa.

Carboxylic acid 5aa $(0.2 \mathrm{mmol})$ was dissolved in dichloromethane $(4 \mathrm{~mL})$ under argon and cooled to $0^{\circ} \mathrm{C}$. Triethylamine $(0.3 \mathrm{mmol})$, DCC $(0.3 \mathrm{mmol})$ and 3-[chloro- $(2-$ oxooxazolidin-3-yl)phosphoryl]oxazolidin-2-one $(0.3 \mathrm{mmol})$ were added, and the mixture was stirred at rt. . the mixture was extracted with dichloromethane after 2 hrs. and washed with brine, the combined organic phases were dried with $\mathrm{Na}_{2} \mathrm{SO}_{4}$, filtered and concentrated. Purification by column chromatography to yield 5ab. 
General Procedure for synthesis of methyl 12-hydroxy-2-methoxy-6,12-dimethyl11-phenyl-6H-5a,10b-butanobenzofuro[2,3-b]indole-11-carboxylate (5bc):
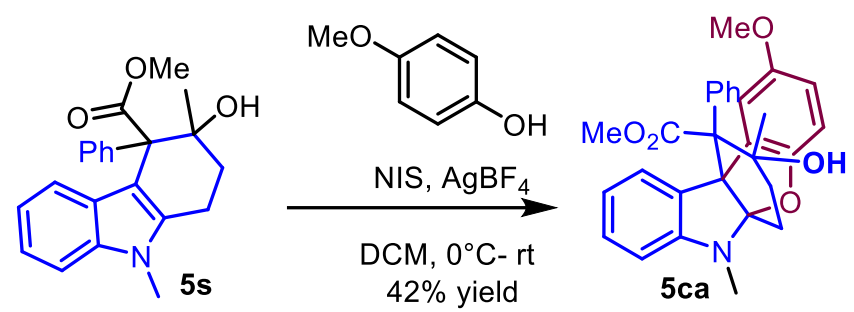

In a $10-\mathrm{mL}$ round-bottom flask were placed $\mathbf{5 s}(0.2 \mathrm{mmol}), \mathrm{AgBF}_{4}(0.02 \mathrm{mmol})$ and phenol $(0.8 \mathrm{mmol})$ in DCM $(5 \mathrm{~mL})$ at $\mathrm{N}_{2}$, NIS $(0.21 \mathrm{mmol})$ was added slowly to the mixture, then the mixture was stirred at room temperature for $0.8 \mathrm{~h}$, the reaction was quenched with saturate $\mathrm{NH}_{4} \mathrm{Cl}$ solution. Then the resulting solution was extracted with ethyl acetate, washed with saturated brine solution and dried with anhydrous $\mathrm{Na}_{2} \mathrm{SO}_{4}$. After being filtrated and concentrated, the residue was purified by silica chromatography.

General Procedure for synthesis of methyl 9-methyl-3-methylene-4-oxo-10phenyl-1,2,3,4-tetrahydro-9H-4a,9a-methanocarbazole-10-carboxylate (5ca):<smiles>C=C1CCc2c(c3ccccc3n2C)C1=O</smiles>

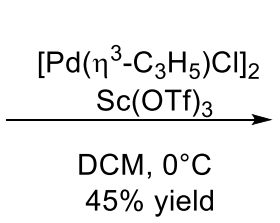<smiles>COC(=O)C12CCC(N(C)c3ccccc3C1=O)C2(OC)OC</smiles>

In a $10-\mathrm{mL}$ flask were placed $5 \mathbf{d}(0.2 \mathrm{mmol}),\left[\mathrm{Pd}\left(\eta-\mathrm{C}_{3} \mathrm{H}_{5}\right) \mathrm{Cl}\right]_{2}(1 \mathrm{~mol} \%)$ and $\mathrm{Sc}(\mathrm{OTf})_{3}(10 \mathrm{~mol} \%)$ in DCM $(1 \mathrm{~mL})$ at $\mathrm{N}_{2}, 4 a(0.21 \mathrm{mmol})$ was added slowly to the mixture, then the mixture was stirred at $0^{\circ} \mathrm{C}$ for $1 \mathrm{~h}$, the reaction was quenched with saturate $\mathrm{NH}_{4} \mathrm{Cl}$ solution. Then the resulting solution was extracted with ethyl acetate, washed with saturated brine solution and dried with anhydrous $\mathrm{Na}_{2} \mathrm{SO}_{4}$. After being filtrated and concentrated, the residue was purified by silica chromatography. 
Total synthesis of Ondansetron (5de):

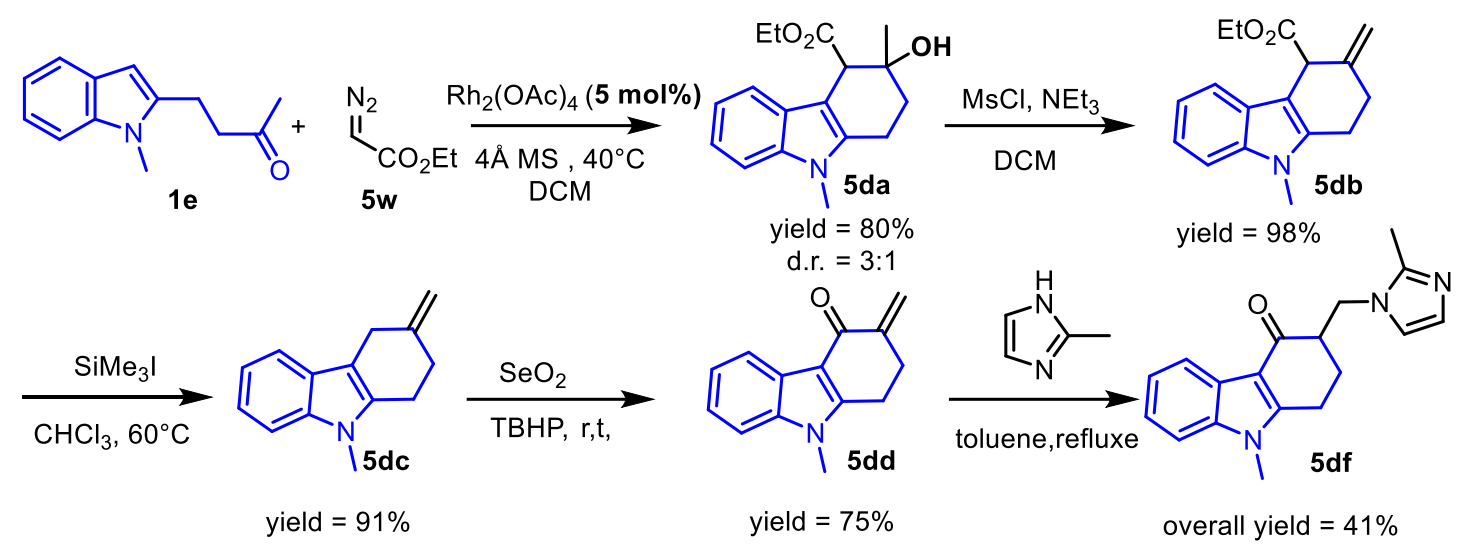

To a flask charged with $1 \mathrm{~mol} \% \mathrm{Rh}_{2}(\mathrm{OAc})_{4}$ and $4 \AA$ molecular sieves, indole derivative 1e (1 mmol) in DCM $(4 \mathrm{~mL})$ was added directly to the flask. ethyl diazoacetate $5 \mathbf{w}(2 \mathrm{mmol})$ in DCM $(3 \mathrm{~mL})$ were introduced by syringe pump over 2 hours at $40^{\circ} \mathrm{C}$ and the reaction solution was stirred for another 1 hour. After the completion of the reaction (monitored by TLC), the reaction mixture was filtrated and the filtrate was evaporated in vacuo to give the crude product 5da. Then the crude product was used directly without purification.

To a flask charged with $\mathbf{5 d a}(1.0$ eq. $)$ in DCM $(5 \mathrm{~mL})$ at $0^{\circ} \mathrm{C}$, triethylamine (2.0 eq.) was added into the mixture, and methane sulfonyl chloride (3.0 eq.) was added slowly. After return to room temperature and stirred for overnight at the same temperature. After the completion of the reaction (monitored by TLC), washed with saturated brine solution, extracted with DCM. and dried with anhydrous $\mathrm{Na}_{2} \mathrm{SO}_{4}$. After being filtrated and concentrated, the residue was purified by flash silica chromatography to yield the product $5 \mathbf{d b}$.

5db $(3 \mathrm{mmol})$ and trimethylsilyliodide $(0.4 \mathrm{ml} ; 3.2 \mathrm{mmol})$ are dissolved in $\mathrm{CHCl}_{3}(4 \mathrm{ml})$ and heated at $60{ }^{\circ} \mathrm{C}$ for $1 \mathrm{~h}$. After cooling to room temperature, the mixture is acidified with $1 \mathrm{M} \mathrm{HCl}$ and the water phase washed with DCM. The combined aqueous phases are treated with a saturated $\mathrm{Na}_{2} \mathrm{CO}_{3}$ solution and extracted with methylene chloride. The combined organic phases are washed with a saturated brine solution, dried over $\mathrm{Na}_{2} \mathrm{SO}_{4}$, filtered and evaporated. Then the crude product $5 \mathbf{d c}$ 
was used directly without purification.

Selenium dioxide (300 mg, $3 \mathrm{mmol}$ ) was dissolved in $10 \mathrm{ml}$ of $\mathrm{DCM}$ at $0^{\circ} \mathrm{C}$ and under an argon atmosphere was added t-butylhydroperoxide (4M, $1 \mathrm{~mL}, 3.5 \mathrm{mmol})$, stirred for 30 minutes, a solution of compound $\mathbf{5 d c}(2 \mathrm{mmol})$ in dichloromethane $(1 \mathrm{~mL})$ was slowly added dropwise. After rise to room temperature, stirring overnight. The reaction mixture was quenched with saturated aqueous sodium thiosulfate solution and extracted with DCM. The organic layer was washed with saturated aqueous solution of $\mathrm{Na}_{2} \mathrm{CO}_{3}$, water and saturated brine, and dried over anhydrous $\mathrm{Na}_{2} \mathrm{SO}_{4}$. The crude product obtained by filtration and purification was purified on a silica gel column (eluent: PE / EA = 5:1 2:1) to give a colorless solid 5dd.

The $5 \mathbf{d d}(0.3 \mathrm{mmol})$ and 2-methylimidazole $(0.5 \mathrm{mmol})$ were added to toluene $(10$ $\mathrm{mL}$ ), and the mixture was heated to reflux for 2 hours. The reaction was cooled to room temperature and washed with saturated brine solution, extracted with dichloromethane. and dried with anhydrous $\mathrm{Na}_{2} \mathrm{SO}_{4}$. After being filtrated and concentrated, the residue was purified by flash silica chromatography to yield the product Ondansetron 5de. 
Table S1 The exploration of enantioselective reaction.
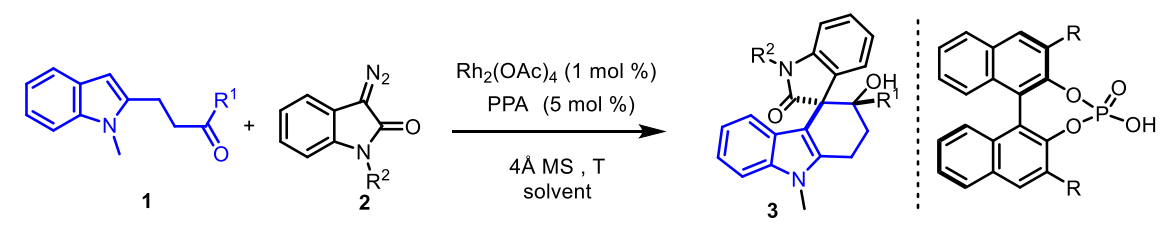

PA-1a: $\mathrm{R}=\mathrm{H}$

PA-1b: $\mathrm{R}=2,4,6-\mathrm{Pr}_{3} \mathrm{C}_{6} \mathrm{H}_{2}$

PA-1c: $\mathrm{R}=3,5-\mathrm{CF}_{3} \mathrm{C}_{6} \mathrm{H}_{3}$

PA-1d: $\mathrm{R}=\mathrm{SiPh}_{3}$

PA-1e: R= 9-Phenanthryl

\begin{tabular}{cccccccc}
\hline Entry & Solvent & PPA & $\mathrm{R}^{1}$ & $\mathrm{dr}$ & $\mathrm{T}^{\circ} \mathrm{C}$ & Yield (\%) & ee (\%) \\
\hline 1 & DCM & PA-1a & $\mathrm{CH}_{3}$ & $20: 1$ & 25 & 99 & 0 \\
$2^{\mathrm{b}}$ & DCM & PA-1b & $\mathrm{CH} 3$ & $10: 1$ & 25 & 88 & $15 / 45$ \\
3 & $\mathrm{CHCl}_{3}$ & PA-1c & $\mathrm{CH} 3$ & $15: 1$ & 25 & 85 & $<10$ \\
4 & DCM & PA-1d & $\mathrm{CH} 3$ & $15: 1$ & 25 & 90 & $<10$ \\
5 & DCM & PA-1e & $\mathrm{CH} 3$ & $15: 1$ & 25 & 76 & 0 \\
6 & DCM & PA-1b & $\mathrm{CH3}$ & $10: 1$ & 0 & 93 & $21 / 69$ \\
7 & THF & PA-1b & $\mathrm{CH} 3$ & $11: 1$ & 0 & 50 & $<10$ \\
8 & DCE & PA-1b & $\mathrm{CH} 3$ & $10: 1$ & 0 & 72 & $17 / 61$ \\
9 & DCM & PA-1b & Ph & $15: 1$ & 0 & 17 & 0 \\
10 & DCM & PA-1b & n-Bu & $10: 1$ & 0 & 50 & 4 \\
15 & DCM & PA-1b & CH3 & $10: 1$ & -10 & 84 & $26 / 88$ \\
\hline
\end{tabular}

Table S2 The exploration of enantioselective reaction.

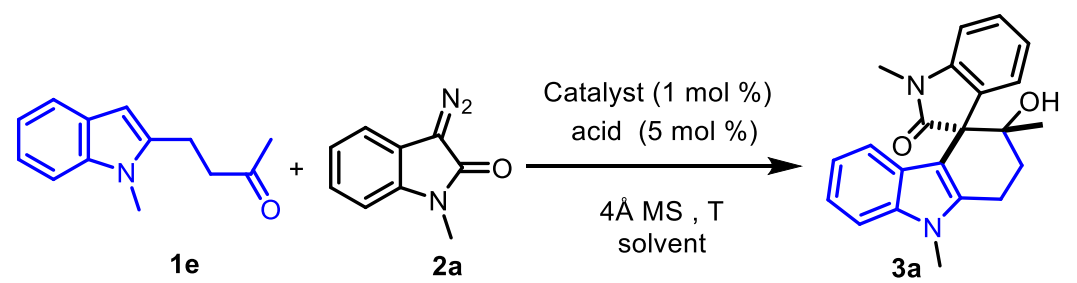

\begin{tabular}{ccccccc}
\hline Entry & Solvent & Catalyst & acid & $\mathrm{T}^{\circ} \mathrm{C}$ & Yield $\%$ & ee $\%$ \\
\hline 1 & $\mathrm{DCM}$ & {$[\mathrm{Rh}(\mathrm{COD}) \mathrm{Cl}]_{2}$} & diene & -10 & 50 & 0 \\
2 & $\mathrm{DCM}$ & $\mathrm{Ir}(\mathrm{cod})_{2} \mathrm{BF}_{4}$ & $\mathrm{PA}-1 \mathrm{~b}$ & -10 & 21 & 0 \\
3 & $\mathrm{DCM}$ & {$[\mathrm{Pd}(\mathrm{allyl}) \mathrm{Cl}]_{2}$} & $\mathrm{PA}-1 \mathrm{~b}$ & -10 & 22 & 0 \\
4 & $\mathrm{DCM}$ & {$\left[\mathrm{Rh}\left(\mathrm{C}_{2} \mathrm{H}_{4}\right)_{2} \mathrm{Cl}\right]_{2}$} & diene & -10 & 17 & 0 \\
\hline
\end{tabular}


Scheme S1 The exploration of enantioselective reaction.
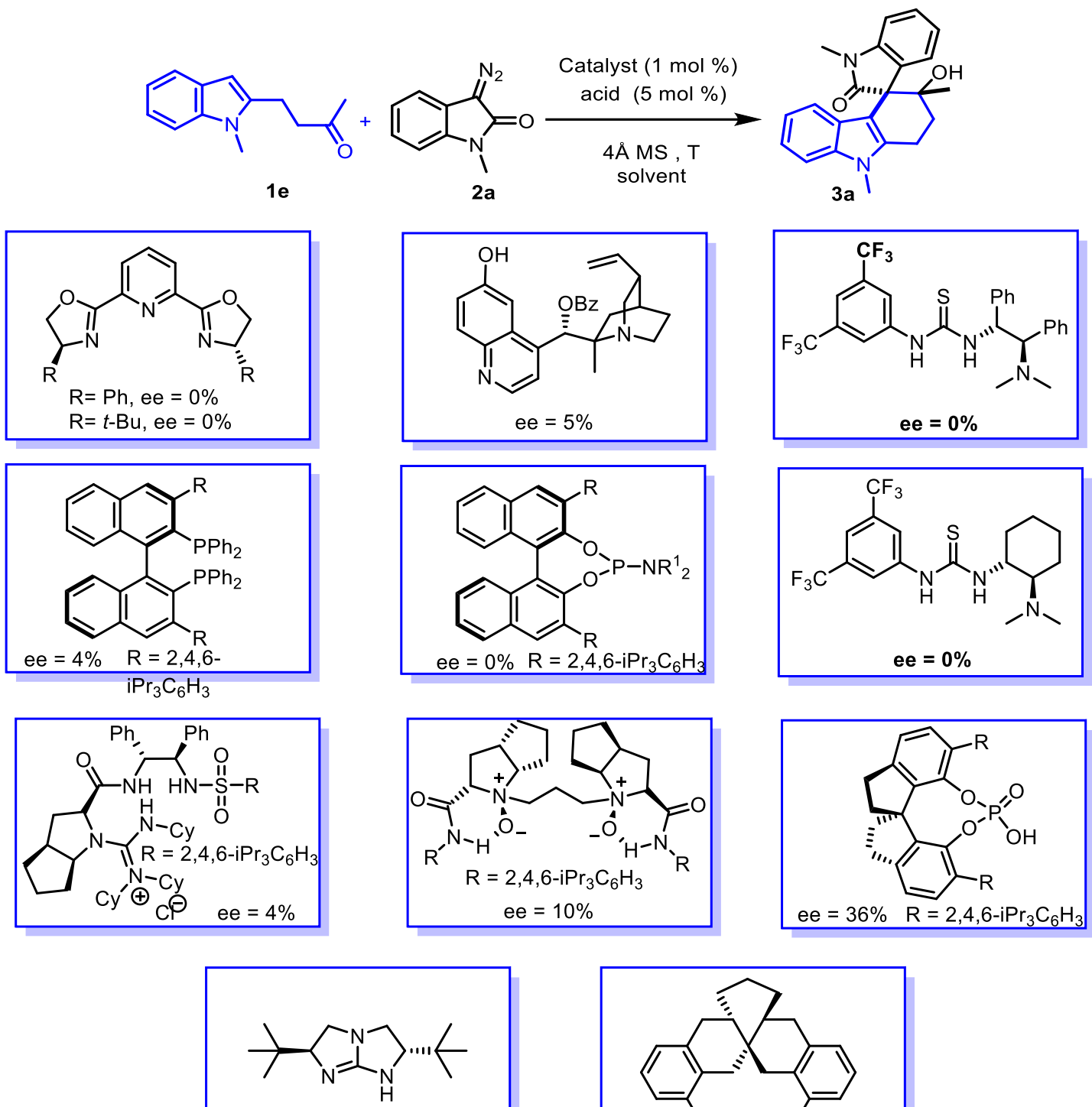

ee $=0 \%$

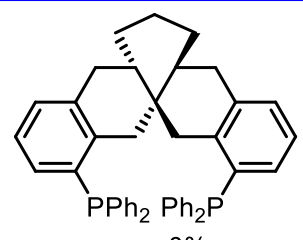

ee $=0 \%$ 
Scheme S2 Substrate scope for the tetrahydrocarbazole synthesis. ${ }^{[a]}$
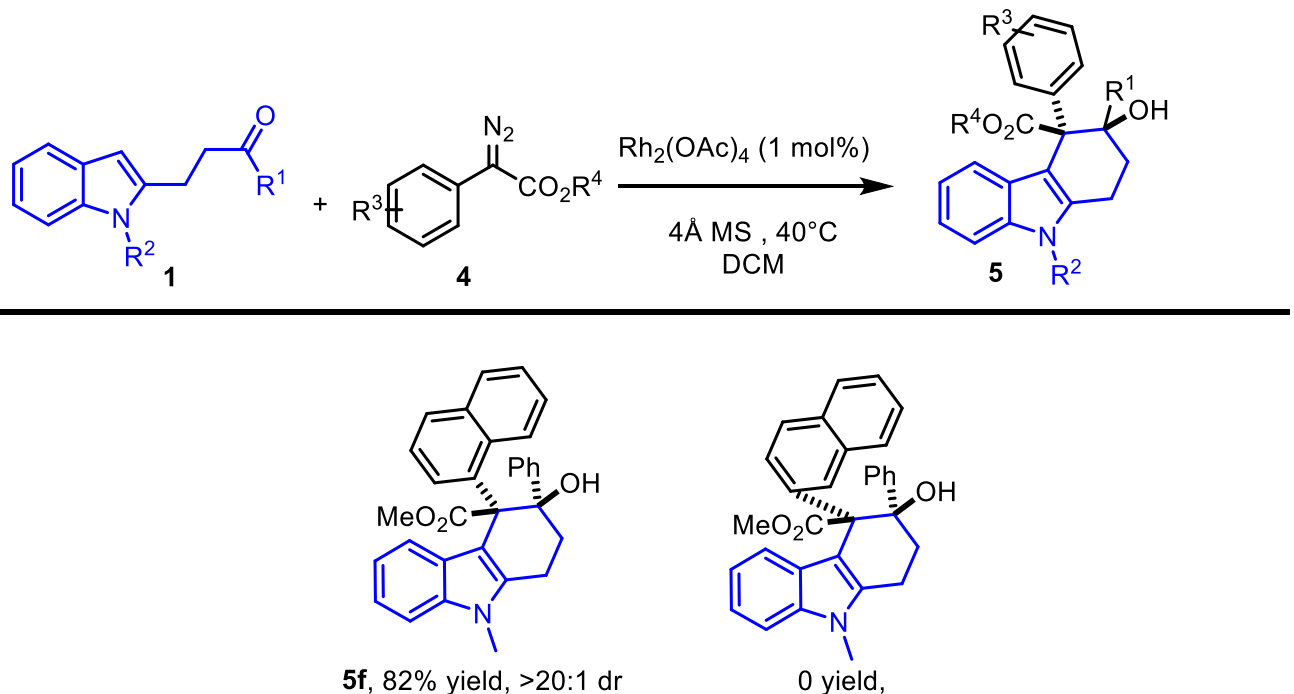

5f, $82 \%$ yield, $>20: 1 \mathrm{dr}$

[a] Reactions performed by adding $4(0.4 \mathrm{mmol})$ in $1 \mathrm{~mL}$ of DCM to the mixture of 1 $(0.2 \mathrm{mmol})$ and catalyst in $1 \mathrm{~mL}$ of DCM via syringe pump over 1 hour at $40^{\circ} \mathrm{C}$ in oil bath. 
Table S3 Optimization of reaction conditions. ${ }^{\text {[a] }}$

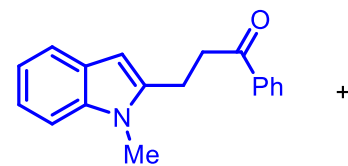

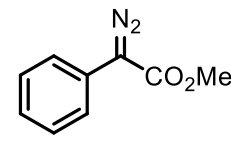

$4 a$

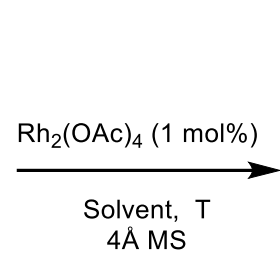

$4 \AA M S$

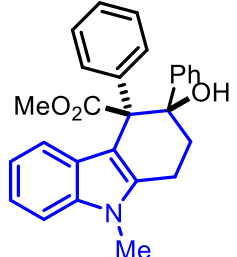

$5 s$

\begin{tabular}{ccccc}
\hline Entry & Solvent & $T\left({ }^{\circ} \mathrm{C}\right)$ & Yield $(\%)^{[\mathrm{b}]}$ & $\operatorname{dr}(\text { syn/anti })^{[\mathrm{c}]}$ \\
\hline 1 & DCM & r.t. & 83 & $95: 5$ \\
2 & DCM & 40 & 91 & $>20: 1$ \\
3 & DCM & 0 & 76 & $89: 11$ \\
4 & Toluene & 40 & 82 & $92: 8$ \\
5 & DCE & 40 & 77 & $90: 10$ \\
6 & $\mathrm{CHCl}_{3}$ & 40 & 85 & $93: 7$ \\
7 & $\mathrm{THF}^{2}$ & 40 & 62 & $90: 10$ \\
7 & $\mathrm{DCM}^{d}$ & 40 & 63 & $90: 10$ \\
\hline
\end{tabular}

[a] Reactions performed by adding $2(0.05 \mathrm{mmol})$ in $1 \mathrm{~mL}$ of solvent to the mixture of $1(0.1 \mathrm{mmol})$ and catalyst in $1 \mathrm{~mL}$ of solvent via syringe pump over 1 hour. [b] Isolated yield. [c] Determined by ${ }^{1} \mathrm{H}$ NMR of the crude products. [d] r.t. = room temperature. [e] Without $4 \AA ̊$ molecular sieves in air. DCE: dichloroethane; DCM: dichloromethane. 
Scheme S3 Application of tetrahydrocarbazole product.

a)
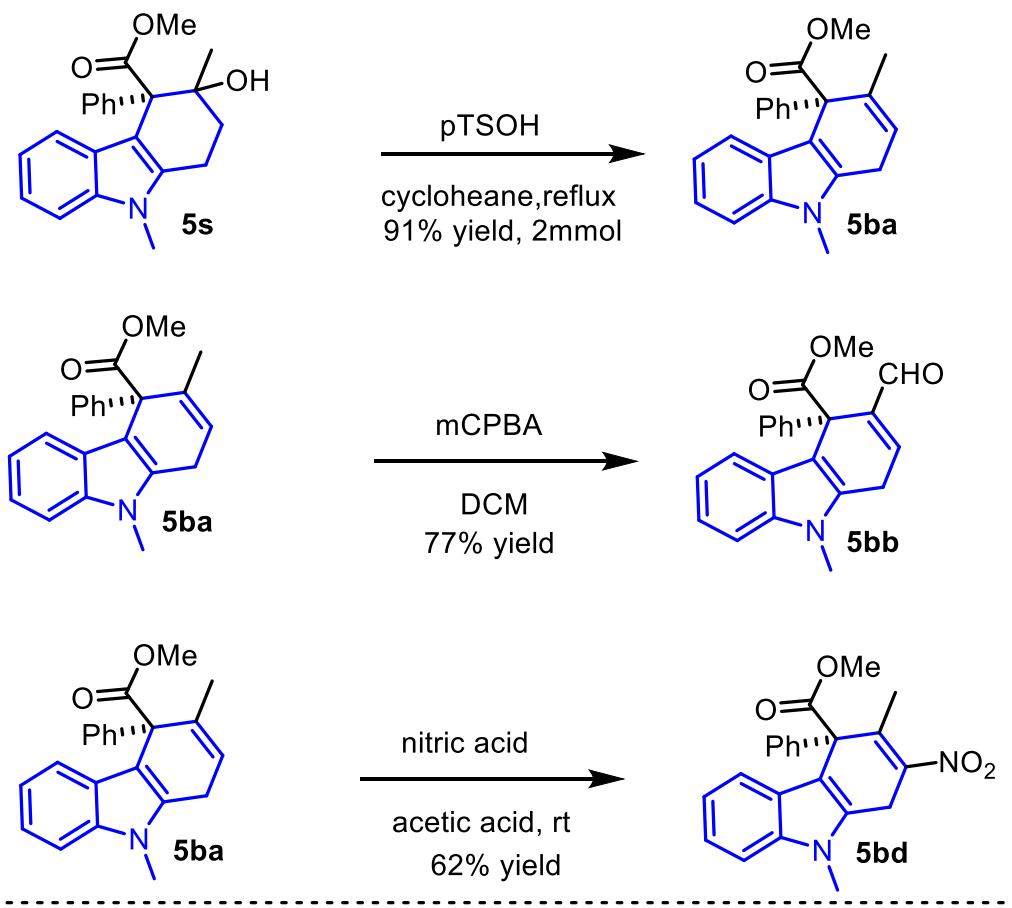

b)

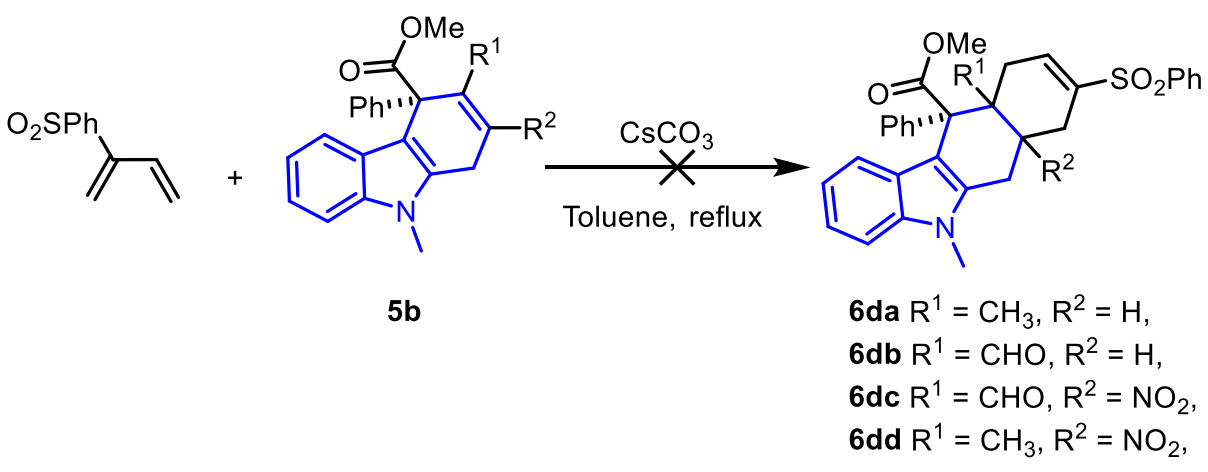

c)
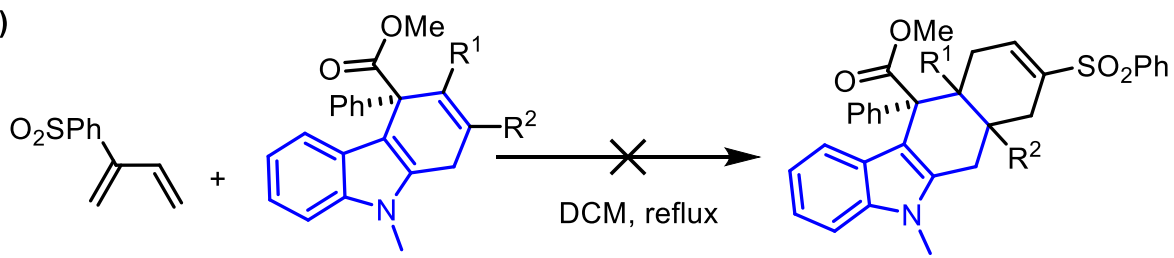

$5 b$

$$
\begin{aligned}
& \mathbf{6} \text { da } \mathrm{R}^{1}=\mathrm{CH}_{3}, \mathrm{R}^{2}=\mathrm{H}, \\
& \mathbf{6} \text { db } \mathrm{R}^{1}=\mathrm{CHO}, \mathrm{R}^{2}=\mathrm{H}, \\
& \mathbf{6} \text { dc } \mathrm{R}^{1}=\mathrm{CHO}, \mathrm{R}^{2}=\mathrm{NO}_{2}, \\
& \mathbf{6} \text { dd } \mathrm{R}^{1}=\mathrm{CH}_{3}, \mathrm{R}^{2}=\mathrm{NO}_{2},
\end{aligned}
$$




\section{Scheme S4 Gram-scale and control experiment.}

a) Gram-scale experiment<smiles>Cn1c(CCC(=O)[PH2+])cc2ccccc21</smiles>

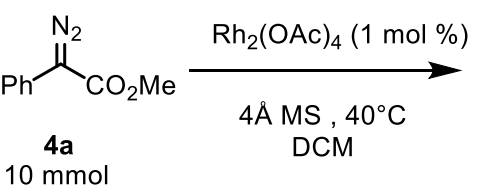

$10 \mathrm{mmol}$

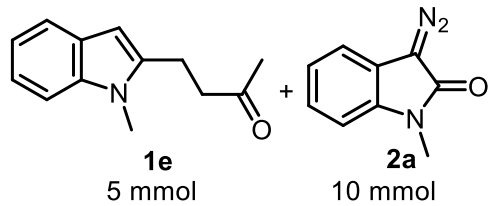

$\mathrm{Rh}_{2}(\mathrm{OAc})_{4}(1 \mathrm{~mol} \%)$

$4 \AA \mathrm{MS}, 25^{\circ} \mathrm{C}$ DCM
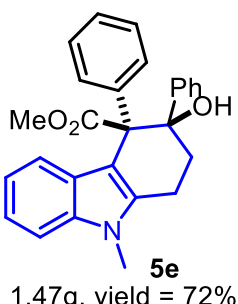

$1.47 \mathrm{~g}$, yield $=72 \%$

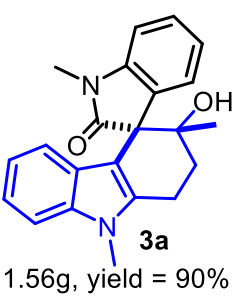

b) reaction of $1 \mathrm{~b}$ and $2 a$ in air

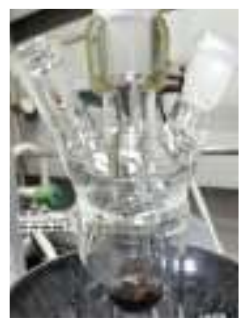

carried on in air at $25^{\circ} \mathrm{C}$

c) Control experiment<smiles>CC(=O)C(c1ccccc1)c1c(CCC(=O)c2ccccc2)c2ccccc2n1C</smiles>

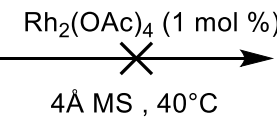

$$
\text { DCM }
$$

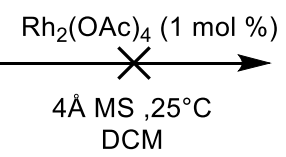

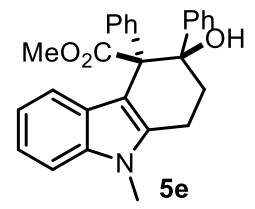

d) Stability of $5 e$

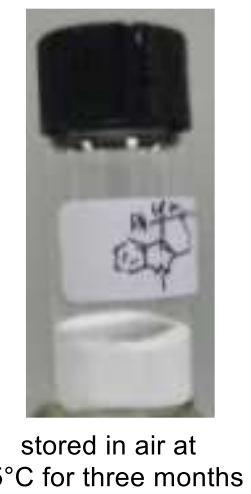

Gram-scale reaction of $\mathbf{1 a}$ with $\mathbf{4 a}$ was conducted to afford $\mathbf{5 e}$ in good yield (5.0 mmol scale, $1.47 \mathrm{~g}, 72 \%$ yield, Scheme S4a). Another gram-scale reaction of $\mathbf{1 b}$ with 2a was also proceeded affording 3a with satisfied results (5.0 mmol scale, $1.56 \mathrm{~g}, 90 \%$ ) (Scheme S4a). notably, this reaction was also conducted in air without productivities' reduction (Scheme $\mathrm{S} 4 \mathrm{~b}$ ). Besides, the resulting tetrahydrocarbazole products present a very good stability. For example, 5e was stored under room temperature for three months in air (Scheme S4d) and nearly no obvious degradation was observed by LCMS and ${ }^{1} \mathrm{H}-\mathrm{NMR}$ detection. The good stability of the products demonstrates the advantage of the tetrahydrocarbazole products used as intermediates for further diverse modification.

A number of primary mechanistic studies were conducted to understand this $\mathrm{C}-\mathrm{H}$ functionalized reaction pathway. Initially, $\mathrm{C}-\mathrm{H}$ insertion products $\mathbf{7 a}$ and $\mathbf{7 b}$ were prepared, and then reacted in succession under the criterial condition (Scheme S4c). No 
desired tetrahydrocarbazole products were observed which excludes a stepwise $\mathrm{C}-\mathrm{H}$ insertion/aldol addition pathway and verifies a direct trapping pathway of a zwitterioinc intermediate.

Table S4 Inhibitory activity of the representative resulting compounds against MG63, HOS, 143B and MNNG/HOS cell lines. proliferation present as inhibition (\%). ${ }^{[a]}$

\begin{tabular}{|c|c|c|c|c|c|}
\hline \multirow{2}{*}{\multicolumn{2}{|c|}{ Entry }} & \multicolumn{4}{|c|}{ Inhibition $(\%)$} \\
\hline & & \multirow{2}{*}{$\frac{\text { MG63 }}{100.10 \pm 4.69}$} & \multirow{2}{*}{$\frac{\mathrm{MNNG} / \mathrm{HOS}}{95.90 \pm 2.08}$} & \multirow{2}{*}{$\frac{\mathrm{HOS}}{105.50 \pm 8.42}$} & \multirow{2}{*}{$\frac{143 \mathrm{~B}}{104.80 \pm 0.18}$} \\
\hline 1 & $3 a$ & & & & \\
\hline 2 & $3 b$ & $62.51 \pm 1.50$ & $61.50 \pm 0.59$ & $53.79 \pm 3.13$ & $73.60 \pm 1.28$ \\
\hline 3 & $3 c$ & $101.15 \pm 2.04$ & $98.90 \pm 3.25$ & $120.61 \pm 0.45$ & $115.31 \pm 3.95$ \\
\hline 4 & $3 d$ & $114.51 \pm 0.05$ & $99.52 \pm 1.17$ & $79.01 \pm 3.40$ & $69.17 \pm 8.71$ \\
\hline 5 & $3 \mathrm{e}$ & $103.94 \pm 3.54$ & $100.88 \pm 0.26$ & $103.54 \pm 3.04$ & $49.26 \pm 0.64$ \\
\hline 6 & $3 f$ & $102.95 \pm 1.17$ & $100.04 \pm 2.46$ & $120.27 \pm 0.77$ & $102.67 \pm 3.18$ \\
\hline 7 & $3 g$ & $99.16 \pm 0.56$ & $87.46 \pm 1.98$ & $120.67 \pm 4.02$ & $87.99 \pm 1.77$ \\
\hline 8 & $3 \mathrm{~h}$ & $25.88 \pm 3.41$ & $1.37 \pm 0.16$ & $4.01 \pm 0.41$ & $8.30 \pm 0.47$ \\
\hline 9 & $3 \mathrm{i}$ & $112.31 \pm 0.75$ & $95.54 \pm 2.08$ & $26.14 \pm 0.22$ & $49.26 \pm 0.64$ \\
\hline 10 & $3 \mathrm{j}$ & $92.17 \pm 4.43$ & $28.88 \pm 6.43$ & $52.88 \pm 1.65$ & $72.66 \pm 0.75$ \\
\hline 11 & $3 \mathrm{k}$ & $98.64 \pm 3.70$ & $78.35 \pm 1.17$ & $49.91 \pm 2.32$ & $41.77 \pm 2.30$ \\
\hline 12 & 31 & $97.71 \pm 0.70$ & $72.18 \pm 1.69$ & $59.72 \pm 1.34$ & $35.64 \pm 5.19$ \\
\hline 13 & $3 \mathrm{~m}$ & $101.54 \pm 2.95$ & $82.90 \pm 0.48$ & $75.87 \pm 0.15$ & $51.23 \pm 10.02$ \\
\hline 14 & 30 & $125.05 \pm 2.95$ & $111.73 \pm 1.41$ & $100.41 \pm 0.94$ & $90.70 \pm 0.88$ \\
\hline 15 & $3 p$ & $102.29 \pm 1.54$ & $94.72 \pm 2.70$ & ---- & ---- \\
\hline 16 & $5 a$ & $113.58 \pm 1.97$ & $83.67 \pm 1.25$ & $102.84 \pm 2.84$ & $100.00 \pm 2.12$ \\
\hline 17 & $5 b$ & $112.50 \pm 2.39$ & $82.06 \pm 2.54$ & $116.15 \pm 4.12$ & $75.11 \pm 2.77$ \\
\hline 18 & $5 c$ & $112.22 \pm 0.80$ & $97.66 \pm 0.08$ & $109.37 \pm 2.37$ & $88.62 \pm 1.00$ \\
\hline 19 & $5 d$ & $103.23 \pm 2.39$ & $63.63 \pm 1.21$ & $103.94 \pm 0.57$ & $78.20 \pm 1.83$ \\
\hline 20 & $5 e$ & $101.97 \pm 1.40$ & $94.96 \pm 4.40$ & $112.61 \pm 6.55$ & $75.41 \pm 2.71$ \\
\hline 21 & $5 f$ & $109.41 \pm 3.32$ & $95.48 \pm 1.53$ & $105.40 \pm 12.73$ & $115.96 \pm 8.19$ \\
\hline 22 & $5 \mathrm{~g}$ & $108.71 \pm 0.56$ & $76.33 \pm 0.60$ & $95.08 \pm 0.82$ & $86.79 \pm 3.95$ \\
\hline 23 & $5 \mathrm{~h}$ & $114.33 \pm 1.22$ & $71.69 \pm 0.00$ & $104.78 \pm 0.82$ & $72.61 \pm 1.71$ \\
\hline 24 & $5 \mathrm{i}$ & $95.69 \pm 0.84$ & $85.65 \pm 1.45$ & $108.02 \pm 2.01$ & $76.74 \pm 0.94$ \\
\hline 25 & $5 \mathrm{j}$ & $110.58 \pm 2.43$ & $88.55 \pm 1.13$ & $105.43 \pm 0.41$ & $70.74 \pm 3.54$ \\
\hline 26 & $5 \mathrm{k}$ & $100.80 \pm 3.70$ & $88.67 \pm 2.54$ & $111.99 \pm 0.52$ & $97.17 \pm 3.07$ \\
\hline 27 & 51 & $97.75 \pm 1.78$ & $89.15 \pm 2.86$ & $105.58 \pm 0.72$ & $86.24 \pm 1.06$ \\
\hline 28 & $5 n$ & $114.11 \pm 1.55$ & $105.19 \pm 0.55$ & $97.98 \pm 2.41$ & $79.39 \pm 1.22$ \\
\hline 29 & 50 & $118.05 \pm 3.29$ & $110.31 \pm 0.91$ & $100.82 \pm 0.98$ & $69.75 \pm 4.06$ \\
\hline 30 & $5 p$ & $99.95 \pm 2.39$ & $83.35 \pm 2.46$ & $116.48 \pm 0.88$ & $110.05 \pm 0.06$ \\
\hline 31 & $5 q$ & $100.51 \pm 1.36$ & $99.07 \pm 4.15$ & $113.16 \pm 0.62$ & $95.79 \pm 3.48$ \\
\hline 32 & $5 r$ & $92.84 \pm 0.61$ & $79.07 \pm 0.28$ & $109.92 \pm 0.26$ & $84.37 \pm 3.01$ \\
\hline
\end{tabular}




\begin{tabular}{cccccc}
33 & $5 \mathrm{~s}$ & $99.30 \pm 0.23$ & $88.02 \pm 0.52$ & $116.92 \pm 3.25$ & $104.00 \pm 0.00$ \\
34 & $5 \mathrm{t}$ & $107.40 \pm 0.47$ & $84.27 \pm 1.13$ & $123.92 \pm 6.75$ & $62.48 \pm 0.59$ \\
35 & $5 \mathrm{u}$ & $109.78 \pm 0.70$ & $87.54 \pm 1.90$ & $101.79 \pm 0.82$ & $97.29 \pm 5.13$ \\
35 & $5 \mathrm{v}$ & $62.01 \pm 5.78$ & $98.24 \pm 1.06$ & $55.91 \pm 0.85$ & $37.27 \pm 2.03$ \\
37 & $5 \mathrm{ab}$ & $108.42 \pm 2.94$ & $97.51 \pm 2.82$ & $104.84 \pm 1.92$ & $85.92 \pm 8.01$ \\
38 & $5 \mathrm{ba}$ & $97.89 \pm 1.45$ & $84.19 \pm 2.34$ & $113.34 \pm 3.93$ & $108.13 \pm 0.17$ \\
\hline
\end{tabular}

${ }^{\mathrm{a}}$ Inhibitions (\%) of HOS,143B, MG63 and MNNG/HOS were produced by the tested compounds at $100 \mu \mathrm{M}$.

\section{Reference:}

[1] C. Zhao, F. D. Toste, R. G. Bergman, J. Am. Chem. Soc. 2011, 133, 10787-10789.

[2] C. W. Zheng, Y. P. Lu, J. K. Zhang, X. K. Chen, Z. Chai, W. Y. Ma, G. Zhao, Chem. Eur. J. 2010, 16, $5853-5857$.

[3] T. Su, X. 1. Han, X. Y. Lu, Tetrahedron Letters. 2014, 55, 27-30.

[4] A. Mori et al. Tetrahedron. $2006,62,11925-11932$. 


\section{X-ray Diffraction Parameters and Data of syn-3m and syn-5e}
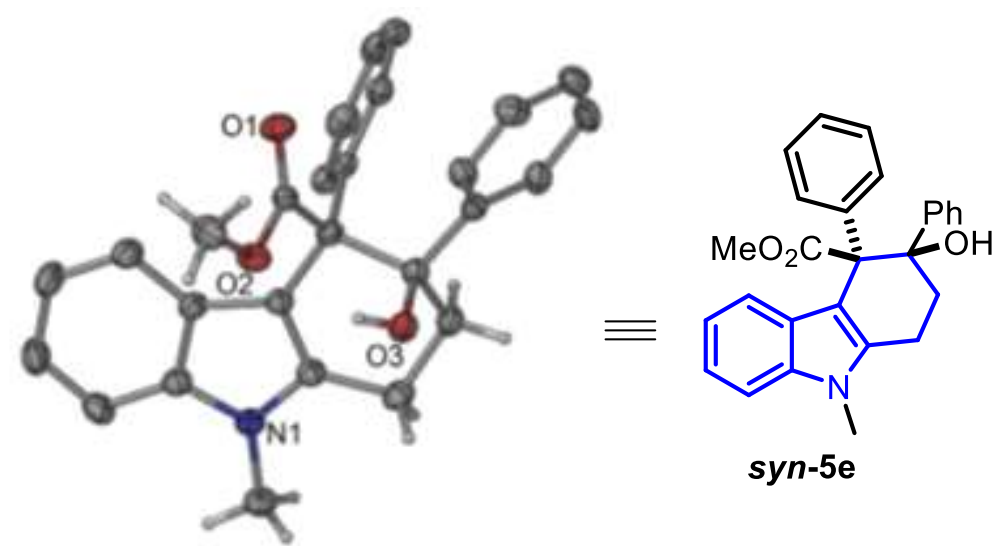

Bond precision: $\quad \mathrm{C}-\mathrm{C}=0.0030 \mathrm{~A} \quad$ Wavelength $=0.71073$

Cell:

$$
\mathrm{a}=12.2611(9)
$$

$\mathrm{b}=13.3296(9)$

alpha $=90$

beta $=99.021(3)$

$$
\mathrm{c}=12.7453(9)
$$

gamma $=90$

Temperature: $296 \mathrm{~K}$

Volume

Space group

Hall group

Moiety formula

Sum formula

$\mathrm{Mr}$

Dx, g cm-3

Z 44

$\mathrm{Mu}$ (mm-1)

F000

F000'

h,k, Imax

Nref

Tmin,Tmax

Tmin' 0.974

Calculated
2057.3(3)
P 21/n
-P 2yn
C27 H25 N O3
C27 H25 N O3
411.48

1.329

0.086

872.0

872.39

$14,15,15$

3623

$0.974,0.985$
Reported

2057.3(3)

$\mathrm{P} 2(1) / \mathrm{n}$

?

?

$\mathrm{C} 27 \mathrm{H} 25 \mathrm{~N} \mathrm{O} 3$

411.48

1.329

0.086

872.0

$14,15,15$

3623

$0.974,0.985$

Correction method $=$ \# Reported T Limits: Tmin $=0.974$ Tmax $=0.985$

AbsCorr $=$ MULTI-SCAN

Data completeness $=1.000$

$\operatorname{Theta}(\max )=25.010$

$\mathrm{R}($ reflections $)=0.0462(2602)$

$\mathrm{wR} 2($ reflections $)=0.1237(3623)$

$\mathrm{S}=1.018 \mathrm{Npar}=280$

Fig ure S1 The single crystal analysis for 5e (CCDC number: 1482623) 

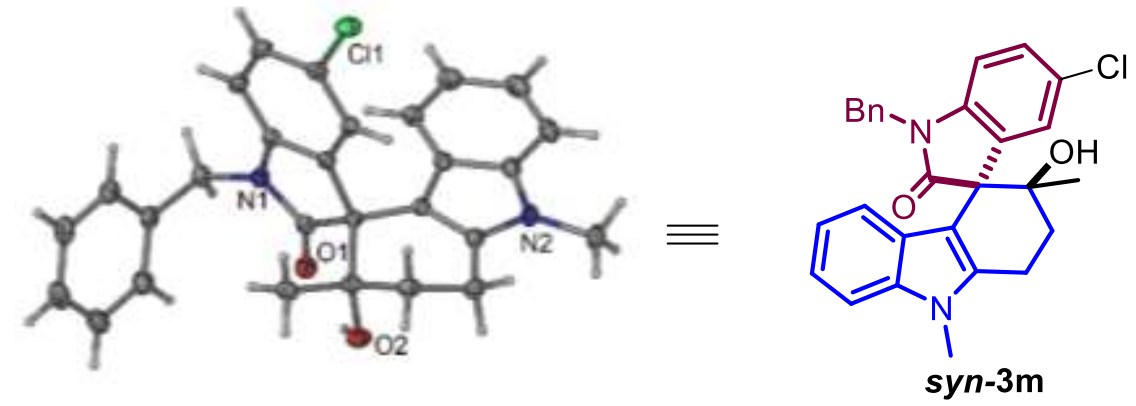

Bond precision:

$\mathrm{C}-\mathrm{C}=0.0023 \mathrm{~A}$

Wavelength $=1.54184$

Cell:

$\mathrm{a}=11.5136(1)$

$b=9.6782(1) \quad c=20.4295(2)$

alpha $=90$

beta $=94.605(1)$ gamma=90

Temperature:

$100 \mathrm{~K}$

Calculated

Reported

Volume

2269.13(4)

2269.13(4)

Space group

P 21/n

P 1 21/n 1

Hall group

-P 2yn

-P 2yn

Moiety formula

$\mathrm{C} 28 \mathrm{H} 25 \mathrm{Cl}$ N2 O2

$\mathrm{C} 28 \mathrm{H} 25 \mathrm{Cl} \mathrm{N} 2 \mathrm{O} 2$

Sum formula

$\mathrm{C} 28 \mathrm{H} 25 \mathrm{Cl} \mathrm{N} 2 \mathrm{O} 2$

$\mathrm{Mr}$

456.95

$\mathrm{C} 28 \mathrm{H} 25 \mathrm{Cl} \mathrm{N} 2 \mathrm{O} 2$

Dx,g cm-3

1.338

456.95

$\mathrm{Z}$

4

1.338

$\mathrm{Mu}$ (mm-1)

1.715

4

F000

960.0

1.715

F000'

963.99

h,k, Imax

$14,12,25$

960.0

Nref

4645

$14,11,25$

Tmin,Tmax

$0.781,0.872$

4600

Tmin'

0.521

$0.095,1.000$

Correction method $=$ \# Reported T Limits: Tmin $=0.095$ Tmax $=1.000$

AbsCorr $=$ MULTI-SCAN

Data completeness $=0.990$ Theta $(\max )=74.572$

$\mathrm{R}($ reflections $)=0.0417(4204)$ $w R 2($ reflections $)=0.1025(4600)$

$\mathrm{S}=1.056$

Npar $=302$

Figure S2 The single crystal analysis for 3m (CCDC number: 1958479) 


\section{Characterization Data of Compounds}

\section{3-(1-Methyl-1H-indol-2-yl)-1-phenylpropan-1-one (1a)}

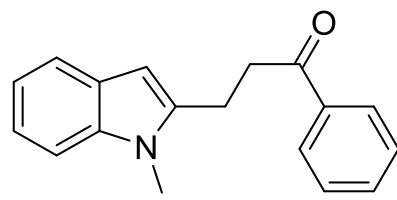

${ }^{1} \mathrm{H}$ NMR (400 MHz, Chloroform-d) $\delta 8.00(\mathrm{~d}, J=7.5 \mathrm{~Hz}$, 2H), $7.60-7.42(\mathrm{~m}, 4 \mathrm{H}), 7.30-7.24(\mathrm{~m}, 1 \mathrm{H}), 7.16(\mathrm{t}, J=$

$7.5 \mathrm{~Hz}, 1 \mathrm{H}), 7.06(\mathrm{t}, J=7.4 \mathrm{~Hz}, 1 \mathrm{H}), 6.28(\mathrm{~s}, 1 \mathrm{H}), 3.71(\mathrm{~s}$, $3 \mathrm{H}), 3.47-3.39$ (m, 2H), $3.23-3.14(\mathrm{~m}, 2 \mathrm{H}) .{ }^{13} \mathrm{C}$ NMR (100 MHz, Chloroform-d) $\delta$ $198.6,140.0,137.5,136.7,133.3,128.7,128.1,127.8,120.9,119.8,119.4,108.8$, 98.6,77.4, 77.1, 76.7, 37.4, 29.5, 21.1. HRMS(ESI) Calcd. for $\mathrm{C}_{18} \mathrm{H}_{17} \mathrm{NO} \mathrm{Na}(\mathrm{M}+\mathrm{Na})^{+}$ 286.1221, found: 286.1208 .

\section{3-(1-Methyl-1H-indol-2-yl)-1-(4-Methoxyphenyl) propan-1-one (1c)}

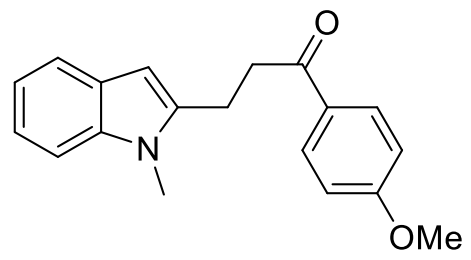

${ }^{1} \mathrm{H}$ NMR (400 MHz, Chloroform- $d$ ) $\delta 7.97$ (d, $J=8.9 \mathrm{~Hz}$, 2H), $7.52(\mathrm{~d}, J=7.8 \mathrm{~Hz}, 1 \mathrm{H}), 7.25(\mathrm{~d}, J=8.1 \mathrm{~Hz}, 1 \mathrm{H})$, $7.18-7.12(\mathrm{~m}, 1 \mathrm{H}), 7.08-7.03(\mathrm{~m}, 1 \mathrm{H}), 6.92(\mathrm{~d}, J=8.9$ $\mathrm{Hz}, 2 \mathrm{H}), 6.27(\mathrm{~s}, 1 \mathrm{H}), 3.84(\mathrm{~s}, 3 \mathrm{H}), 3.68(\mathrm{~s}, 3 \mathrm{H}), 3.38-$ $3.32(\mathrm{~m}, 2 \mathrm{H}), 3.20-3.13(\mathrm{~m}, 2 \mathrm{H}) .{ }^{13} \mathrm{C} \mathrm{NMR}\left(100 \mathrm{MHz}, \mathrm{CDCl}_{3}\right) \delta 197.2,163.7,140.2$, $137.5,130.3,129.9,127.9,120.8,119.8,119.4,113.9,108.9,98.5,55.5,37.0,29.5$, 21.2, 0.1. HRMS(ESI) Calcd. for $\mathrm{C}_{19} \mathrm{H}_{19} \mathrm{NO}_{2} \mathrm{Na}(\mathrm{M}+\mathrm{Na})^{+} 316.1310$, found: 316.1313 .

\section{3-(1-Methyl-1H-indol-2-yl)-1-(4-chlorophenyl) propan-1-one (1d)}

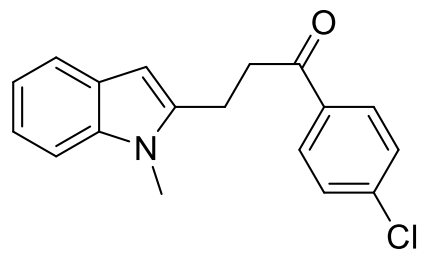

${ }^{1} \mathrm{H}$ NMR (400 MHz, Chloroform-d) $\delta 7.97-7.88$ (m, 2H), $7.52(\mathrm{dt}, J=7.8,1.0 \mathrm{~Hz}, 1 \mathrm{H}), 7.47-7.41(\mathrm{~m}, 2 \mathrm{H}), 7.27(\mathrm{~d}, J$ $=8.1 \mathrm{~Hz}, 1 \mathrm{H}), 7.19-7.13(\mathrm{~m}, 1 \mathrm{H}), 7.09-7.04(\mathrm{~m}, 1 \mathrm{H}), 6.27$ $(\mathrm{s}, 1 \mathrm{H}), 3.71(\mathrm{~s}, 3 \mathrm{H}), 3.42-3.37(\mathrm{~m}, 2 \mathrm{H}), 3.21-3.16(\mathrm{~m}$, 2H). ${ }^{13} \mathrm{C}$ NMR $\left(100 \mathrm{MHz}, \mathrm{CDCl}_{3}\right) \delta 197.4,139.8,139.7,137.4,135.0,129.5,129.0$, $127.8,120.9,119.9,119.4,108.9,98.6,37.4,29.5,21.0,0.0$.

HRMS(ESI) Calcd. for $\mathrm{C}_{18} \mathrm{H}_{16} \mathrm{ClNONa}(\mathrm{M}+\mathrm{Na})^{+} 320.0828$, found: 320.0818 . 


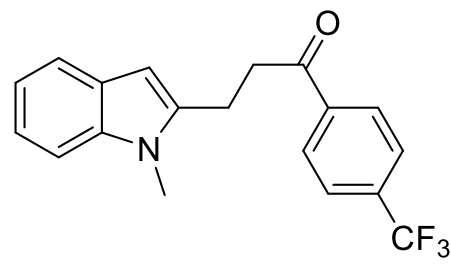

${ }^{1} \mathrm{H}$ NMR (400 MHz, Chloroform- $d$ ) $\delta 8.10(\mathrm{~d}, J=8.1 \mathrm{~Hz}$, 2H), $7.74(\mathrm{~d}, J=8.2 \mathrm{~Hz}, 2 \mathrm{H}), 7.53(\mathrm{~d}, J=7.8 \mathrm{~Hz}, 1 \mathrm{H})$, $7.28(\mathrm{~d}, J=8.1 \mathrm{~Hz}, 1 \mathrm{H}), 7.20-7.13(\mathrm{~m}, 1 \mathrm{H}), 7.12-7.03$

$3.26-3.17(\mathrm{~m}, 2 \mathrm{H}) .{ }^{13} \mathrm{C} \mathrm{NMR}\left(100 \mathrm{MHz}, \mathrm{CDCl}_{3}\right) \delta 197.6,139.5,139.3,134.6$ (q, $J=$ $32.7 \mathrm{~Hz}), 128.7,128.4,127.7,125.8$ (q, $J=3.7 \mathrm{~Hz}), 124.9,122.2,121.0,119.9,119.5$, 108.9, 98.6, 37.7, 29.5, 20.9, 0.0. ${ }^{19} \mathrm{~F}$ NMR (376 MHz, $\left.\mathrm{CDCl}_{3}\right) \delta-63.13 . \mathrm{HRMS}(\mathrm{ESI})$ Calcd. for $\mathrm{C}_{19} \mathrm{H}_{16} \mathrm{~F}_{3} \mathrm{NONa}(\mathrm{M}+\mathrm{Na})^{+}$354.1477, found: 354.1470 .

\section{4-(1-Methyl-1H-indol-2-yl) butan-2-one (1e)}

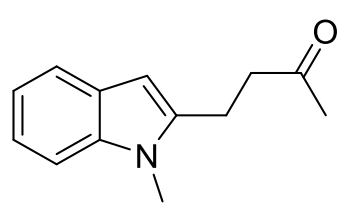

${ }^{1} \mathrm{H}$ NMR (400 MHz, Chloroform-d) $\delta 7.56-7.49$ (m, 1H), 7.29 - $7.25(\mathrm{~m}, 2 \mathrm{H}), 7.19-7.12(\mathrm{~m}, 1 \mathrm{H}), 7.10-7.03(\mathrm{~m}, 1 \mathrm{H}), 6.21$ $(\mathrm{d}, J=1.0 \mathrm{~Hz}, 1 \mathrm{H}), 3.69(\mathrm{~s}, 3 \mathrm{H}), 3.07-2.98(\mathrm{~m}, 2 \mathrm{H}), 2.95-$ 2.87 (m, 2H), 2.22 (s, 3H). $\left.{ }^{13} \mathrm{C} \mathrm{NMR} \mathrm{(100} \mathrm{MHz,} \mathrm{CDCl}_{3}\right) \delta 207.2,139.7,137.4,127.8$, 120.9, 119.9, 119.4, 108.9, 98.5, 42.1, 30.1, 29.4, 20.7, -0.0. HRMS(ESI) Calcd. for $\mathrm{C}_{14} \mathrm{H}_{17} \mathrm{NO}(\mathrm{M}+\mathrm{Na})^{+} 224.1057$, found: 224.1051 .

\section{3-(1-Methyl-1H-indol-2-yl)-1-ethylpropan-1-one (1f)}

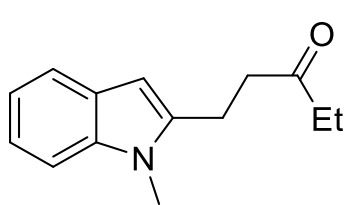

${ }^{1} \mathrm{H}$ NMR (400 MHz, Chloroform-d) $\delta 7.52(\mathrm{~d}, J=7.7 \mathrm{~Hz}, 1 \mathrm{H})$, $7.19-7.13(\mathrm{~m}, 1 \mathrm{H}), 7.09-7.03(\mathrm{~m}, 1 \mathrm{H}), 6.21(\mathrm{~s}, 1 \mathrm{H}), 3.69(\mathrm{~s}$, $3 \mathrm{H}), 3.07-3.00(\mathrm{~m}, 2 \mathrm{H}), 2.91-2.85(\mathrm{~m}, 2 \mathrm{H}), 2.50(\mathrm{q}, J=7.3$

$\mathrm{Hz}, 2 \mathrm{H}), 1.09$ (t, $J=7.3 \mathrm{~Hz}, 3 \mathrm{H}) .{ }^{13} \mathrm{C} \mathrm{NMR}\left(100 \mathrm{MHz}, \mathrm{CDCl}_{3}\right) \delta 210.0,139.8,137.4$, $127.8,120.8,119.8,119.3,108.8,98.4,40.8,36.2,29.5,20.7,7.8,0.0$. HRMS(ESI) Calcd. for $\mathrm{C}_{14} \mathrm{H}_{17} \mathrm{NO}(\mathrm{M}+\mathrm{Na})^{+} 238.1215$, found: 238.1208 .

\section{3-(1-Methyl-1H-indol-2-yl)-1-butylpropan-1-one (1g)}




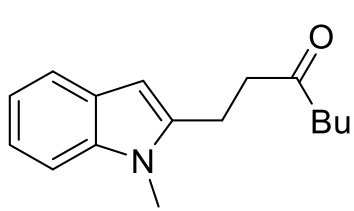

${ }^{1} \mathrm{H}$ NMR (400 MHz, Chloroform- $d$ ) $\delta 7.52(\mathrm{~d}, J=7.8 \mathrm{~Hz}, 1 \mathrm{H})$, $7.30-7.25(\mathrm{~m}, 1 \mathrm{H}), 7.20-7.12(\mathrm{~m}, 1 \mathrm{H}), 7.11-7.02(\mathrm{~m}, 1 \mathrm{H})$, $6.20(\mathrm{~s}, 1 \mathrm{H}), 3.68(\mathrm{~s}, 3 \mathrm{H}), 3.07-2.98(\mathrm{~m}, 2 \mathrm{H}), 2.91-2.82(\mathrm{~m}$, 2H), $2.47(\mathrm{t}, J=7.5 \mathrm{~Hz}, 2 \mathrm{H}), 1.66-1.56(\mathrm{~m}, 2 \mathrm{H}), 1.38-1.28(\mathrm{~m}, 2 \mathrm{H}), 0.91(\mathrm{t}, J=7.3$ $\mathrm{Hz}, 3 \mathrm{H}) .{ }^{13} \mathrm{C} \mathrm{NMR}\left(100 \mathrm{MHz}, \mathrm{CDCl}_{3}\right) \delta 209.7,139.9,137.4,127.8,120.8,119.8,119.3$, $108.8,98.4,42.8,41.2,29.5,26.0,22.4,20.7,13.9,0.0$.

HRMS(ESI) Calcd. for $\mathrm{C}_{16} \mathrm{H}_{21} \mathrm{NNaO}(\mathrm{M}+\mathrm{Na})^{+} 266.1515$, found: 266.1519 .

\section{3-(1-Benzyl-1H-indol-2-yl)-1-phenylpropan-1-one (1h)}

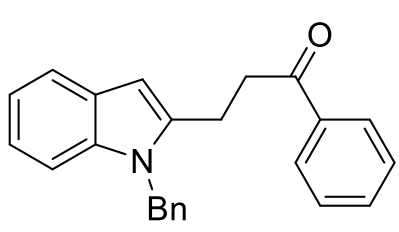

${ }^{1} \mathrm{H}$ NMR (400 MHz, Chloroform-d) $\delta 7.95-7.84$ (m, 2H), $7.61-7.50(\mathrm{~m}, 2 \mathrm{H}), 7.43(\mathrm{t}, J=7.6 \mathrm{~Hz}, 2 \mathrm{H}), 7.28-7.15(\mathrm{~m}$, $4 \mathrm{H}), 7.14-7.06(\mathrm{~m}, 2 \mathrm{H}), 7.01-6.91(\mathrm{~m}, 2 \mathrm{H}), 6.37(\mathrm{~s}, 1 \mathrm{H})$, 5.37 (s, 2H), $3.32(\mathrm{dd}, J=8.9,6.3 \mathrm{~Hz}, 2 \mathrm{H}), 3.13(\mathrm{dd}, J=9.0,6.1 \mathrm{~Hz}, 2 \mathrm{H}) .{ }^{13} \mathrm{C}$ NMR $\left(100 \mathrm{MHz}, \mathrm{CDCl}_{3}\right) \delta 198.5,140.0,137.8,137.4,136.7,133.2,128.8,128.7,128.0$, 127.3 126.0, 121.2, 120.0, 119.7, 109.4, 99.5, 46.5, 37.4, 21.0, 0.1. HRMS(ESI) Calcd. for $\mathrm{C}_{24} \mathrm{H}_{21} \mathrm{NONa}(\mathrm{M}+\mathrm{Na})^{+}$362.1534, found: 362.1521 .

3-hydroxy-1',3,9-trimethyl-1,2,3,9-tetrahydrospiro[carbazole-4,3'-indolin]-2'-one, (syn-3a):

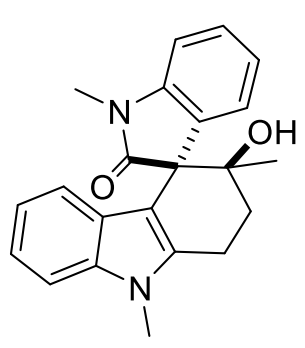

White solid, 99\% yield, ${ }^{1} \mathrm{H}$ NMR (400 MHz, Chloroform-d) $\delta 7.32$ $-7.26(\mathrm{~m}, 1 \mathrm{H}), 7.21(\mathrm{~d}, J=8.2 \mathrm{~Hz}, 1 \mathrm{H}), 7.08-6.92(\mathrm{~m}, 4 \mathrm{H}), 6.83$ $(\mathrm{t}, J=7.6 \mathrm{~Hz}, 1 \mathrm{H}), 6.58(\mathrm{~d}, J=8.0 \mathrm{~Hz}, 1 \mathrm{H}), 4.59(\mathrm{~s}, 1 \mathrm{H}), 3.68(\mathrm{~s}$, 3H), 3.47 (s, 3H), 3.22 (dt, $J=16.7,8.7 \mathrm{~Hz}, 1 \mathrm{H}), 2.89$ (d, $J=16.6$ $\mathrm{Hz}, 1 \mathrm{H}), 2.40-2.27(\mathrm{~m}, 2 \mathrm{H}), 1.02(\mathrm{~s}, 3 \mathrm{H}) .{ }^{13} \mathrm{C} \mathrm{NMR}(100 \mathrm{MHz}$, Chloroform-d) $\delta 179.7,143.3,137.3,136.7,132.7,128.1,125.2,125.0,123.1,120.8$, 119.2, 117.1, 108.9, 108.3, 73.0, 32.4, 29.3, 26.5, 25.1, 19.2. HRMS (ESI+): Calculated for $\mathrm{C}_{22} \mathrm{H}_{22} \mathrm{~N}_{2} \mathrm{O}_{2} \mathrm{Na}\left([\mathrm{M}+\mathrm{Na}]^{+}\right)$: 369.1588 , found: 369.1579 .

3-hydroxy-1',3,9-trimethyl-1,2,3,9-tetrahydrospiro[carbazole-4,3'-indolin]-2'-one, 
(syn-3b):

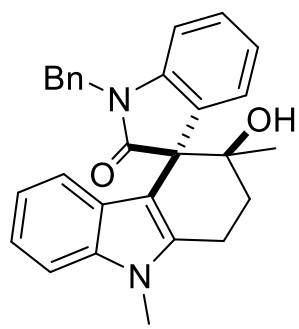

White solid, $92 \%$ yield, ${ }^{1} \mathrm{H}$ NMR (400 MHz, CDCl3) $\delta 7.50$ (d, $J=$ $7.4 \mathrm{~Hz}, 2 \mathrm{H}), 7.41-7.29(\mathrm{~m}, 3 \mathrm{H}), 7.20$ (d, $J=8.2 \mathrm{~Hz}, 2 \mathrm{H}), 7.08-$ $7.00(\mathrm{~m}, 2 \mathrm{H}), 6.94(\mathrm{dd}, J=21.8,7.8 \mathrm{~Hz}, 2 \mathrm{H}), 6.72(\mathrm{t}, J=7.7 \mathrm{~Hz}$, $1 \mathrm{H}), 6.42(\mathrm{~d}, J=8.0 \mathrm{~Hz}, 1 \mathrm{H}), 5.24(\mathrm{~d}, J=15.3 \mathrm{~Hz}, 1 \mathrm{H}), 5.02(\mathrm{~d}, J$ $=15.2 \mathrm{~Hz}, 1 \mathrm{H}), 4.31(\mathrm{~s}, 1 \mathrm{H}), 3.68(\mathrm{~s}, 2 \mathrm{H}), 3.29-3.16(\mathrm{~m}, 1 \mathrm{H}), 2.89$ $(\mathrm{d}, J=17.0 \mathrm{~Hz}, 1 \mathrm{H}), 2.50(\mathrm{~d}, J=14.1 \mathrm{~Hz}, 1 \mathrm{H}), 2.33-2.21(\mathrm{~m}, 2 \mathrm{H}), 1.10(\mathrm{~s}, 3 \mathrm{H}) .{ }^{13} \mathrm{C}$ NMR (100 MHz, CDCl3) $\delta 179.7,142.7,137.3,136.7,135.8,132.4,128.9,128.1$, 128.1, 127.9, 125.4, 125.2, 122.9, 120.8, 119.0, 117.6, 109.2, 108.8, 106.8, 100.0, 77.3, 77.0, 76.7, 73.3, 56.2, 44.4, 32.5, 29.3, 25.3, 19.4. HRMS(ESI) Calcd. for $\mathrm{C}_{28} \mathrm{H}_{27} \mathrm{~N}_{2} \mathrm{O}_{2}$ $(\mathrm{M}+\mathrm{H})^{+}$423.2079, found: 423.2073 .

3-hydroxy-1',3,9-trimethyl-1,2,3,9-tetrahydrospiro[carbazole-4,3'-indolin]-2'-one, (syn-3c):

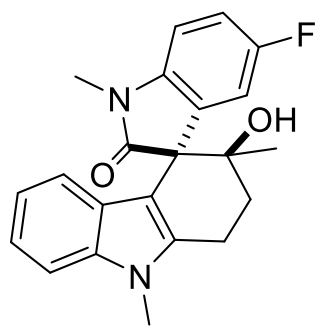

White solid, $83 \%$ yield, ${ }^{1} \mathrm{H}$ NMR (400 MHz, Chloroform-d) $\delta$ $7.23(\mathrm{~d}, J=8.1 \mathrm{~Hz}, 1 \mathrm{H}), 7.07(\mathrm{t}, J=8.0 \mathrm{~Hz}, 1 \mathrm{H}), 6.97(\mathrm{t}, 1 \mathrm{H}), 6.95$ $-6.89(\mathrm{~m}, 1 \mathrm{H}), 6.86(\mathrm{t}, J=7.7 \mathrm{~Hz}, 1 \mathrm{H}), 6.80(\mathrm{~d}, J=8.3 \mathrm{~Hz}, 1 \mathrm{H})$,

$6.57(\mathrm{~d}, J=8.0 \mathrm{~Hz}, 1 \mathrm{H}), 4.58(\mathrm{~s}, 1 \mathrm{H}), 3.69(\mathrm{~s}, 3 \mathrm{H}), 3.46(\mathrm{~s}, 3 \mathrm{H})$, $3.22(\mathrm{dt}, J=16.7,7.8 \mathrm{~Hz}, 1 \mathrm{H}), 2.89(\mathrm{dd}, 1 \mathrm{H}), 2.36(\mathrm{dd}, J=14.4,5.9 \mathrm{~Hz}, 1 \mathrm{H}), 2.31-$ $2.20(\mathrm{~m}, 1 \mathrm{H}), 1.04(\mathrm{~s}, 3 \mathrm{H}) .{ }^{13} \mathrm{C}$ NMR (100 MHz, Chloroform-d) $\delta 179.3,141.8,137.3$, 136.8, 134.5, $128.4(\mathrm{~d}, J=43.0 \mathrm{~Hz}), 125.2(\mathrm{~d}, J=42.1 \mathrm{~Hz}), 121.0,119.3,116.9,109.2$, 109.0, 72.9, 56.2, 32.4, 29.3, 26.7, 25.1, 19.1. ${ }^{19} \mathrm{~F}$ NMR (400 MHz, CDCl3) $\delta-119.73$. HRMS(ESI) Calcd. for $\mathrm{C}_{22} \mathrm{H}_{21} \mathrm{~N}_{2} \mathrm{O}_{2} \mathrm{NaF}(\mathrm{M}+\mathrm{Na})^{+}$387.1504, found: 387.1485 .

5'-chloro-3-hydroxy-1',3,9-trimethyl-1,2,3,9-tetrahydrospiro[carbazole-4,3'indolin]-2'-one (syn-3d): 


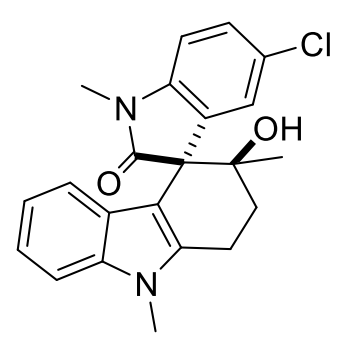

White solid, $92 \%$ yield, ${ }^{1} \mathrm{H}$ NMR (400 MHz, Chloroform-d) $\delta 7.23$ $(\mathrm{d}, J=7.7 \mathrm{~Hz}, 2 \mathrm{H}), 7.07(\mathrm{t}, J=7.8 \mathrm{~Hz}, 1 \mathrm{H}), 7.01(\mathrm{~s}, 1 \mathrm{H}), 6.92(\mathrm{~d}$, $J=8.4 \mathrm{~Hz}, 1 \mathrm{H}), 6.85(\mathrm{t}, J=7.6 \mathrm{~Hz}, 1 \mathrm{H}), 6.57(\mathrm{~d}, J=7.9 \mathrm{~Hz}, 1 \mathrm{H})$, $4.51(\mathrm{~s}, 1 \mathrm{H}), 3.69$ (s, 3H), 3.46 (s, 3H), $3.22(\mathrm{dt}, J=16.9,7.6 \mathrm{~Hz}$, $1 \mathrm{H}), 2.90(\mathrm{dd}, J=17.8,4.9 \mathrm{~Hz}, 1 \mathrm{H}), 2.41-2.31(\mathrm{~m}, 1 \mathrm{H}), 2.31-$ 2.21 (m, 1H), 1.04 (s, 3H). ${ }^{13} \mathrm{C}$ NMR (100 MHz, Chloroform-d) $\delta 179.3,141.8,137.3$, 136.8, 134.5, 128.6, 128.1, 125.4, 125.0, 121.0, 119.3, 116.9, 109.2, 109.0, 72.9, 56.2, 32.4, 29.3, 26.7, 25.1, 19.1. HRMS(ESI) Calcd. for $\mathrm{C}_{22} \mathrm{H}_{21} \mathrm{~N}_{2} \mathrm{O}_{2} \mathrm{NaCl}(\mathrm{M}+\mathrm{Na}){ }^{+}$ 403.1187, found: 403.1189 .

\section{3-hydroxy-5'-methoxy-1',3,9-trimethyl-1,2,3,9-tetrahydrospiro[carbazole-4,3'-} indolin]-2'-one (syn-3e):

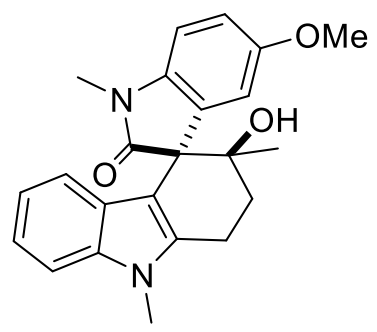

White solid, 99\% yield, ${ }^{1} \mathrm{H}$ NMR (400 MHz, Chloroform- $d$ ) $\delta$ $7.21(\mathrm{~s}, 1 \mathrm{H}), 7.05(\mathrm{t}, J=7.8 \mathrm{~Hz}, 1 \mathrm{H}), 6.90(\mathrm{~d}, J=8.6 \mathrm{~Hz}, 1 \mathrm{H})$, $6.84(\mathrm{t}, J=7.7 \mathrm{~Hz}, 1 \mathrm{H}), 6.78(\mathrm{~d}, J=8.7 \mathrm{~Hz}, 1 \mathrm{H}), 6.67-6.59$ $(\mathrm{m}, 2 \mathrm{H}), 4.80(\mathrm{~s}, 1 \mathrm{H}), 3.68(\mathrm{~s}, 6 \mathrm{H}), 3.46(\mathrm{~s}, 3 \mathrm{H}), 3.22(\mathrm{dt}, J=$ $16.9,8.1 \mathrm{~Hz}, 1 \mathrm{H}), 2.88(\mathrm{~d}, J=17.1 \mathrm{~Hz}, 1 \mathrm{H}), 2.36-2.25$ (m, 2H), 1.02 (s, 3H). ${ }^{13} \mathrm{C}$ NMR (100 MHz, Chloroform- $d$ ) $\delta$ 179.4, 156.3, 137.3, 136.8, 136.6, 134.3, 125.2, 120.8, 119.2, 117.1, 113.0, 111.8, 108.9, 108.5, 106.6, 72.8, 56.1, 55.7, 32.3, 29.3, 26.6, 25.1, 19.1. HRMS(ESI) Calcd. for $\mathrm{C}_{23} \mathrm{H}_{24} \mathrm{~N}_{2} \mathrm{O}_{3} \mathrm{Na}(\mathrm{M}+\mathrm{Na})^{+}$ 399.1697, found: 399.1685 .

\section{3-hydroxy-1',9-dimethyl-3-phenyl-1,2,3,9-tetrahydrospiro[carbazole-4,3'-} indolin]-2'-one (syn-3f):

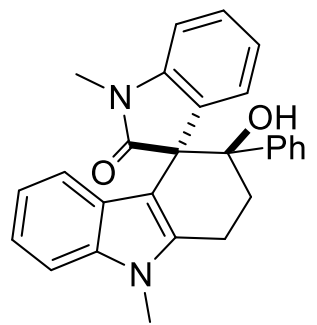

White solid, 95\% yield, ${ }^{1} \mathrm{H}$ NMR (400 MHz, Chloroform- $d$ ) $\delta 7.27$ $(\mathrm{s}, 1 \mathrm{H}), 7.16(\mathrm{~d}, J=7.6 \mathrm{~Hz}, 1 \mathrm{H}), 7.13-6.95(\mathrm{~m}, 7 \mathrm{H}), 6.92(\mathrm{t}, J=$ $7.8 \mathrm{~Hz}, 1 \mathrm{H}), 6.85(\mathrm{t}, J=7.7 \mathrm{~Hz}, 1 \mathrm{H}), 6.64(\mathrm{~d}, J=7.9 \mathrm{~Hz}, 1 \mathrm{H}), 6.52$ (d, $J=7.8 \mathrm{~Hz}, 1 \mathrm{H}), 5.94(\mathrm{~s}, 1 \mathrm{H}), 3.75(\mathrm{~s}, 3 \mathrm{H}), 3.39$ (td, $J=12.5$, $5.8 \mathrm{~Hz}, 1 \mathrm{H}), 3.13-2.99(\mathrm{~m}, 5 \mathrm{H}), 2.39(\mathrm{dd}, J=12.8,6.1 \mathrm{~Hz}, 1 \mathrm{H})$. ${ }^{13} \mathrm{C}$ NMR $\left(100 \mathrm{MHz}, \mathrm{CDCl}_{3}\right) \delta 179.2,142.7,142.4,137.2,137.0,132.1,128.0,126.8$, 
126.7, 125.7, 125.4, 125.0, 122.8, 120.9, 119.3, 117.1, 109.0, 107.9, 106.1, 77.4, 77.2, 77.0, 76.7, 76.3, 57.1, 30.8, 29.4, 26.1, 19.4. HRMS(ESI) Calcd. for $\mathrm{C}_{27} \mathrm{H}_{24} \mathrm{~N}_{2} \mathrm{O}_{2} \mathrm{Na}$ $(\mathrm{M}+\mathrm{Na})^{+} 431.1760$, found: 431.1735.

\section{9-benzyl-3-hydroxy-1'-methyl-3-phenyl-1,2,3,9-tetrahydrospiro[carbazole-4,3'- indolin]-2'-one (syn-3g):}

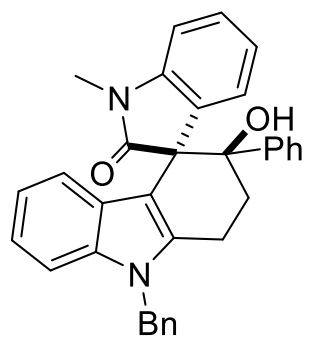

White solid, 87\% yield, ${ }^{1} \mathrm{H}$ NMR (400 MHz, Chloroform-d) $\delta 7.45$ (d, $J=7.5 \mathrm{~Hz}, 1 \mathrm{H}), 7.32(\mathrm{q}, J=8.5,7.9 \mathrm{~Hz}, 4 \mathrm{H}), 7.20(\mathrm{~d}, J=8.3$ $\mathrm{Hz}, 1 \mathrm{H}), 7.15-7.03(\mathrm{~m}, 8 \mathrm{H}), 7.00(\mathrm{t}, J=7.7 \mathrm{~Hz}, 1 \mathrm{H}), 6.72(\mathrm{t}, J=$ $7.6 \mathrm{~Hz}, 1 \mathrm{H}), 6.60(\mathrm{~d}, J=7.8 \mathrm{~Hz}, 1 \mathrm{H}), 6.10(\mathrm{~d}, J=8.0 \mathrm{~Hz}, 1 \mathrm{H}), 5.39$ $(\mathrm{dd}, J=3.8 \mathrm{~Hz}, 2 \mathrm{H}), 3.87(\mathrm{q}, J=9.4 \mathrm{~Hz}, 1 \mathrm{H}), 3.17-3.08(\mathrm{~m}, 2 \mathrm{H})$, $2.76(\mathrm{~s}, 3 \mathrm{H}), 2.62(\mathrm{~s}, 1 \mathrm{H}), 2.13(\mathrm{~d}, J=13.4 \mathrm{~Hz}, 1 \mathrm{H}) .{ }^{13} \mathrm{C} \mathrm{NMR}(100 \mathrm{MHz}, \mathrm{CDCl} 3) \delta$ $176.4,144.1,141.2$, 137.5, 137.3, 136.9, 128.9, 128.5, 127.4, 127.4, 127.3, 126.9, 126.2, $125.9,122.1,121.2,119.3,118.6,109.3,107.3,106.7,77.3,77.2,77.0,76.7,75.9,59.2$, $46.7,29.5,25.9,18.8,0.0$.

HRMS (ESI) Calcd. for $\mathrm{C}_{33} \mathrm{H}_{29} \mathrm{~N}_{2} \mathrm{O}_{2}(\mathrm{M}+\mathrm{H})^{+} 485.2250$, found: 485.2229.

\section{3-butyl-3-hydroxy-1',9-dimethyl-1,2,3,9-tetrahydrospiro[carbazole-4,3'-indolin]-} 2'-one (syn-3h):

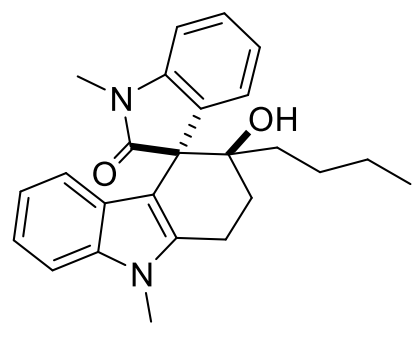

White solid, 91\% yield, ${ }^{1} \mathrm{H}$ NMR (400 MHz, Chloroform-d) $\delta 7.30-7.26(\mathrm{~m}, 1 \mathrm{H}), 7.20(\mathrm{~d}, J=8.2 \mathrm{~Hz}, 1 \mathrm{H}), 7.06-6.92$ $(\mathrm{m}, 4 \mathrm{H}), 6.82(\mathrm{t}, J=7.7 \mathrm{~Hz}, 1 \mathrm{H}), 6.58(\mathrm{~d}, J=8.0 \mathrm{~Hz}, 1 \mathrm{H})$, $4.54(\mathrm{~s}, 1 \mathrm{H}), 3.68(\mathrm{~s}, 3 \mathrm{H}), 3.46(\mathrm{~s}, 3 \mathrm{H}), 3.18(\mathrm{dt}, J=16.7,8.0$ $\mathrm{Hz}, 1 \mathrm{H}), 2.87(\mathrm{dd}, J=17.1,5.6 \mathrm{~Hz}, 1 \mathrm{H}), 2.49(\mathrm{dd}, J=14.7$, $6.0 \mathrm{~Hz}, 1 \mathrm{H}), 2.23-2.13(\mathrm{~m}, 1 \mathrm{H}), 1.47-1.01(\mathrm{~m}, 7 \mathrm{H}), 0.79(\mathrm{t}, J=7.4 \mathrm{~Hz}, 3 \mathrm{H}) .{ }^{13} \mathrm{C}$ NMR (100 MHz, CDCl3) $\delta 180.1,143.1,137.2,136.8,132.7,128.0,125.2,123.2$, 120.8, 119.2, 117.0, 108.9, 108.4, 106.8, 77.4, 77.2, 77.0, 76.7, 75.0, 56.2, 36.8, 29.3, 29.0, 26.5, 25.2, 23.3, 19.0, 14.1. HRMS (ESI) Calcd.for $\mathrm{C}_{33} \mathrm{H}_{29} \mathrm{~N}_{2} \mathrm{O}_{2}(\mathrm{M}+\mathrm{H})^{+} 485.2250$, found: 485.2229 . 
1'-(4-bromobenzyl)-3-hydroxy-3,9-dimethyl-1,2,3,9-tetrahydrospiro[carbazole4,3'-indolin]-2'-one (syn-3i):

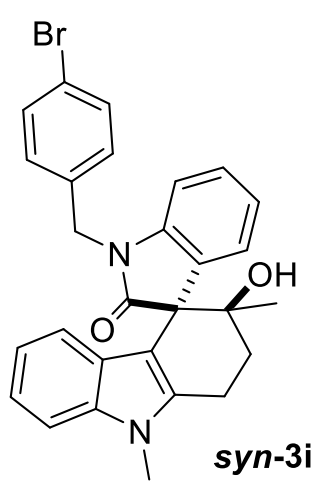

White solid, 90\% yield, ${ }^{1} \mathrm{H}$ NMR (400 MHz, Chloroform-d) $\delta 7.53$ $-7.46(\mathrm{~m}, 2 \mathrm{H}), 7.37$ (d, $J=7.9 \mathrm{~Hz}, 2 \mathrm{H}), 7.24-7.18(\mathrm{~m}, 2 \mathrm{H}), 7.08$ $-7.02(\mathrm{~m}, 2 \mathrm{H}), 6.97-6.89(\mathrm{~m}, 2 \mathrm{H}), 6.72(\mathrm{t}, J=7.7 \mathrm{~Hz}, 1 \mathrm{H}), 6.32$ $(\mathrm{d}, J=8.0 \mathrm{~Hz}, 1 \mathrm{H}), 5.15(\mathrm{~d}, J=15.4 \mathrm{~Hz}, 1 \mathrm{H}), 4.97(\mathrm{~d}, J=15.3 \mathrm{~Hz}$, 1H), 4.06 (s, 1H), 3.68 (s, 3H), $3.21(\mathrm{dt}, J=16.0,7.3 \mathrm{~Hz}, 1 \mathrm{H}), 2.94$ $-2.85(\mathrm{~m}, 1 \mathrm{H}), 2.59-2.50(\mathrm{~m}, 1 \mathrm{H}), 2.24(\mathrm{dt}, J=14.1,7.1 \mathrm{~Hz}, 1 \mathrm{H})$, 1.11 (s, 3H). ${ }^{13} \mathrm{C}$ NMR (100 MHz, Chloroform-d) $\delta 179.7,142.7$, 136.7, 129.6, 128.0, 127.9, 125.3, 122.9, 120.8, 119.0, 117.6, 114.2, 109.2, 108.8, 73.2, 56.1, 55.3, 43.8, 32.5, 29.3, 25.2, 19.3. HRMS(ESI) Calcd. for $\mathrm{C}_{28} \mathrm{H}_{25} \mathrm{~N}_{2} \mathrm{O}_{2} \mathrm{NaBr}$ $(\mathrm{M}+\mathrm{Na})^{+}$523.1001, found: 523.0997.

3-hydroxy-1'-(4-methoxybenzyl)-3,9-dimethyl-1,2,3,9-tetrahydrospiro[carbazole4,3'-indolin]-2'-one (syn-3j):

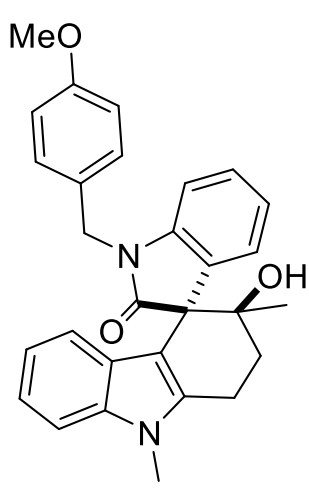

White solid, 99\% yield, ${ }^{1} \mathrm{H}$ NMR (400 MHz, Chloroform-d) $\delta 7.44$ $(\mathrm{d}, J=8.1 \mathrm{~Hz}, 2 \mathrm{H}), 7.23-7.17(\mathrm{~m}, 2 \mathrm{H}), 7.07-6.98(\mathrm{~m}, 3 \mathrm{H}), 6.94$ $-6.87(\mathrm{~m}, 3 \mathrm{H}), 6.71(\mathrm{t}, J=7.7 \mathrm{~Hz}, 1 \mathrm{H}), 6.40(\mathrm{~d}, J=8.0 \mathrm{~Hz}, 1 \mathrm{H})$, $5.15(\mathrm{~d}, J=15.1 \mathrm{~Hz}, 1 \mathrm{H}), 4.98(\mathrm{~d}, J=15.1 \mathrm{~Hz}, 1 \mathrm{H}), 4.41(\mathrm{~s}, 1 \mathrm{H})$, $3.81(\mathrm{~s}, 3 \mathrm{H}), 3.68(\mathrm{~s}, 3 \mathrm{H}), 3.22(\mathrm{dt}, J=15.5,7.5 \mathrm{~Hz}, 1 \mathrm{H}), 2.93-$ $2.84(\mathrm{~m}, 1 \mathrm{H}), 2.46(\mathrm{~d}, J=13.9 \mathrm{~Hz}, 1 \mathrm{H}), 2.33-2.21(\mathrm{~m}, 1 \mathrm{H}), 1.07$ (s, 3H). ${ }^{13} \mathrm{C}$ NMR (100 MHz, CDCl3) $\delta 179.7,159.3,142.7,136.7$, 129.6, 128.0, 127.9, 125.3, 125.2, 122.9, 120.8, 119.0, 117.6, 114.2, 109.2, 108.8, 77.3, 77.0, 76.7, 73.2, 56.1, 55.3, 43.8, 32.5, 29.3, 25.2, 19.3. HRMS(ESI) Calcd. for: $\mathrm{C}_{29} \mathrm{H}_{29} \mathrm{~N}_{2} \mathrm{O}_{3}(\mathrm{M}+\mathrm{H})^{+} 453.2175$, found: 453.2178 .

1'-benzyl-3-hydroxy-5'-methoxy-3,9-dimethyl-1,2,3,9-tetrahydrospiro[carbazole4,3'-indolin]-2' -one (syn-3k): 


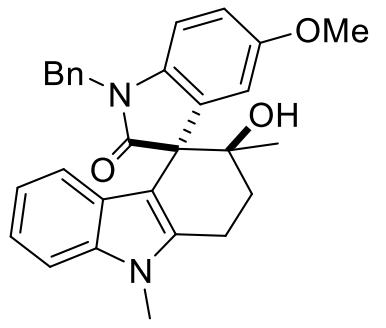

White solid, $89 \%$ yield, ${ }^{1} \mathrm{H}$ NMR (400 MHz, Chloroform-d) $\delta$ $7.50(\mathrm{~d}, J=7.4 \mathrm{~Hz}, 2 \mathrm{H}), 7.42-7.29(\mathrm{~m}, 3 \mathrm{H}), 7.21(\mathrm{~d}, J=8.1$ $\mathrm{Hz}, 1 \mathrm{H}), 7.04$ (t, $J=7.8 \mathrm{~Hz}, 1 \mathrm{H}), 6.86(\mathrm{~d}, J=8.6 \mathrm{~Hz}, 1 \mathrm{H})$, $6.77-6.67(\mathrm{~m}, 2 \mathrm{H}), 6.64(\mathrm{~s}, 1 \mathrm{H}), 6.49(\mathrm{~d}, J=8.0 \mathrm{~Hz}, 1 \mathrm{H})$, $5.23(\mathrm{~d}, J=15.2 \mathrm{~Hz}, 1 \mathrm{H}), 5.00(\mathrm{~d}, J=15.3 \mathrm{~Hz}, 1 \mathrm{H}), 4.59$ (s, $1 \mathrm{H}), 3.68(\mathrm{~d}, J=2.2 \mathrm{~Hz}, 3 \mathrm{H}), 3.64(\mathrm{~d}, J=2.2 \mathrm{~Hz}, 3 \mathrm{H}), 3.22(\mathrm{dt}, \mathrm{J}=16.6,8.0 \mathrm{~Hz}, 1 \mathrm{H})$, $2.93-2.83(\mathrm{~m}, 1 \mathrm{H}), 2.46-2.36(\mathrm{~m}, 1 \mathrm{H}), 2.35-2.22(\mathrm{~m}, 1 \mathrm{H}), 1.09(\mathrm{~s}, 3 \mathrm{H}) .{ }^{13} \mathrm{C}$ NMR $(100 \mathrm{MHz}, \mathrm{CDCl} 3) \delta 179.4,156.1,137.3,136.7,136.1,135.8,134.1,128.9,128.2$, 127.9, 125.2, 120.8, 119.0, 117.5, 113.2, 111.8, 109.5, 108.8, 106.8, 77.4, 77.0, 76.7, 73.1, 56.3, 55.6, 44.5, 32.4, 29.3, 25.3, 19.2. HRMS(ESI) Calcd. for: $\mathrm{C}_{29} \mathrm{H}_{29} \mathrm{~N}_{2} \mathrm{O}_{3}$ $(\mathrm{M}+\mathrm{H})^{+}$453.2175, found: 453.2178 .

\section{1'-benzyl-3-hydroxy-3,5',9-trimethyl-1,2,3,9-tetrahydrospiro[carbazole-4,3'-} indolin]-2'-one (syn-31):

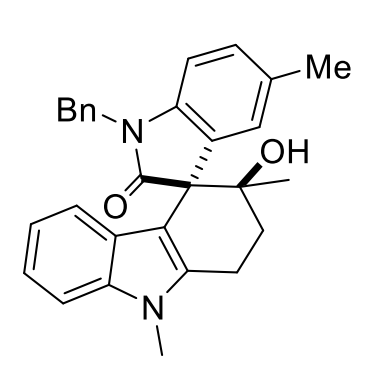

White solid, $80 \%$ yield, ${ }^{1} \mathrm{H}$ NMR (400 MHz, Chloroform- $d$ ) $\delta$ $7.50(\mathrm{~d}, J=7.3 \mathrm{~Hz}, 2 \mathrm{H}), 7.40-7.29(\mathrm{~m}, 3 \mathrm{H}), 7.21(\mathrm{~d}, J=8.3 \mathrm{~Hz}$, 1H), 7.04 (t, $J=7.4 \mathrm{~Hz}, 2 \mathrm{H}), 6.98(\mathrm{~d}, J=8.1 \mathrm{~Hz}, 1 \mathrm{H}), 6.85$ (d, $J$ $=9.0 \mathrm{~Hz}, 2 \mathrm{H}), 6.73(\mathrm{t}, J=7.3 \mathrm{~Hz}, 2 \mathrm{H}), 6.47(\mathrm{~d}, J=7.9 \mathrm{~Hz}, 1 \mathrm{H})$, $5.23(\mathrm{~d}, J=15.2 \mathrm{~Hz}, 1 \mathrm{H}), 5.00(\mathrm{~d}, J=15.3 \mathrm{~Hz}, 1 \mathrm{H}), 4.54(\mathrm{~s}, 1 \mathrm{H})$, $3.69(\mathrm{~s}, 3 \mathrm{H}), 3.23(\mathrm{dt}, J=16.5,8.0 \mathrm{~Hz}, 1 \mathrm{H}), 2.94-2.85(\mathrm{~m}, 1 \mathrm{H}), 2.46-2.37(\mathrm{~m}, 1 \mathrm{H})$, $2.35-2.26(\mathrm{~m}, 1 \mathrm{H}), 2.17(\mathrm{~s}, 3 \mathrm{H}), 1.08(\mathrm{~s}, 3 \mathrm{H}) .{ }^{13} \mathrm{C} \mathrm{NMR}\left(100 \mathrm{MHz}, \mathrm{CDCl}_{3}\right) \delta 179.7$, 140.2, 137.3, 136.7, 135.9, 132.6, 132.4, 128.8, 128.4, 128.2, 127.9, 126.0, 125.2, 120.7, 119.0, 117.6, 109.0, 108.8, 106.9, 77.4, 77.0, 76.7, 73.2, 56.0, 44.4, 32.5, 29.3, 25.3, 21.2, 19.2. HRMS(ESI) Calcd. for $\mathrm{C}_{29} \mathrm{H}_{29} \mathrm{~N}_{2} \mathrm{O}_{2}(\mathrm{M}+\mathrm{H})^{+} 437.2201$, found: 437.2229.

1'-benzyl-5'-chloro-3-hydroxy-3,9-dimethyl-1,2,3,9-tetrahydrospiro[carbazole4,3'-indolin]-2'-one (syn-3m): 


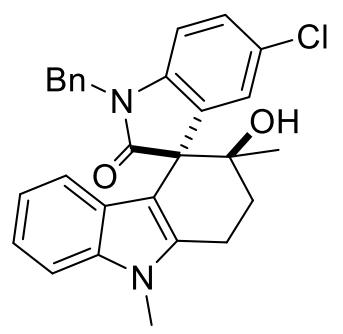

White solid, $95 \%$ yield, ${ }^{1} \mathrm{H}$ NMR (400 MHz, Chloroform- $d$ ) $\delta 7.47$ (d, $J=7.4 \mathrm{~Hz}, 2 \mathrm{H}), 7.41-7.32(\mathrm{~m}, 3 \mathrm{H}), 7.23(\mathrm{~d}, J=8.1 \mathrm{~Hz}, 1 \mathrm{H})$, $7.16(\mathrm{~d}, J=8.5 \mathrm{~Hz}, 1 \mathrm{H}), 7.06(\mathrm{t}, J=7.8 \mathrm{~Hz}, 1 \mathrm{H}), 7.01(\mathrm{~s}, 1 \mathrm{H})$, $6.88(\mathrm{~d}, J=8.3 \mathrm{~Hz}, 1 \mathrm{H}), 6.75(\mathrm{t}, J=7.6 \mathrm{~Hz}, 1 \mathrm{H}), 6.42(\mathrm{~d}, J=7.9$ $\mathrm{Hz}, 1 \mathrm{H}), 5.22(\mathrm{~d}, J=15.3 \mathrm{~Hz}, 1 \mathrm{H}), 5.00(\mathrm{~d}, J=15.3 \mathrm{~Hz}, 1 \mathrm{H}), 4.26$ (s, 1H), 3.69 (s, 3H), $3.22(\mathrm{dt}, J=16.3,7.8 \mathrm{~Hz}, 1 \mathrm{H}), 2.90(\mathrm{~d}, J=16.8 \mathrm{~Hz}, 1 \mathrm{H}), 2.49$ (d, $J=13.7 \mathrm{~Hz}, 1 \mathrm{H}), 2.23(\mathrm{dt}, J=14.0,7.6 \mathrm{~Hz}, 1 \mathrm{H}), 1.11(\mathrm{~s}, 3 \mathrm{H}) .{ }^{13} \mathrm{C} \mathrm{NMR}(100 \mathrm{MHz}$, $\left.\mathrm{CDCl}_{3}\right) \delta 179.3,141.3,137.3,136.8,135.3,134.2,129.0,128.4,128.1,125.7,125.0$, 125.0, 121.0, 119.2, 117.4, 110.2, 109.0, 106.1, 77.3, 77.2, 77.0, 76.8, 76.7, 73.3, 56.4, 44.5, 32.6, 29.3, 25.2, 19.3. HRMS(ESI) Calcd. for $\mathrm{C}_{28} \mathrm{H}_{26} \mathrm{~N}_{2} \mathrm{O}_{2} \mathrm{Cl}(\mathrm{M}+\mathrm{H})^{+} 457.1655$, found: 457.1683 .

\section{1'-benzyl-5'-bromo-3-hydroxy-3,9-dimethyl-1,2,3,9-tetrahydrospiro[carbazole-} 4,3'-indolin]-2'-one (syn-3n):

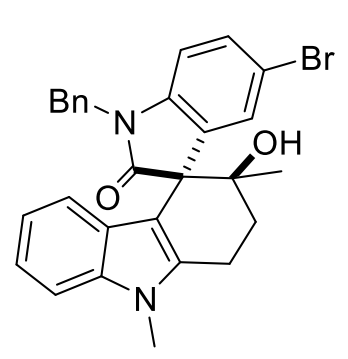

White solid, 95\% yield, ${ }^{1} \mathrm{H}$ NMR (400 MHz, Chloroform- $d$ ) $\delta$ $7.37-7.28(\mathrm{~m}, 5 \mathrm{H}), 7.22(\mathrm{~s}, 1 \mathrm{H}), 7.07$ (q, $J=9.1,8.1 \mathrm{~Hz}, 3 \mathrm{H})$, $6.80(\mathrm{~d}, J=7.3 \mathrm{~Hz}, 1 \mathrm{H}), 6.69(\mathrm{t}, J=7.6 \mathrm{~Hz}, 1 \mathrm{H}), 6.25(\mathrm{~d}, J=8.1$ $\mathrm{Hz}, 1 \mathrm{H}), 5.13(\mathrm{~d}, J=15.8 \mathrm{~Hz}, 1 \mathrm{H}), 4.85(\mathrm{~s}, 1 \mathrm{H}), 4.79$ (d, $J=15.5$ $\mathrm{Hz}, 1 \mathrm{H}), 3.75(\mathrm{~s}, 3 \mathrm{H}), 3.38-3.24(\mathrm{~m}, 2 \mathrm{H}), 3.14(\mathrm{dt}, J=15.5,7.3$ $\mathrm{Hz}, 1 \mathrm{H}), 3.05-2.96(\mathrm{~m}, 1 \mathrm{H}), 2.23$ (s, 3H). ${ }^{13} \mathrm{C} \mathrm{NMR}\left(100 \mathrm{MHz}, \mathrm{CDCl}_{3}\right) \delta 207.5,175.8$, 145.0, 139.2, 136.9, 135.5, 129.6, 128.8, 128.4, 127.8, 127.6, 126.3, 121.0, 120.2, 119.2, 118.0, 109.0, 107.9, 104.0, 77.3, 77.2, 77.0, 76.7, 45.9, 44.3, 44.0, 30.2, 29.9, 18.6, 0.0. HRMS(ESI) Calcd. for $\mathrm{C}_{28} \mathrm{H}_{25} \mathrm{BrN}_{2} \mathrm{O}_{2} \mathrm{Na}(\mathrm{M}+\mathrm{Na})^{+} 523.1107$, found: 523.1099. 


\section{3-hydroxy-1',9-dimethyl-3-(4-(trifluoromethyl)phenyl)-1,2,3,9-tetrahydrospiro}

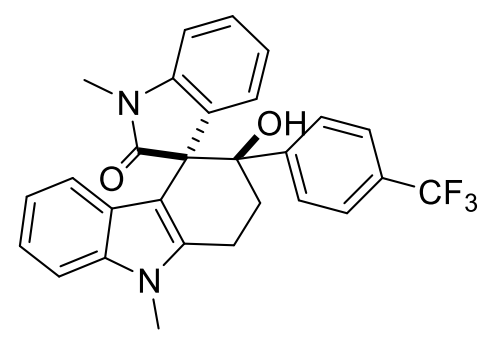

[carbazole-4,3'-indolin]-2'-one (syn-3o):

White solid, 99\% yield, ${ }^{1} \mathrm{H}$ NMR (400 MHz,

Chloroform-d) $\delta 7.28(\mathrm{~s}, 1 \mathrm{H}), 7.20-7.06(\mathrm{~m}, 5 \mathrm{H}), 6.95$ $(\mathrm{t}, J=7.7 \mathrm{~Hz}, 1 \mathrm{H}), 6.86(\mathrm{t}, J=7.6 \mathrm{~Hz}, 1 \mathrm{H}), 6.62(\mathrm{~d}, J=$ $8.0 \mathrm{~Hz}, 1 \mathrm{H}), 6.56(\mathrm{~d}, J=7.9 \mathrm{~Hz}, 1 \mathrm{H}), 6.01(\mathrm{~s}, 1 \mathrm{H}), 3.76$ (s, 3H), 3.39 (p, $J=9.1 \mathrm{~Hz}, 1 \mathrm{H}), 3.12-2.99(\mathrm{~m}, 5 \mathrm{H}), 2.37$ (dd, $J=14.3,5.9 \mathrm{~Hz}, 1 \mathrm{H})$. ${ }^{13} \mathrm{C} \mathrm{NMR}\left(100 \mathrm{MHz}, \mathrm{CDCl}_{3}\right) \delta 178.9,146.6,142.5,137.2,136.8,131.5, \delta 129.6-128.5$ (m), 128.5, 126.3, 125.2, 125.0, 123.6, 123.5, 123.0, 121.1, 119.4, 117.0, 109.0, 108.2, $105.8,77.3,77.0,76.7,76.2,56.9,30.8,29.4,26.1,19.3 .{ }^{19} \mathrm{~F}$ NMR $(376 \mathrm{MHz}, \mathrm{CDCl} 3)$ $\delta$-62.49. HRMS(ESI) Calcd. for $\mathrm{C}_{28} \mathrm{H}_{24} \mathrm{~N}_{2} \mathrm{O}_{2} \mathrm{~F}_{3}(\mathrm{M}+\mathrm{Na})^{+} 477.1788$, found: 477.1790.

Methyl 4-(4-bromophenyl)-3-hydroxy-9-methyl-3-phenyl-2,3,4,9-tetrahydro- $1 \mathrm{H}$ carbazole-4-carboxylate (syn-5a):

White solid, 73\% yield, ${ }^{1} \mathrm{H}$ NMR $(400 \mathrm{MHz}$, Chloroform- $d) \delta 7.35$
$(\mathrm{~d}, J=8.2 \mathrm{~Hz}, 1 \mathrm{H}), 7.20-7.11(\mathrm{~m}, 4 \mathrm{H}), 7.10-7.02(\mathrm{~m}, 2 \mathrm{H}), 7.02$
$(\mathrm{~d}, J=8.1 \mathrm{~Hz}, 2 \mathrm{H}), 5.72(\mathrm{~s}, 1 \mathrm{H}), 3.77(\mathrm{~s}, 3 \mathrm{H}), 3.73(\mathrm{~s}, 3 \mathrm{H}), 3.29-$
$(\mathrm{m}, 1 \mathrm{H}),{ }^{13} \mathrm{C} \mathrm{NMR}\left(100 \mathrm{MHz}, \mathrm{CDCl}_{3}\right) \delta 176.1,144.0,137.7,137.7$, $136.56,132.6,132.5,127.3,127.1,126.9,126.2$, 126.1, 121.1, 119.8, 119.4, 108.9, 108.2, 77.8, 61.5, 52.5, 32.2, 29.4, 20.0, 0.0. HRMS(ESI) Calcd. for $\mathrm{C}_{27} \mathrm{H}_{24} \mathrm{BrNO}_{3} \mathrm{Na}$ $(\mathrm{M}+\mathrm{Na})^{+}$512.1931, found: 512.1926 .

Methyl 4-(4-chlorophenyl)-3-hydroxy-9-methyl-3-phenyl-2,3,4,9-tetrahydro-1 Hcarbazole-4-carboxylate (syn-5b): 
White solid, 50\% yield, ${ }^{1} \mathrm{H}$ NMR (400 MHz, Chloroform- $d$ ) $\delta 7.35$<smiles></smiles>
$(\mathrm{d}, J=8.2 \mathrm{~Hz}, 1 \mathrm{H}), 7.21-7.10(\mathrm{~m}, 4 \mathrm{H}), 7.10-7.02(\mathrm{~m}, 2 \mathrm{H}), 7.02$ - $6.98(\mathrm{~m}, 1 \mathrm{H}), 6.96-6.91(\mathrm{~m}, 1 \mathrm{H}), 6.88(\mathrm{~d}, J=8.2 \mathrm{~Hz}, 2 \mathrm{H}), 6.55$ $(\mathrm{d}, J=7.7 \mathrm{~Hz}, 2 \mathrm{H}), 5.72(\mathrm{~s}, 1 \mathrm{H}), 3.78(\mathrm{~s}, 3 \mathrm{H}), 3.73(\mathrm{~s}, 3 \mathrm{H}), 3.28-$ $3.18(\mathrm{~m}, 1 \mathrm{H}), 2.98-2.88(\mathrm{~m}, 1 \mathrm{H}), 2.52-2.40$ (m, 1H), $2.37-2.25$

(m, 1H). ${ }^{13} \mathrm{C}$ NMR (100 MHz, $\left.\mathrm{CDCl}_{3}\right) \delta 176.1,144.0,137.8,137.7$, 136.6, 132.6, 132.5, 127.3, 127.2, 126.9, 126.2, 126.1, 121.1, 119.8, 119.4, 108.9, 108.2, 77.8, 61.5, 52.5, 32.2, 29.7, 29.5, 20.0, 0.0. HRMS(ESI) Calcd. for $\mathrm{C}_{27} \mathrm{H}_{24} \mathrm{ClNO}_{3}$ $(\mathrm{M}+\mathrm{Na})^{+}$468.1365, found: 468.1342 .

Methyl 3-hydroxy-4-(4-methoxyphenyl)-9-methyl-3-phenyl-2,3,4,9-tetrahydro$1 H$-carbazole-4-carboxylate (syn-5c):

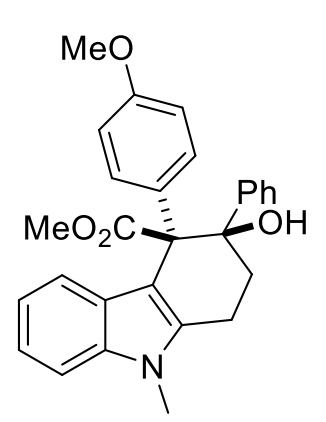

White solid, $85 \%$ yield, ${ }^{1} \mathrm{H}$ NMR (400 MHz, Chloroform- $d$ ) $\delta 7.33$ $(\mathrm{d}, J=8.2 \mathrm{~Hz}, 1 \mathrm{H}), 7.19-7.11(\mathrm{~m}, 4 \mathrm{H}), 7.10-7.00(\mathrm{~m}, 3 \mathrm{H}), 6.92$ $(\mathrm{t}, J=7.5 \mathrm{~Hz}, 1 \mathrm{H}), 6.51-6.41(\mathrm{~m}, 4 \mathrm{H}), 5.89(\mathrm{~s}, 1 \mathrm{H}), 3.76(\mathrm{~s}, 3 \mathrm{H})$, $3.74(\mathrm{~s}, 3 \mathrm{H}), 3.67(\mathrm{~s}, 3 \mathrm{H}), 3.29-3.19(\mathrm{~m}, 1 \mathrm{H}), 2.96-2.84(\mathrm{~m}$, $1 \mathrm{H}), 2.41-2.26(\mathrm{~m}, 2 \mathrm{H}) .{ }^{13} \mathrm{C} \mathrm{NMR}\left(100 \mathrm{MHz}, \mathrm{CDCl}_{3}\right) \delta 176.7$, $158.2,144.6,137.6,137.6,132.4,130.2,127.2,127.2,127.0$, $126.4,120.9,119.7,119.2,111.3,108.8,108.8,77.9,61.3,55.0,52.4,31.8,29.4,20.0$. HRMS(ESI) Calcd. for $\mathrm{C}_{28} \mathrm{H}_{27} \mathrm{NO}_{4}(\mathrm{M}+\mathrm{Na})^{+} 464.1833$, found: 464.1838 .

Methyl 4-(4-fluorophenyl)-3-hydroxy-9-methyl-3-phenyl-2,3,4,9-tetrahydro-1Hcarbazole-4-carboxylate (syn-5d):

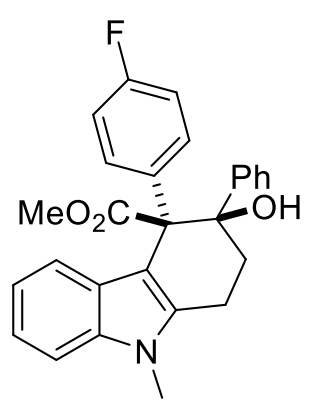

White solid, 57\% yield, ${ }^{1} \mathrm{H}$ NMR (400 MHz, Chloroform- $d$ ) $\delta 7.35$ (d, $J=8.2 \mathrm{~Hz}, 1 \mathrm{H}), 7.20-7.15(\mathrm{~m}, 2 \mathrm{H}), 7.15-7.10(\mathrm{~m}, 2 \mathrm{H}), 7.08$ - $6.99(\mathrm{~m}, 3 \mathrm{H}), 6.96-6.90(\mathrm{~m}, 1 \mathrm{H}), 6.64-6.51(\mathrm{~m}, 4 \mathrm{H}), 5.73(\mathrm{~s}$, $1 \mathrm{H}), 3.77(\mathrm{~s}, 3 \mathrm{H}), 3.73(\mathrm{~s}, 3 \mathrm{H}), 3.28-3.19(\mathrm{~m}, 1 \mathrm{H}), 2.97-2.88(\mathrm{~m}$, $1 \mathrm{H}), 2.49-2.39(\mathrm{~m}, 1 \mathrm{H}), 2.36-2.28(\mathrm{~m}, 1 \mathrm{H}) .{ }^{13} \mathrm{C} \mathrm{NMR}(100 \mathrm{MHz}$, $\left.\mathrm{CDCl}_{3}\right) \delta 176.3,162.8,160.4,144.2,137.7,133.7,132.9,132.8$, 
127.3, 127.1, 126.9, 126.2, 121.1, 119.9, 119.3, 112.9, 112.7, 108.9, 108.5, 77.8, 61.3, 52.4, 32.2, 29.4, 20.0. ${ }^{19} \mathrm{~F}$ NMR (376 MHz, $\left.\mathrm{CDCl}_{3}\right) \delta$-116.38. HRMS(ESI) Calcd.for $\mathrm{C}_{27} \mathrm{H}_{24} \mathrm{FNO}_{3}(\mathrm{M}+\mathrm{Na})^{+}$452.1643, found: 452.1638 .

\section{Methyl 3-hydroxy-9-methyl-3,4-diphenyl-2,3,4,9-tetrahydro-1H-carbazole-4-} carboxylate (syn-5e):

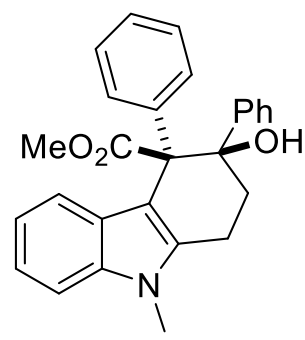

White solid, 91\% yield, ${ }^{1} \mathrm{H}$ NMR (400 MHz, Chloroform- $d$ ) $\delta 7.34$ $(\mathrm{d}, J=8.2 \mathrm{~Hz}, 1 \mathrm{H}), 7.20-6.99(\mathrm{~m}, 8 \mathrm{H}), 6.97-6.84(\mathrm{~m}, 3 \mathrm{H}), 6.62$ (d, $J=7.4 \mathrm{~Hz}, 2 \mathrm{H}), 5.92(\mathrm{~s}, 1 \mathrm{H}), 3.77$ (s, 3H), 3.73 (s, 3H), $3.30-$ $3.18(\mathrm{~m}, 1 \mathrm{H}), 2.99-2.87(\mathrm{~m}, 1 \mathrm{H}), 2.53-2.39(\mathrm{~m}, 1 \mathrm{H}), 2.39-2.30$ $(\mathrm{m}, 1 \mathrm{H}) .{ }^{13} \mathrm{C} \mathrm{NMR}\left(100 \mathrm{MHz}, \mathrm{CDCl}_{3}\right) \delta 176.7,144.4,137.9,137.8$, 137.6, 131.2, 127.1, 127.0, 126.9, 126.6, 126.4, 126.0, 120.9, 120.2, 119.2, 108.8, 108.5, 100.0, 77.9, 61.9, 52.4, 32.3, 29.4, 20.1. HRMS(ESI) Calcd. for $\mathrm{C}_{27} \mathrm{H}_{25} \mathrm{NNaO}_{3}$ $(\mathrm{M}+\mathrm{Na})^{+} 434.1721$, found: 434.1732 .

Methyl 3-hydroxy-9-methyl-4-(naphthalen-1-yl)-3-phenyl-2,3,4,9-tetrahydro-1Hcarbazole-4-carboxylate (syn-5f):

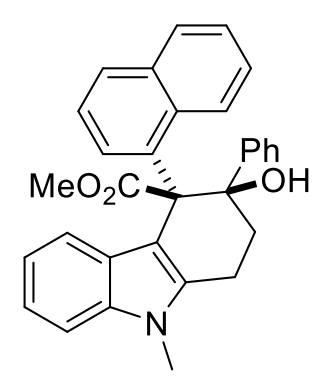

White solid, 82\% yield, ${ }^{1} \mathrm{H}$ NMR (400 MHz, Chloroform- $d$ ) $\delta 7.66$ $(\mathrm{d}, J=8.0 \mathrm{~Hz}, 1 \mathrm{H}), 7.43(\mathrm{~d}, J=8.1 \mathrm{~Hz}, 1 \mathrm{H}), 7.39-7.26(\mathrm{~m}, 4 \mathrm{H})$, $7.22-6.94(\mathrm{~m}, 8 \mathrm{H}), 6.88(\mathrm{t}, J=7.5 \mathrm{~Hz}, 1 \mathrm{H}), 6.62(\mathrm{~d}, J=8.5 \mathrm{~Hz}$, $1 \mathrm{H}), 6.05(\mathrm{~s}, 1 \mathrm{H}), 3.80(\mathrm{~s}, 3 \mathrm{H}), 3.75(\mathrm{~s}, 3 \mathrm{H}), 3.36-3.22(\mathrm{~m}, 1 \mathrm{H})$, $3.02-2.89(\mathrm{~m}, 1 \mathrm{H}), 2.52-2.28(\mathrm{~m}, 2 \mathrm{H}) .{ }^{13} \mathrm{C} \mathrm{NMR}(100 \mathrm{MHz}$, $\left.\mathrm{CDCl}_{3}\right) \delta 176.7,144.5,137.9,137.7,135.9,132.1,131.9,130.6,129.4,128.4,127.2$, 127.1, 127.0, 126.4, 125.8, 125.2, 124.8, 120.9, 119.9, 119.3, 108.8, 108.4, 78.0, 62.1, 52.4, 32.1, 29.5, 20.1, 0.0. HRMS(ESI) Calcd. for $\mathrm{C}_{31} \mathrm{H}_{27} \mathrm{NO}_{3} \mathrm{Na}(\mathrm{M}+\mathrm{Na})^{+}$484.1893, found: 484.1889 .

Methyl 4-(3-chlorophenyl)-3-hydroxy-9-methyl-3-phenyl-2,3,4,9-tetrahydro-1Hcarbazole-4-carboxylate (syn-5g): 


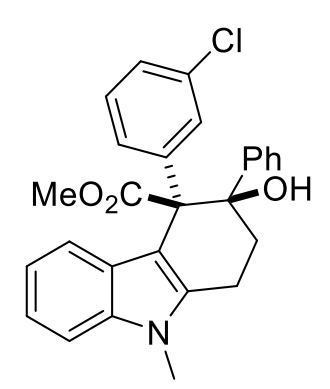

White solid, $53 \%$ yield, ${ }^{1} \mathrm{H}$ NMR (400 MHz, Chloroform- $d$ ) $\delta 7.34$ (d, $J=8.2 \mathrm{~Hz}, 1 \mathrm{H}), 7.20-7.12(\mathrm{~m}, 4 \mathrm{H}), 7.11-6.98(\mathrm{~m}, 4 \mathrm{H}), 6.94$ (t, $J=7.2 \mathrm{~Hz}, 1 \mathrm{H}), 6.83(\mathrm{t}, J=8.1 \mathrm{~Hz}, 1 \mathrm{H}), 6.71-6.38(\mathrm{~m}, 2 \mathrm{H})$, $5.75(\mathrm{~s}, 1 \mathrm{H}), 3.76(\mathrm{~s}, 3 \mathrm{H}), 3.73(\mathrm{~s}, 3 \mathrm{H}), 3.27-3.19(\mathrm{~m}, 1 \mathrm{H}), 2.97-$ $2.87(\mathrm{~m}, 1 \mathrm{H}), 2.58-2.38(\mathrm{~m}, 1 \mathrm{H}), 2.35-2.27(\mathrm{~m}, 1 \mathrm{H}) .{ }^{13} \mathrm{C} \mathrm{NMR}$ $\left(100 \mathrm{MHz}, \mathrm{CDCl}_{3}\right) \delta 175.9,143.9,140.2,137.8,137.7,132.0,131.5,129.2,127.3$, 127.2, 127.2, 126.9, 126.8, 126.1, 121.1, 119.7, 119.4, 108.9, 107.9, 77.9, 61.8, 52.5, 32.1, 29.5, 20.0. HRMS(ESI) Calcd. for $\mathrm{C}_{27} \mathrm{H}_{24} \mathrm{ClNO}_{3} \mathrm{Na}(\mathrm{M}+\mathrm{Na})^{+} 468.1359$, found: 468.1342 .

Methyl 4-(3,4-dichlorophenyl)-3-hydroxy-9-methyl-3-phenyl-2,3,4,9-tetrahydro$1 H$-carbazole-4-carboxylate (syn-5h):

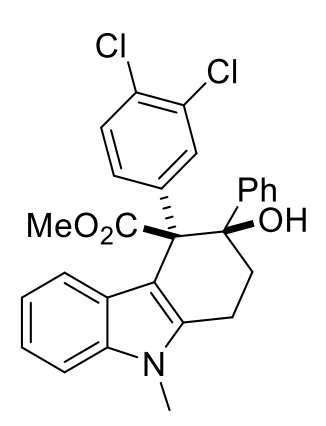

White solid, 60\% yield, ${ }^{1} \mathrm{H}$ NMR (400 MHz, Chloroform- $d$ ) $\delta 7.36$ $(\mathrm{d}, J=8.2 \mathrm{~Hz}, 1 \mathrm{H}), 7.22-7.13(\mathrm{~m}, 4 \mathrm{H}), 7.11-7.02(\mathrm{~m}, 2 \mathrm{H}), 7.00$ $-6.92(\mathrm{~m}, 3 \mathrm{H}), 6.62(\mathrm{~s}, 1 \mathrm{H}), 6.46(\mathrm{~d}, J=7.6 \mathrm{~Hz}, 1 \mathrm{H}), 5.60(\mathrm{~s}, 1 \mathrm{H})$, $3.78(\mathrm{~s}, 3 \mathrm{H}), 3.74(\mathrm{~s}, 3 \mathrm{H}), 3.28-3.19(\mathrm{~m}, 1 \mathrm{H}), 2.97-2.88(\mathrm{~m}, 1 \mathrm{H})$, $2.53-2.40(\mathrm{~m}, 1 \mathrm{H}), 2.33-2.24(\mathrm{~m}, 1 \mathrm{H}) .{ }^{13} \mathrm{C}$ NMR (100 MHz, $\left.\mathrm{CDCl}_{3}\right) \delta 175.4,143.6,138.5,137.7,137.7,133.4,130.8,130.4$, 130.1, 127.8, 127.5, 127.4, 126.8, 125.9, 121.2, 119.6, 119.5, 109.0, 107.6, 77.8, 61.5, 52.6, 32.1, 29.5, 19.9. HRMS(ESI) Calcd. for $\mathrm{C}_{27} \mathrm{H}_{23} \mathrm{Cl}_{2} \mathrm{NO}_{3}(\mathrm{M}+\mathrm{Na})^{+}$502.0972, found: 502.0953 .

Methyl 3-hydroxy-4-(3-methoxyphenyl)-9-methyl-3-phenyl-2,3,4,9-tetrahydro$1 H$-carbazole-4-carboxylate (syn-5i):

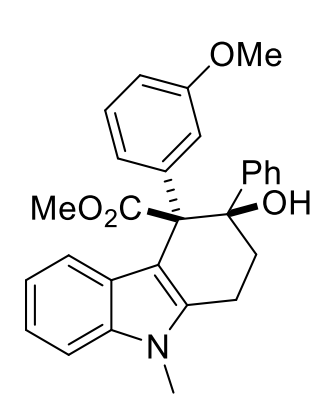

White solid, 51\% yield, ${ }^{1} \mathrm{H}$ NMR (400 MHz, Chloroform- $d$ ) $\delta 7.33$ $(\mathrm{d}, J=8.2 \mathrm{~Hz}, 1 \mathrm{H}), 7.14(\mathrm{q}, J=7.0 \mathrm{~Hz}, 4 \mathrm{H}), 7.10-7.01(\mathrm{~m}, 3 \mathrm{H})$, $6.92(\mathrm{t}, J=7.5 \mathrm{~Hz}, 1 \mathrm{H}), 6.84(\mathrm{t}, J=8.0 \mathrm{~Hz}, 1 \mathrm{H}), 6.65-6.59(\mathrm{~m}$, $1 \mathrm{H}), 6.25(\mathrm{~d}, J=7.6 \mathrm{~Hz}, 1 \mathrm{H}), 6.16(\mathrm{~s}, 1 \mathrm{H}), 5.86$ (s, 1H), 3.77 (s, $3 \mathrm{H}), 3.74(\mathrm{~s}, 3 \mathrm{H}), 3.43(\mathrm{~s}, 3 \mathrm{H}), 3.28-3.19(\mathrm{~m}, 1 \mathrm{H}), 2.96-2.89(\mathrm{~m}$, $1 \mathrm{H}), 2.45-2.35(\mathrm{~m}, 2 \mathrm{H}) .{ }^{13} \mathrm{C} \mathrm{NMR}\left(100 \mathrm{MHz}, \mathrm{CDCl}_{3}\right) \delta 176.4$, 
157.4, 144.5, 139.6, 137.6, 137.6, 127.1, 127.1, 126.9, 126.8, 126.4, 123.7, 120.9, 120.0, $119.2,117.4,112.4,108.8,108.5,77.9,61.9,54.8,52.3,32.1,29.4,20.0,0.0$. HRMS(ESI) Calcd. for $\mathrm{C}_{28} \mathrm{H}_{27} \mathrm{NO}_{4} \mathrm{Na}(\mathrm{M}+\mathrm{Na})^{+} 464.1837$, found: 464.1838 .

Methyl 3-(4-chlorophenyl)-3-hydroxy-9-methyl-4-phenyl-2,3,4,9-tetrahydro-1Hcarbazole-4-carboxylate (syn-5j):

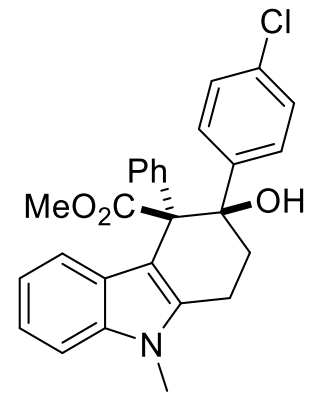

White solid, 84\% yield, ${ }^{1} \mathrm{H}$ NMR (400 MHz, Chloroform- $d$ ) $\delta$ $7.34(\mathrm{~d}, J=8.2 \mathrm{~Hz}, 1 \mathrm{H}), 7.19-7.15(\mathrm{~m}, 1 \mathrm{H}), 7.09-7.02(\mathrm{~m}$, 4H), $7.02-6.86(\mathrm{~m}, 5 \mathrm{H}), 6.70(\mathrm{~d}, J=7.5 \mathrm{~Hz}, 2 \mathrm{H}), 5.84(\mathrm{~s}, 1 \mathrm{H})$, $3.76(\mathrm{~s}, 3 \mathrm{H}), 3.70(\mathrm{~s}, 3 \mathrm{H}), 3.25-3.16(\mathrm{~m}, 1 \mathrm{H}), 2.94-2.84(\mathrm{~m}$, $1 \mathrm{H}), 2.63-2.39(\mathrm{~m}, 1 \mathrm{H}), 2.33-2.25(\mathrm{~m}, 1 \mathrm{H}) .{ }^{13} \mathrm{C}$ NMR $(100$ $\left.\mathrm{MHz}, \mathrm{CDCl}_{3}\right) \delta 176.6,142.8,137.7,137.7,137.5,132.7,131.0,128.7,128.5,127.7$, 127.1, 126.8, 126.3, 126.3, 121.1, 120.6, 119.2, 108.8, 108.3, 77.8, 61.7, 52.4, 32.8, 29.4, 20.2, 0.0. HRMS(ESI) Calcd. for $\mathrm{C}_{27} \mathrm{H}_{24} \mathrm{ClNO}_{3} \mathrm{Na}(\mathrm{M}+\mathrm{Na})^{+} 468.1360$, found: 468.1342 .

Methyl 3-hydroxy-3-(4-methoxyphenyl)-9-methyl-4-phenyl-2,3,4,9-tetrahydro$1 H$-carbazole-4-carboxylate (syn-5k):

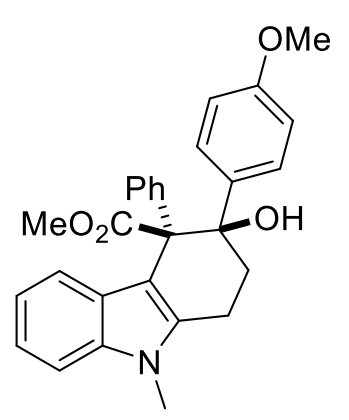

White solid, 66\% yield, ${ }^{1} \mathrm{H}$ NMR (400 MHz, Chloroform- $d$ ) $\delta 7.34$ $(\mathrm{d}, J=8.2 \mathrm{~Hz}, 1 \mathrm{H}), 7.18-7.13(\mathrm{~m}, 1 \mathrm{H}), 7.08-7.02(\mathrm{~m}, 2 \mathrm{H}), 6.98$ - $6.87(\mathrm{~m}, 5 \mathrm{H}), 6.63(\mathrm{t}, J=8.5 \mathrm{~Hz}, 4 \mathrm{H}), 5.84(\mathrm{~s}, 1 \mathrm{H}), 3.76(\mathrm{~s}, 3 \mathrm{H})$, $3.75(\mathrm{~s}, 3 \mathrm{H}), 3.73(\mathrm{~s}, 3 \mathrm{H}), 3.27-3.18(\mathrm{~m}, 1 \mathrm{H}), 2.95-2.86(\mathrm{~m}, 1 \mathrm{H})$, $2.46-2.25(\mathrm{~m}, 2 \mathrm{H}) .{ }^{13} \mathrm{C} \mathrm{NMR}\left(100 \mathrm{MHz}, \mathrm{CDCl}_{3}\right) \delta 176.7,158.4$, $138.1,137.8,137.7,136.7,131.2,130.3,128.2,126.6,126.4,126.0$, 120.9, 120.1, 119.2, 112.4, 108.8, 108.6, 77.8, 62.0, 55.2, 52.3, 32.4, 29.4, 20.1. HRMS(ESI) Calcd. for $\mathrm{C}_{28} \mathrm{H}_{27} \mathrm{NO}_{4} \mathrm{Na}(\mathrm{M}+\mathrm{Na})^{+} 464.1816$, found: 464.1838 . 
Methyl 3-hydroxy-9-methyl-4-phenyl-3-(4-(trifluoromethyl)phenyl)-2,3,4,9tetrahydro-1H-carbazole-4-carboxylate (syn-5I):

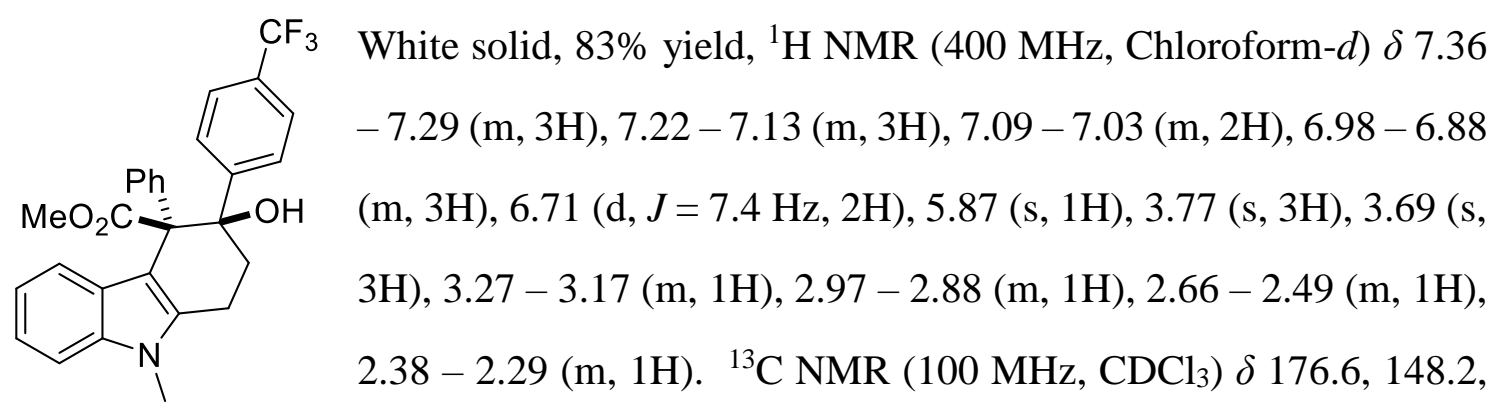
137.8, 137.6, 137.3, 130.8, 129.0, 128.7, 128.9 (q, $J=97.0 \mathrm{~Hz}) 127.3,126.9,126.3$, 126.3, 125.6, 124.0, 123.9, 123.9, 123.8, 123.9 (q, $J=3.9 \mathrm{~Hz}) 122.9,121.1,120.9$, 119.3, 108.8, 108.2, 78.0, 61.6, 52.4, 32.9, 29.7, 29.5, 20.2, 0.0. ${ }^{19} \mathrm{~F}$ NMR (376 MHz, $\left.\mathrm{CDCl}_{3}\right) \delta$-62.47. HRMS(ESI) Calcd. for $\mathrm{C}_{28} \mathrm{H}_{24} \mathrm{FNO}_{3} \mathrm{Na}(\mathrm{M}+\mathrm{Na})^{+}$502.1616, found: 502.1606.

Benzyl 3-hydroxy-9-methyl-3,4-diphenyl-2,3,4,9-tetrahydro-1H-carbazole-4carboxylate (syn-5m):

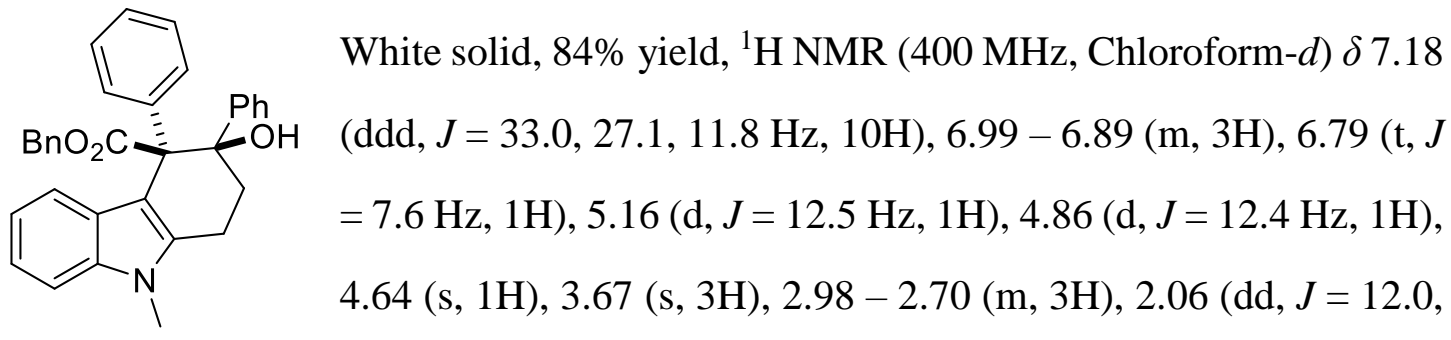

$5.1 \mathrm{~Hz}, 1 \mathrm{H}), 0.80$ (s, 3H). ${ }^{13} \mathrm{C}$ NMR (100 MHz, $\left.\mathrm{CDCl}_{3}\right) \delta 176.3,137.8,137.2,137.1$, 135.2, 129.9, 128.1, 127.9, 126.9, 126.8, 126.7, 123.5, 120.7, 118.6, 108.4, 108.2, 77.4, 77.3, 77.1, 76.7, 74.6, 66.8, 60.5, 35.2, 29.3, 23.2, 21.2. HRMS(ESI) Calcd. for $\mathrm{C}_{28} \mathrm{H}_{27} \mathrm{NO}_{3} \mathrm{Na}(\mathrm{M}+\mathrm{Na})^{+} 448.1898$, found: 448.1889.

Benzyl 3-hydroxy-9-methyl-3-phenyl-4-(p-tolyl)-2,3,4,9-tetrahydro-1H-carbazole -4-carboxylate (syn-5n): 


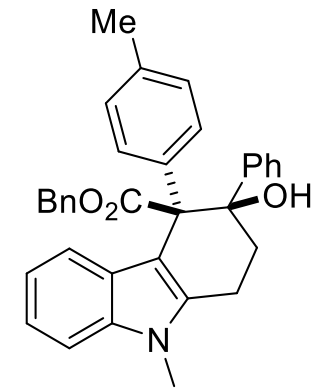

White solid, $88 \%$ yield, ${ }^{1} \mathrm{H}$ NMR (400 MHz, Chloroform- $d$ ) $\delta 7.28$ - $7.21(\mathrm{~m}, 2 \mathrm{H}), 7.21-7.08(\mathrm{~m}, 4 \mathrm{H}), 7.03-6.91(\mathrm{~m}, 7 \mathrm{H}), 6.80$ (t, $J=7.7 \mathrm{~Hz}, 1 \mathrm{H}), 5.13(\mathrm{~d}, J=12.6 \mathrm{~Hz}, 1 \mathrm{H}), 4.87(\mathrm{~d}, J=12.6 \mathrm{~Hz}$, $1 \mathrm{H}), 4.64(\mathrm{~s}, 1 \mathrm{H}), 3.65(\mathrm{~s}, 3 \mathrm{H}), 2.95-2.67(\mathrm{~m}, 3 \mathrm{H}), 2.32(\mathrm{~s}, 3 \mathrm{H})$, $2.09-2.01(\mathrm{~m}, 1 \mathrm{H}), 0.79$ (s, 3H). ${ }^{13} \mathrm{C} \mathrm{NMR}\left(100 \mathrm{MHz}, \mathrm{CDCl}_{3}\right) \delta$ 176.5, 137.7, 137.1, 136.4, 135.3, 134.0, 129.7, 128.1, 128.1, 127.9, 127.6, 126.8, 123.6, 120.7, 118.6, 108.4, 108.3, 77.4, 77.1, 76.8, 74.6, 66.8, 60.1, 35.1, 29.3, 23.2, 21.2, 21.1. HRMS(ESI) Calcd. for $\mathrm{C}_{29} \mathrm{H}_{29} \mathrm{NO}_{3} \mathrm{Na}(\mathrm{M}+\mathrm{Na})^{+} 462.1721$, found: 462.1736 .

Benzyl 4-(4-fluorophenyl)-3-hydroxy-9-methyl-3-phenyl-2,3,4,9-tetrahydro-1Hcarbazole-4-carboxylate (syn-5o):

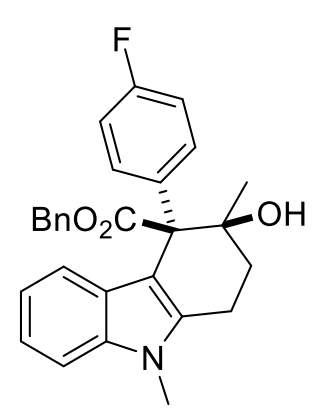

White solid, 91\% yield, ${ }^{1} \mathrm{H}$ NMR (400 MHz, Chloroform- $d$ ) $\delta 7.22$ $-7.08(\mathrm{~m}, 4 \mathrm{H}), 7.05$ (t, $J=7.7 \mathrm{~Hz}, 1 \mathrm{H}), 6.97$ (t, $J=6.9 \mathrm{~Hz}, 2 \mathrm{H})$, $6.90(\mathrm{~d}, J=7.4 \mathrm{~Hz}, 2 \mathrm{H}), 6.84(\mathrm{~d}, J=8.2 \mathrm{~Hz}, 1 \mathrm{H}), 6.82-6.69(\mathrm{~m}$, $3 \mathrm{H}), 5.09(\mathrm{~d}, J=12.3 \mathrm{~Hz}, 1 \mathrm{H}), 4.75(\mathrm{~d}, J=12.4 \mathrm{~Hz}, 1 \mathrm{H}), 4.48(\mathrm{~s}$, $1 \mathrm{H}), 3.59$ (s, 3H), $2.91-2.74(\mathrm{~m}, 2 \mathrm{H}), 2.68(\mathrm{dd}, J=15.3,8.6 \mathrm{~Hz}$, 1H), $2.02-1.93(\mathrm{~m}, 1 \mathrm{H}), 0.70(\mathrm{~s}, 3 \mathrm{H}) .{ }^{13} \mathrm{C}$ NMR $(100 \mathrm{MHz}$, $\left.\mathrm{CDCl}_{3}\right) \delta 175.0,162.0,159.6,136.7,136.2,134.0,131.9,131.9,130.4,130.3,127.2$, 127.2, 127.0, 125.4, 122.2, 119.8, 117.7, 112.8, 112.6, 107.4, 106.9, 76.3, 76.0, 75.7, 73.5, 65.9, 58.8, 34.0, 28.2, 22.1, 20.1. ${ }^{19} \mathrm{~F}$ NMR $\left(376 \mathrm{MHz}, \mathrm{CDCl}_{3}\right) \delta-116.38$. HRMS(ESI) Calcd. for $\mathrm{C}_{33} \mathrm{H}_{28} \mathrm{NO}_{3} \mathrm{NaF}(\mathrm{M}+\mathrm{Na})^{+} 466.1485$, found: 466.1502 .

\section{Ethyl 3-hydroxy-9-methyl-3,4-diphenyl-2,3,4,9-tetrahydro-1H-carbazole-4} carboxylate (syn-5p): 


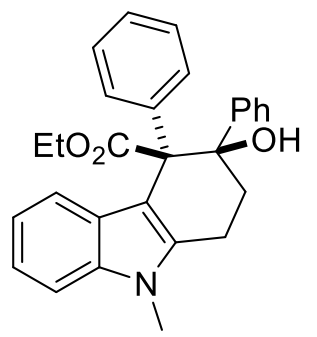

White solid, $61 \%$ yield, ${ }^{1} \mathrm{H}$ NMR ( $400 \mathrm{MHz}$, Chloroform- $d$ ) $\delta 7.34$ $(\mathrm{d}, J=8.2 \mathrm{~Hz}, 1 \mathrm{H}), 7.19-7.01(\mathrm{~m}, 8 \mathrm{H}), 6.90(\mathrm{t}, J=7.6 \mathrm{~Hz}, 3 \mathrm{H})$, $6.64(\mathrm{~d}, J=7.6 \mathrm{~Hz}, 2 \mathrm{H}), 6.01(\mathrm{~s}, 1 \mathrm{H}), 4.36-4.26(\mathrm{~m}, 1 \mathrm{H}), 4.18-$ $4.09(\mathrm{~m}, 1 \mathrm{H}), 3.78(\mathrm{~s}, 3 \mathrm{H}), 3.29-3.19(\mathrm{~m}, 1 \mathrm{H}), 2.98-2.88(\mathrm{~m}$, $1 \mathrm{H}), 2.56-2.43(\mathrm{~m}, 1 \mathrm{H}), 2.38-2.29(\mathrm{~m}, 1 \mathrm{H}), 1.16(\mathrm{t}, J=7.1 \mathrm{~Hz}$,

$3 \mathrm{H}) .{ }^{13} \mathrm{C}$ NMR (100 MHz, Chloroform- $d$ ) $\delta$ 176.2, 137.9, 137.8, 137.7, 131.2, 127.1, 127.0, 126.8, 126.5, 126.4, 125.9, 120.9, 118.9, 108.7, 77.9, 61.8, 32.5, 29.4, 20.2, 13.9, 8.6. HRMS(ESI) Calcd. for $\mathrm{C}_{28} \mathrm{H}_{27} \mathrm{NO}_{3}(\mathrm{M}+\mathrm{Na})^{+} 448.1898$, found: 448.1889 .

\section{Methyl 4-(3,4-dimethoxyphenyl)-3-hydroxy-9-methyl-3-phenyl-2,3,4,9-tetrahydr} -o-1H-carbazole-4-carboxylate (syn-5q):

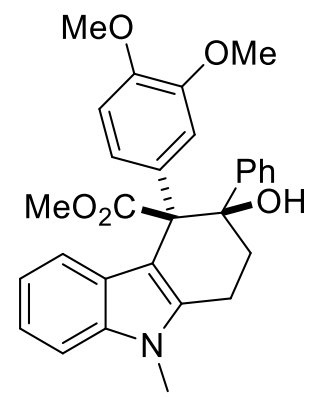

White solid, 83\% yield, ${ }^{1} \mathrm{H}$ NMR (400 MHz, Chloroform- $d$ ) $\delta 7.34$ $(\mathrm{d}, J=8.2 \mathrm{~Hz}, 1 \mathrm{H}), 7.21-7.11(\mathrm{~m}, 8 \mathrm{H}), 7.10-7.07(\mathrm{~m}, 1 \mathrm{H}), 7.03$ $(\mathrm{d}, J=8.0 \mathrm{~Hz}, 1 \mathrm{H}), 6.97-6.88(\mathrm{~m}, 2 \mathrm{H}), 6.46(\mathrm{~d}, J=8.5 \mathrm{~Hz}, 1 \mathrm{H})$, $6.25(\mathrm{~d}, J=7.8 \mathrm{~Hz}, 1 \mathrm{H}), 6.09-5.91(\mathrm{~m}, 2 \mathrm{H}), 5.72(\mathrm{~s}, 1 \mathrm{H}), 3.77(\mathrm{~s}$, 3H), 3.76 (s, 3H), 3.74 (s, 3H), 3.29 (s, 3H), $3.27-3.19$ (m, 1H), $2.97-2.85(\mathrm{~m}, 1 \mathrm{H}), 2.46-2.38(\mathrm{~m}, 1 \mathrm{H}), 2.37-2.18(\mathrm{~m}, 1 \mathrm{H}) .{ }^{13} \mathrm{C} \mathrm{NMR}(100 \mathrm{MHz}$, $\left.\mathrm{CDCl}_{3}\right) \delta 176.4,147.7,145.9,144.7,137.6,137.4,130.6,127.3,127.3,127.0,126.5$, $123.2,120.9,119.6,119.3,115.7,108.8,108.8,108.7,77.9,61.5,55.5,55.1,52.4,31.8$, 29.4, 19.8, 0.0. HRMS(ESI) Calcd. for $\mathrm{C}_{29} \mathrm{H}_{29} \mathrm{NO}_{5} \mathrm{Na}(\mathrm{M}+\mathrm{Na})^{+}$494.1958, found: 494.1943.

Methyl 9-benzyl-3-hydroxy-3,4-diphenyl-2,3,4,9-tetrahydro-1H-carbazole-4carboxylate (syn-5r):

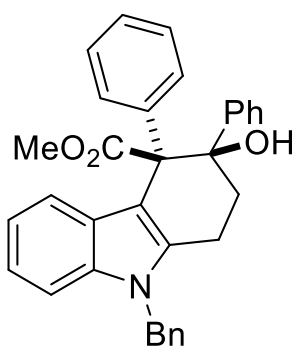

White solid, 60\% yield, ${ }^{1} \mathrm{H}$ NMR (400 MHz, Chloroform- $d$ ) $\delta$ $7.39-7.26(\mathrm{~m}, 4 \mathrm{H}), 7.19-6.97(\mathrm{~m}, 10 \mathrm{H}), 6.93(\mathrm{t}, J=7.4 \mathrm{~Hz}$, $3 \mathrm{H}), 6.66(\mathrm{~d}, J=7.2 \mathrm{~Hz}, 2 \mathrm{H}), 5.89(\mathrm{~s}, 1 \mathrm{H}), 5.45(\mathrm{~d}, J=16.9 \mathrm{~Hz}$, 1H), $5.35(\mathrm{~d}, J=16.8 \mathrm{~Hz}, 1 \mathrm{H}), 3.74(\mathrm{~s}, 3 \mathrm{H}), 3.24-3.11(\mathrm{~m}, 1 \mathrm{H})$, $2.90-2.76(\mathrm{~m}, 1 \mathrm{H}), 2.62-2.36(\mathrm{~m}, 1 \mathrm{H}), 2.36-2.24(\mathrm{~m}, 1 \mathrm{H}) .{ }^{13} \mathrm{C}$ 
NMR (100 MHz, $\left.\mathrm{CDCl}_{3}\right) \delta 176.7,144.2,137.8,137.7,137.5,131.1,128.9,127.5,127.1$, 126.97, 126.9, 126.6, 126.6, 126.2, 126.1, 121.3, 120.5, 119.5, 109.4, 109.3, 77.9, 61.8, 52.4, 46.5, 32.4, 20.2, 0.0. HRMS(ESI) Calcd. for $\mathrm{C}_{33} \mathrm{H}_{29} \mathrm{NO}_{3} \mathrm{Na}(\mathrm{M}+\mathrm{Na})^{+}$510.2066, found: 510.2045 .

Methyl 3-hydroxy-3,9-dimethyl-4-phenyl-2,3,4,9-tetrahydro-1H-carbazole-4carboxylate (syn-5s):

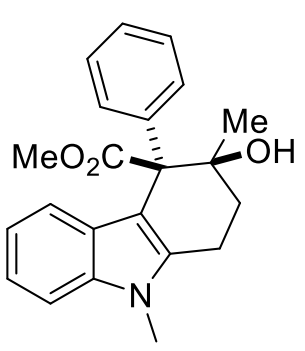

White solid, $80 \%$ yield, ${ }^{1} \mathrm{H}$ NMR (400 MHz, Chloroform- $d$ ) $\delta 7.32$ $-7.22(\mathrm{~m}, 5 \mathrm{H}), 7.20-7.10(\mathrm{~m}, 3 \mathrm{H}), 7.00(\mathrm{~d}, J=8.0 \mathrm{~Hz}, 1 \mathrm{H}), 6.90$ (t, $J=7.5 \mathrm{~Hz}, 1 \mathrm{H}), 4.68(\mathrm{~s}, 1 \mathrm{H}), 3.67$ (s, 3H), 3.56 (s, 3H), $2.97-$ $2.90(\mathrm{~m}, 1 \mathrm{H}), 2.89-2.71(\mathrm{~m}, 2 \mathrm{H}), 2.10-2.03(\mathrm{~m}, 1 \mathrm{H}), 0.79(\mathrm{~s}$,

3H). ${ }^{13} \mathrm{C} \mathrm{NMR}\left(100 \mathrm{MHz}, \mathrm{CDCl}_{3}\right) \delta 177.2,137.7,137.5,137.3$, 129.7, 127.0, 126.8, 126.7, 123.1, 120.7, 118.7, 108.5, 108.2, 74.5, 60.3, 52.0, 35.2, 29.3, 23.2, 21.2, 0.0. HRMS(ESI) Calcd. for $\mathrm{C}_{22} \mathrm{H}_{23} \mathrm{NO}_{3}(\mathrm{M}+\mathrm{Na})^{+}$372.1566, found: 372.1576.

Methyl 3-ethyl-3-hydroxy-9-methyl-4-phenyl-2,3,4,9-tetrahydro-1H-carbazole-4carboxylate (syn-5t):<smiles>CCC1(O)CCc2c(c3ccccc3n2C)[C@]1(c1ccccc1)C(C)(O)c1ccccc1</smiles>

White solid, 66\% yield, ${ }^{1} \mathrm{H}$ NMR (400 MHz, Chloroform- $d$ ) $\delta 7.32$ $-7.22(\mathrm{~m}, 5 \mathrm{H}), 7.21-7.07$ (m, 3H), 6.99 (d, J = 8.0 Hz, 1H), 6.89 (t, $J=7.5 \mathrm{~Hz}, 1 \mathrm{H}), 4.61(\mathrm{~s}, 1 \mathrm{H}), 3.67$ (s, 3H), 3.55 (s, 3H), $2.92-$ $2.81(\mathrm{~m}, 1 \mathrm{H}), 2.79-2.69(\mathrm{~m}, 1 \mathrm{H}), 2.68-2.57(\mathrm{~m}, 1 \mathrm{H}), 2.33-2.22$ (m, 1H), $1.38-1.28(\mathrm{~m}, 2 \mathrm{H}), 0.83(\mathrm{t}, J=7.3 \mathrm{~Hz}, 3 \mathrm{H}) .{ }^{13} \mathrm{C} \mathrm{NMR}$ $\left(100 \mathrm{MHz} \mathrm{CDCl}_{3}\right) \delta 177.3,137.9,137.6,137.4,129.9,127.0,126.9,126.8,123.2$, 120.6, 118.6, 108.4, 107.9, 76.0, 61.2, 52.0, 29.7, 29.3, 26.5, 21.0, 7.5, -0.0. HRMS(ESI) Calcd. for $\mathrm{C}_{23} \mathrm{H}_{25} \mathrm{NO}_{3}(\mathrm{M}+\mathrm{Na})^{+}$386.1725, found: 386.1732 .

Methyl 3-butyl-3-hydroxy-9-methyl-4-phenyl-2,3,4,9-tetrahydro-1H-carbazole-4carboxylate (syn-5u): 


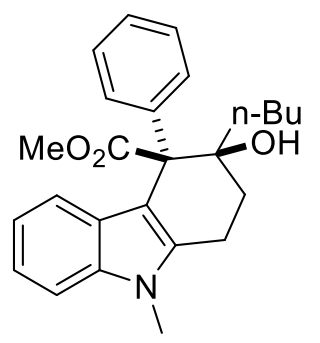

White solid, 65\% yield, ${ }^{1} \mathrm{H}$ NMR (400 MHz, Chloroform- $d$ ) $\delta 7.31$ $-7.22(\mathrm{~m}, 4 \mathrm{H}), 7.21-7.07(\mathrm{~m}, 3 \mathrm{H}), 6.98(\mathrm{~d}, J=8.0 \mathrm{~Hz}, 1 \mathrm{H}), 6.89$ (t, $J=7.4 \mathrm{~Hz}, 1 \mathrm{H}), 4.66(\mathrm{~s}, 1 \mathrm{H}), 3.67(\mathrm{~s}, 3 \mathrm{H}), 3.55$ (s, 3H), $2.92-$ $2.82(\mathrm{~m}, 1 \mathrm{H}), 2.78-2.68(\mathrm{~m}, 1 \mathrm{H}), 2.67-2.57(\mathrm{~m}, 1 \mathrm{H}), 2.30-2.21$ $(\mathrm{m}, 1 \mathrm{H}), 1.43-1.16(\mathrm{~m}, 4 \mathrm{H}), 1.12-1.01(\mathrm{~m}, 2 \mathrm{H}), 0.75(\mathrm{t}, J=7.3$

$\mathrm{Hz}, 3 \mathrm{H}), 0.56(\mathrm{t}, J=11.8 \mathrm{~Hz}, 1 \mathrm{H}) .{ }^{13} \mathrm{C} \mathrm{NMR}\left(100 \mathrm{MHz}, \mathrm{CDCl}_{3}\right) \delta 177.4,137.8,137.6$, $137.3,129.9,126.9,126.8,123.3,120.6,118.6,108.4,108.0,76.0,61.2,52.0,33.7$, 30.5, 29.3, 25.4, 23.3, 21.1, 14.1, -0.0. HRMS(ESI) Calcd. for $\mathrm{C}_{25} \mathrm{H}_{29} \mathrm{NO}_{3} \mathrm{Na}(\mathrm{M}+\mathrm{Na})^{+}$ 414.2050, found: 414.2045 .

\section{(5S,8S,10S,13S,14S)-10,13-dimethyl-17-oxohexadecahydro-1H-cyclopenta[a]}

phenanthren-3-yl-3-hydroxy-3,9-dimethyl-4-phenyl-2,3,4,9-tetrahydro-1Hcarbazole-4-carboxylate (syn-5v):

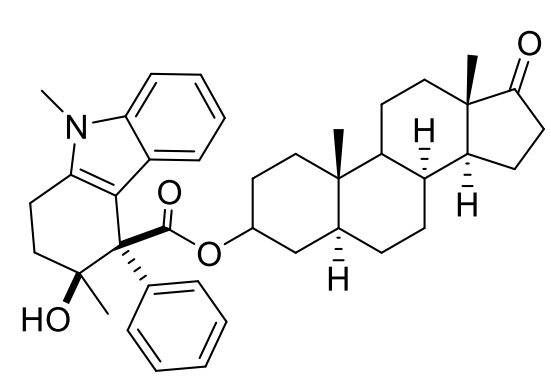

White solid, $83 \%$ yield, ${ }^{1} \mathrm{H}$ NMR (400 MHz, Chloroform- $d$ ) $\delta 7.48-7.26(\mathrm{~m}, 3 \mathrm{H}), 7.23-7.09$ $(\mathrm{m}, 4 \mathrm{H}), 7.05(\mathrm{~d}, J=8.0 \mathrm{~Hz}, 1 \mathrm{H}), 6.88(\mathrm{t}, J=7.8$ $\mathrm{Hz}, 1 \mathrm{H}), 4.63$ (s, 1H), 3.67 (s, 3H), 2.92 (d, $J=10.2$ $\mathrm{Hz}, 1 \mathrm{H}), 2.82(\mathrm{~d}, J=11.0 \mathrm{~Hz}, 2 \mathrm{H}), 2.42(\mathrm{dd}, J=$ 18.3, 8.7 Hz, 1H), $2.26(\mathrm{dd}, J=22.7,13.7 \mathrm{~Hz}, 1 \mathrm{H}), 2.09-2.01(\mathrm{~m}, 2 \mathrm{H}), 1.96-1.84$ $(\mathrm{m}, 2 \mathrm{H}), 1.82-1.60(\mathrm{~m}, 6 \mathrm{H}), 1.53-1.39(\mathrm{~m}, 5 \mathrm{H}), 1.22-1.16(\mathrm{~m}, 3 \mathrm{H}), 1.08(\mathrm{q}, J=$ 11.6, $9.8 \mathrm{~Hz}, 3 \mathrm{H}), 0.96$ (q, $J=11.9,10.3 \mathrm{~Hz}, 3 \mathrm{H}), 0.81$ (s, 3H), 0.75 (s, 3H), 0.68 (s, 3H). ${ }^{13} \mathrm{C}$ NMR (100 MHz, CDCl3) $\delta$ 176.0, 176.0, 138.0, 137.7, 137.2, 137.1, 129.9, $126.8,126.7,126.7,126.6,123.8,123.7,120.6,118.2,118.2,108.4,108.3,108.3,77.4$, $77.1,76.8,74.8,74.8,74.5,60.4,54.3,51.4,51.3,47.8,44.6,36.6,36.5,35.9,35.6$, $35.6,35.2,35.0,34.9,33.3,31.5,30.8,30.7,29.3,28.3,28.1,26.9,23.4,21.8,21.7$, 21.2, 20.4, 20.4, 13.8, 12.2, 12.1. HRMS(ESI) Calcd. for $\mathrm{C}_{40} \mathrm{H}_{49} \mathrm{NO}_{4} \mathrm{Na}(\mathrm{M}+\mathrm{Na})^{+}$ 630.3544, found: 630.3559 . 
(2S,5R)-2-isopropyl-5-methylcyclohexyl-3-hydroxy-3,9-dimethyl-4-phenyl-2,3,4,9 -tetrahydro-1H-carbazole-4-carboxylate (syn-5w):

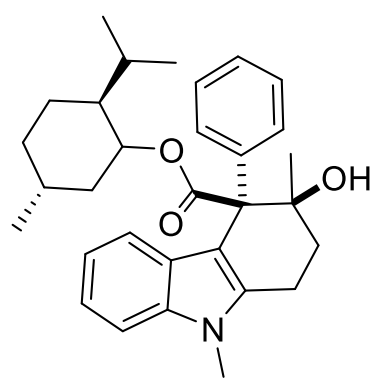

White solid, 77\% yield, ${ }^{1} \mathrm{H}$ NMR (400 MHz, Chloroform- $d$ ) $\delta$ $7.32-7.25(\mathrm{~m}, 4 \mathrm{H}), 7.22(\mathrm{~d}, J=8.4 \mathrm{~Hz}, 2 \mathrm{H}), 7.09(\mathrm{~d}, J=7.5 \mathrm{~Hz}$, $1 \mathrm{H}), 6.91(\mathrm{~d}, J=8.1 \mathrm{~Hz}, 1 \mathrm{H}), 6.85(\mathrm{t}, J=7.6 \mathrm{~Hz}, 1 \mathrm{H}), 5.09(\mathrm{~s}$, $1 \mathrm{H}), 4.63(\mathrm{td}, J=10.8,9.6,5.2 \mathrm{~Hz}, 1 \mathrm{H}), 3.66(\mathrm{~s}, 3 \mathrm{H}), 2.98-2.77$ (m, 2H), $2.72-2.62(\mathrm{~m}, 1 \mathrm{H}), 2.08(\mathrm{dd}, J=12.9,5.8 \mathrm{~Hz}, 1 \mathrm{H})$, $1.98(\mathrm{~d}, J=12.7 \mathrm{~Hz}, 1 \mathrm{H}), 1.56(\mathrm{~s}, 3 \mathrm{H}), 1.31(\mathrm{dd}, J=30.0,11.0 \mathrm{~Hz}, 4 \mathrm{H}), 0.88(\mathrm{~d}, J=$ $6.6 \mathrm{~Hz}, 3 \mathrm{H}), 0.83(\mathrm{~s}, 3 \mathrm{H}), 0.73(\mathrm{dt}, J=35.3,13.9 \mathrm{~Hz}, 4 \mathrm{H}), 0.28(\mathrm{~s}, 3 \mathrm{H}), 0.02(\mathrm{~s}, 2 \mathrm{H})$. ${ }^{13} \mathrm{C}$ NMR (100 MHz, CDCl3) $\delta$ 176.5, 137.8, 137.1, 136.5, 129.9, 127.2, 127.2, 126.9, 126.8, 123.6, 120.6, 118.4, 109.1, 109.1, 108.1, 77.4, 77.0, 76.7, 74.6, 74.4, 60.2, 46.6, 39.8, 35.4, 34.0, 31.4, 29.2 24.8, 22.8, 22.6, 22.0, 21.2, 20.1, 15.4. HRMS(ESI) Calcd. for $\mathrm{C}_{31} \mathrm{H}_{39} \mathrm{NO}_{3} \mathrm{Na}(\mathrm{M}+\mathrm{Na})+496.2788$, found: 496.2828.

(3aR,5R,6R,6aR)-5-((S)-2,2-dimethyl-1,3-dioxolan-4-yl)-2,2-dimethyltetrahydrofuro[2,3-d][1,3]dioxol-6-yl-3-hydroxy-3,9-dimethyl-4-phenyl-2,3,4,9-tetrahydro$1 H$-carbazole-4-carboxylate (syn-5x):
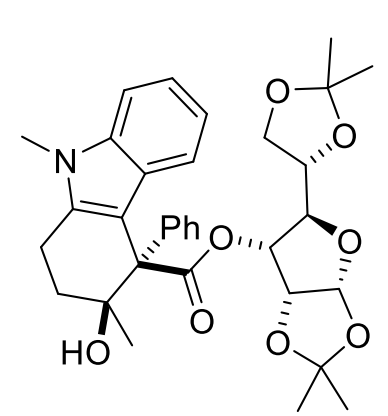

White solid, 62\% yield, ${ }^{1} \mathrm{H}$ NMR (400 MHz, Chloroform- $d$ ) $\delta$ $7.22-7.13(\mathrm{~m}, 6 \mathrm{H}), 7.06(\mathrm{t}, J=6.5 \mathrm{~Hz}, 1 \mathrm{H}), 6.88(\mathrm{~d}, J=7.6$ Hz, 1H), 6.83 (t, $J=6.6 \mathrm{~Hz}, 1 \mathrm{H}), 5.34(\mathrm{~s}, 1 \mathrm{H}), 5.03(\mathrm{~s}, 1 \mathrm{H})$, $3.94(\mathrm{~d}, J=9.0 \mathrm{~Hz}, 1 \mathrm{H}), 3.91-3.85(\mathrm{~m}, 2 \mathrm{H}), 3.80-3.72(\mathrm{~m}$, 1H), $3.62(\mathrm{~s}, 3 \mathrm{H}), 3.28(\mathrm{~s}, 1 \mathrm{H}), 2.90(\mathrm{~d}, J=13.5 \mathrm{~Hz}, 2 \mathrm{H}), 2.83$ $-2.75(\mathrm{~m}, 1 \mathrm{H}), 2.01-1.94(\mathrm{~m}, 1 \mathrm{H}), 1.60-1.52(\mathrm{~m}, 1 \mathrm{H}), 1.39$ (s, 6H), 1.30 (s, 3H), 0.88 (s, 3H), $0.68(\mathrm{~s}, 3 \mathrm{H}) .{ }^{13} \mathrm{C} \mathrm{NMR}\left(100 \mathrm{MHz}, \mathrm{CDCl}_{3}\right) \delta 173.2$, $136.7,136.7,136.0,129.2,128.9,126.0,125.7,125.1,121.9,120.0,117.7,110.8,108.4$, 107.5, 106.4, 104.2, 81.8, 79.3, 74.4, 73.6, 70.9, 66.4, 60.0, 34.9, 33.9, 30.9, 30.4, 29.2, $28.7,28.6,28.3,26.2,26.0,25.6,24.9,24.5,24.3,22.6,21.7,20.1,13.1,-0.0$. HRMS(ESI) Calcd. for $\mathrm{C}_{33} \mathrm{H}_{39} \mathrm{NO}_{8} \mathrm{Na}(\mathrm{M}+\mathrm{Na})^{+}$600.2688, found: 600.2679. 
a,5-dimethyl-9c-phenyl-3,4,5,9c-tetrahydrooxeto[2,3-c] carbazol-1(2aH)-one(5ab):

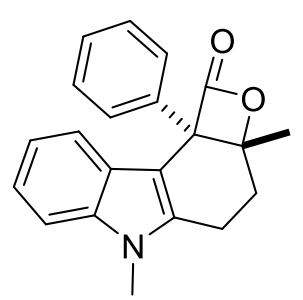

Yellow oil, $90 \%$ yield, ${ }^{1} \mathrm{H}$ NMR (400 MHz, Chloroform- $d$ ) $\delta 7.89(\mathrm{~d}$, $J=7.5 \mathrm{~Hz}, 1 \mathrm{H}), 7.53(\mathrm{t}, J=7.7 \mathrm{~Hz}, 1 \mathrm{H}), 7.41(\mathrm{~d}, J=7.5 \mathrm{~Hz}, 1 \mathrm{H}), 7.35$ -7.28 (m, 1H), $7.26-7.23(\mathrm{~m}, 1 \mathrm{H}), 7.09$ (t, $J=7.7 \mathrm{~Hz}, 1 \mathrm{H}), 6.99$ (d, $J=7.6 \mathrm{~Hz}, 1 \mathrm{H}), 6.79(\mathrm{t}, J=7.7 \mathrm{~Hz}, 1 \mathrm{H}), 6.39(\mathrm{~d}, J=8.0 \mathrm{~Hz}, 1 \mathrm{H}), 3.72$ (s, 3H), 3.08 (dd, $J=16.3,5.5 \mathrm{~Hz}, 1 \mathrm{H}), 2.85(\mathrm{ddd}, J=16.7,12.6,5.4 \mathrm{~Hz}, 1 \mathrm{H}), 2.57$ (dd, $J=15.1,5.3 \mathrm{~Hz}, 1 \mathrm{H}), 1.95(\mathrm{td}, J=13.7,5.5 \mathrm{~Hz}, 2 \mathrm{H}), 1.30(\mathrm{~s}, 3 \mathrm{H}) .{ }^{13} \mathrm{C}$ NMR $(100$ $\left.\mathrm{MHz}, \mathrm{CDCl}_{3}\right) \delta 169.8,137.7,136.1,136.0,129.1,128.5,128.0,125.9,121.3,119.6$, 108.9, 105.4, 84.8, 77.4, 77.2, 77.0, 76.7, 65.1, 31.2, 29.7, 29.3, 24.9, 17.7, 1.0. HRMS(ESI) Calcd. for $\mathrm{C}_{21} \mathrm{H}_{19} \mathrm{NO}_{2} \mathrm{Na}(\mathrm{M}+\mathrm{Na})^{+} 330.1409$, found: 330.1398 .

methyl 12-hydroxy-2-methoxy-6,12-dimethyl-11-phenyl-6H-5a,10b butanobenzofuro [2,3-b] indole-11-carboxylate $(5 \mathrm{ca})$ :<smiles>COc1ccc(C2(C(=O)O)CC3(C(=O)O)N(C)c4ccccc4C23C(C)=O)cc1</smiles>

Yellow oil, 42\% yield, ${ }^{1} \mathrm{H}$ NMR (400 MHz, Chloroform- $d$ ) $\delta 7.97$ (s, 1H), 7.55 (q, $J=7.5,7.0 \mathrm{~Hz}, 4 \mathrm{H}), 7.37(\mathrm{dq}, J=14.7,8.6,7.9 \mathrm{~Hz}$, $5 \mathrm{H}), 6.96-6.79(\mathrm{~m}, 3 \mathrm{H}), 6.63(\mathrm{~d}, J=8.8 \mathrm{~Hz}, 1 \mathrm{H}), 3.99(\mathrm{~s}, 3 \mathrm{H})$, $3.90(\mathrm{~s}, 3 \mathrm{H}), 3.75-3.72(\mathrm{~m}, 2 \mathrm{H}), 3.60-3.55(\mathrm{~m}, 2 \mathrm{H}), 2.42(\mathrm{~s}, 3 \mathrm{H})$, $1.57(\mathrm{~s}, 3 \mathrm{H}), 1.42(\mathrm{~s}, 1 \mathrm{H}) .{ }^{13} \mathrm{C} \mathrm{NMR}\left(100 \mathrm{MHz}, \mathrm{CDCl}_{3}\right) \delta 169.5$, $142.4,141.8,140.3,138.3,137.6,129.3,129.0,128.7,127.9,127.5,127.2$, 126.4, 122.8 , $118.87,118.5,117.4,111.3,109.9,108.3,57.3,55.8,52.1,48.9,31.4,30.2,29.7,29.2$, 22.3, 17.6. HRMS(ESI) Calcd. for $\mathrm{C}_{29} \mathrm{H}_{29} \mathrm{NO}_{5} \mathrm{Na}(\mathrm{M}+\mathrm{Na})^{+} 474.2079$, found: 474.2041.

methyl-3,9-dimethyl-4-phenyl-4,9-dihydro-1H-carbazole-4-carboxylate (5ba):

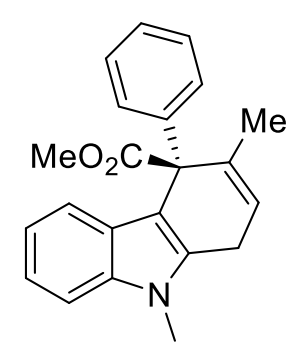
Yellow oil, 99\% yield, ${ }^{1} \mathrm{H}$ NMR (400 MHz, Chloroform- $d$ ) $\delta 7.30-$ $7.14(\mathrm{~m}, 8 \mathrm{H}), 7.14-7.08(\mathrm{~m}, 2 \mathrm{H}), 6.88(\mathrm{t}, J=7.5 \mathrm{~Hz}, 1 \mathrm{H}), 5.84(\mathrm{~s}$, $1 \mathrm{H}), 3.70(\mathrm{~s}, 3 \mathrm{H}), 3.68(\mathrm{~s}, 3 \mathrm{H}), 3.54-3.49(\mathrm{~m}, 2 \mathrm{H}), 1.68(\mathrm{~s}, 3 \mathrm{H}) .{ }^{13} \mathrm{C}$ NMR (101 MHz, $\left.\mathrm{CDCl}_{3}\right) \delta 173.1,141.8,137.4,135.9,134.6,128.8$, 
127.6, 126.3, 125.5, 121.1, 120.8, 119.3, 119.1, 109.4, 108.4, 58.2, 52.2, 29.3, 24.0, 21.1, 0.2. HRMS(ESI) Calcd.for $\mathrm{C}_{22} \mathrm{H}_{21} \mathrm{NO}_{2} \mathrm{Na}(\mathrm{M}+\mathrm{Na})^{+}$354.1469, found: 354.1470.

methyl 9-methyl-3-methylene-4-oxo-10-phenyl-1,2,3,4-tetrahydro-9H-4a,9a-

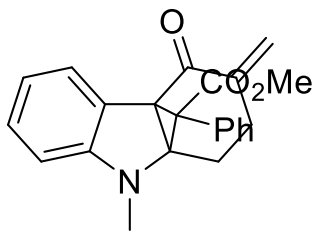
methanocarbazole-10-carboxylate (5de):

Yellow oil, 45\% yield, ${ }^{1} \mathrm{H}$ NMR (400 MHz, Chloroform- $d$ ) $\delta 7.35$ $(\mathrm{d}, J=8.0 \mathrm{~Hz}, 1 \mathrm{H}), 7.27-7.21(\mathrm{~m}, 2 \mathrm{H}), 7.20-7.08(\mathrm{~m}, 5 \mathrm{H}), 7.04$ (t, $J=7.6 \mathrm{~Hz}, 1 \mathrm{H}), 6.28(\mathrm{~s}, 1 \mathrm{H}), 5.51(\mathrm{~s}, 1 \mathrm{H}), 3.73(\mathrm{~s}, 6 \mathrm{H}), 3.21-3.06(\mathrm{~m}, 0 \mathrm{H}), 3.00-$ $2.87(\mathrm{~m}, 1 \mathrm{H}), 2.62-2.53(\mathrm{~m}, 1 \mathrm{H}), 2.47-2.35(\mathrm{~m}, 1 \mathrm{H}) .{ }^{13} \mathrm{C} \mathrm{NMR}\left(100 \mathrm{MHz}, \mathrm{CDCl}_{3}\right) \delta$ 195.2, 172.6, 146.0, 137.7, 136.8, 129.0, 128.2, 127.8, 127.3, 127.1, 121.6, 119.8, 119.2, 108.9, 105.5, 53.6, 52.7, 31.4, 30.2, 29.7, 29.0, 27.6. HRMS(ESI) Calcd. for $\mathrm{C}_{23} \mathrm{H}_{22} \mathrm{NO}_{3}(\mathrm{M}+\mathrm{H})^{+}$360.1566, found: 360.1600 .

9-methyl-3-methylene-1,2,3,9-tetrahydro-4H-carbazol-4-one (5dd): ${ }^{[\mathrm{e}]}$<smiles>C=C1CCc2c(c3ccccc3n2C)C1=O</smiles>
White solid,75\% yield, ${ }^{1} \mathrm{H}$ NMR (400 MHz, Chloroform- $d$ ) $\delta 8.29$ (d, $J=4.7 \mathrm{~Hz}, 1 \mathrm{H}), 7.33-7.15(\mathrm{~m}, 3 \mathrm{H}), 6.11(\mathrm{~s}, 1 \mathrm{H}), 5.34(\mathrm{~s}, 1 \mathrm{H}), 3.67-$ $3.51(\mathrm{~m}, 3 \mathrm{H}), 2.97-2.83(\mathrm{~m}, 4 \mathrm{H}) .{ }^{13} \mathrm{C} \mathrm{NMR}\left(100 \mathrm{MHz}, \mathrm{CDCl}_{3}\right) \delta$ $183.2,151.8,143.6,137.8,125.1,123.2,122.6,121.8,121.8,118.7$, $113.2,109.3,77.4,77.1,76.8,30.9,29.7,22.4$.

9-methyl-3-((2-methyl-1H-imidazol-1-yl)methyl)-1,2,3,9-tetrahydro-4H-carbazol4-one (5df): [f]

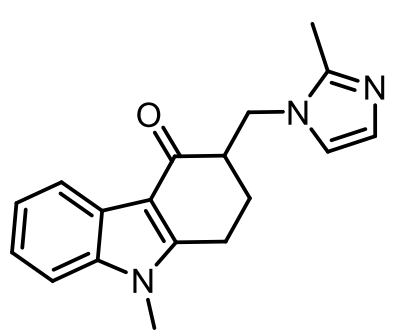

White solid, 99\% yield, ${ }^{1} \mathrm{H}$ NMR (400 MHz, Chloroform- $d$ ) $\delta 8.25(\mathrm{dd}, J=6.5,3.3 \mathrm{~Hz}, 1 \mathrm{H}), 7.37-7.28(\mathrm{~m}, 3 \mathrm{H}), 6.99-$ $6.85(\mathrm{~m}, 2 \mathrm{H}), 4.66(\mathrm{dd}, J=14.5,3.8 \mathrm{~Hz}, 1 \mathrm{H}), 4.15-4.00(\mathrm{~m}$, 1H), $3.67(\mathrm{~d}, J=3.6 \mathrm{~Hz}, 3 \mathrm{H}), 2.98(\mathrm{dt}, J=15.4,4.7 \mathrm{~Hz}, 1 \mathrm{H})$, $2.91-2.77(\mathrm{~m}, 2 \mathrm{H}), 2.43(\mathrm{~s}, 3 \mathrm{H}), 2.23-2.12(\mathrm{~m}, 1 \mathrm{H}), 1.93-$

$1.86(\mathrm{~m}, 1 \mathrm{H}) .{ }^{13} \mathrm{C} \mathrm{NMR}\left(100 \mathrm{MHz}, \mathrm{CDCl}_{3}\right) \delta 191.6,151.3,144.9,137.7,127.4,124.7$, 
123.4, 122.9, 121.5, 119.8, 112.2, 109.3, 77.4, 77.0, 76.7, 47.3, 45.7, 29.9, 26.6, 21.5, 13.3.

[e]

Kim, M. Y.; Lim, G. J.; Lim, J. I.; Kim, D. S.; Kim, I. Y.; Yang, J. S.

Heterocycles 1997, 45, 2041.

[f]

Visser, M.; Zaya, M. J.; Locuson, C. W.; Boothe, D. M.; Merritt, D.A. Xenobiotica 2018, 1-10. 


\section{NMR Spectra of Compounds}

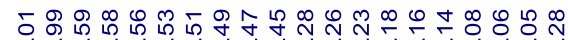

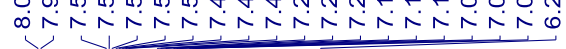

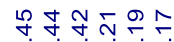

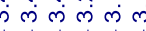
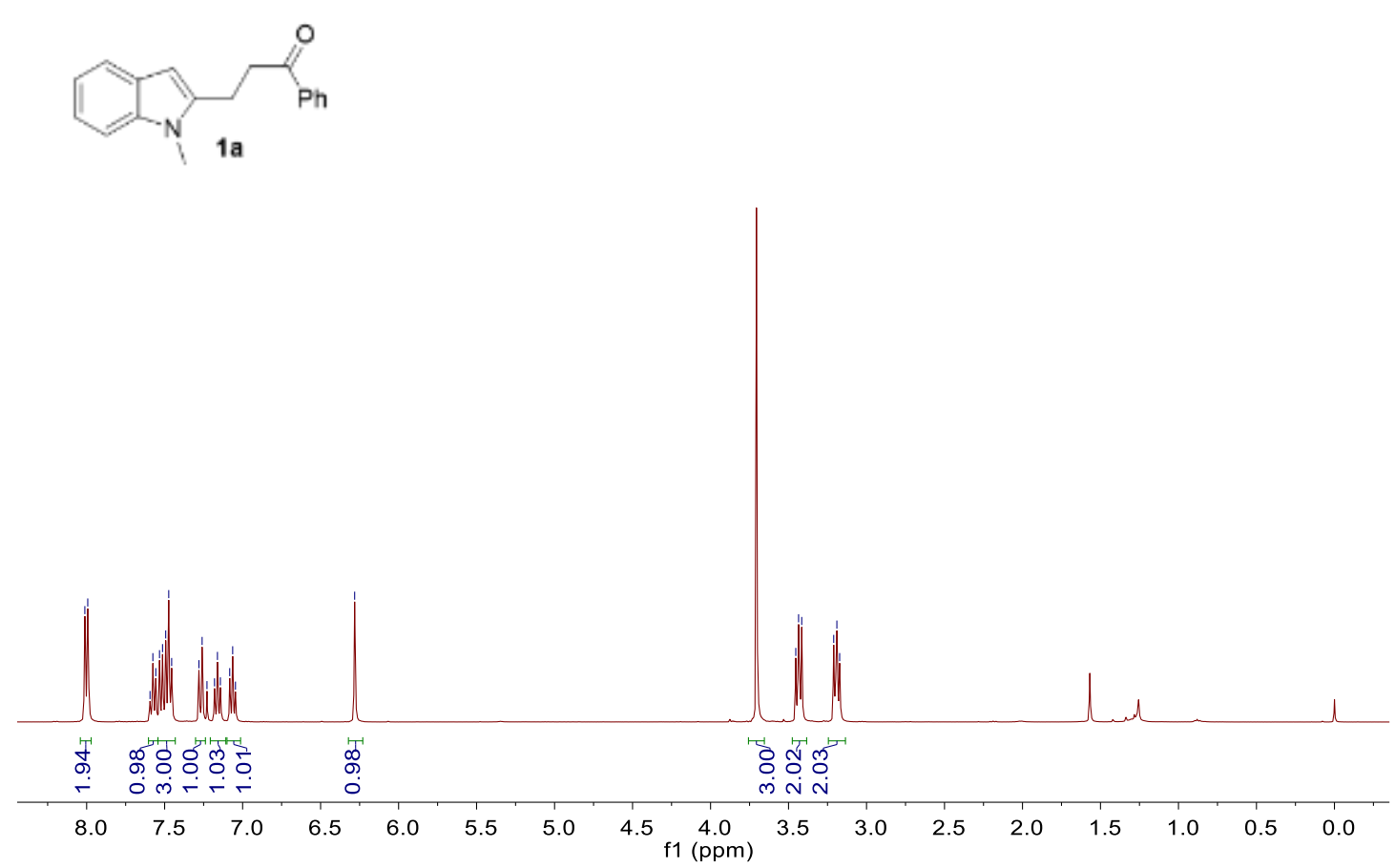

${ }^{1} \mathrm{H}$ NMR Spectrum of Compound $1 \mathrm{a}\left(400 \mathrm{MHz}, \mathrm{CDCl}_{3}\right)$.

\begin{tabular}{l}
0 \\
$\stackrel{\infty}{\infty}$ \\
$\stackrel{\infty}{\leftarrow}$ \\
\hline
\end{tabular}
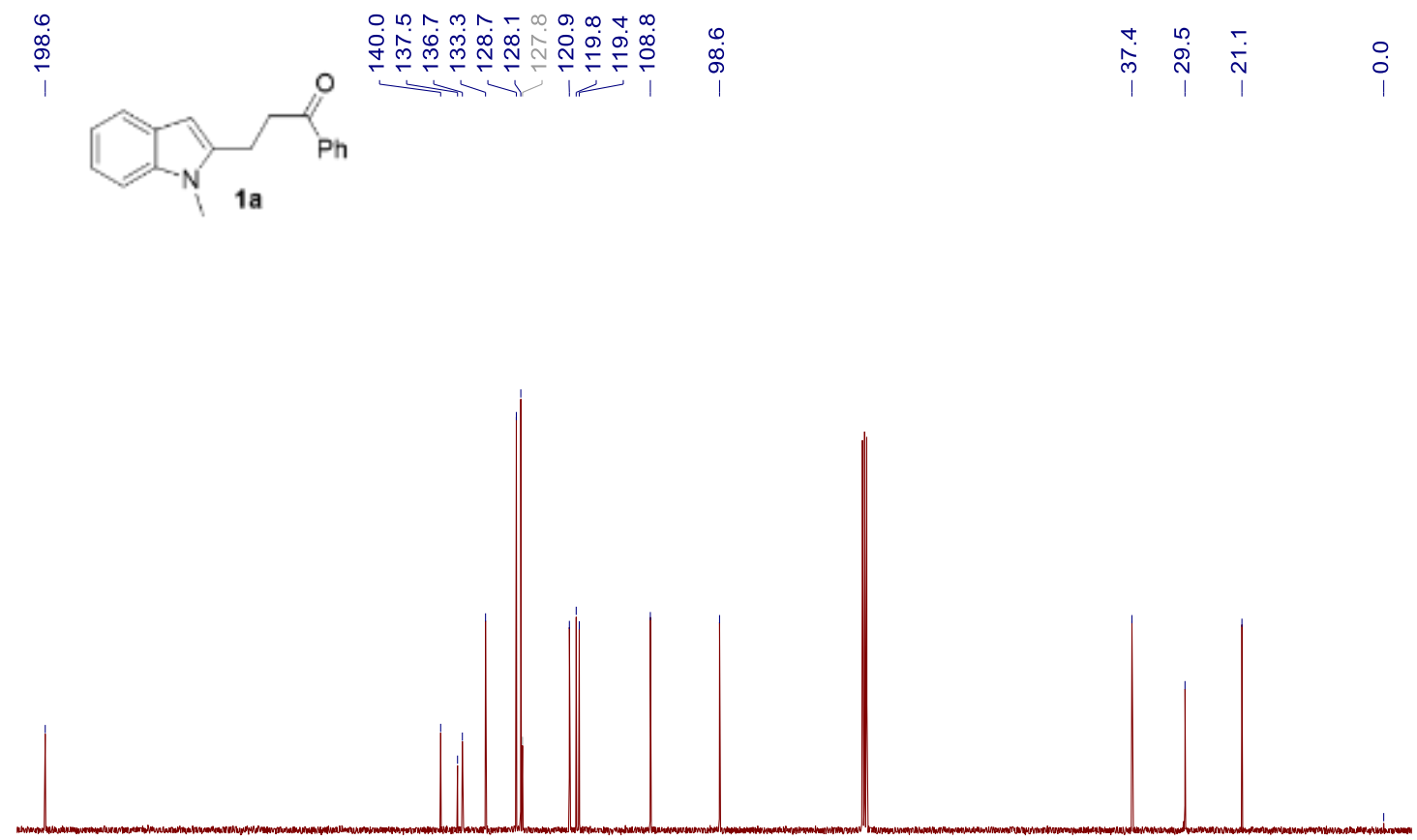

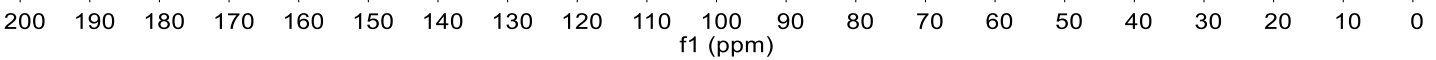

${ }^{13} \mathrm{C}$ NMR Spectrum of Compound $1 \mathbf{a}\left(100 \mathrm{MHz}, \mathrm{CDCl}_{3}\right)$. 


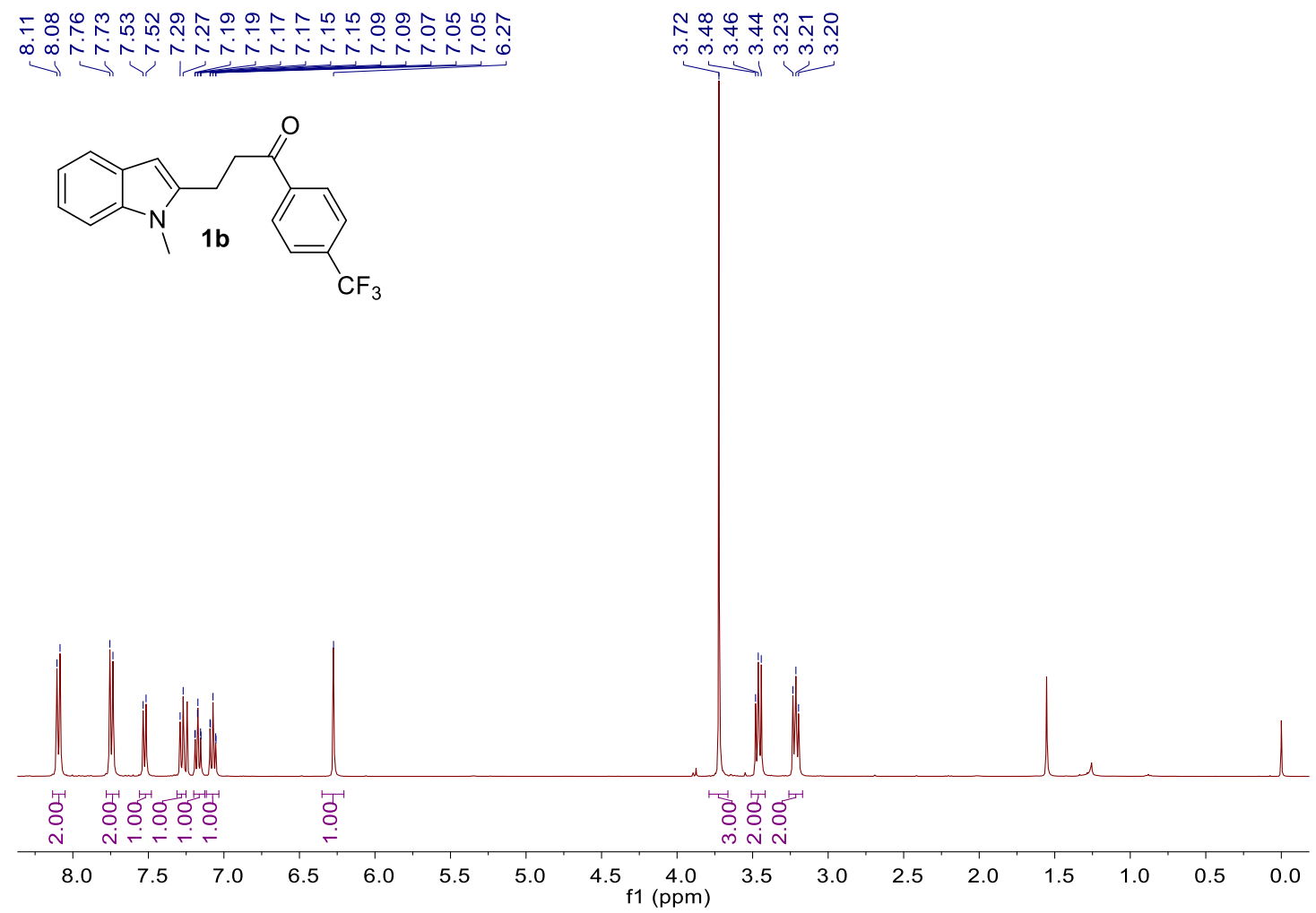

${ }^{1} \mathrm{H}$ NMR Spectrum of Compound $\mathbf{1 b}\left(400 \mathrm{MHz}, \mathrm{CDCl}_{3}\right)$.

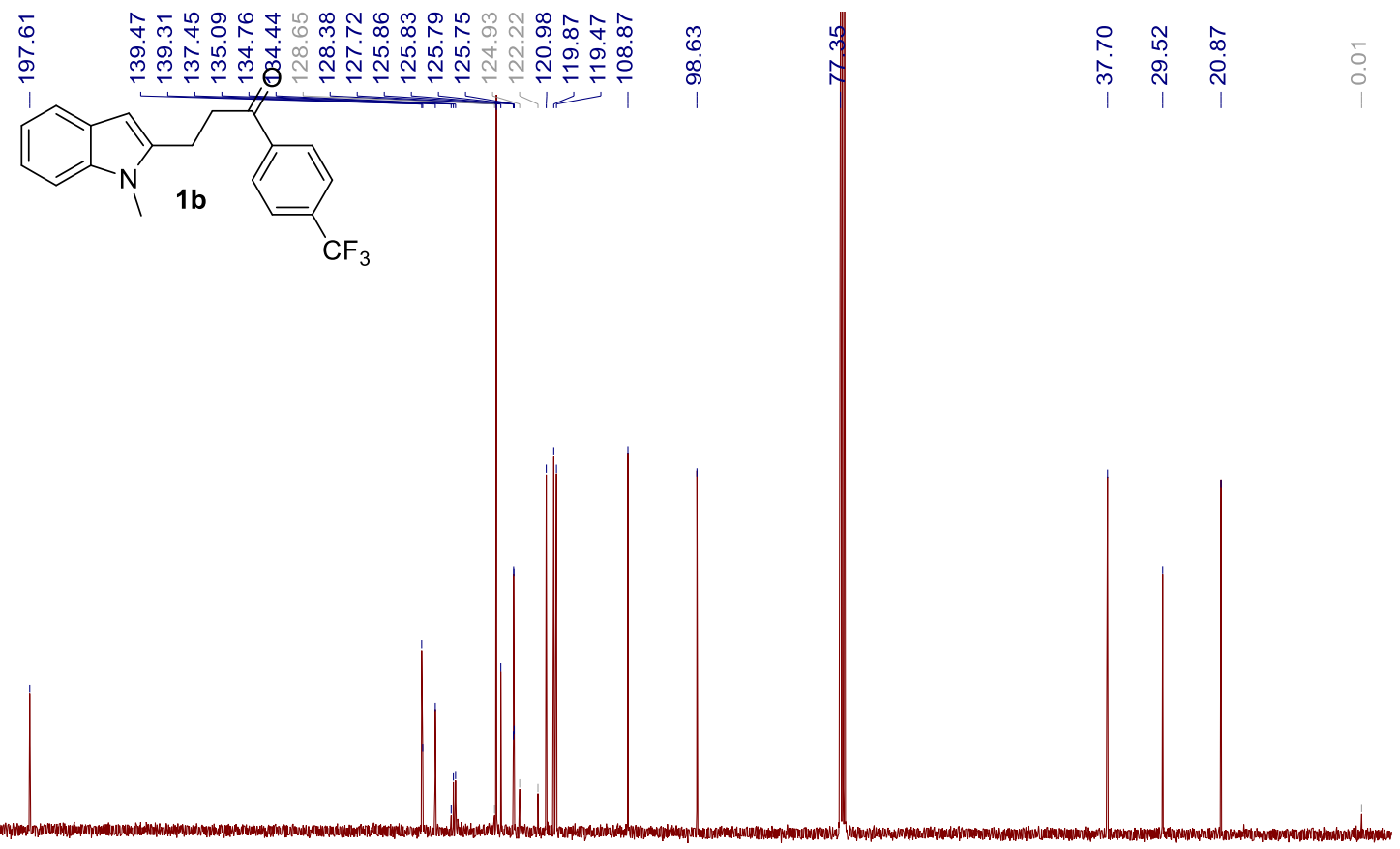

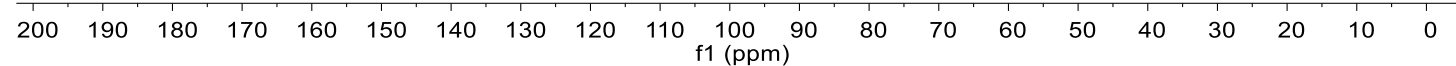

${ }^{13} \mathrm{C}$ NMR Spectrum of Compound $\mathbf{1 b}\left(100 \mathrm{MHz}, \mathrm{CDCl}_{3}\right)$. 

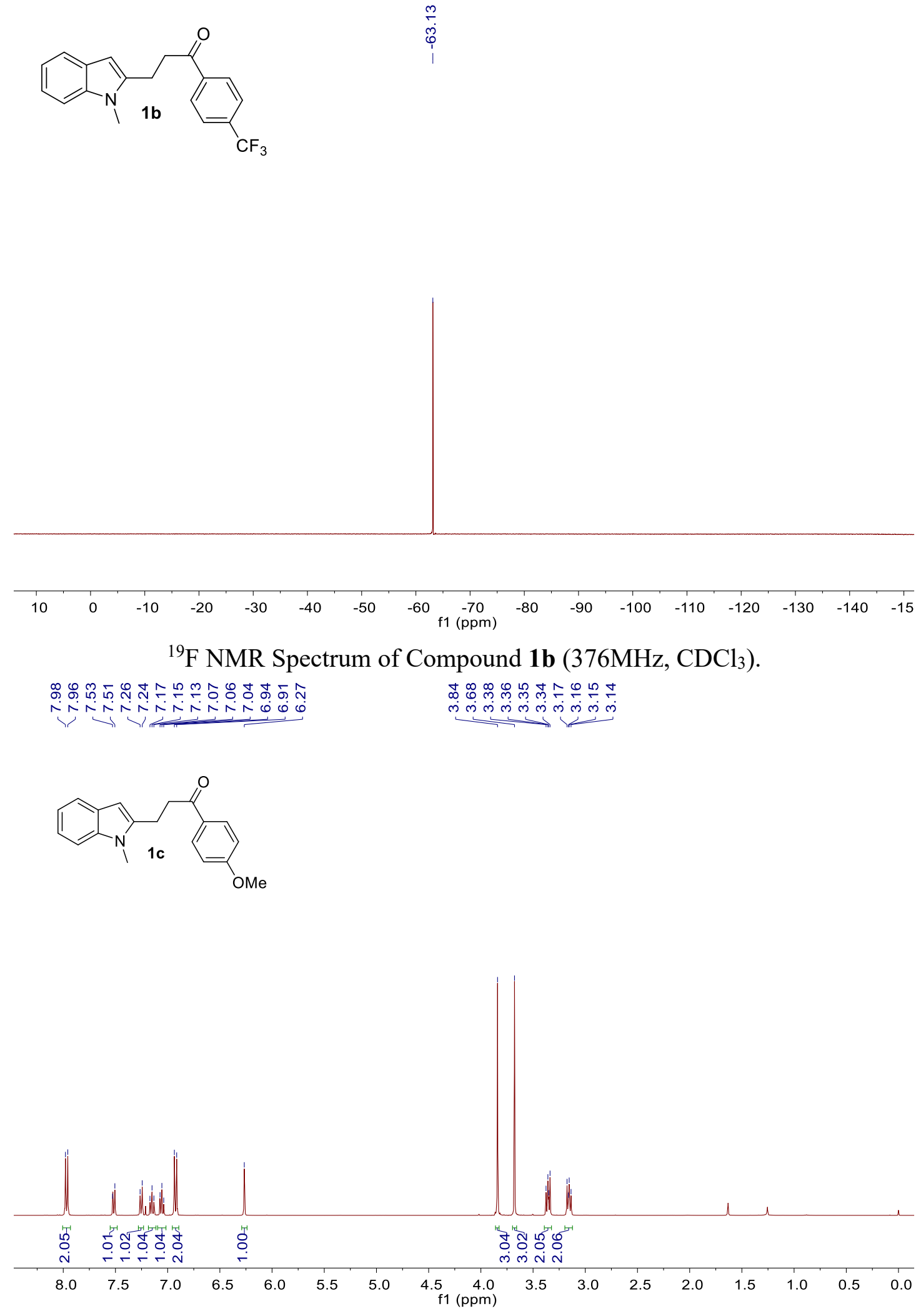

${ }^{1} \mathrm{H}$ NMR Spectrum of Compound $1 \mathrm{c}\left(400 \mathrm{MHz}, \mathrm{CDCl}_{3}\right)$. 


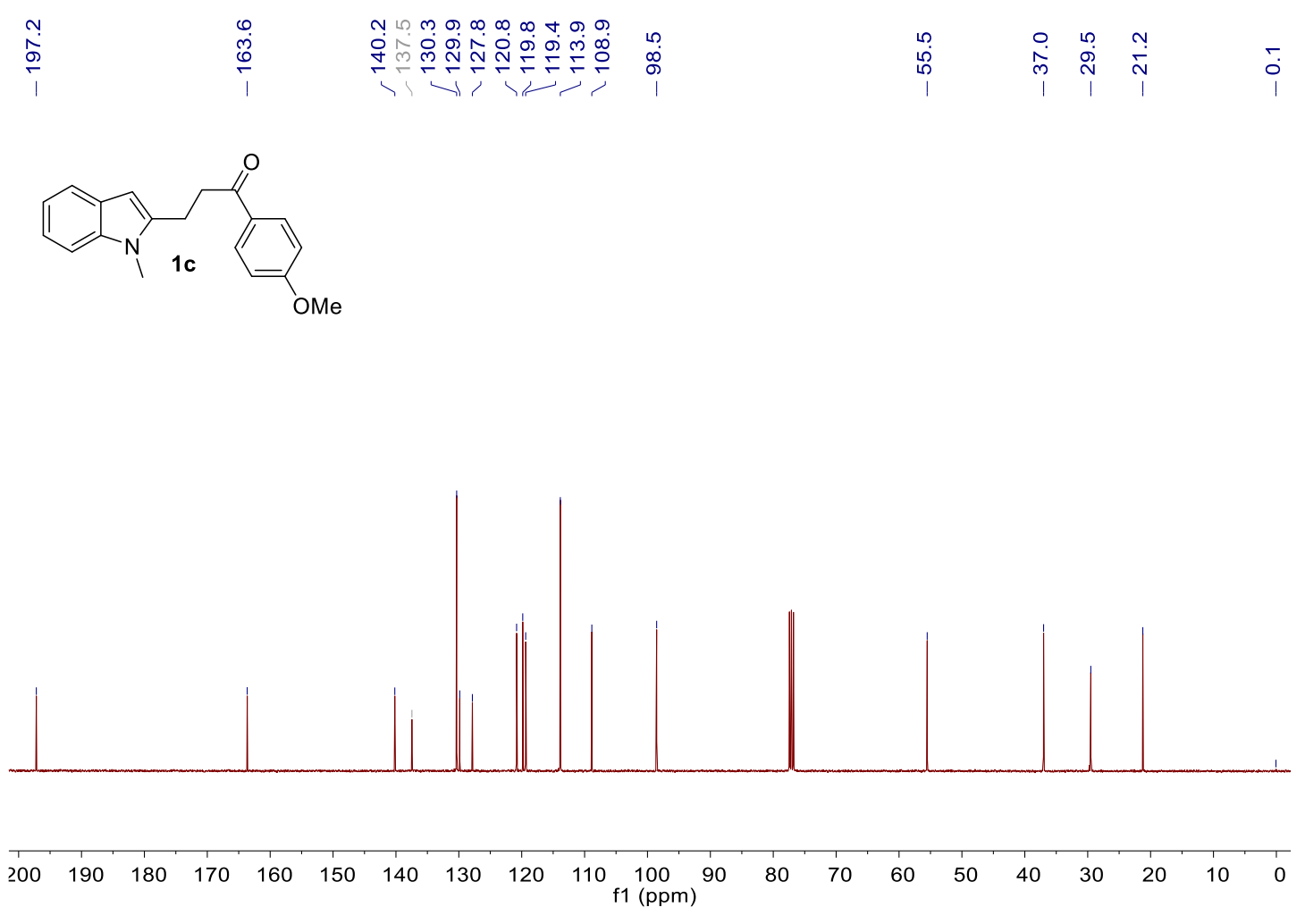

${ }^{13} \mathrm{C}$ NMR Spectrum of Compound $1 \mathrm{c}\left(100 \mathrm{MHz}, \mathrm{CDCl}_{3}\right)$.

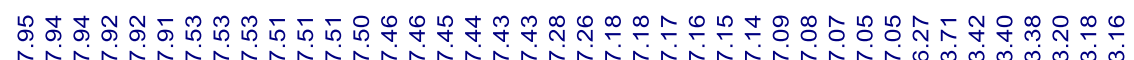

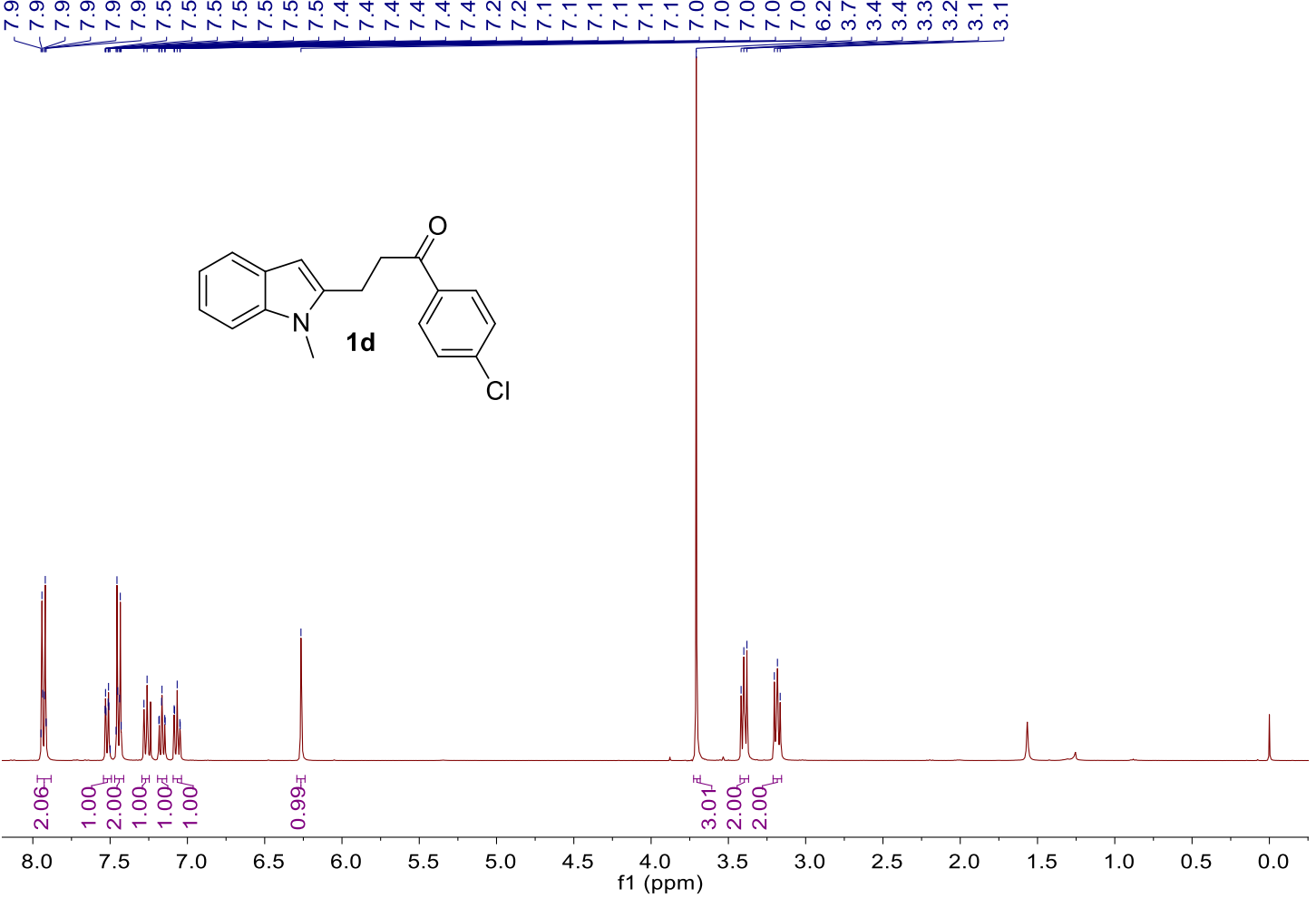

${ }^{1} \mathrm{H}$ NMR Spectrum of Compound $\mathbf{1 d}\left(400 \mathrm{MHz}, \mathrm{CDCl}_{3}\right)$. 

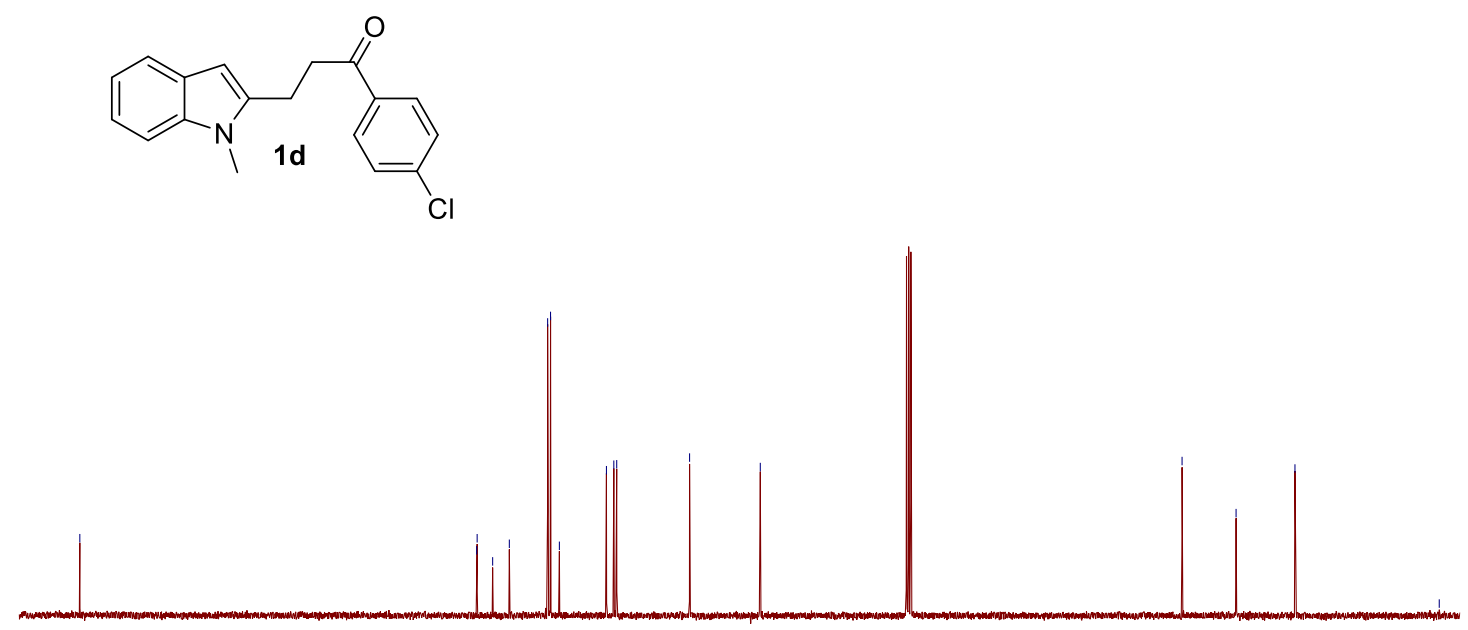

$\begin{array}{lllllllllllllllllllll}200 & 190 & 180 & 170 & 160 & 150 & 140 & 130 & 120 & \begin{array}{c}110 \\ \mathrm{f} 1(\mathrm{ppm})\end{array} & 90 & 80 & 70 & 60 & 50 & 40 & 30 & 20 & 10 & 0\end{array}$ ${ }^{13} \mathrm{C}$ NMR Spectrum of Compound 1d $\left(100 \mathrm{MHz}, \mathrm{CDCl}_{3}\right)$.
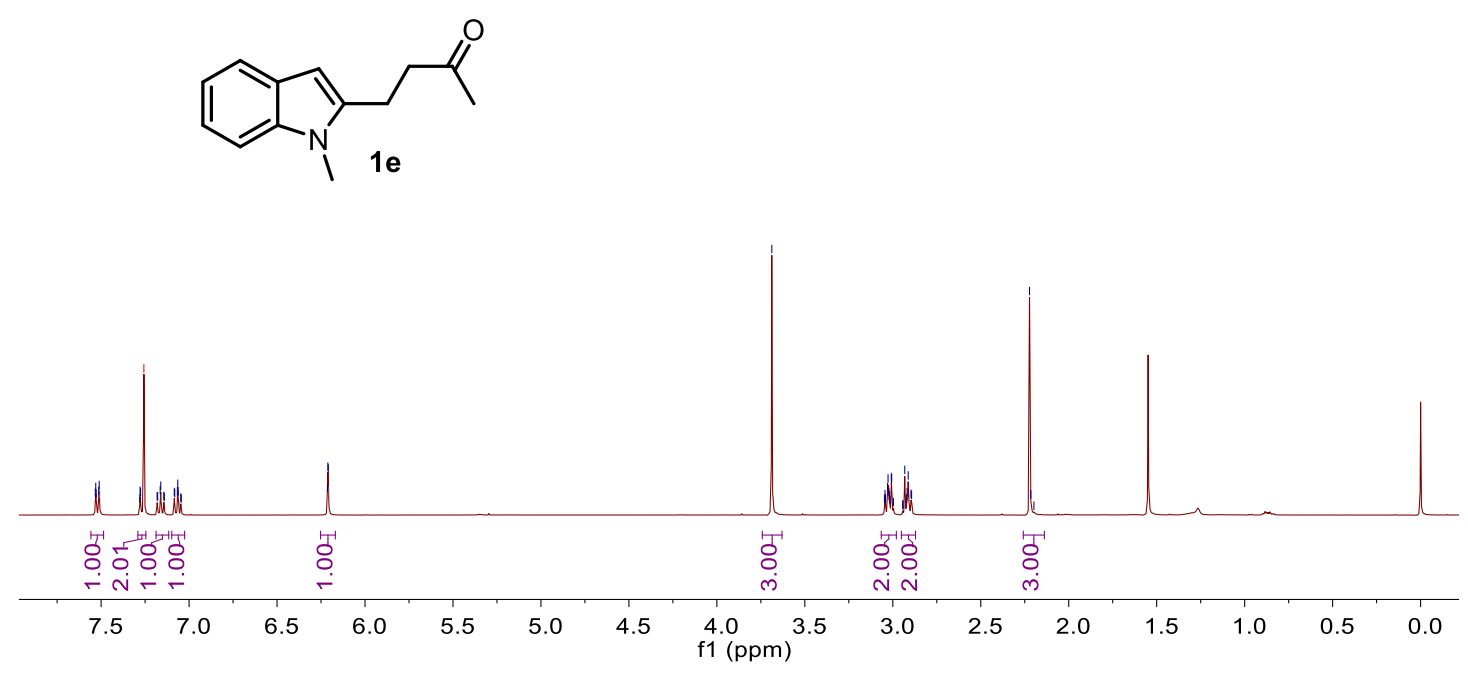

${ }^{1} \mathrm{H}$ NMR Spectrum of Compound $1 \mathrm{e}\left(400 \mathrm{MHz}, \mathrm{CDCl}_{3}\right)$. 

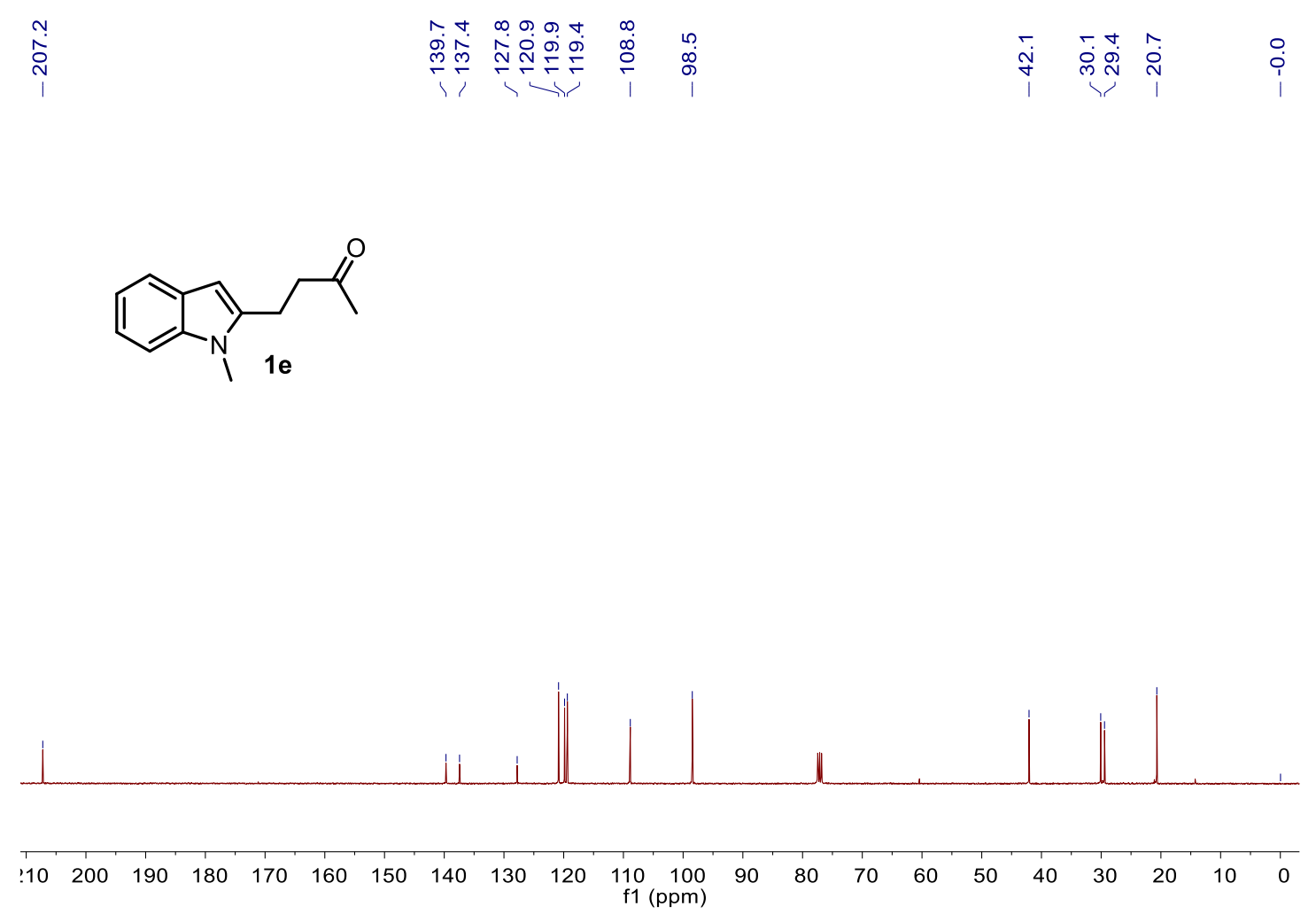

${ }^{13} \mathrm{C}$ NMR Spectrum of Compound $1 \mathrm{e}\left(100 \mathrm{MHz}, \mathrm{CDCl}_{3}\right)$.
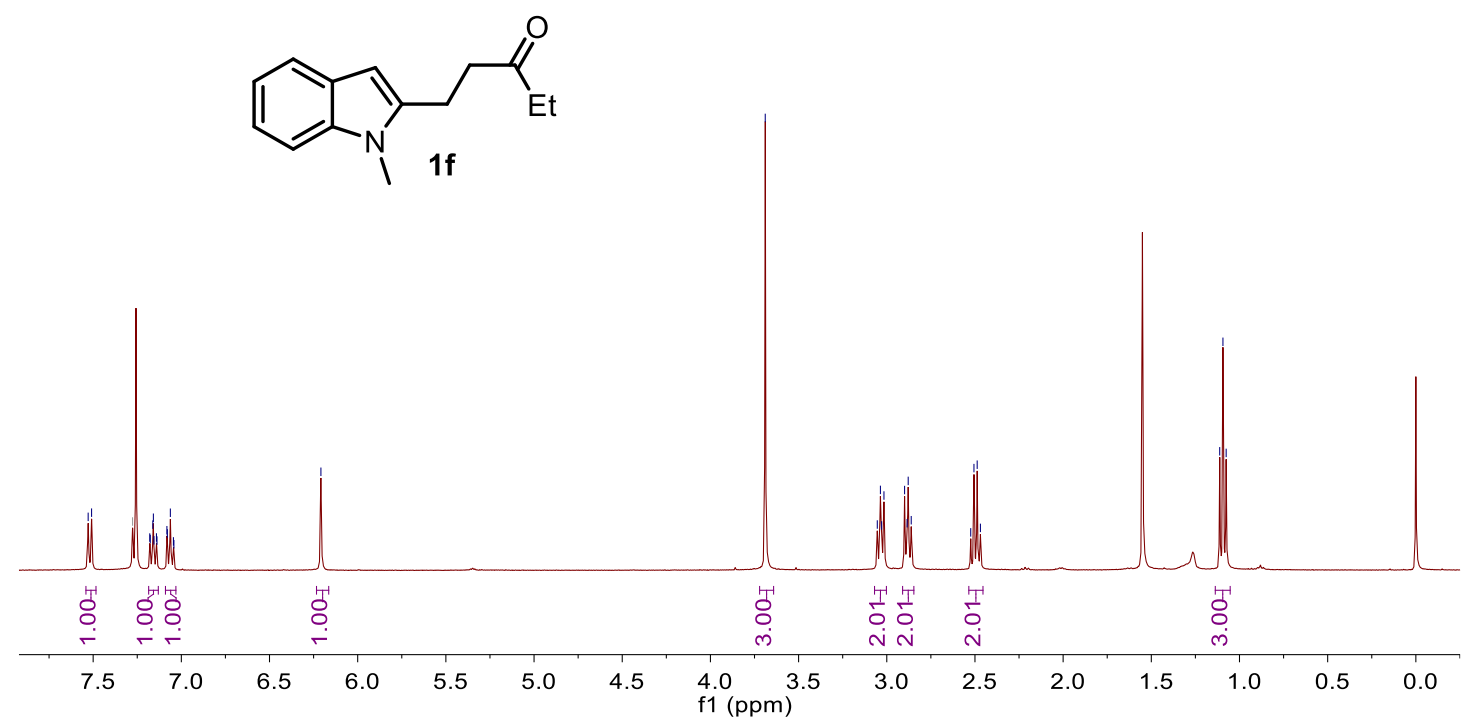

${ }^{1} \mathrm{H}$ NMR Spectrum of Compound $1 \mathbf{f}\left(400 \mathrm{MHz}, \mathrm{CDCl}_{3}\right)$. 

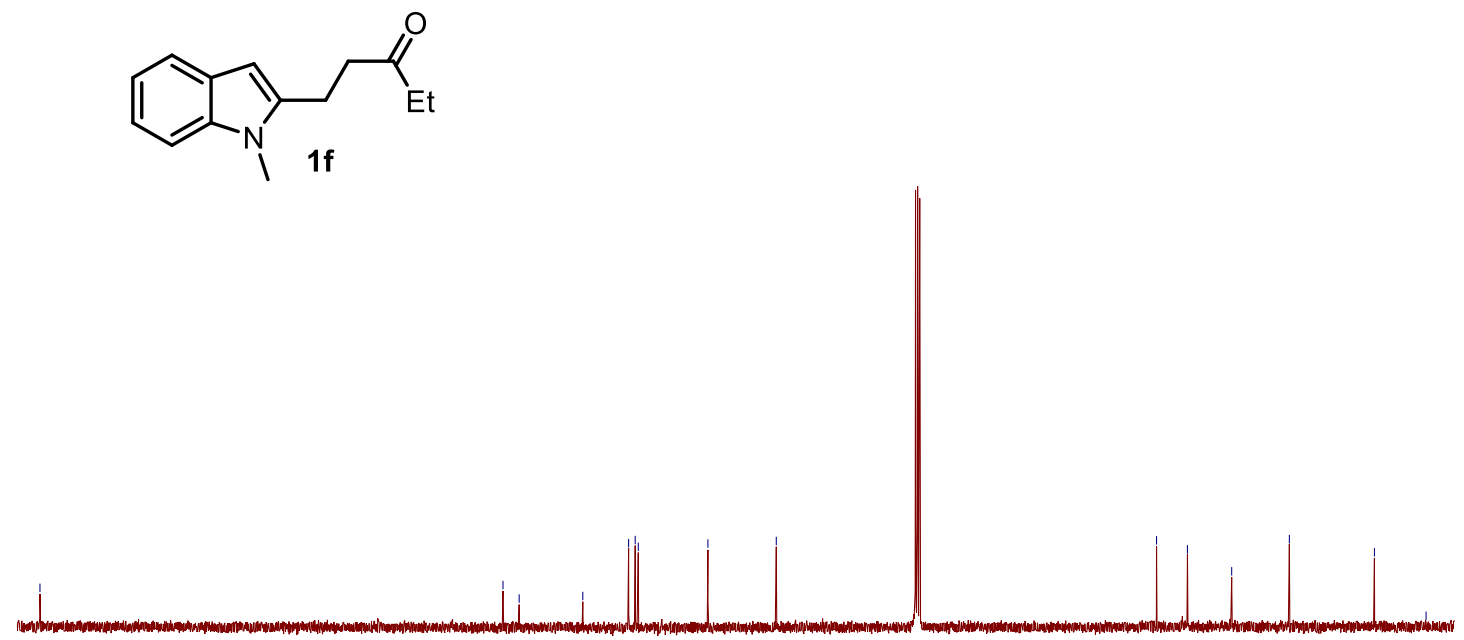

$\begin{array}{llllllllllllllllllllll}210 & 200 & 190 & 180 & 170 & 160 & 150 & 140 & 130 & 120 & \begin{array}{c}110 \\ \mathrm{f} 1(\mathrm{ppm})\end{array} & 90 & 80 & 70 & 60 & 50 & 40 & 30 & 20 & 10 & 0\end{array}$

${ }^{13} \mathrm{C}$ NMR Spectrum of Compound $\mathbf{1 f}\left(100 \mathrm{MHz}, \mathrm{CDCl}_{3}\right)$.

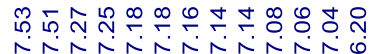

invinisingon

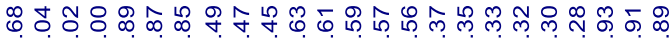<smiles>Cn1c(CCC(=O)Br)cc2ccccc21</smiles>

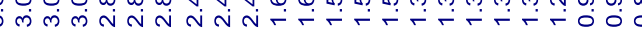

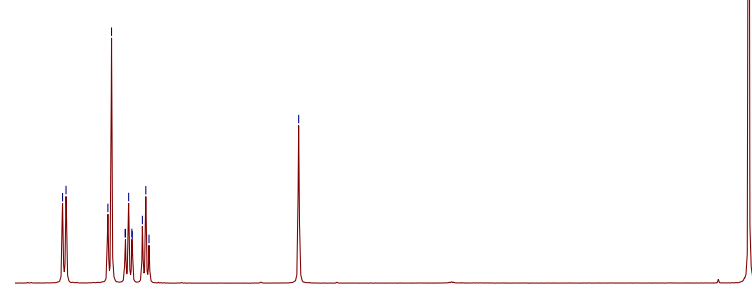

.

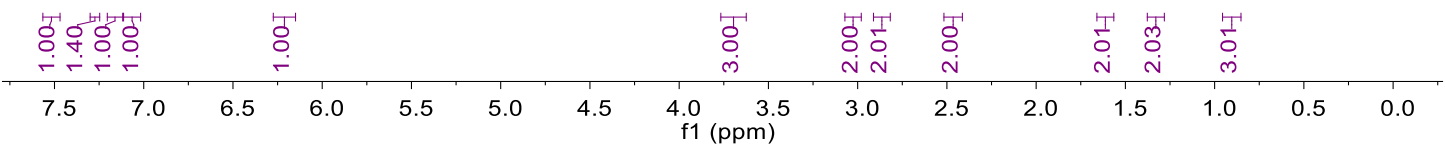

${ }^{1} \mathrm{H}$ NMR Spectrum of Compound $\mathbf{1 g}\left(400 \mathrm{MHz}, \mathrm{CDCl}_{3}\right)$. 
i̊.

穴芯

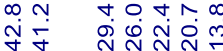<smiles>Cn1c(CCC(=O)Br)cc2ccccc21</smiles>

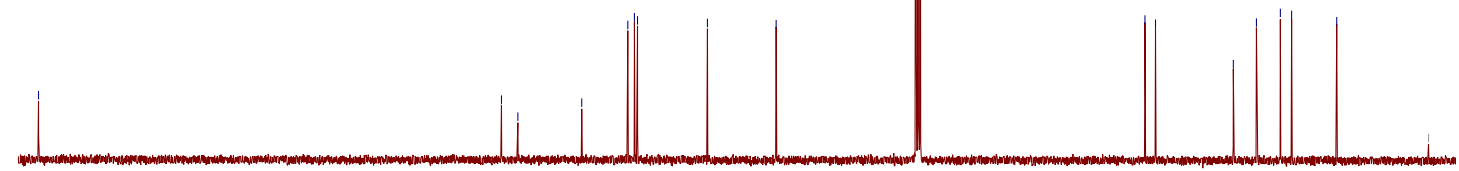

$\begin{array}{llllllllllllllllllllll}210 & 200 & 190 & 180 & 170 & 160 & 150 & 140 & 130 & 120 & \begin{array}{c}110 \\ \mathrm{f} 1(\mathrm{ppm})\end{array} & 90 & 80 & 70 & 60 & 50 & 40 & 30 & 20 & 10 & 0\end{array}$

${ }^{13} \mathrm{C}$ NMR Spectrum of Compound 1g (100MHz, $\left.\mathrm{CDCl}_{3}\right)$.
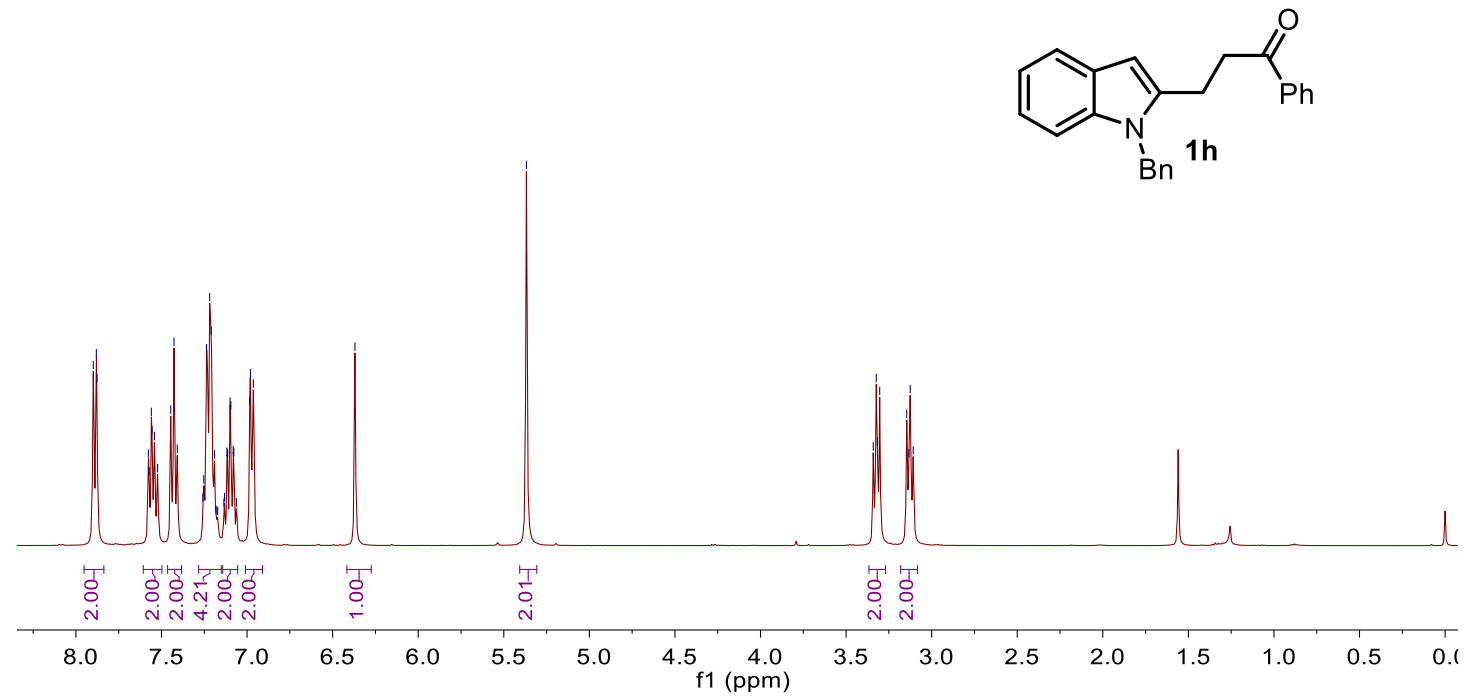

${ }^{1} \mathrm{H}$ NMR Spectrum of Compound $\mathbf{1 h}\left(400 \mathrm{MHz}, \mathrm{CDCl}_{3}\right)$. 

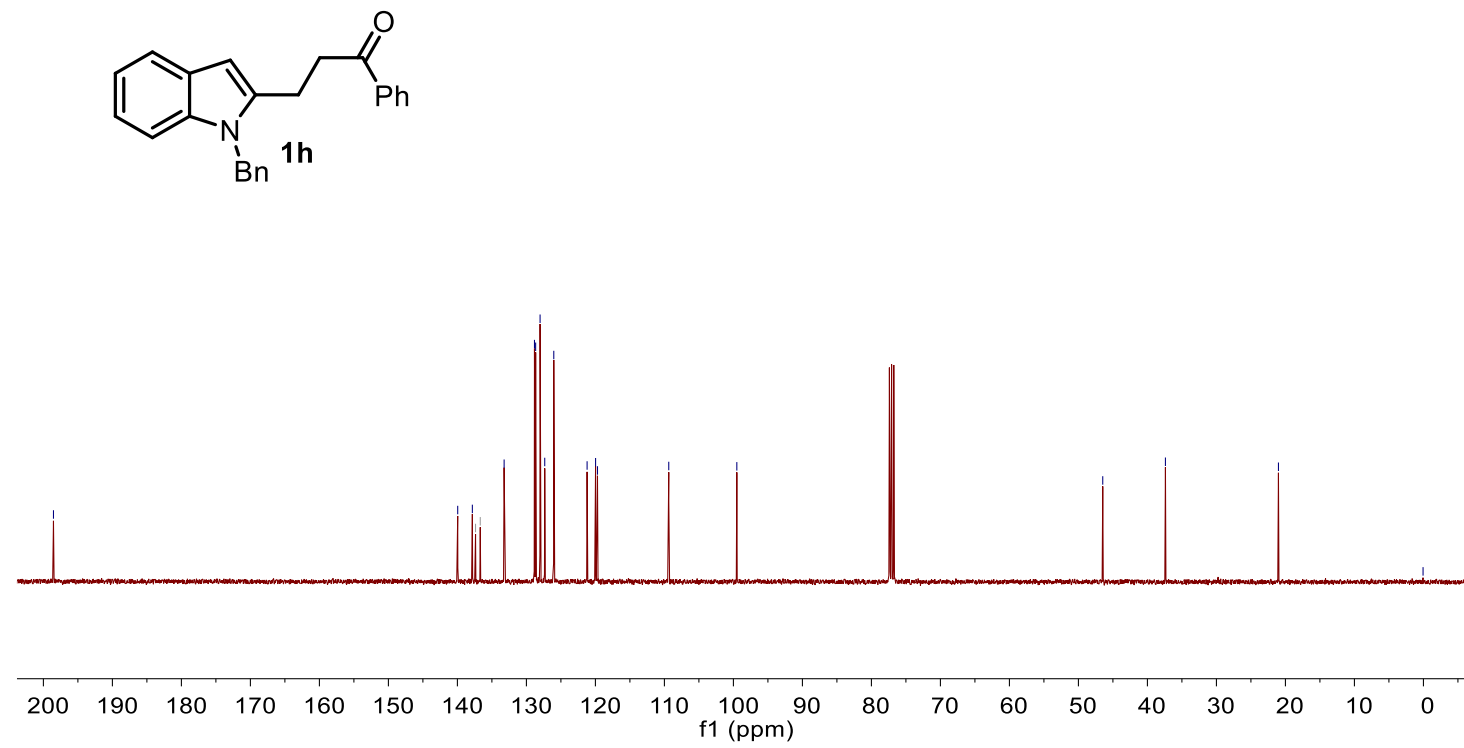

${ }^{13} \mathrm{C}$ NMR Spectrum of Compound $\mathbf{1 h}\left(100 \mathrm{MHz}, \mathrm{CDCl}_{3}\right)$.

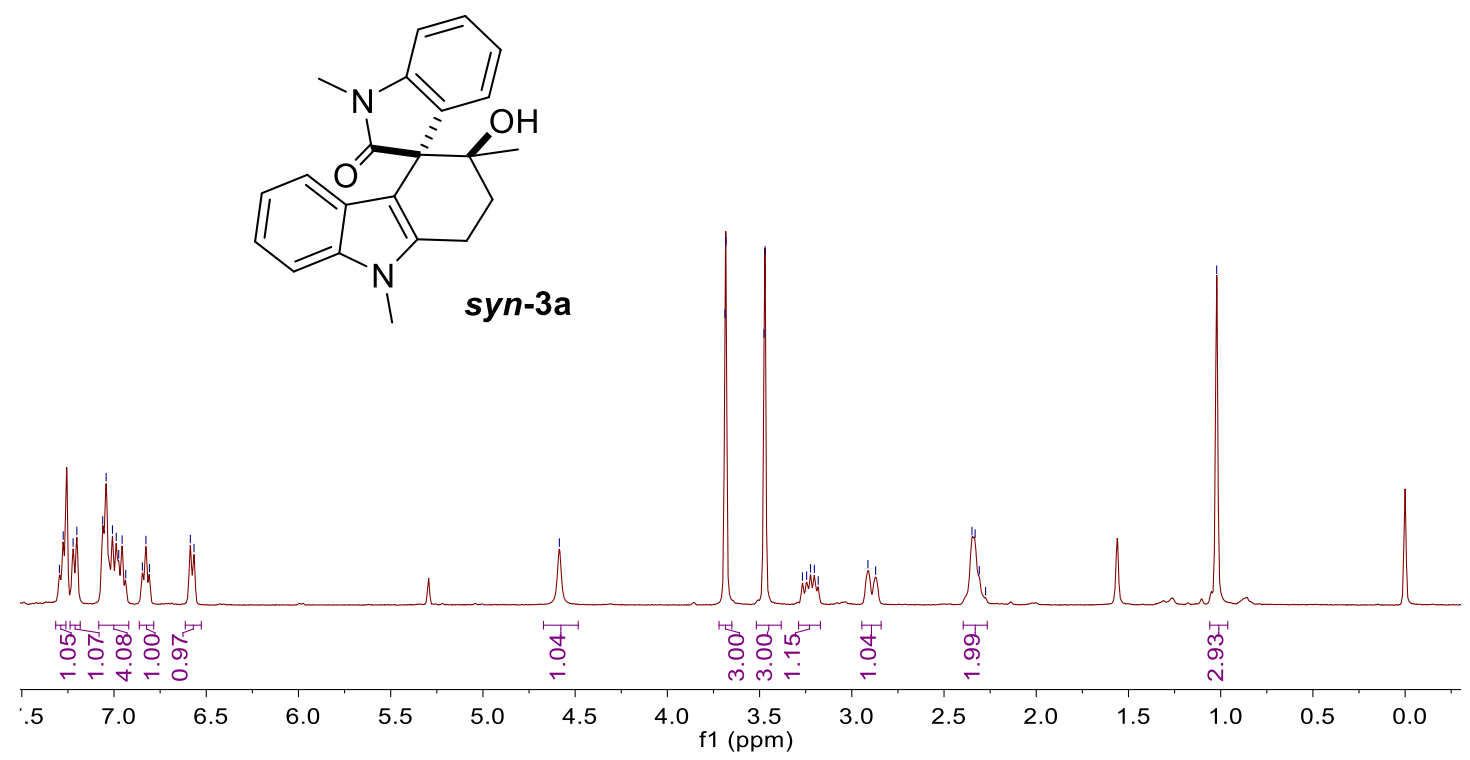

${ }^{1} \mathrm{H}$ NMR Spectrum of Compound 3a $\left(400 \mathrm{MHz}, \mathrm{CDCl}_{3}\right)$. 


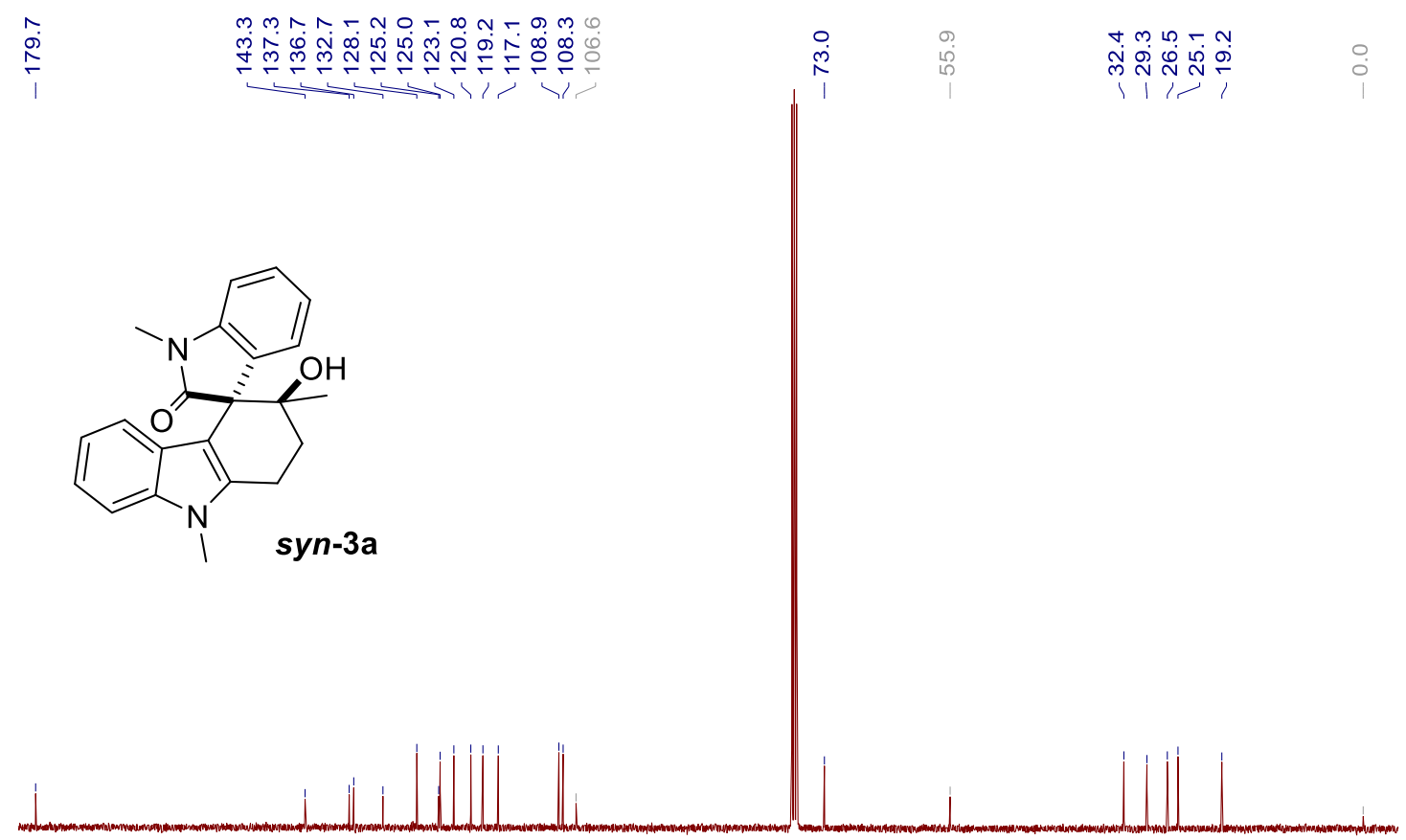

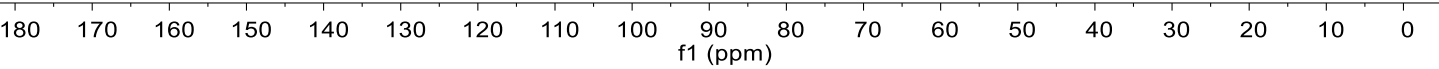

${ }^{13} \mathrm{C}$ NMR Spectrum of Compound 3a $\left(100 \mathrm{MHz}, \mathrm{CDCl}_{3}\right)$.
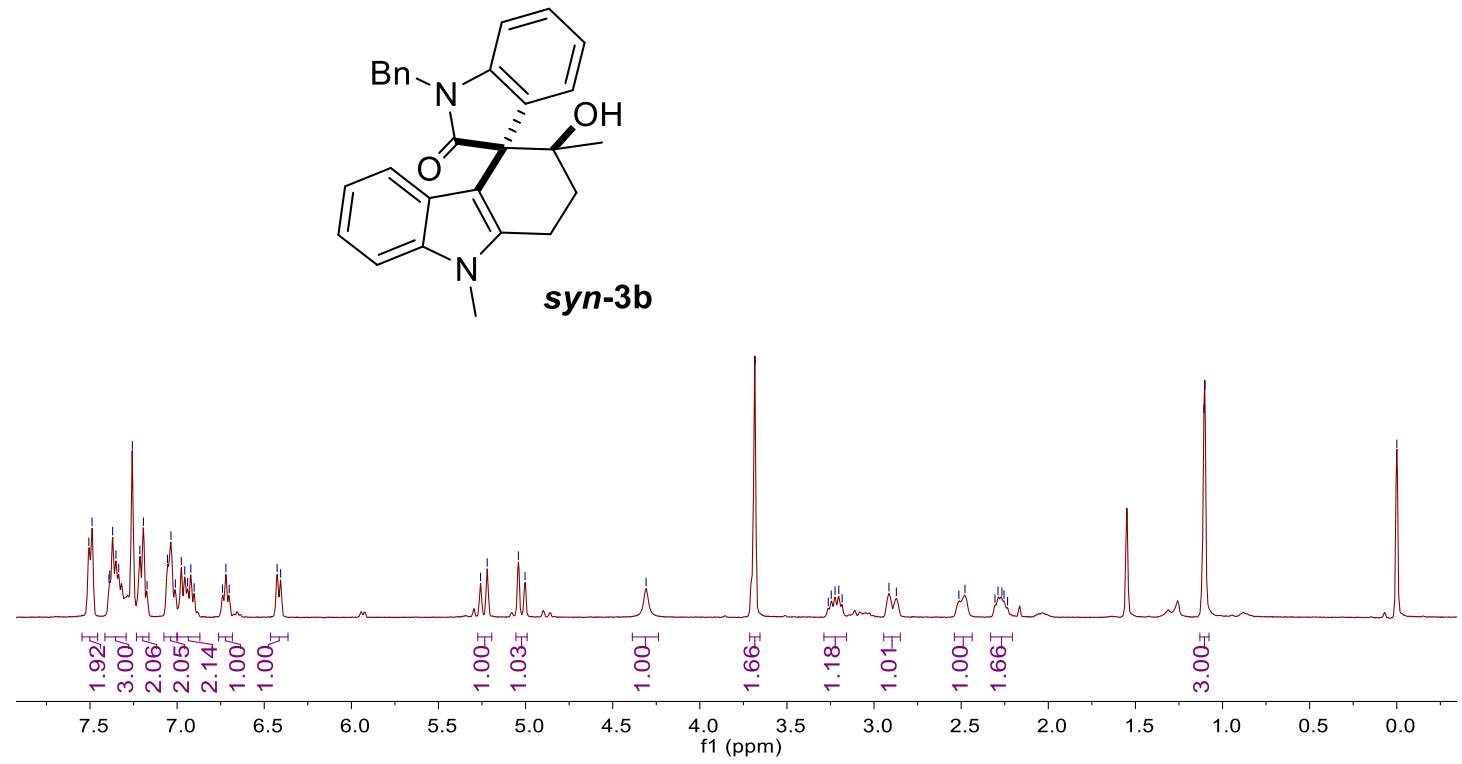

${ }^{1} \mathrm{H}$ NMR Spectrum of Compound $\mathbf{3 b}\left(400 \mathrm{MHz}, \mathrm{CDCl}_{3}\right)$. 

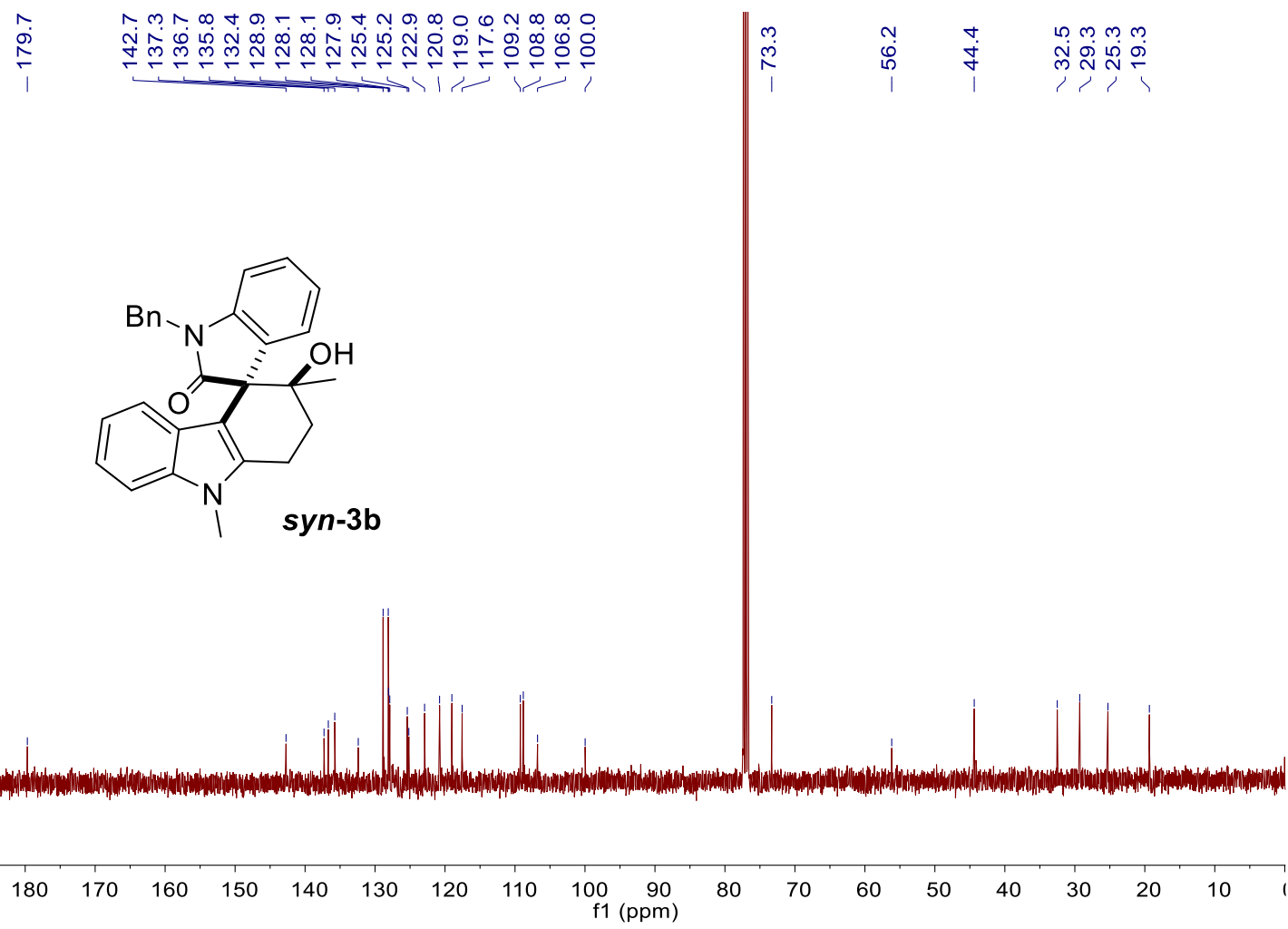

${ }^{13} \mathrm{C}$ NMR Spectrum of Compound $\mathbf{3 b}\left(100 \mathrm{MHz}, \mathrm{CDCl}_{3}\right)$.

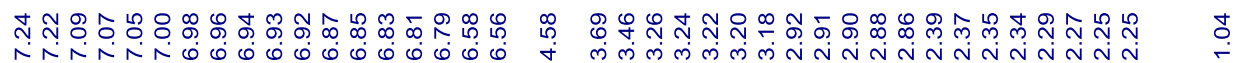
în<smiles>CN1C(=O)[C@]2(c3cc(F)ccc31)c1c(n(C)c3ccccc13)CCC2(C)O</smiles>

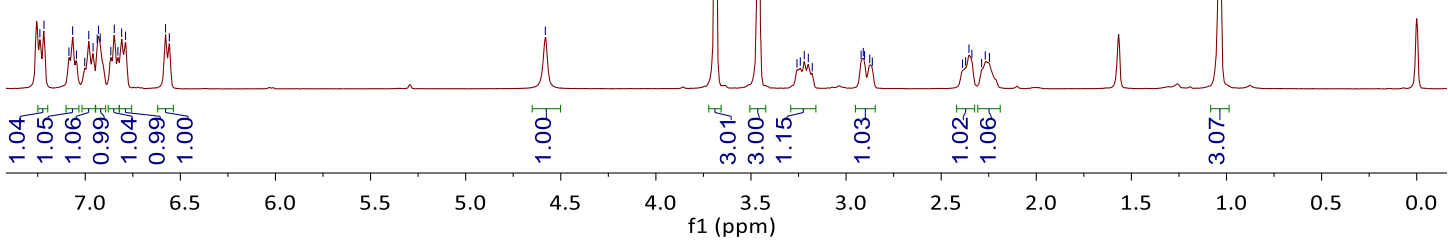

${ }^{1} \mathrm{H}$ NMR Spectrum of Compound $\mathbf{3 c}\left(400 \mathrm{MHz}, \mathrm{CDCl}_{3}\right)$. 

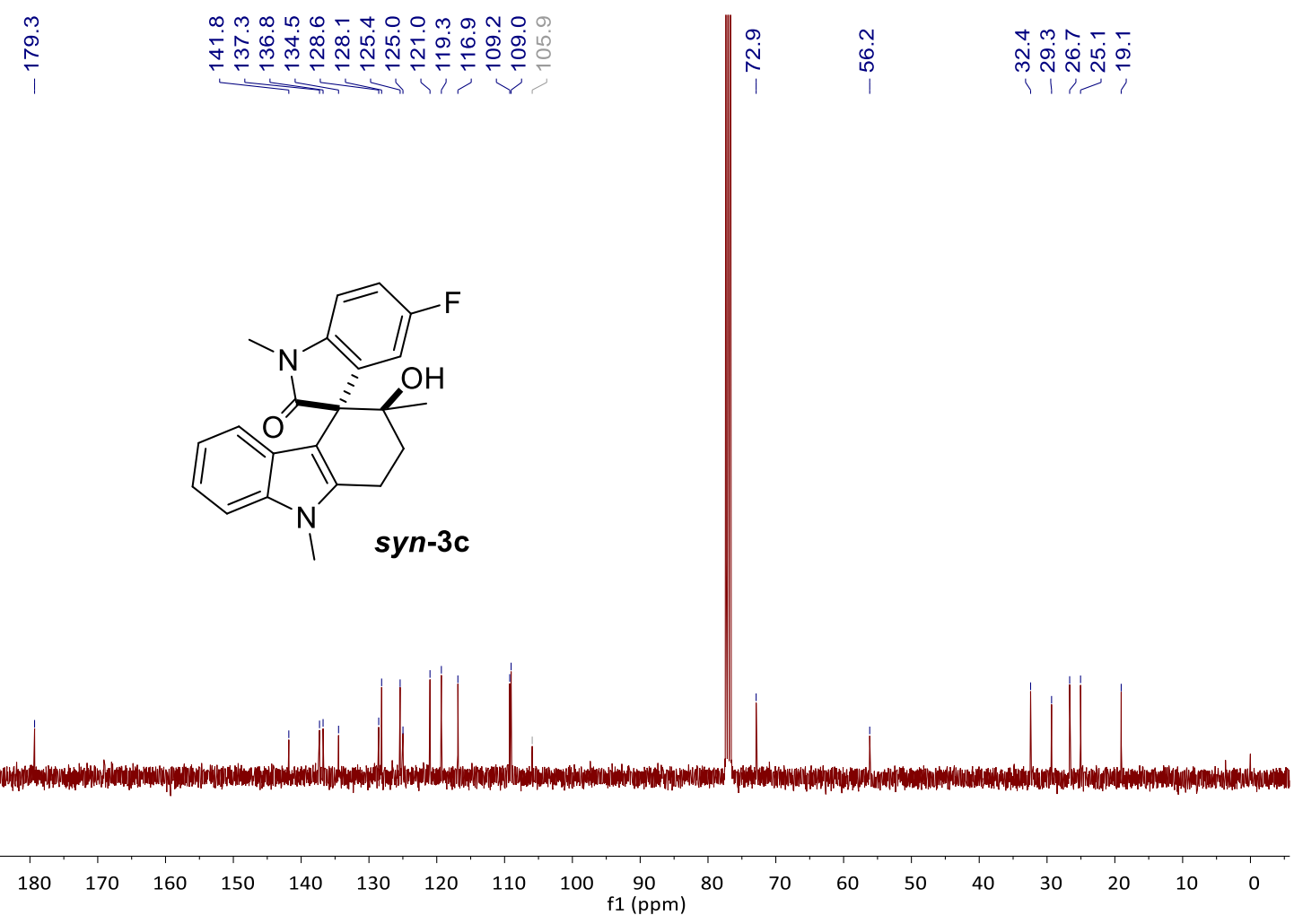

${ }^{13} \mathrm{C}$ NMR Spectrum of Compound $3 \mathbf{c}\left(100 \mathrm{MHz}, \mathrm{CDCl}_{3}\right)$.

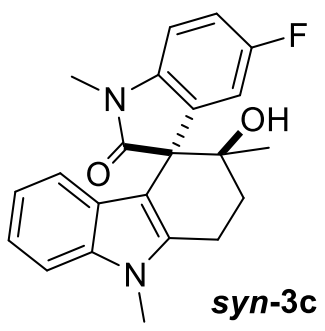

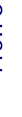

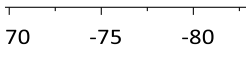

$\begin{array}{lll}-85 & -90 \quad-95\end{array}$

$\begin{array}{rr}-105 & -110 \\ & f 1(p p m)\end{array}$

$-115-120$

$\begin{array}{lllll}-125 & -130 & -135 & -140 & -145\end{array}$

${ }^{19} \mathrm{~F}$ NMR Spectrum of Compound $\mathbf{3 c}\left(376 \mathrm{MHz}, \mathrm{CDCl}_{3}\right)$. 

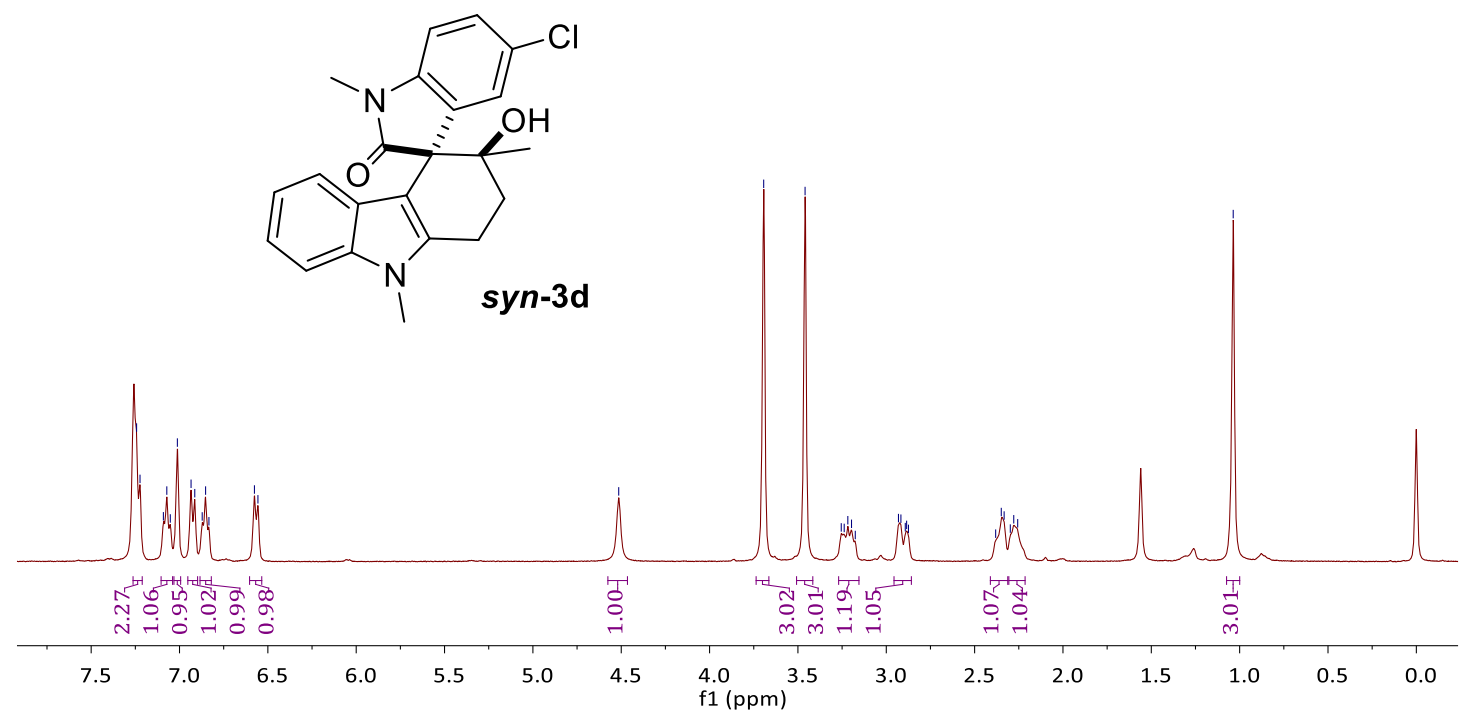

${ }^{1} \mathrm{H}$ NMR Spectrum of Compound 3d $\left(400 \mathrm{MHz}, \mathrm{CDCl}_{3}\right)$.
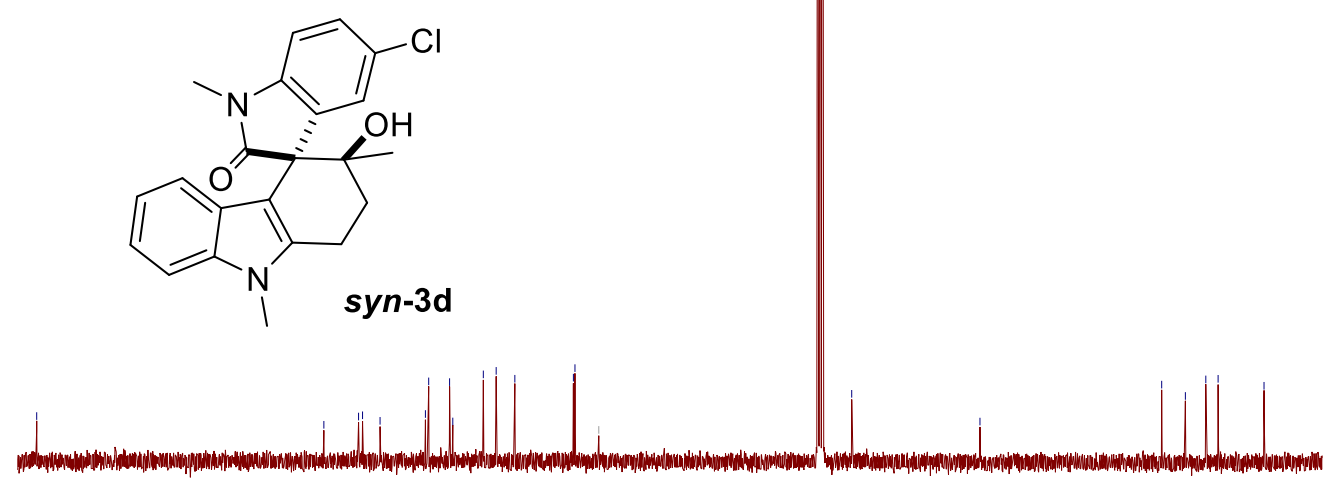

${ }^{13} \mathrm{C}$ NMR Spectrum of Compound 3d $\left(100 \mathrm{MHz}, \mathrm{CDCl}_{3}\right)$. 

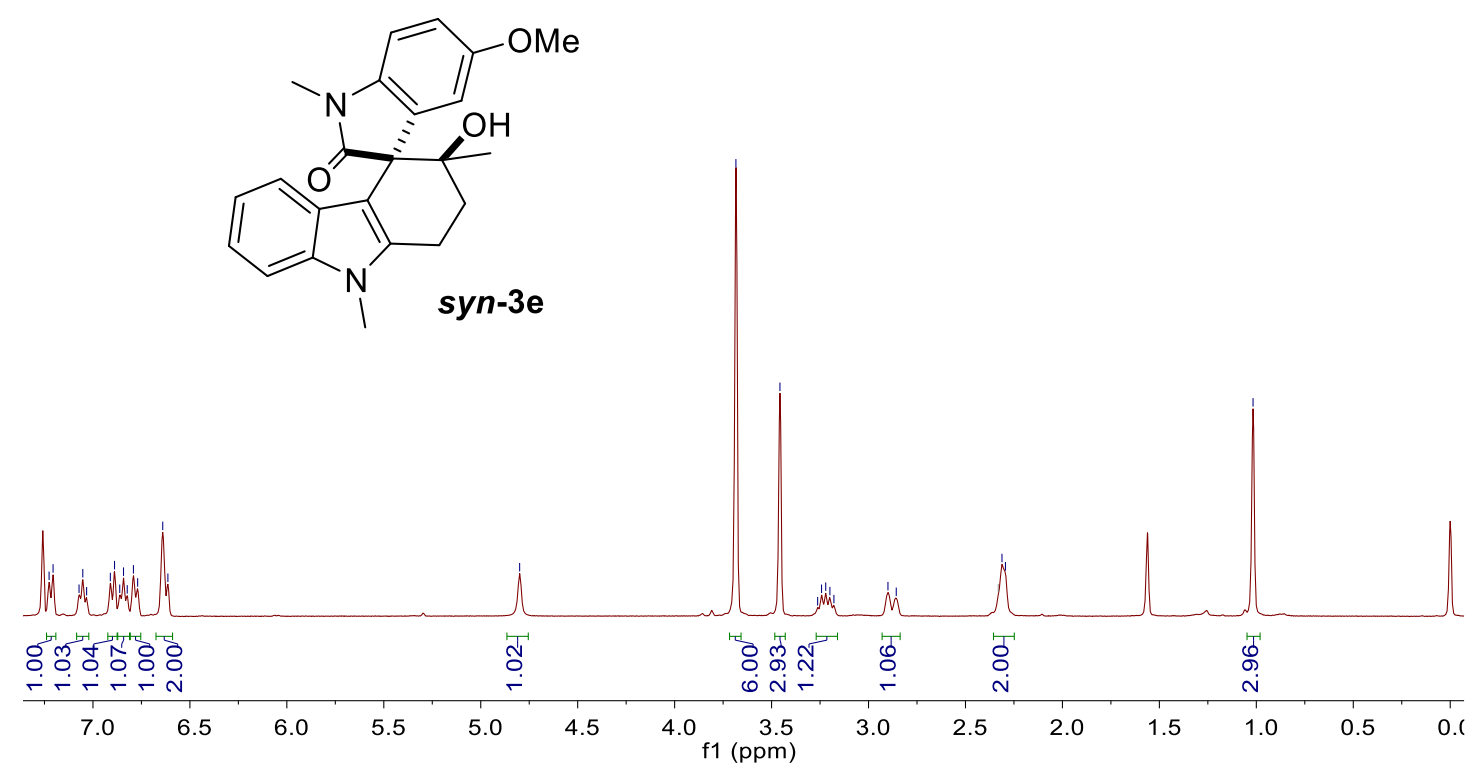

${ }^{1} \mathrm{H}$ NMR Spectrum of Compound $3 \mathbf{e}\left(400 \mathrm{MHz}, \mathrm{CDCl}_{3}\right)$.
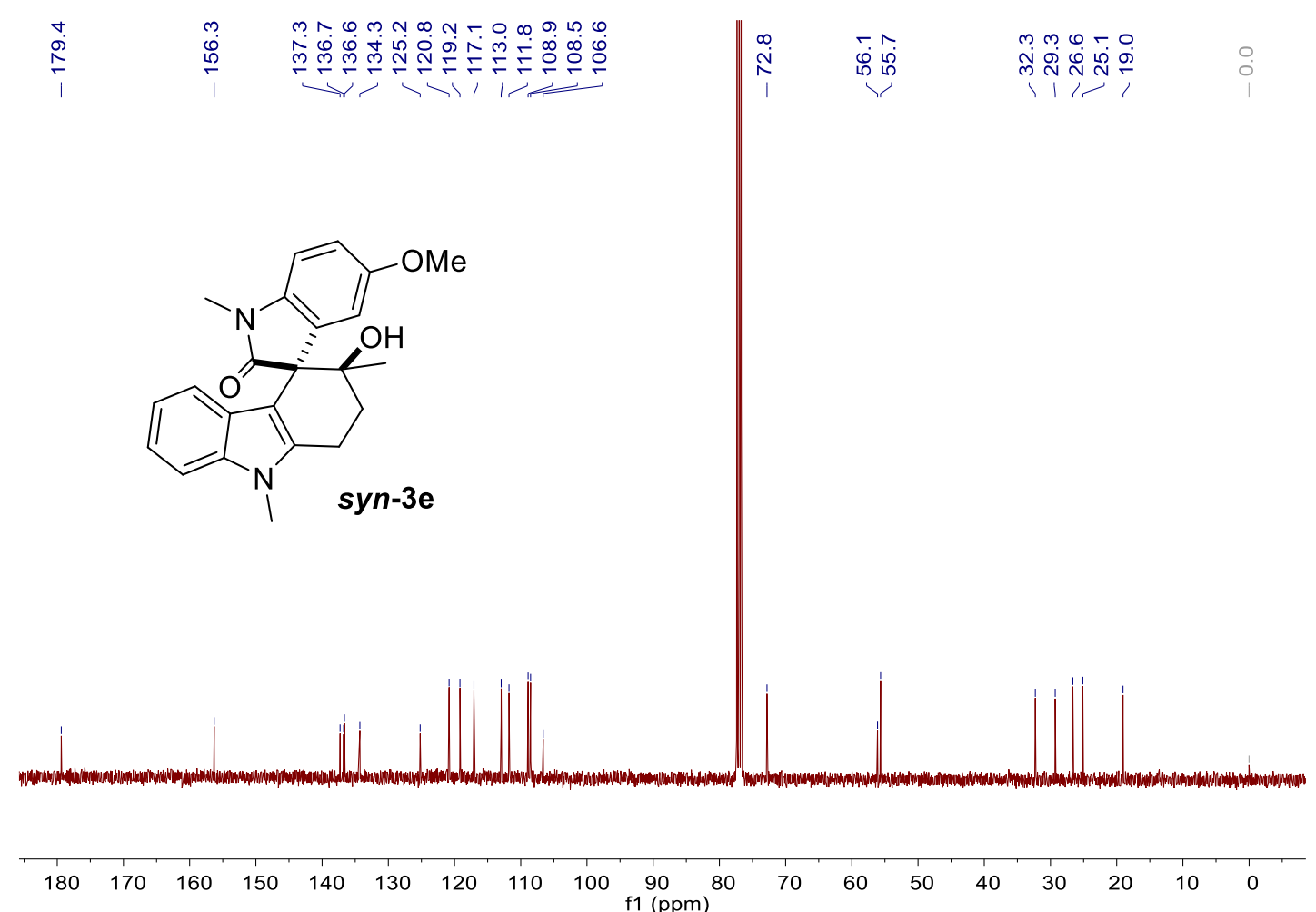

${ }^{13} \mathrm{C}$ NMR Spectrum of Compound $3 \mathbf{e}\left(100 \mathrm{MHz}, \mathrm{CDCl}_{3}\right)$. 

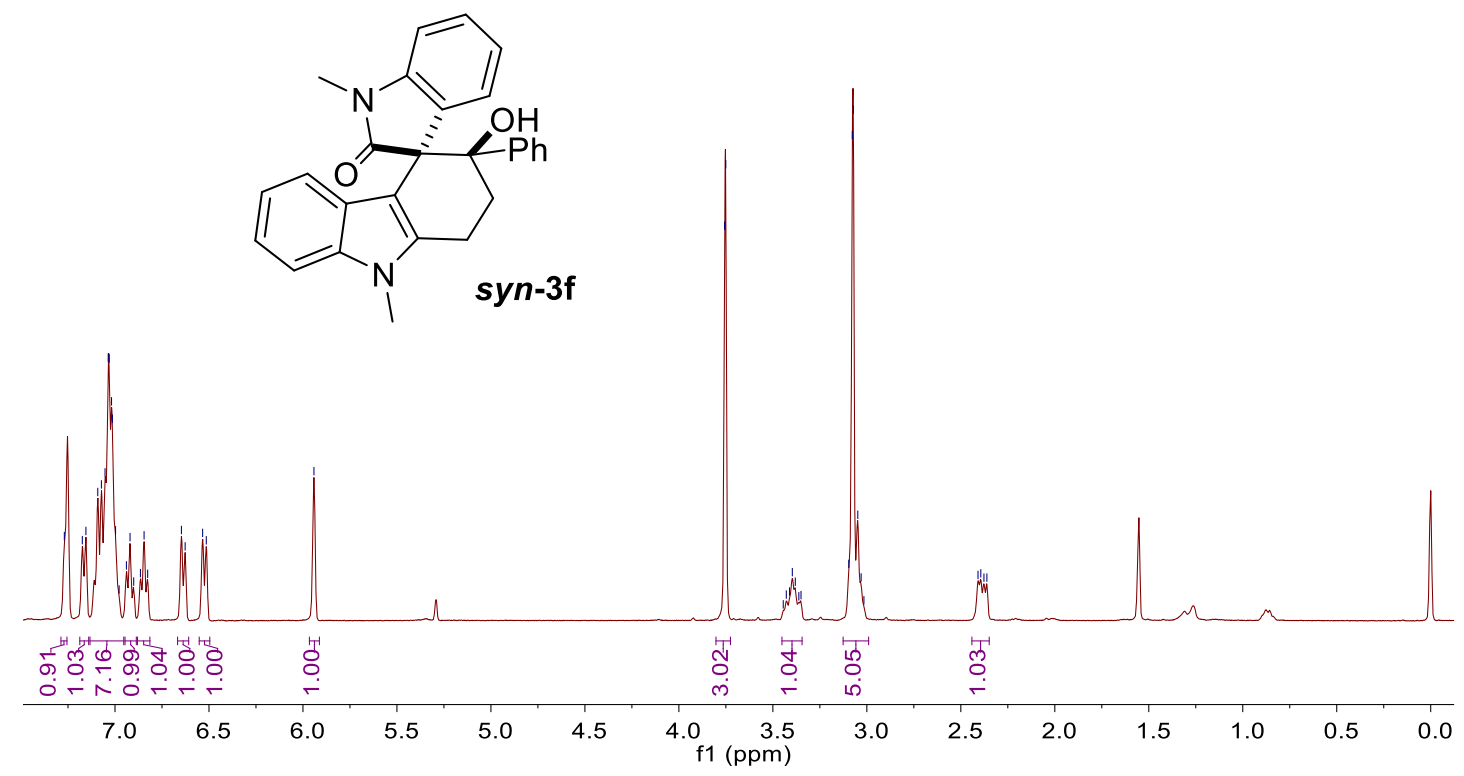

${ }^{1} \mathrm{H}$ NMR Spectrum of Compound $\mathbf{3 f}\left(400 \mathrm{MHz}, \mathrm{CDCl}_{3}\right)$.

華
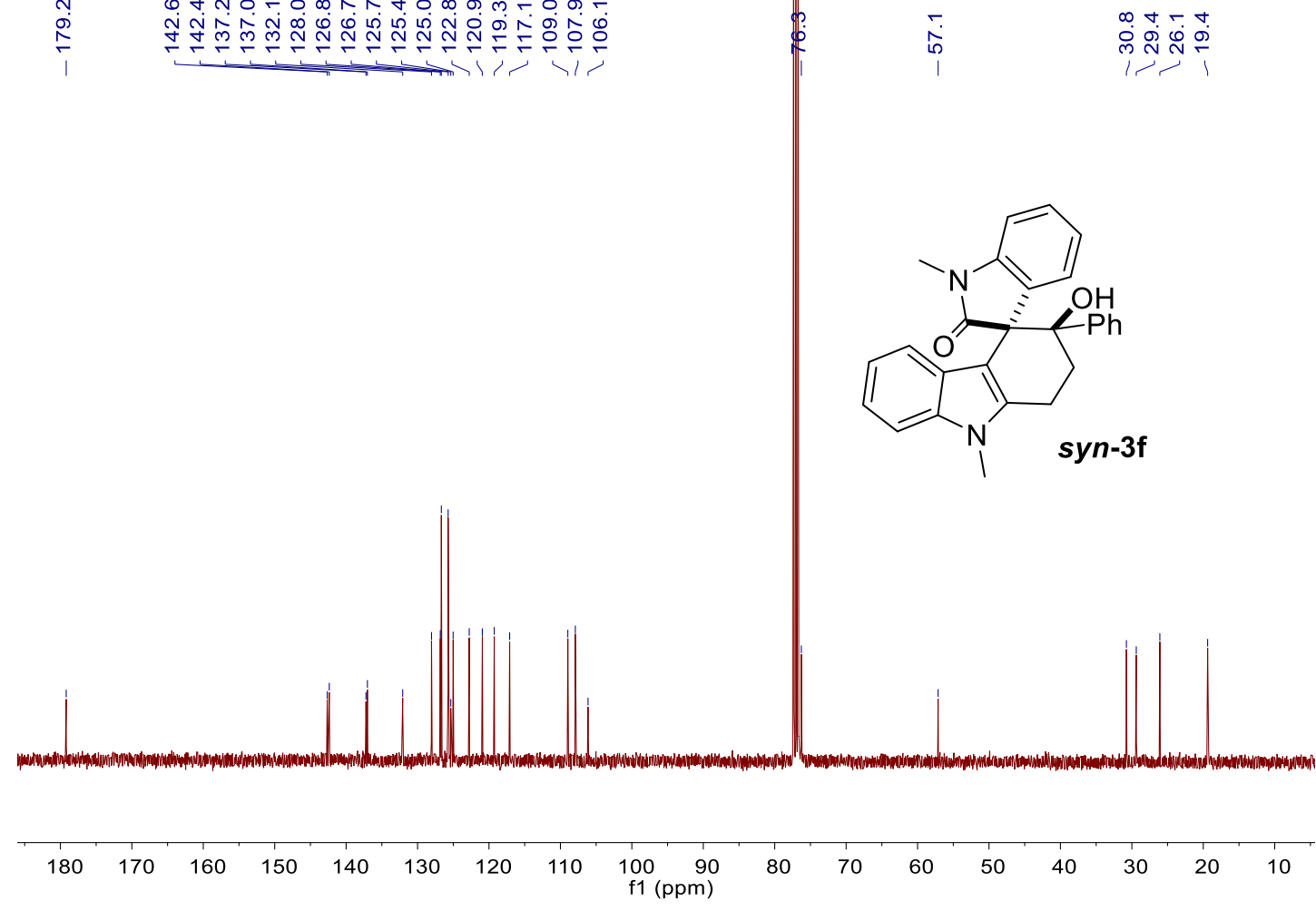

${ }^{13} \mathrm{C}$ NMR Spectrum of Compound $\mathbf{3 f}\left(100 \mathrm{MHz}, \mathrm{CDCl}_{3}\right)$. 


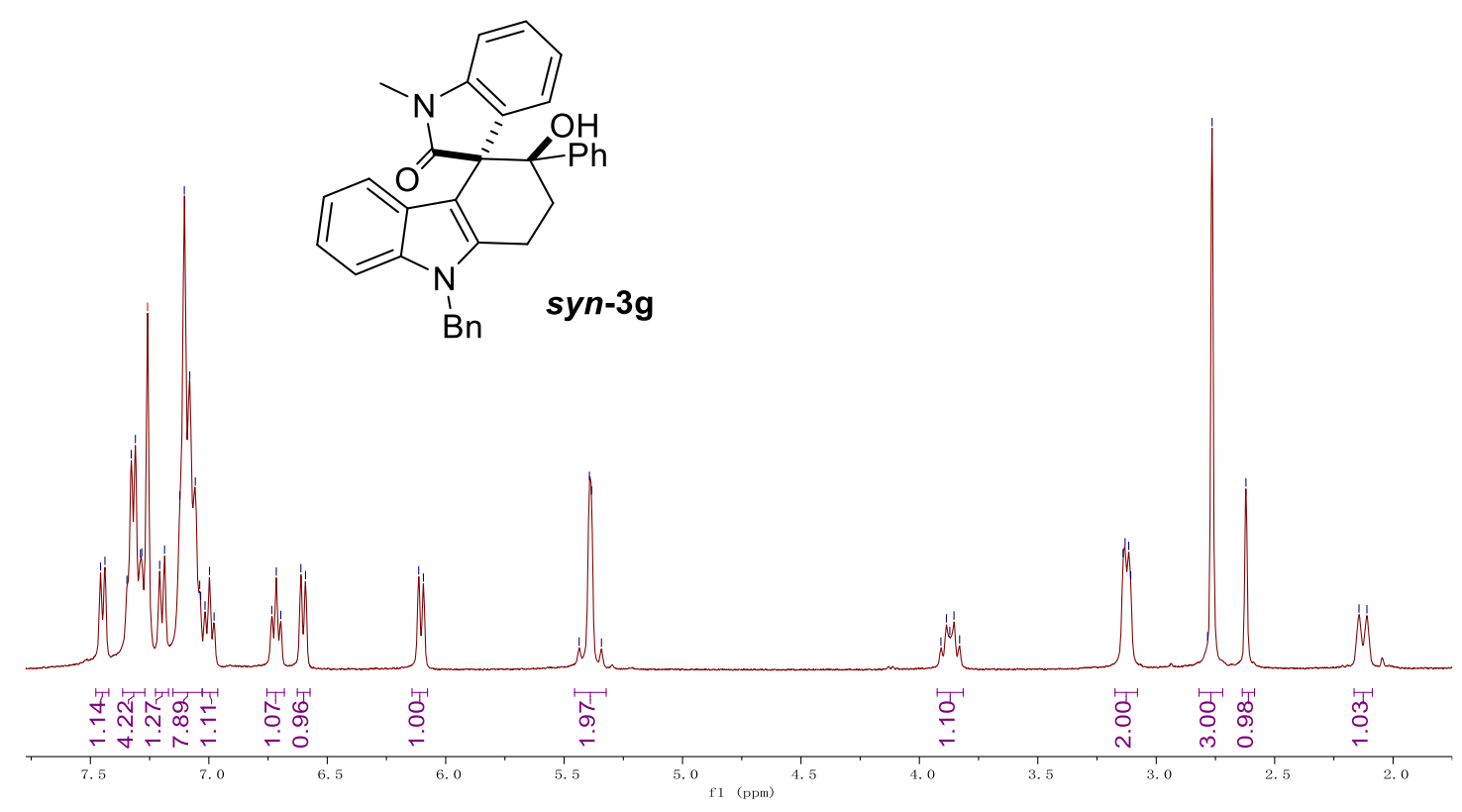

${ }^{1} \mathrm{H}$ NMR Spectrum of Compound $\mathbf{3 g}\left(400 \mathrm{MHz}, \mathrm{CDCl}_{3}\right)$.

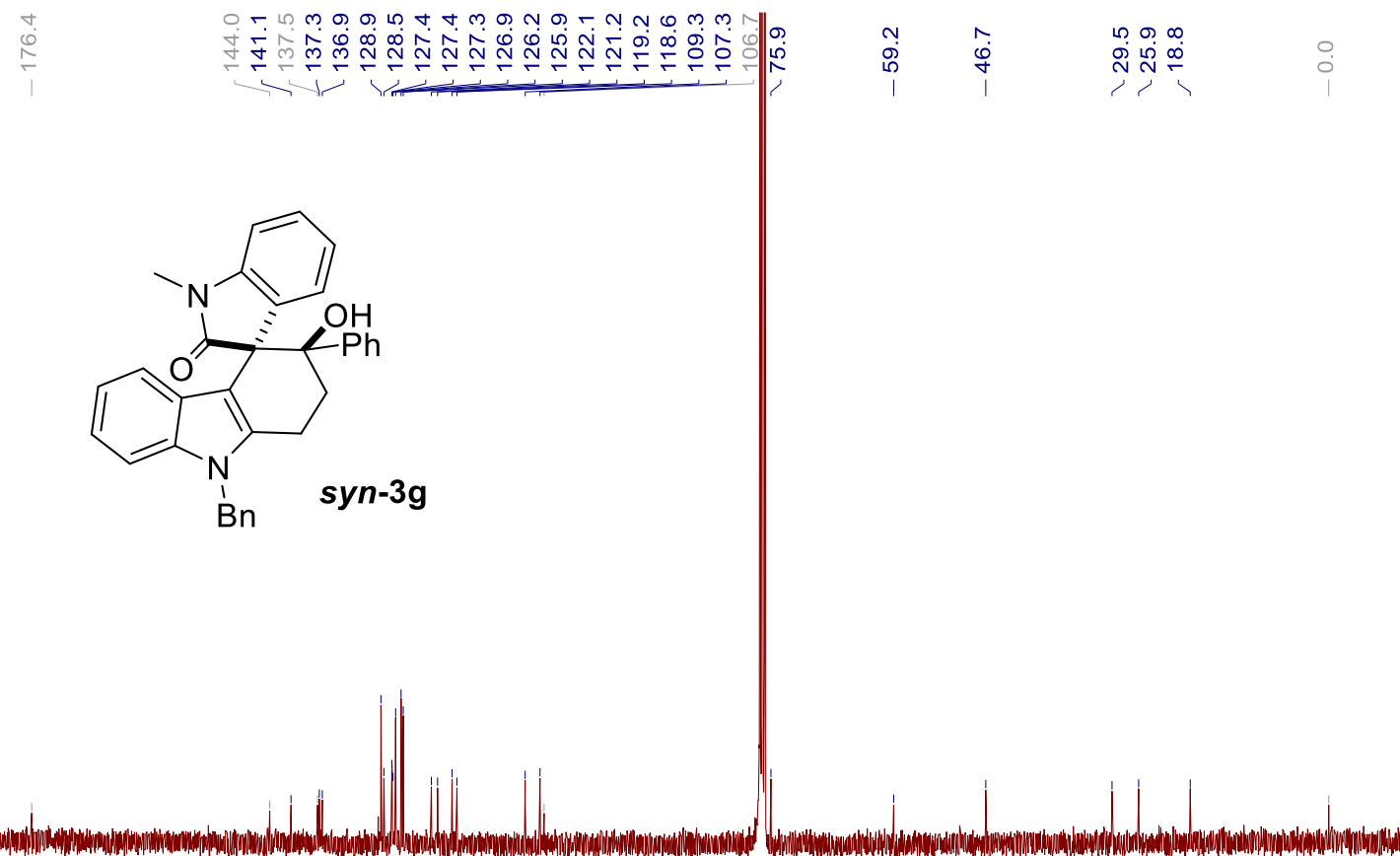

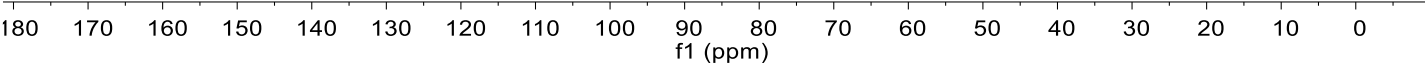

${ }^{13} \mathrm{C}$ NMR Spectrum of Compound $3 g\left(100 \mathrm{MHz}, \mathrm{CDCl}_{3}\right)$ 


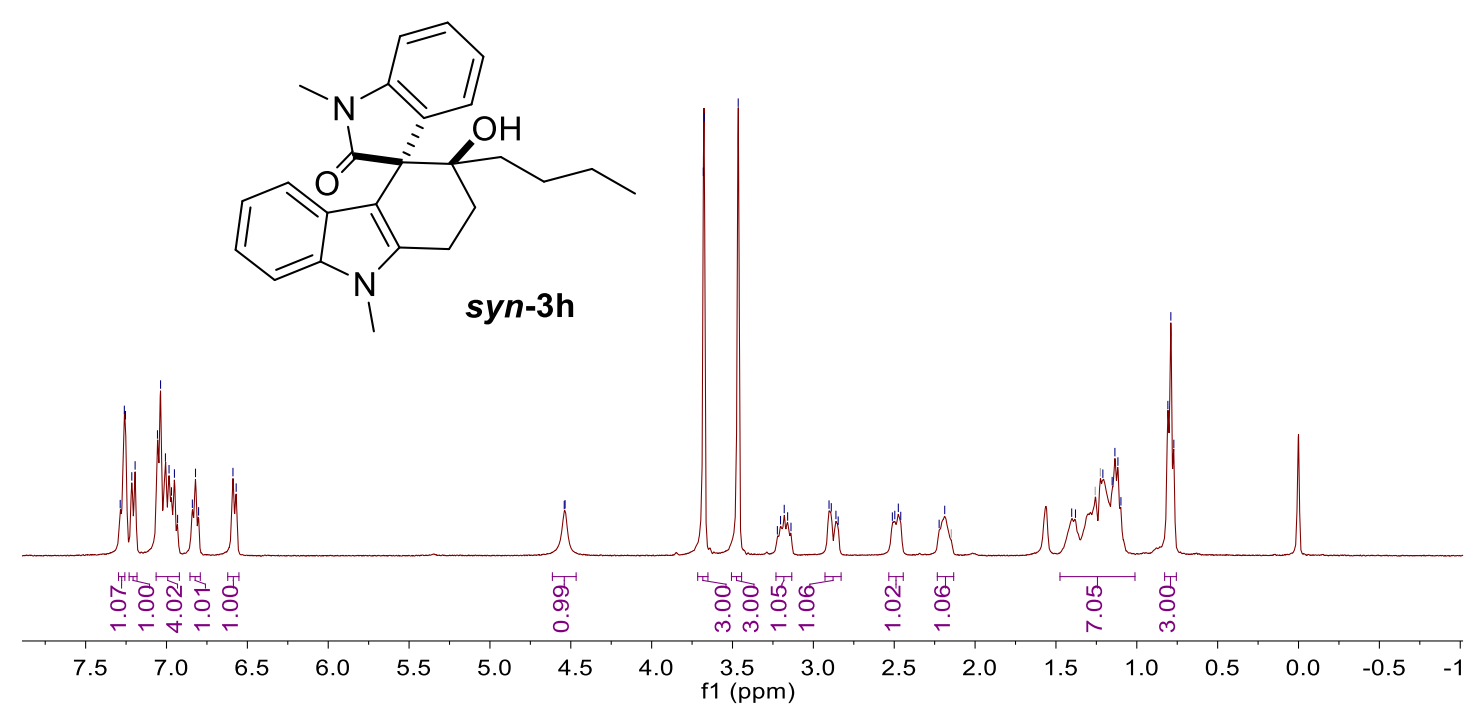

${ }^{1} \mathrm{H}$ NMR Spectrum of Compound $\mathbf{3 h}\left(400 \mathrm{MHz}, \mathrm{CDCl}_{3}\right)$.

$\underset{\substack{\infty \\ i}}{\stackrel{\infty}{i}}$
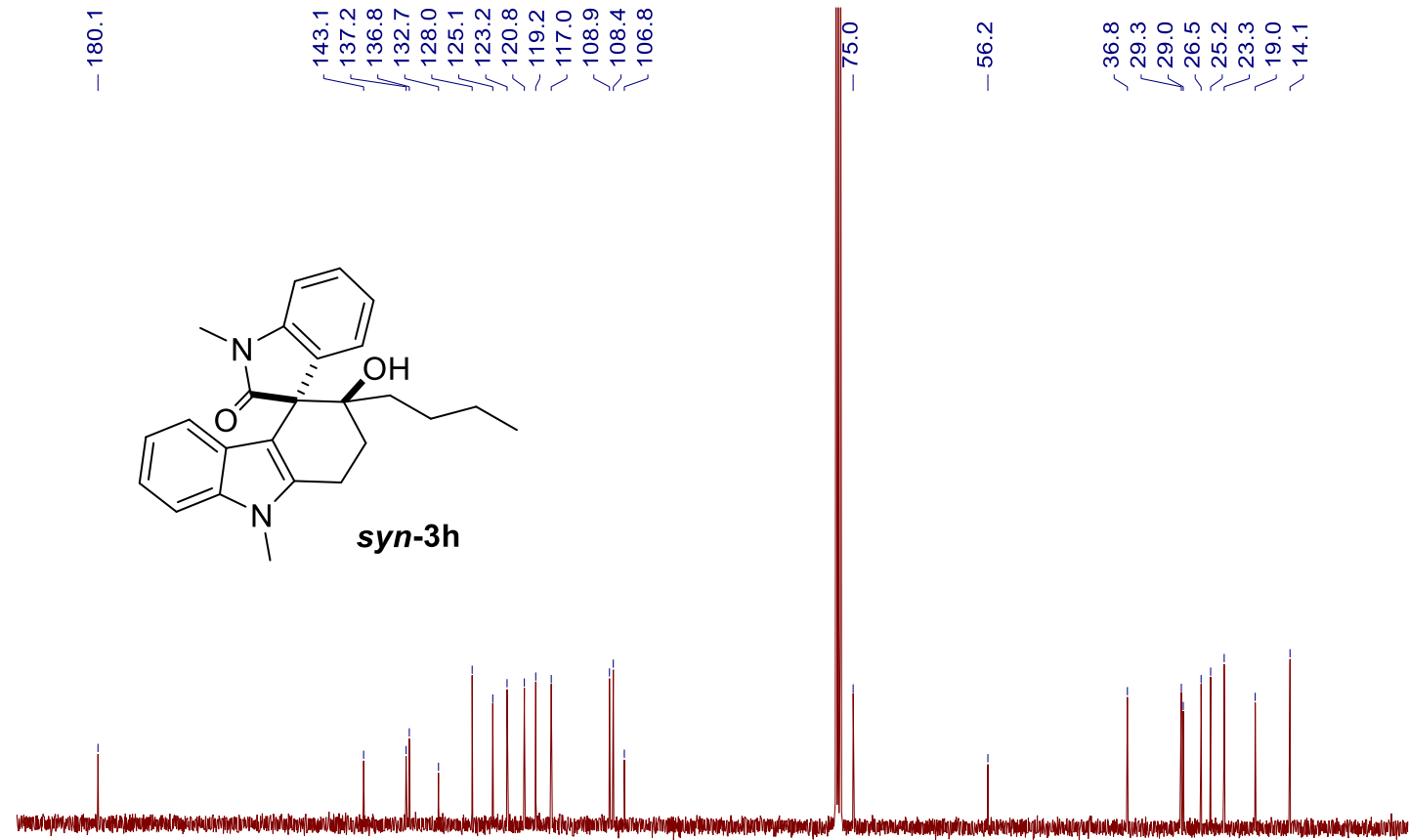

$\begin{array}{rrrrrrrrrrr}190 & 180 & 170 & 160 & 150 & 140 & 130 & 120 & 110 & \begin{array}{l}100 \\ \mathrm{f} 1(\mathrm{ppm})\end{array}\end{array}$

$\begin{array}{lllllllll}80 & 70 & 60 & 50 & 40 & 30 & 20 & 10 & 0\end{array}$

${ }^{13} \mathrm{C}$ NMR Spectrum of Compound $\mathbf{3 h}\left(100 \mathrm{MHz}, \mathrm{CDCl}_{3}\right)$. 

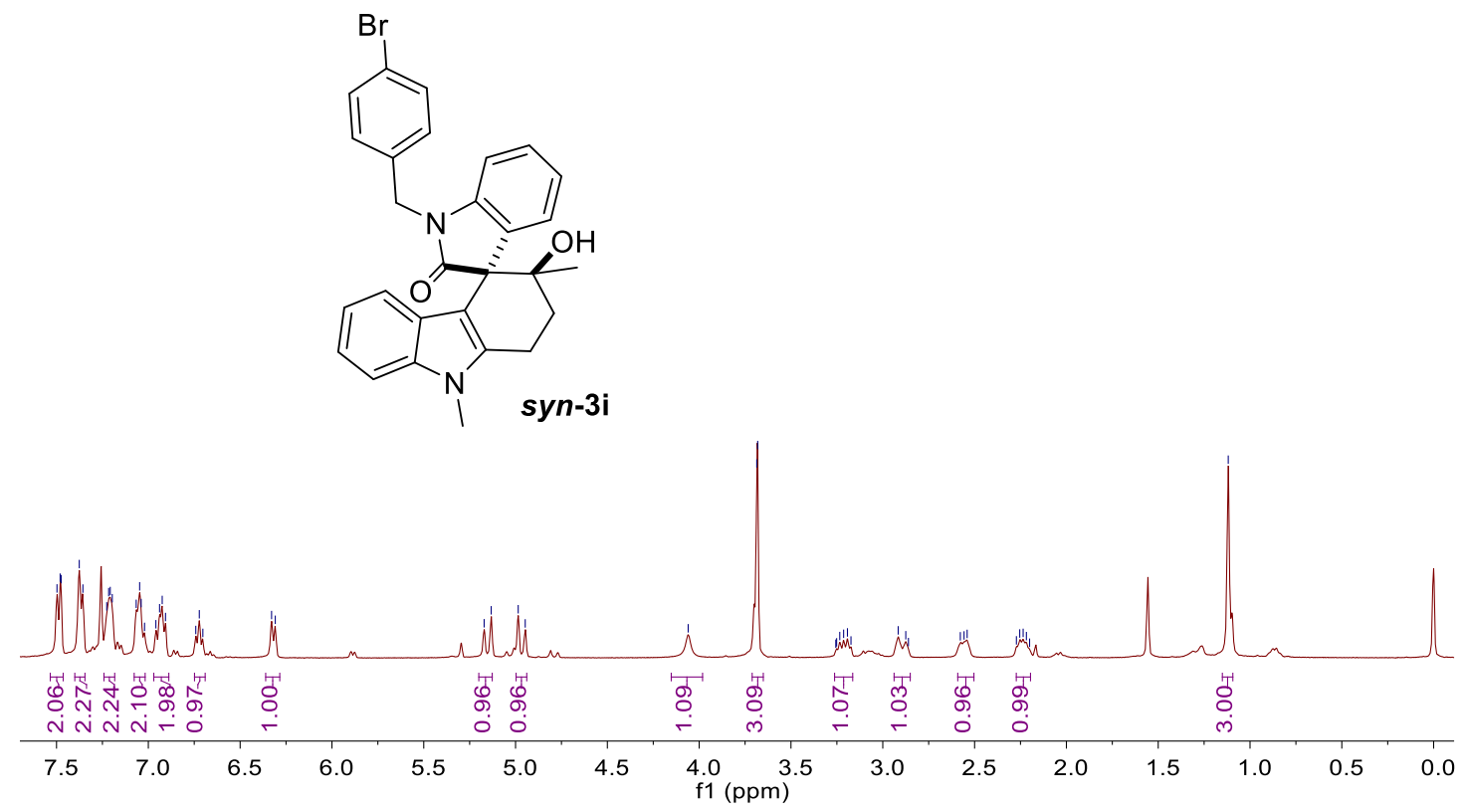

${ }^{1} \mathrm{H}$ NMR Spectrum of Compound 3i (400MHz, $\left.\mathrm{CDCl}_{3}\right)$.

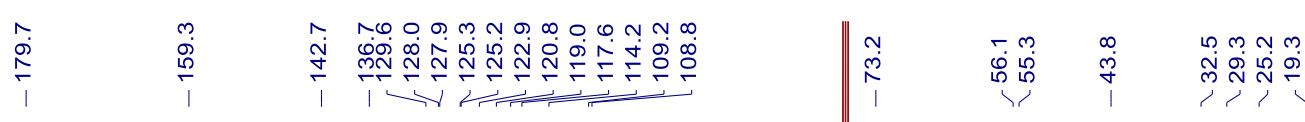<smiles>Cn1c2c(c3ccccc31)[C@@](Cc1ccccc1)(C(=O)N(Cc1ccc(Br)cc1)c1ccccc1)C(O)CC2</smiles>

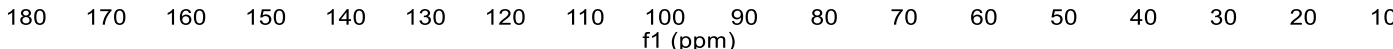

${ }^{13} \mathrm{C}$ NMR Spectrum of Compound $3 \mathbf{i}\left(100 \mathrm{MHz}, \mathrm{CDCl}_{3}\right)$. 


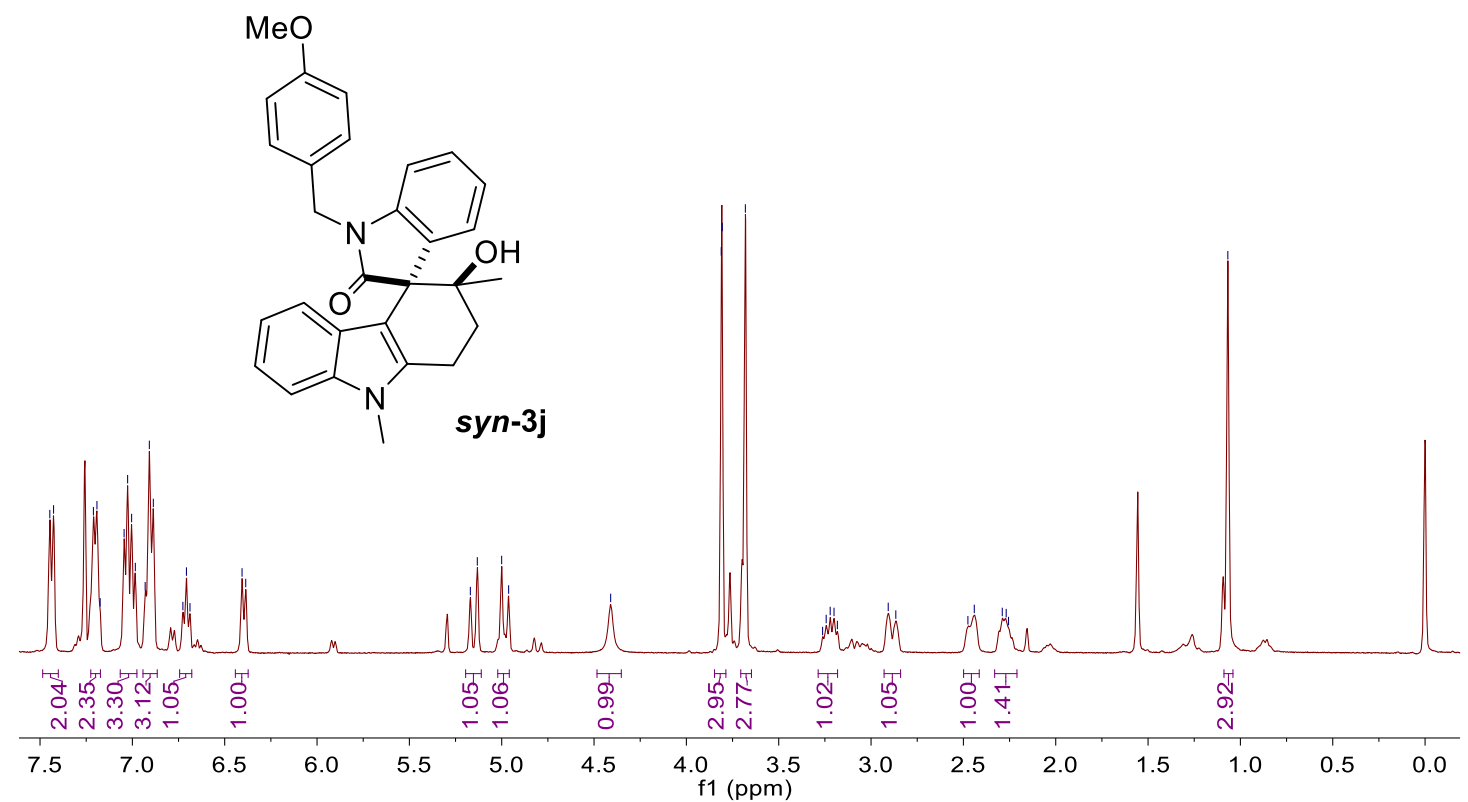

${ }^{1} \mathrm{H}$ NMR Spectrum of Compound $\mathbf{3 j}$ (400MHz, $\mathrm{CDCl}_{3}$ ).
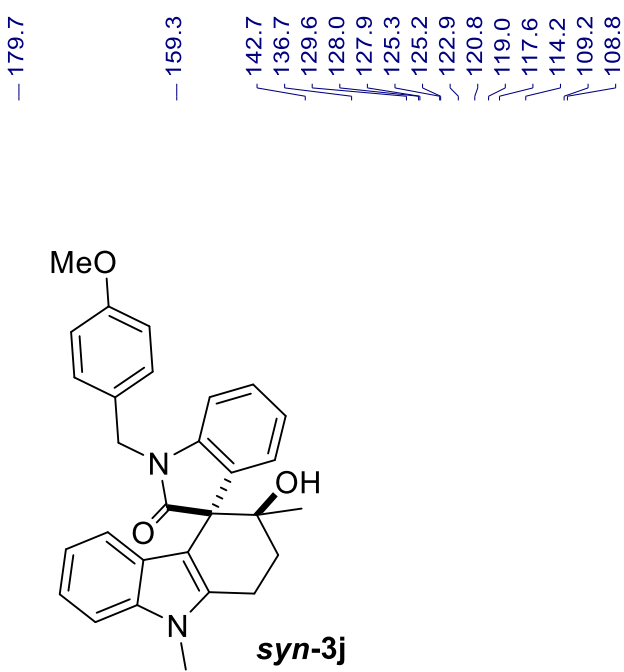

$\begin{array}{llllllllll}180 & 170 & 160 & 150 & 140 & 130 & 120 & 110 & \begin{array}{l}100 \\ \mathrm{f} 1(\mathrm{ppm})\end{array}\end{array}$

${ }^{13} \mathrm{C}$ NMR Spectrum of Compound $\mathbf{3 j}\left(100 \mathrm{MHz}, \mathrm{CDCl}_{3}\right)$. 

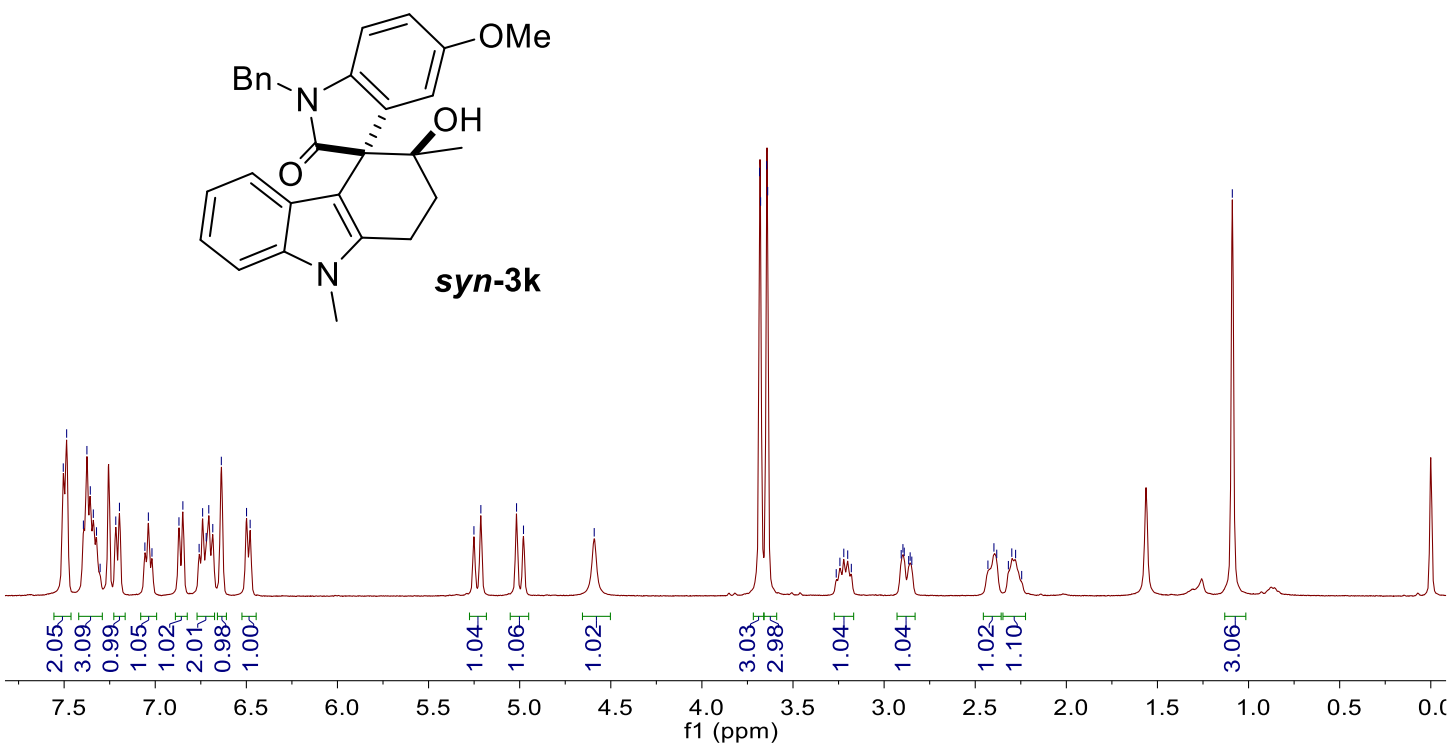

${ }^{1} \mathrm{H}$ NMR Spectrum of Compound 3k (400MHz, $\left.\mathrm{CDCl}_{3}\right)$.

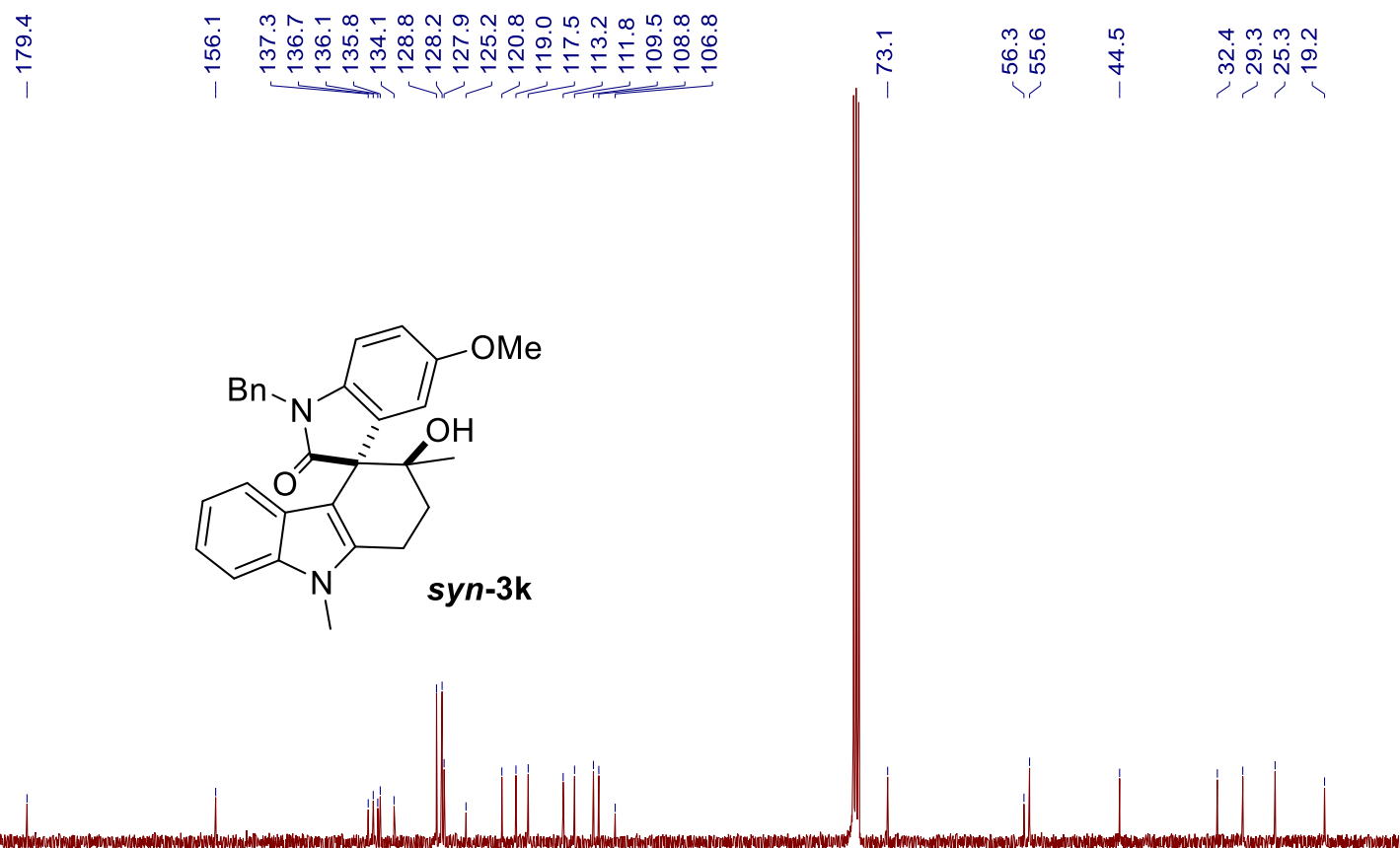

$\begin{array}{lllllllllllllll}180 & 170 & 160 & 150 & 140 & 130 & 120 & 110 & \begin{array}{c}100 \\ \mathrm{f} 1(\mathrm{ppm})\end{array}\end{array}$

${ }^{13} \mathrm{C}$ NMR Spectrum of Compound 3k $\left(100 \mathrm{MHz}, \mathrm{CDCl}_{3}\right)$. 

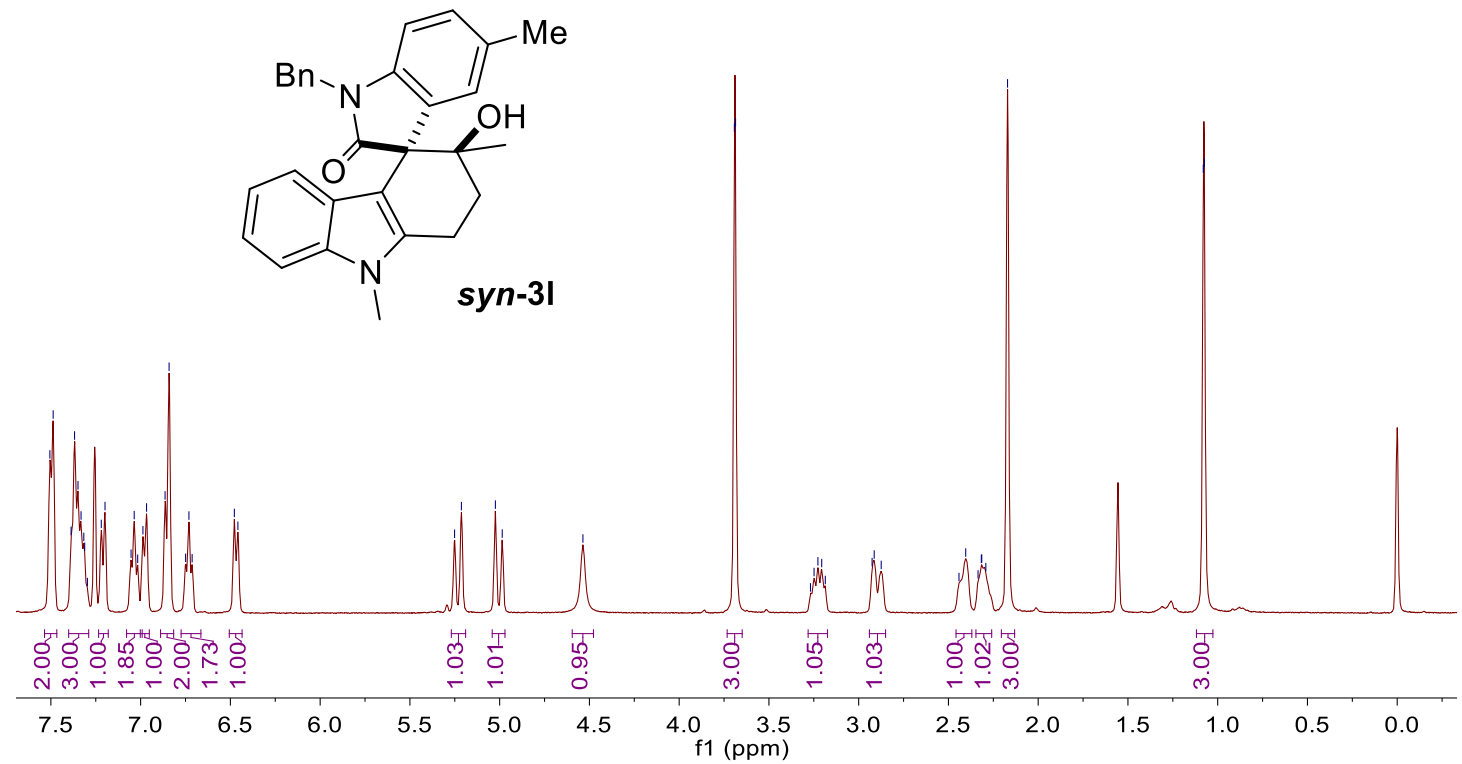

${ }^{1} \mathrm{H}$ NMR Spectrum of Compound 31 (400MHz, $\left.\mathrm{CDCl}_{3}\right)$.
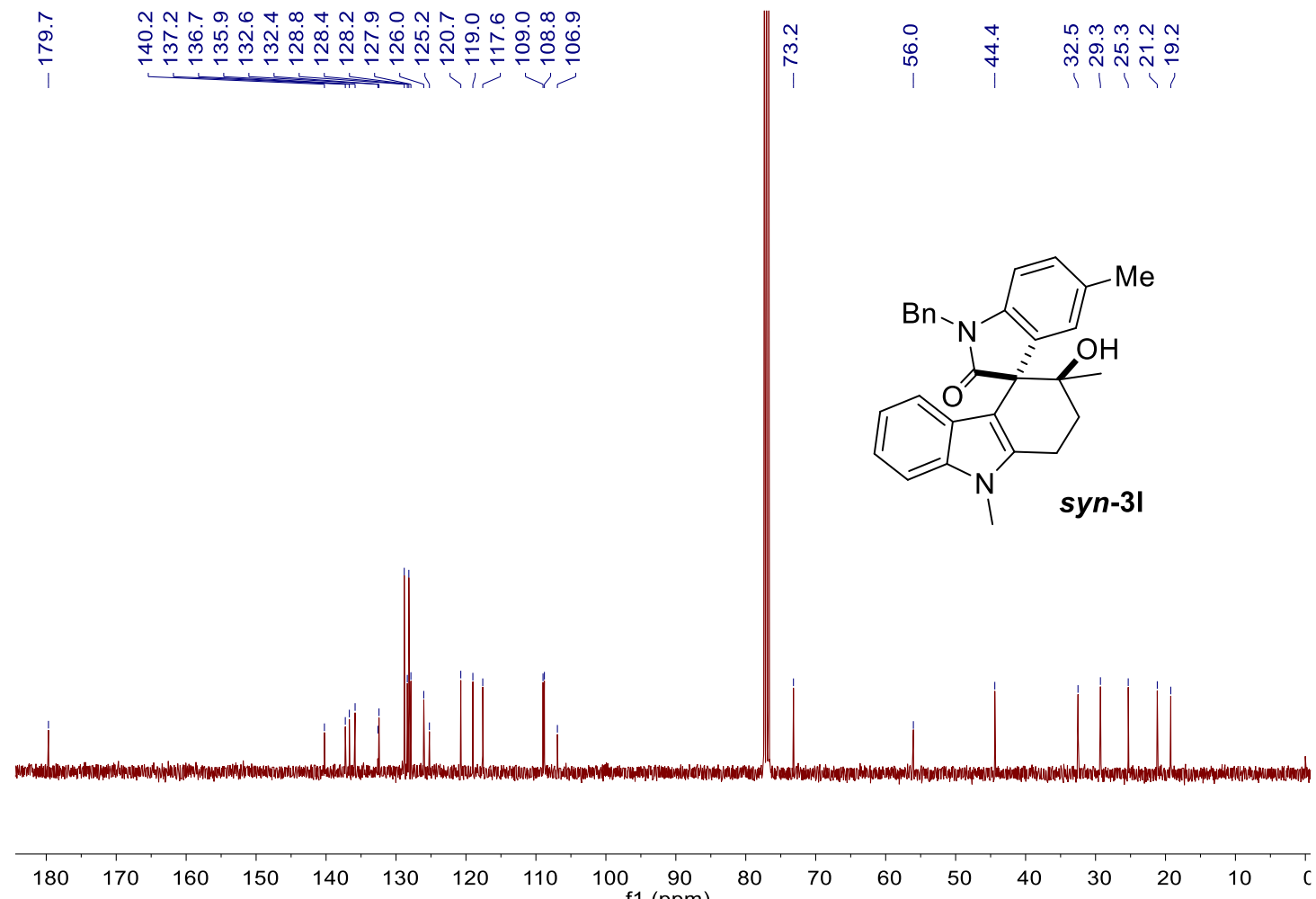

${ }^{13} \mathrm{C}$ NMR Spectrum of Compound 31 (100MHz, $\left.\mathrm{CDCl}_{3}\right)$. 

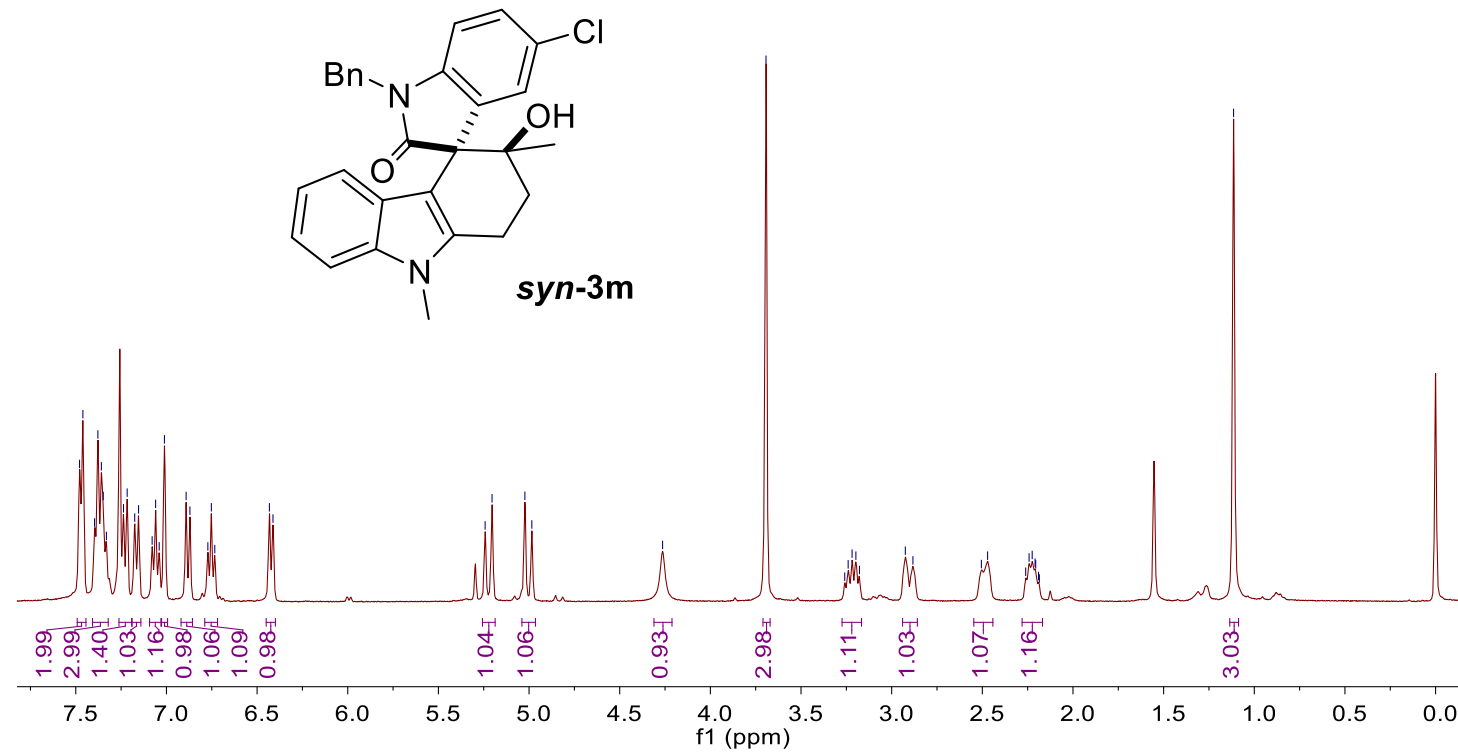

${ }^{1} \mathrm{H}$ NMR Spectrum of Compound $3 \mathbf{m}\left(400 \mathrm{MHz}, \mathrm{CDCl}_{3}\right)$.

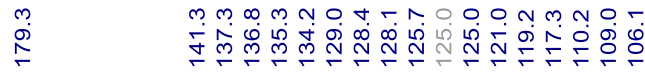

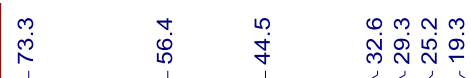<smiles>Cn1c2c(c3ccccc31)[C@@]1(C(=O)N(Cc3ccccc3)c3ccc(Cl)cc31)C(C)(O)CC2</smiles>

${ }^{13} \mathrm{C}$ NMR Spectrum of Compound 3m $\left(100 \mathrm{MHz}, \mathrm{CDCl}_{3}\right)$. 

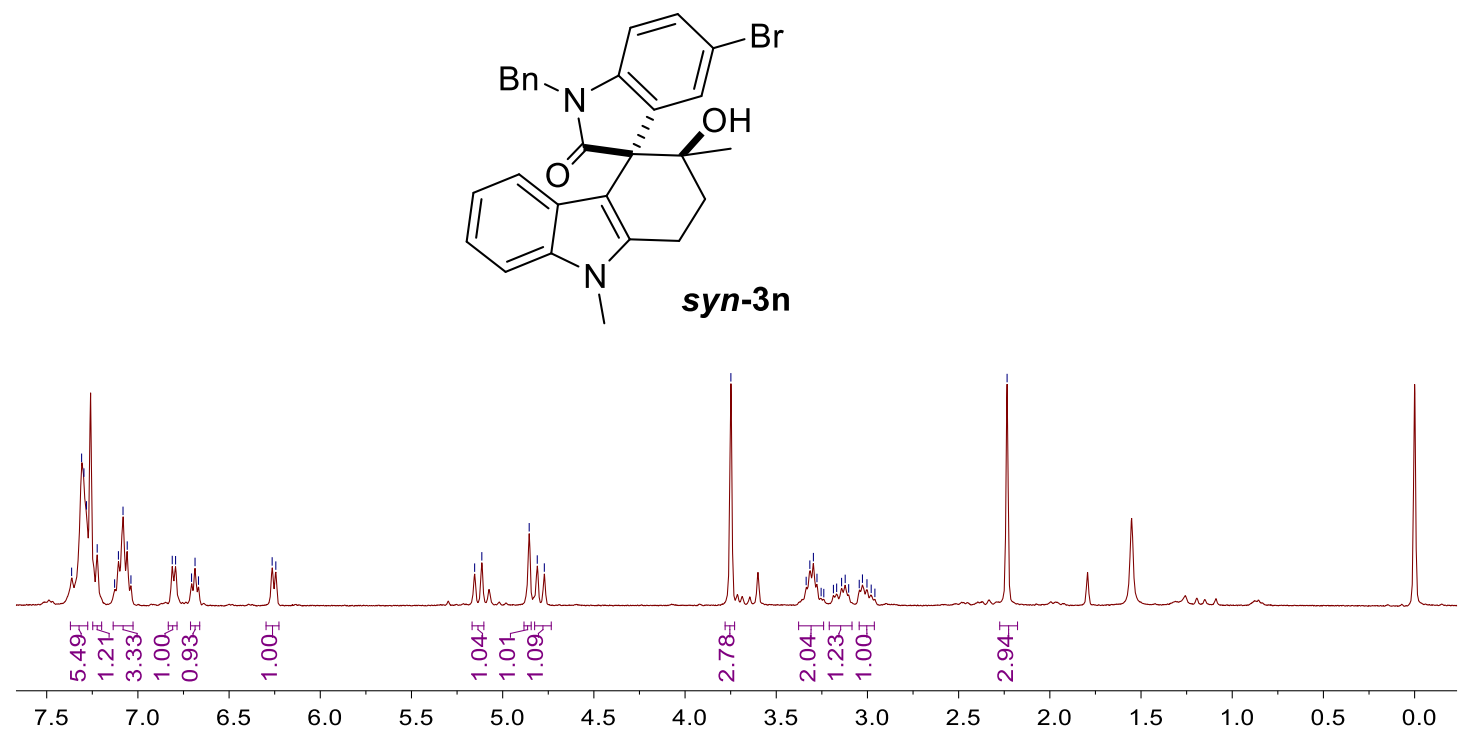

${ }^{1} \mathrm{H}$ NMR Spectrum of Compound 3n $\left(400 \mathrm{MHz}, \mathrm{CDCl}_{3}\right)$.

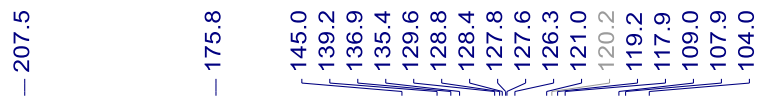

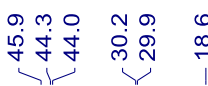<smiles></smiles>

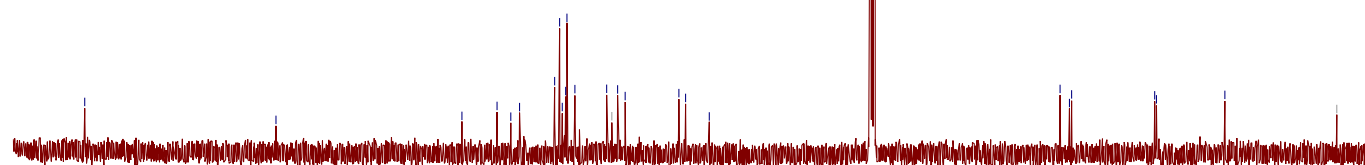

$\begin{array}{lllllllllllllllllllllll}210 & 200 & 190 & 180 & 170 & 160 & 150 & 140 & 130 & 120 & 110 & 100 & 90 & 80 & 70 & 60 & 50 & 40 & 30 & 20 & 10 & 0 & -10\end{array}$

${ }^{13} \mathrm{C}$ NMR Spectrum of Compound 3n $\left(100 \mathrm{MHz}, \mathrm{CDCl}_{3}\right)$. 


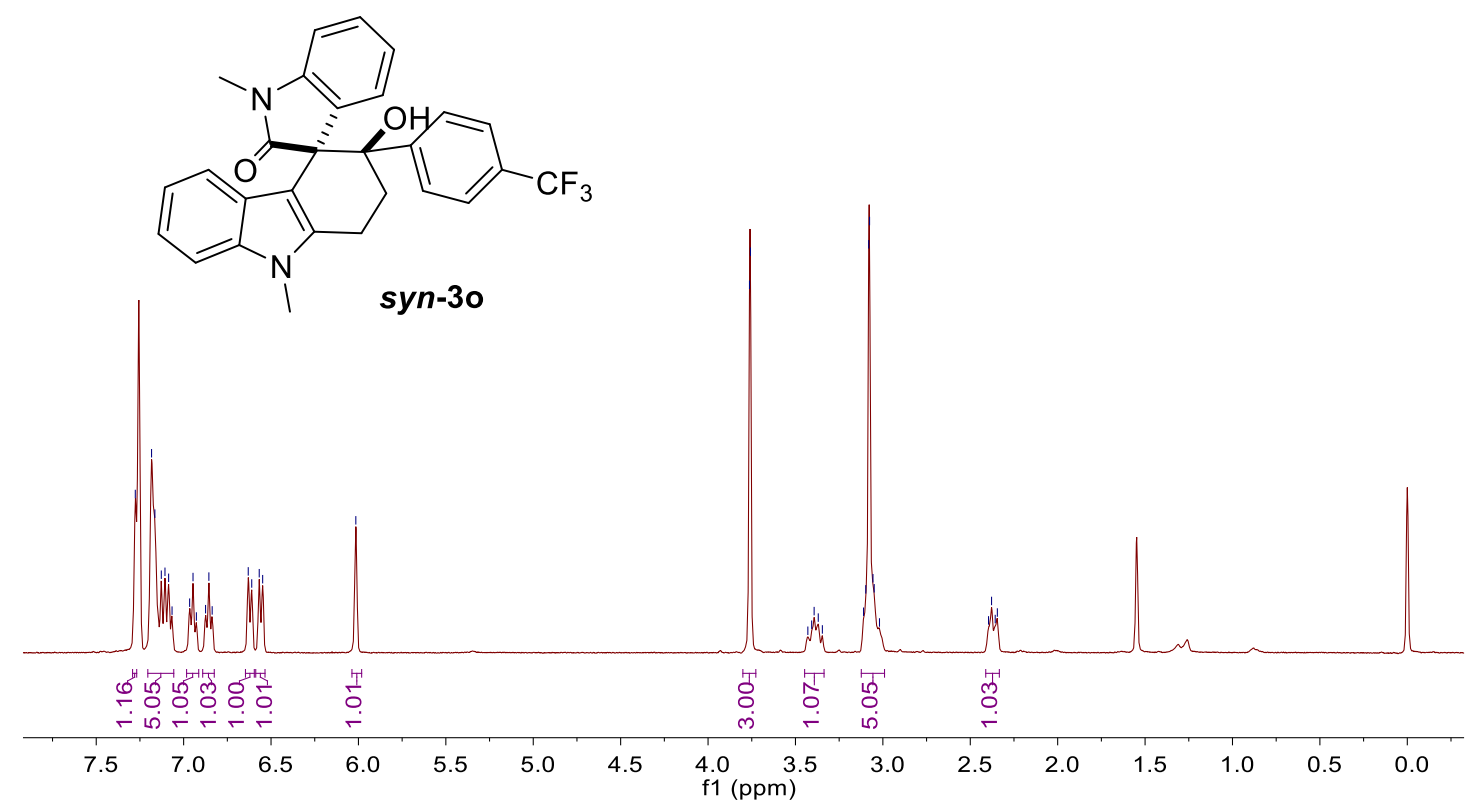

${ }^{1} \mathrm{H}$ NMR Spectrum of Compound $30\left(400 \mathrm{MHz}, \mathrm{CDCl}_{3}\right)$.

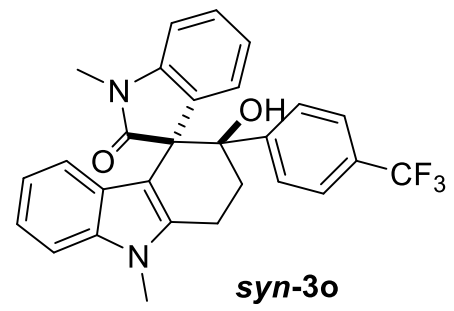

$\begin{array}{lllllllllllllllllllllll}210 & 200 & 190 & 180 & 170 & 160 & 150 & 140 & 130 & 120 & \begin{array}{c}110 \\ \mathrm{f} 1\end{array}(\mathrm{ppm}) & 90 & 80 & 70 & 60 & 50 & 40 & 30 & 20 & 10 & 0 & -10\end{array}$

${ }^{13} \mathrm{C}$ NMR Spectrum of Compound $30\left(100 \mathrm{MHz}, \mathrm{CDCl}_{3}\right)$. 

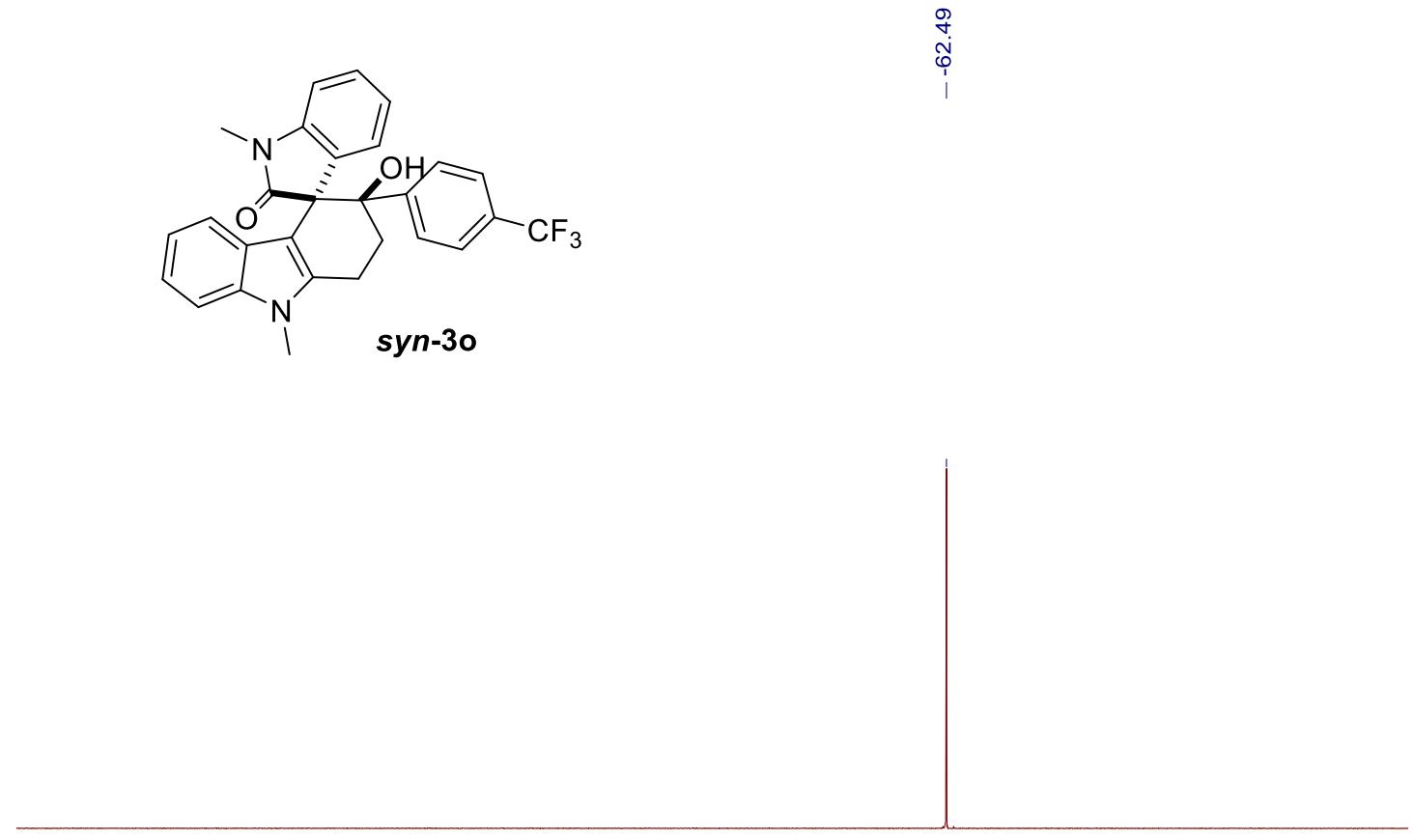

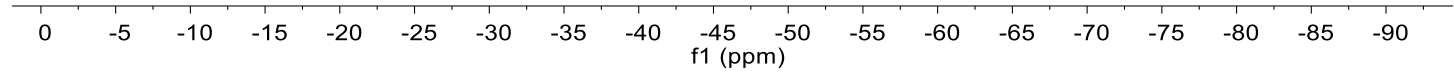

${ }^{19} \mathrm{~F}$ NMR Spectrum of Compound $30\left(376 \mathrm{MHz}, \mathrm{CDCl}_{3}\right)$.

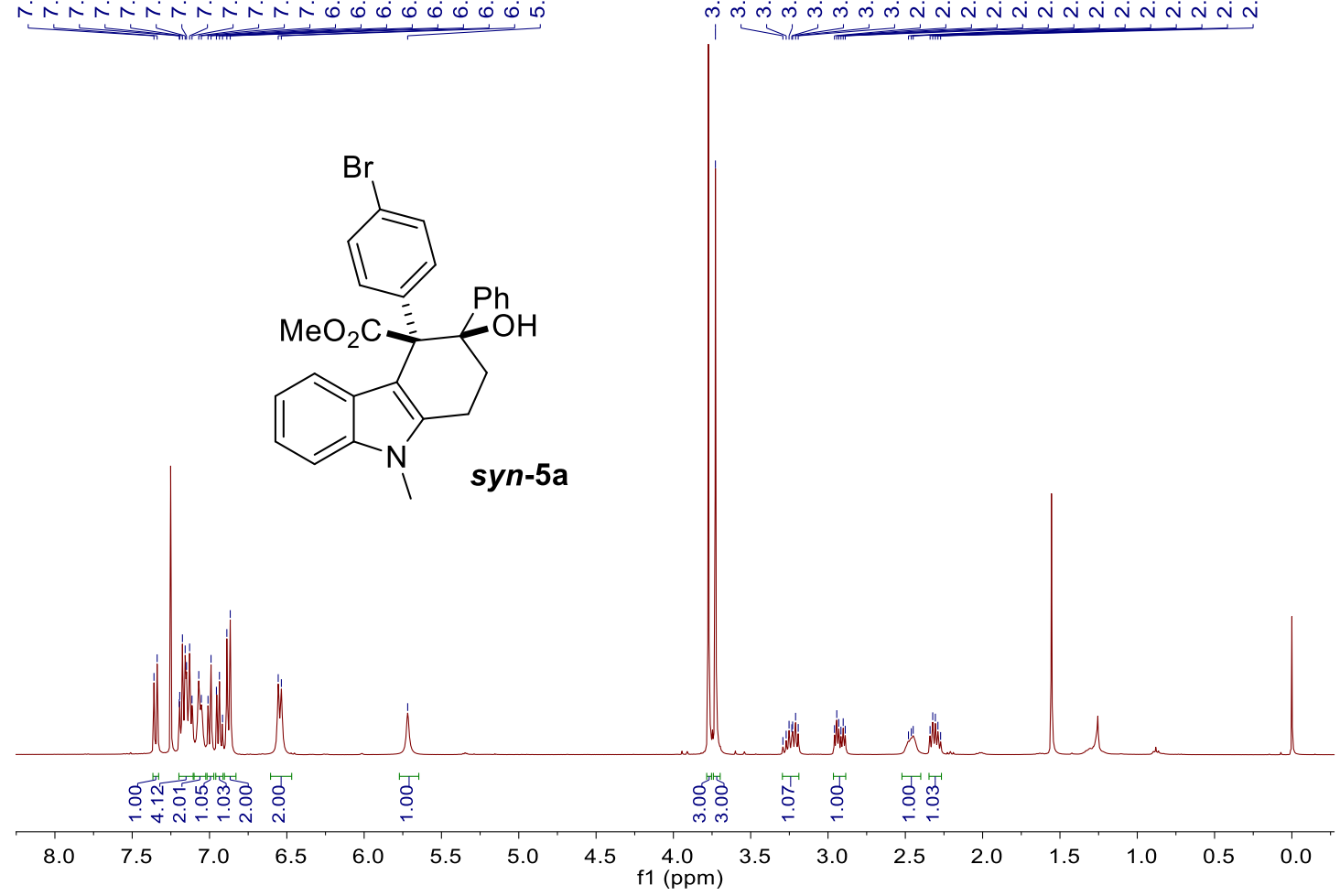

${ }^{1} \mathrm{H}$ NMR Spectrum of Compound 5a $\left(400 \mathrm{MHz}, \mathrm{CDCl}_{3}\right)$. 

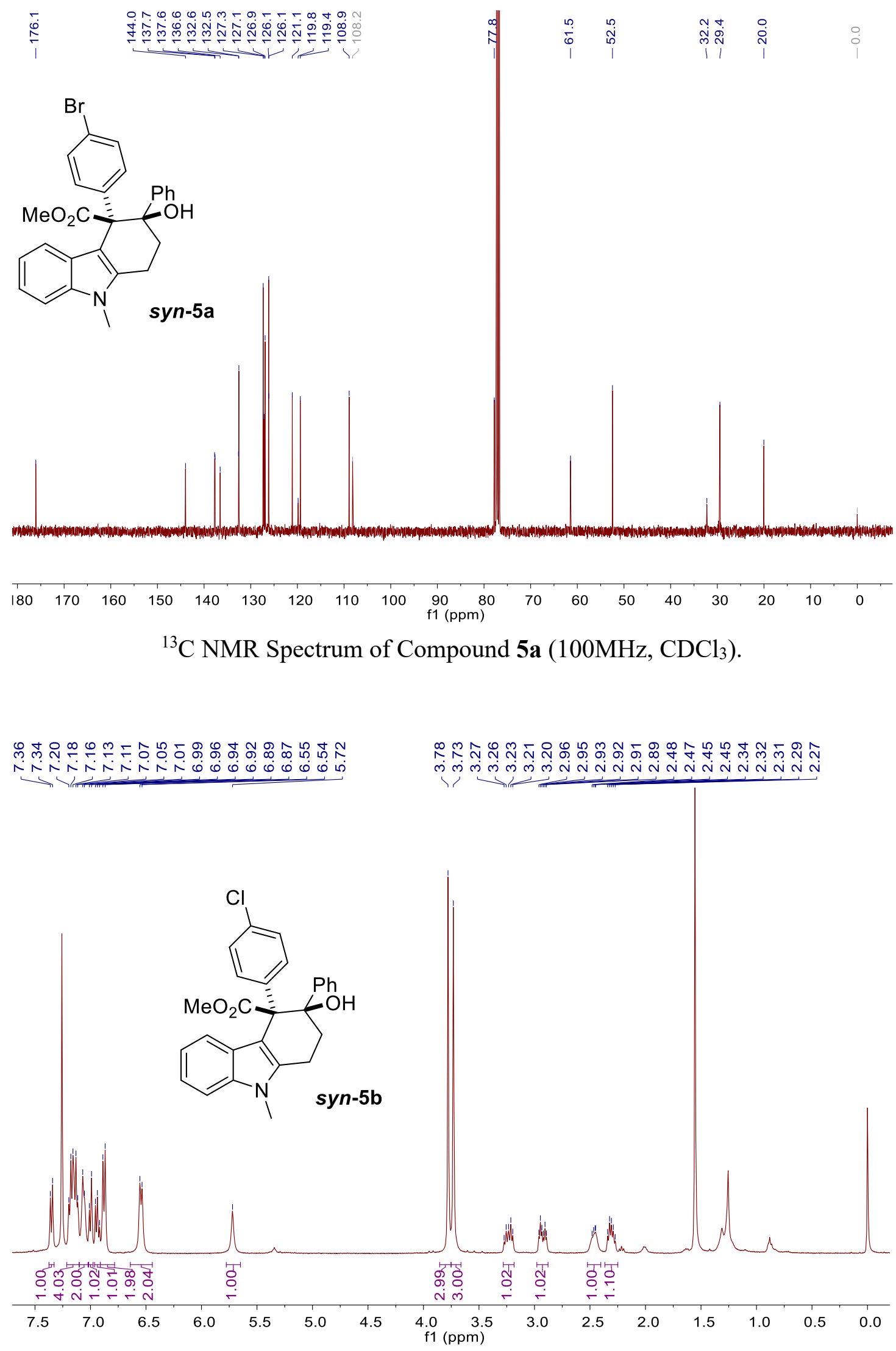

${ }^{1} \mathrm{H}$ NMR Spectrum of Compound $\mathbf{5 b}\left(400 \mathrm{MHz}, \mathrm{CDCl}_{3}\right)$. 
$\tau$ -

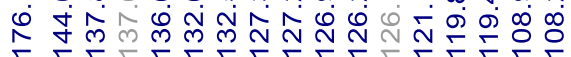
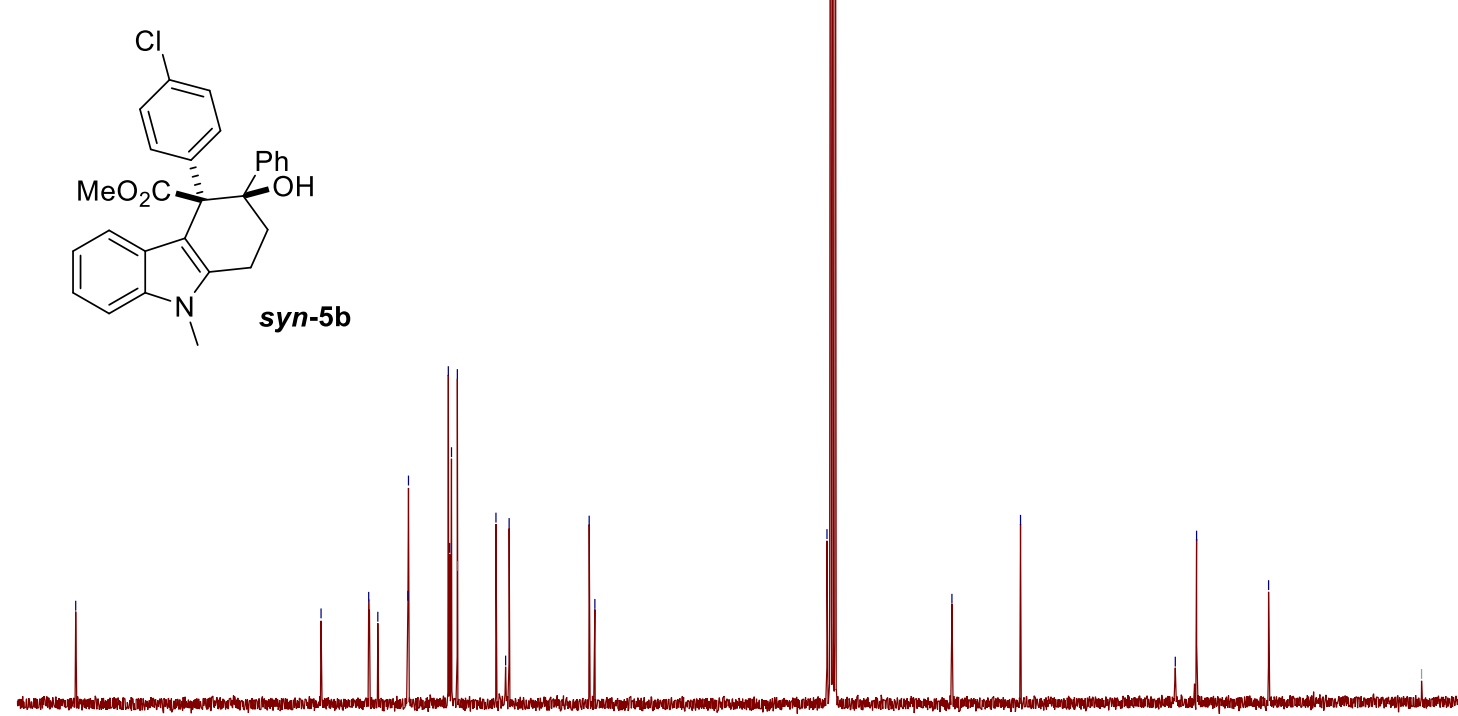

$\begin{array}{llllllllllllllllll}180 & 170 & 160 & 150 & 140 & 130 & 120 & 110 & 100 & \underset{\mathrm{f} 1}{(\mathrm{ppm})} \mathbf{8 0} & 70 & 60 & 50 & 40 & 30 & 20 & 10 & 0\end{array}$

${ }^{13} \mathrm{C}$ NMR Spectrum of Compound $\mathbf{5 b}\left(100 \mathrm{MHz}, \mathrm{CDCl}_{3}\right)$.
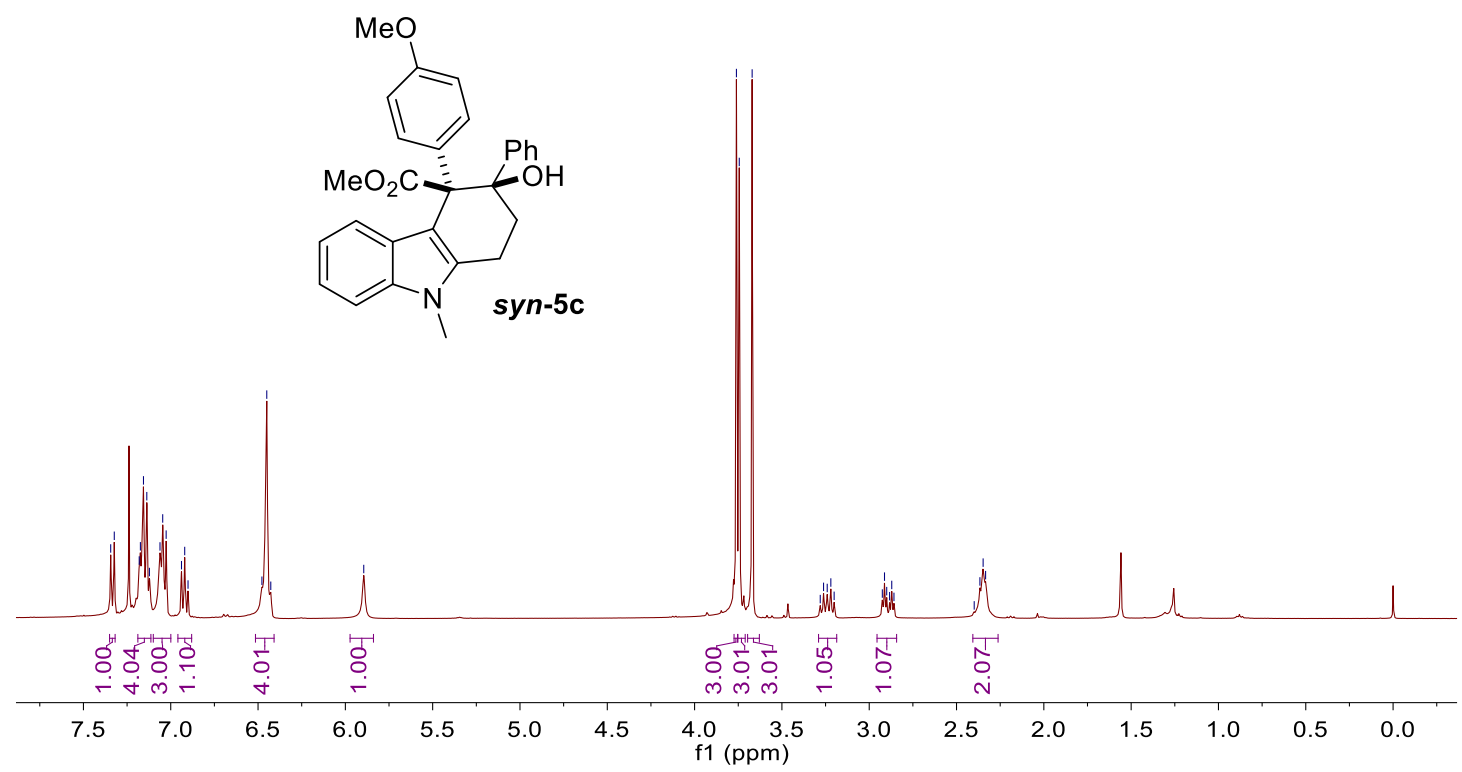

${ }^{1} \mathrm{H}$ NMR Spectrum of Compound $\mathbf{5 c}\left(400 \mathrm{MHz}, \mathrm{CDCl}_{3}\right)$. 

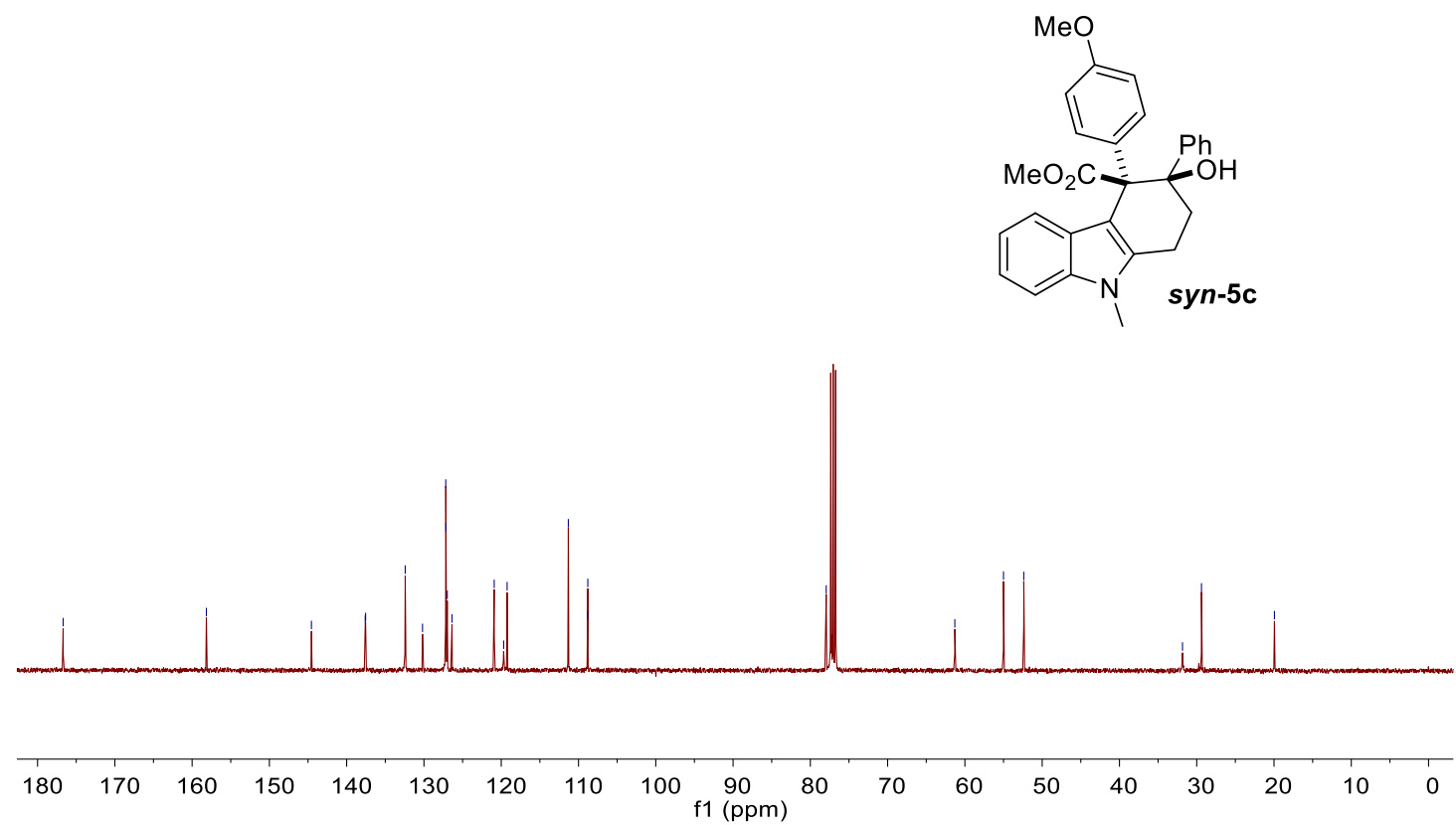

${ }^{13} \mathrm{C}$ NMR Spectrum of Compound $\mathbf{5 c}\left(100 \mathrm{MHz}, \mathrm{CDCl}_{3}\right)$.

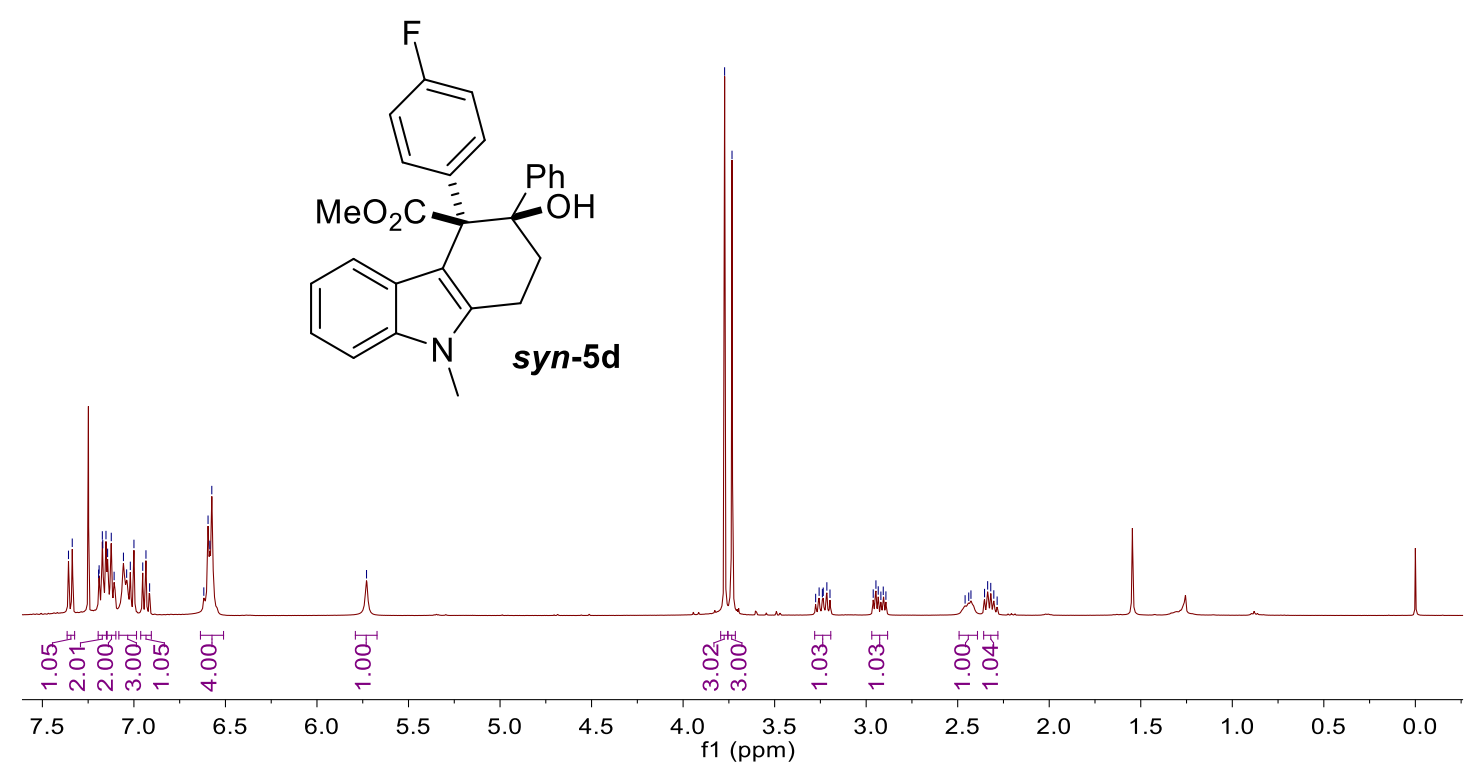

${ }^{1} \mathrm{H}$ NMR Spectrum of Compound $\mathbf{5 d}\left(400 \mathrm{MHz}, \mathrm{CDCl}_{3}\right)$. 

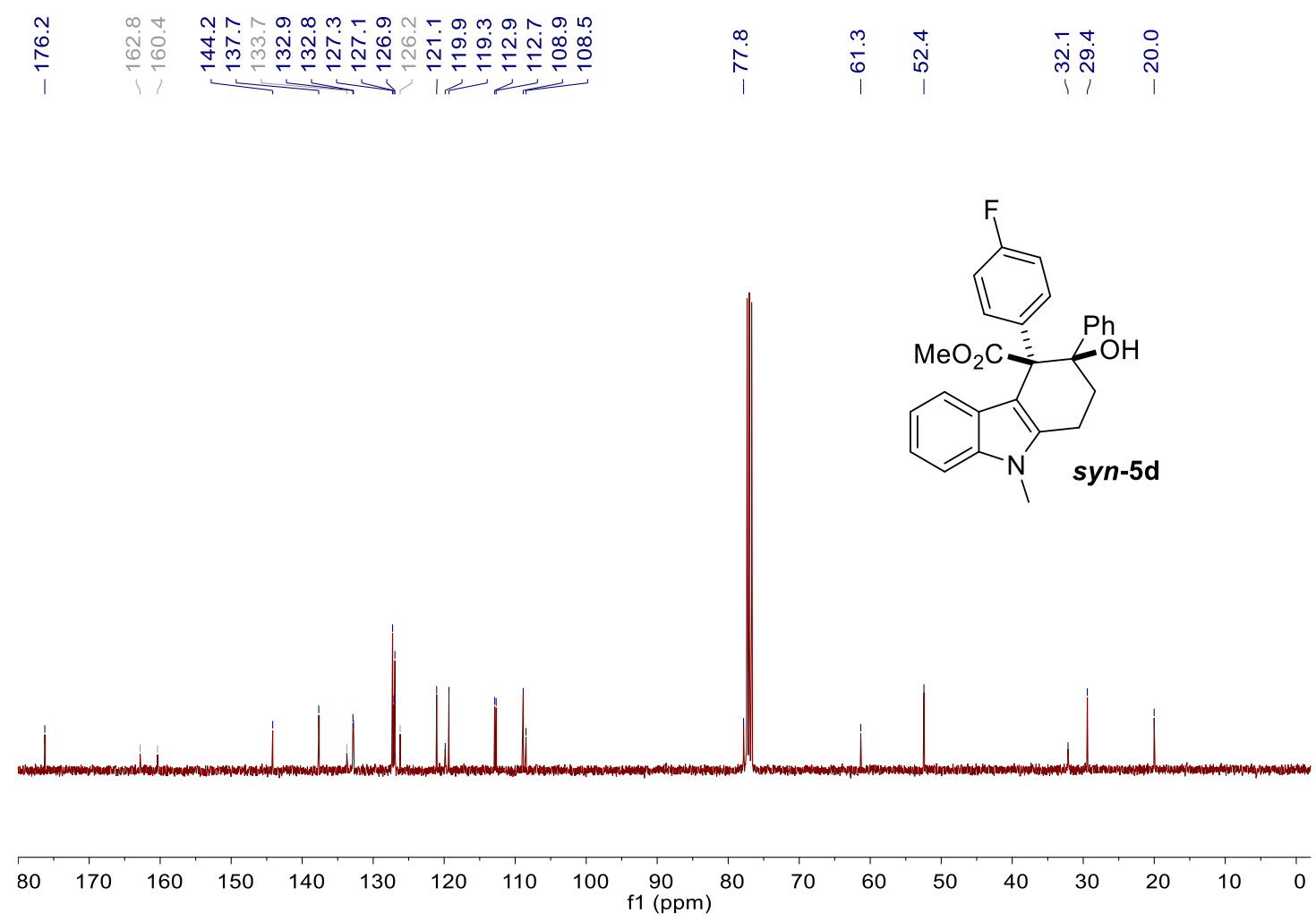

${ }^{13} \mathrm{C}$ NMR Spectrum of Compound $\mathbf{5 d}\left(100 \mathrm{MHz}, \mathrm{CDCl}_{3}\right)$.

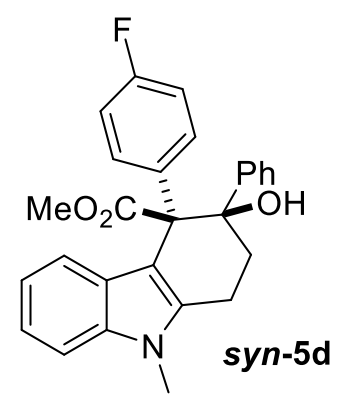

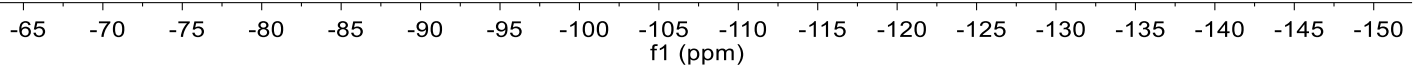

${ }^{19}$ F NMR Spectrum of Compound $\mathbf{5 d}\left(376 \mathrm{MHz}, \mathrm{CDCl}_{3}\right)$. 

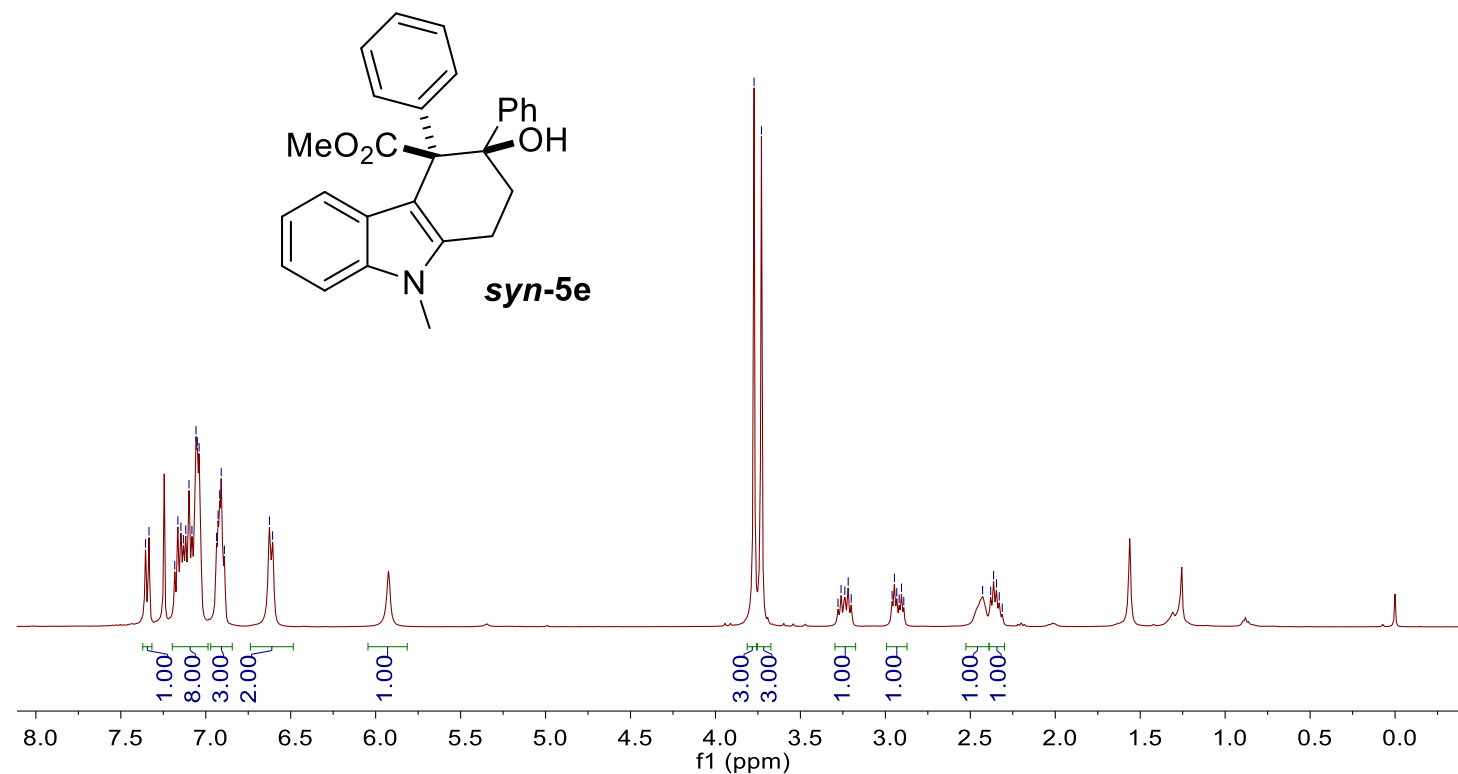

${ }^{1} \mathrm{H}$ NMR Spectrum of Compound $\mathbf{5 e}\left(400 \mathrm{MHz}, \mathrm{CDCl}_{3}\right)$.

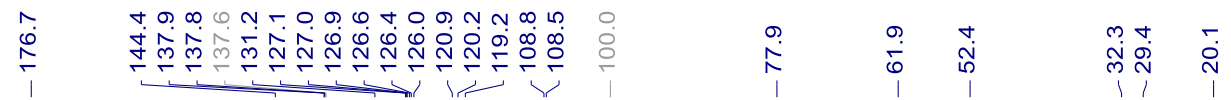

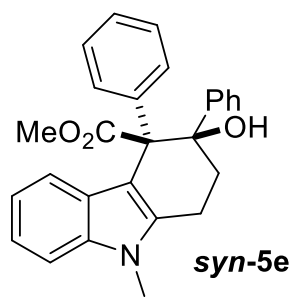

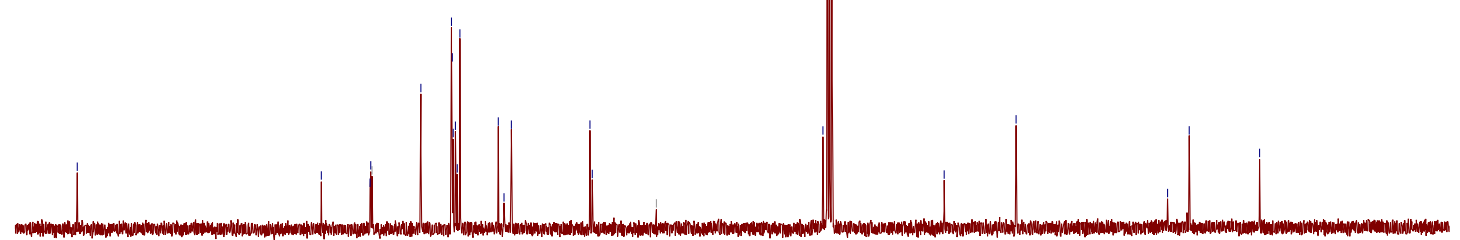

$\begin{array}{lllllllllllllllllll}180 & 170 & 160 & 150 & 140 & 130 & 120 & 110 & 100 \underset{\mathrm{f} 1(\mathrm{ppm})}{90} 80 & 70 & 60 & 50 & 40 & 30 & 20 & 10 & 0\end{array}$

${ }^{13} \mathrm{C}$ NMR Spectrum of Compound $\mathbf{5 e}\left(100 \mathrm{MHz}, \mathrm{CDCl}_{3}\right)$. 


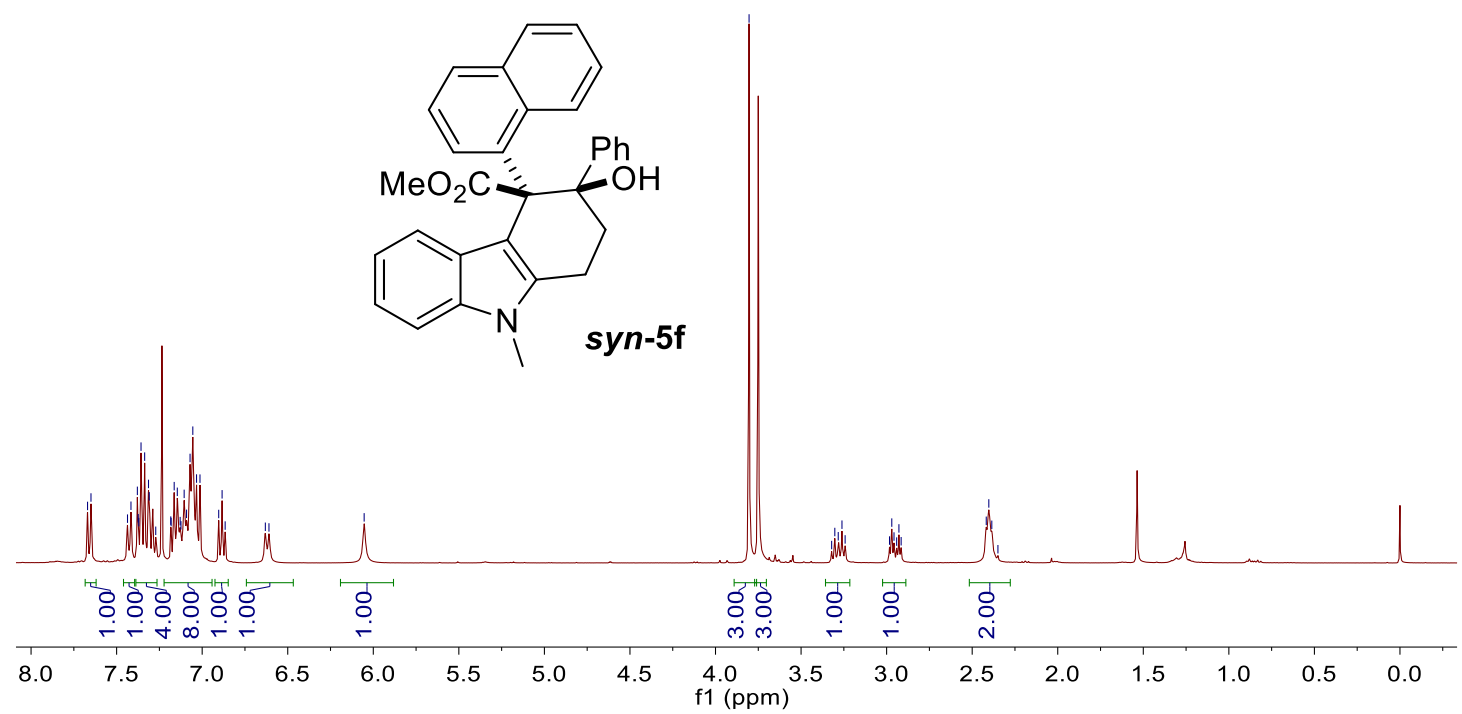

${ }^{1} \mathrm{H}$ NMR Spectrum of Compound $\mathbf{5 f}\left(400 \mathrm{MHz}, \mathrm{CDCl}_{3}\right)$.

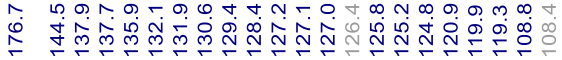

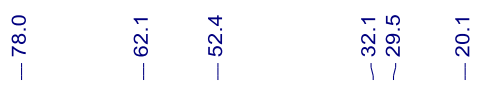
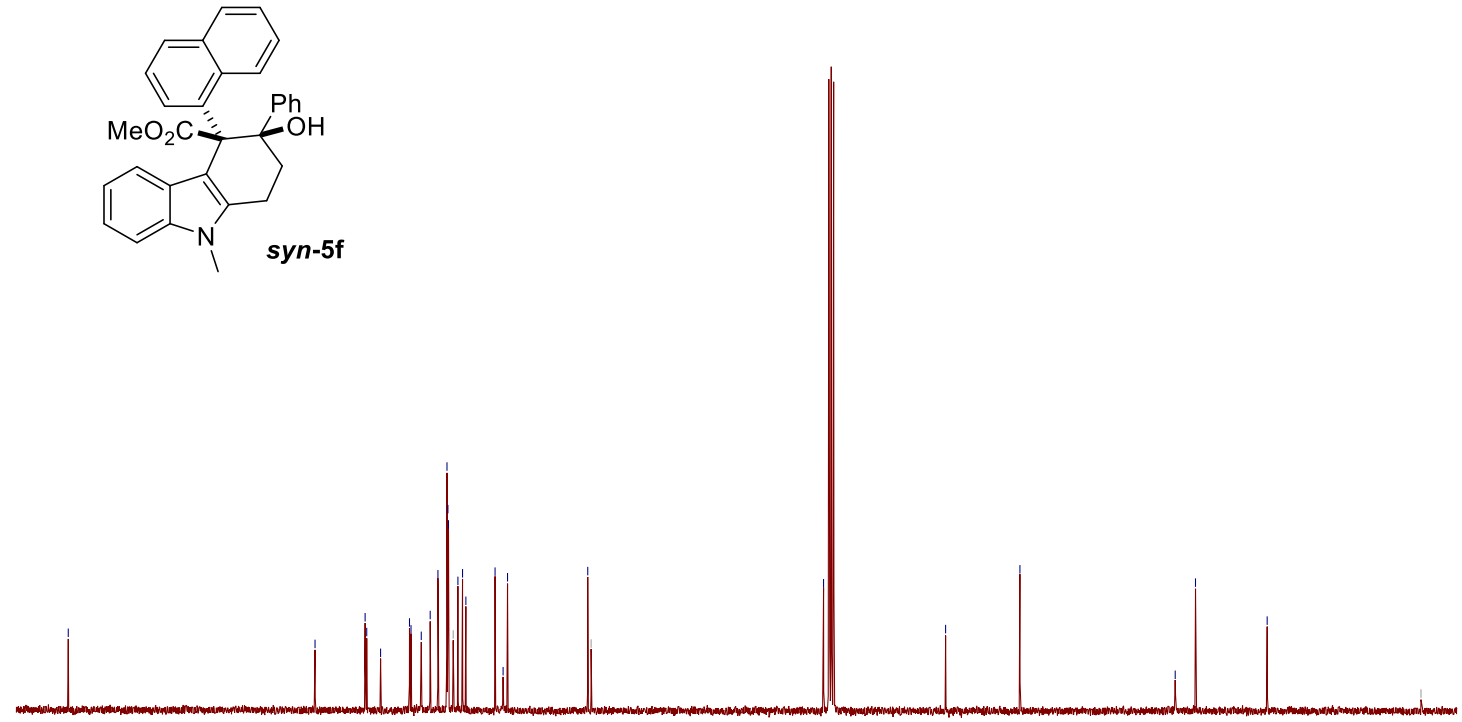

180

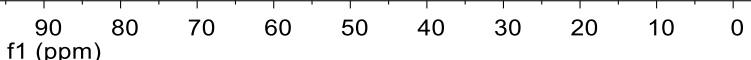

${ }^{13} \mathrm{C}$ NMR Spectrum of Compound $\mathbf{5 f}\left(100 \mathrm{MHz}, \mathrm{CDCl}_{3}\right)$. 
<smiles>COC(=O)[C@]1(c2cccc(Cl)c2)c2c(n(C)c3ccccc23)CC[C@]1(O)c1ccccc1</smiles>

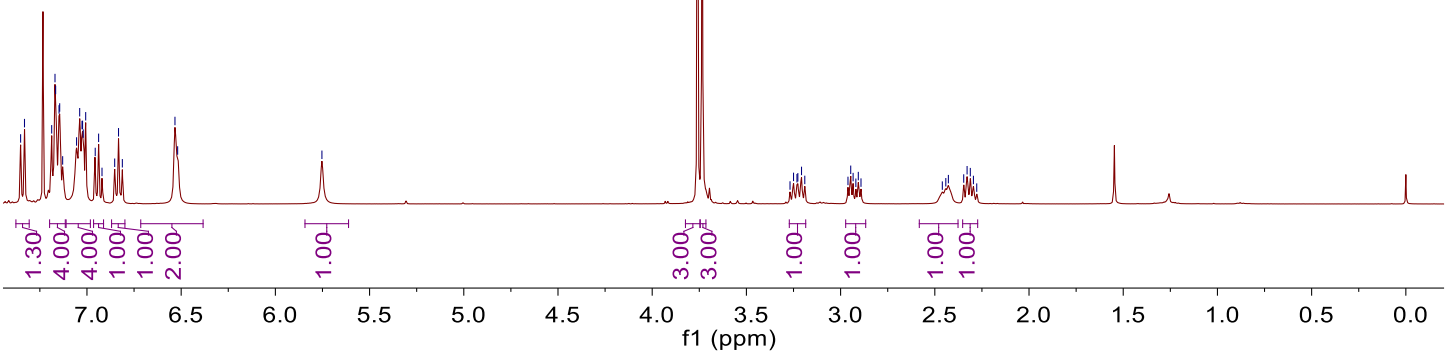

${ }^{1} \mathrm{H}$ NMR Spectrum of Compound $\mathbf{5 g}\left(400 \mathrm{MHz}, \mathrm{CDCl}_{3}\right)$.

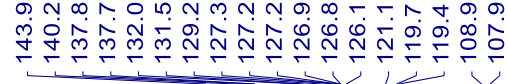

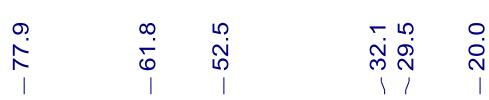

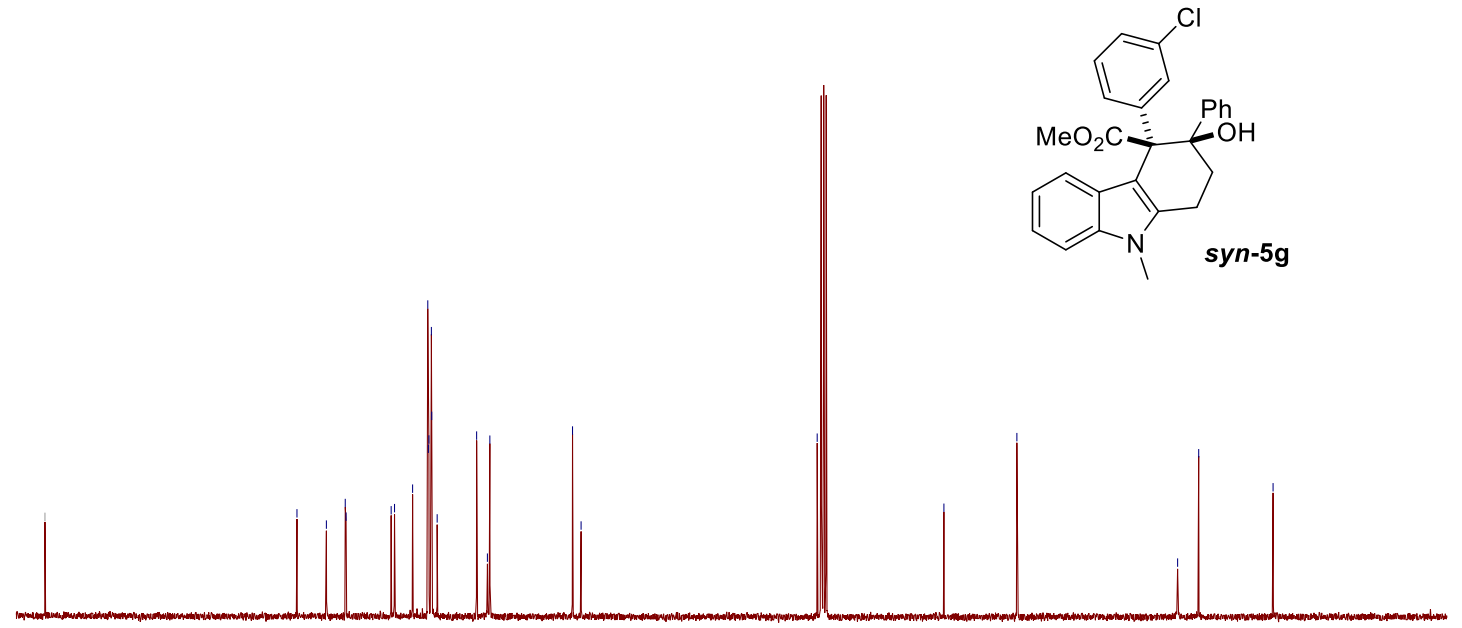

$\begin{array}{llllllllllllllllll}170 & 160 & 150 & 140 & 130 & 120 & 110 & 100 & \underset{\mathrm{f} 1}{90}(\mathrm{ppm}) & 80 & 70 & 60 & 50 & 40 & 30 & 20 & 10 & 0\end{array}$

${ }^{13} \mathrm{C}$ NMR Spectrum of Compound $\mathbf{5 g}\left(100 \mathrm{MHz}, \mathrm{CDCl}_{3}\right)$. 


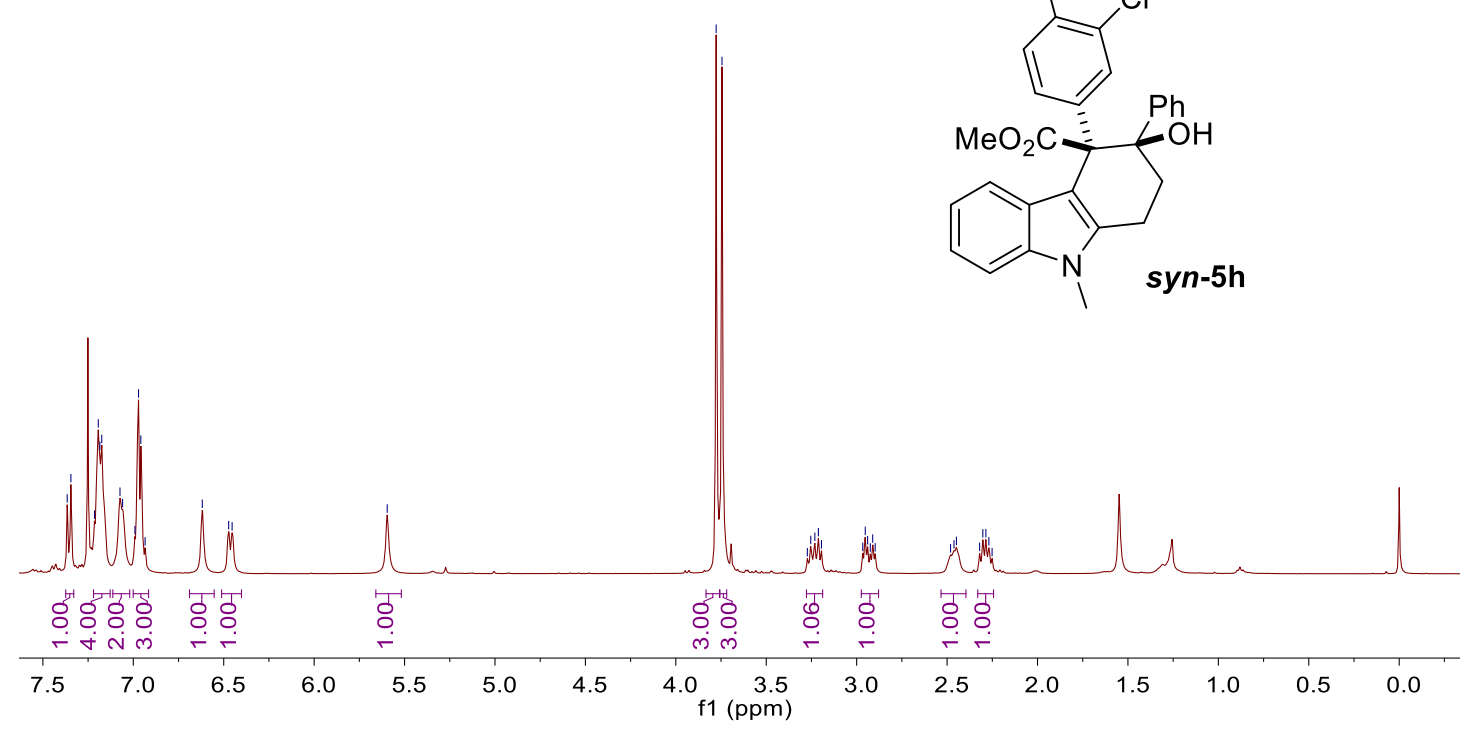

${ }^{1} \mathrm{H}$ NMR Spectrum of Compound $\mathbf{5 h}\left(400 \mathrm{MHz}, \mathrm{CDCl}_{3}\right)$.

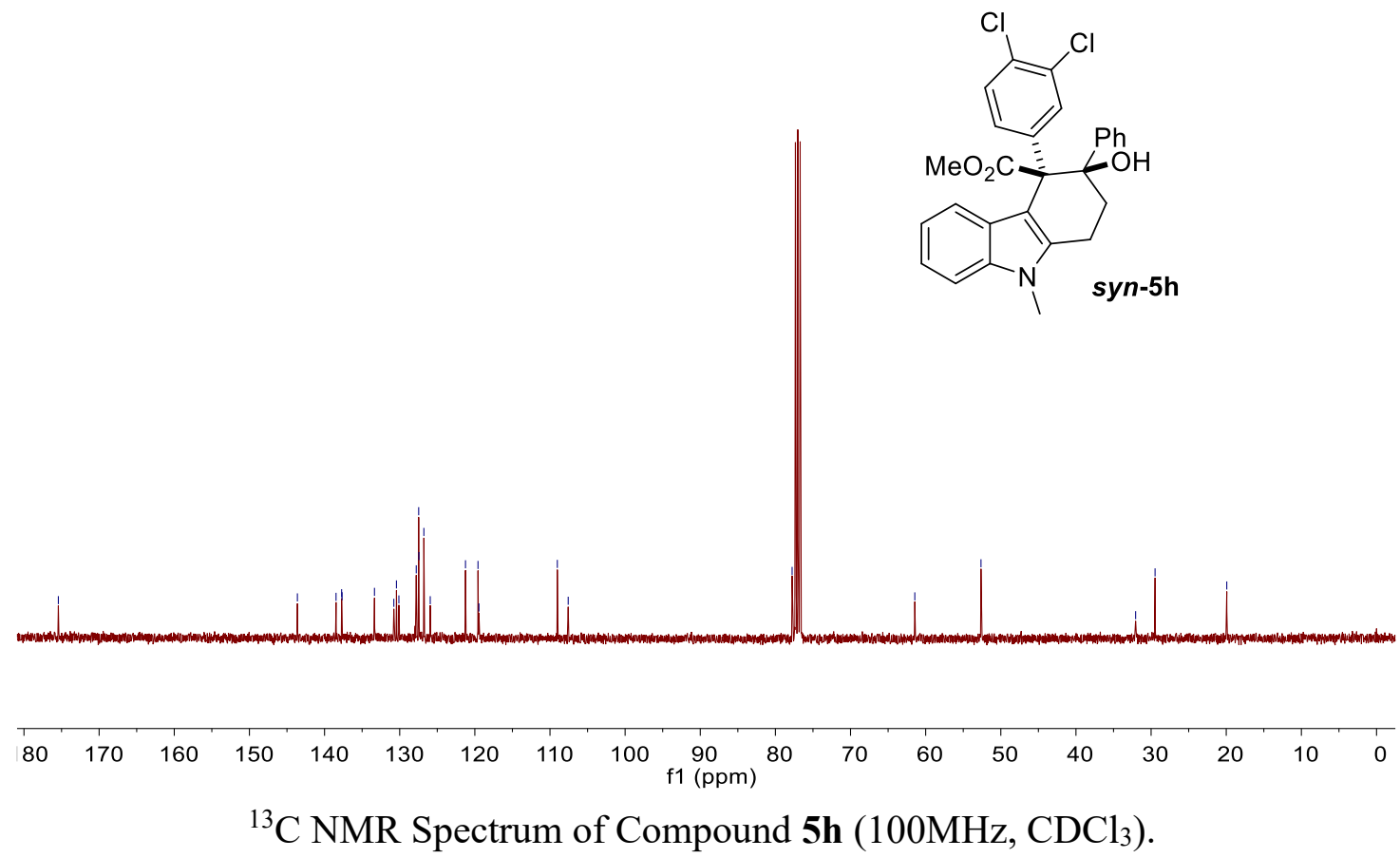



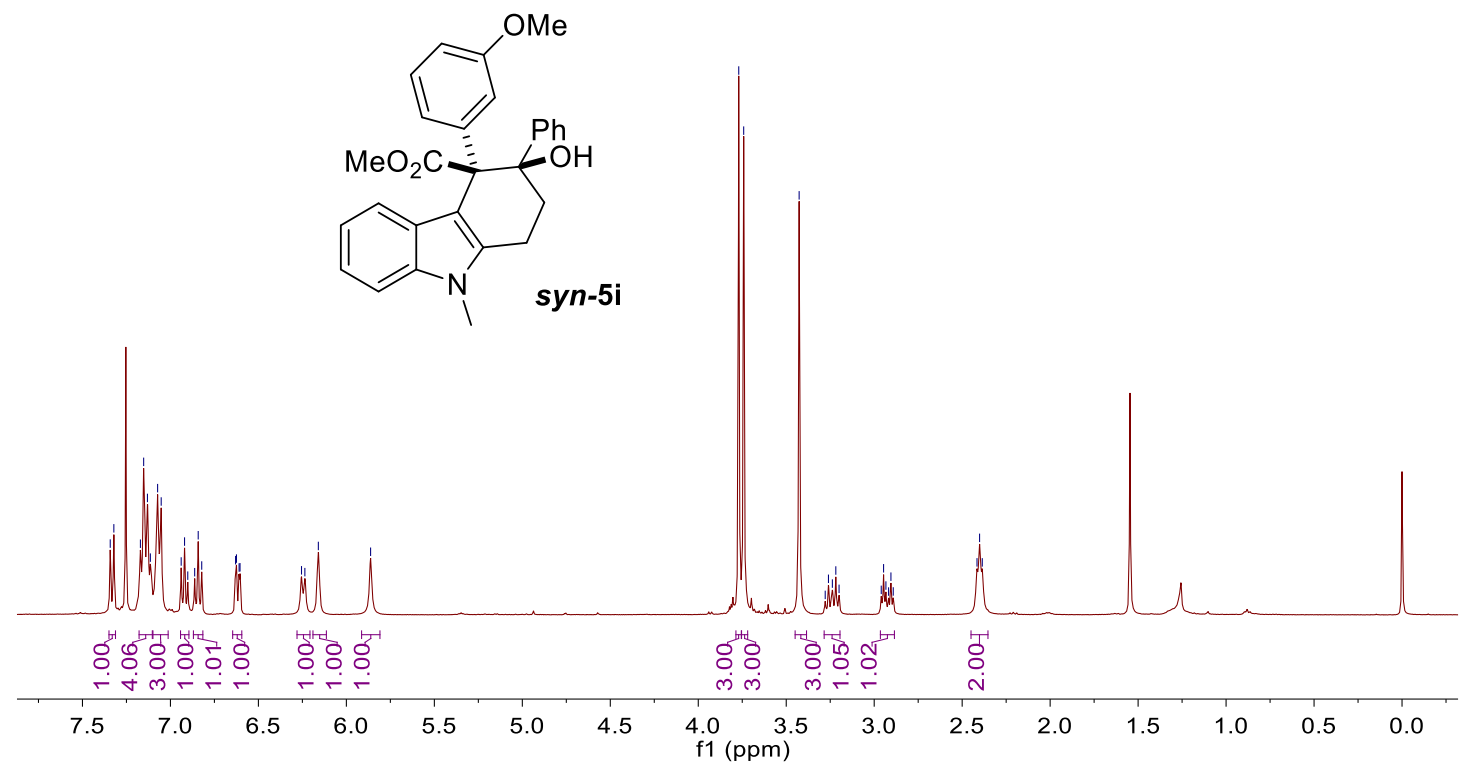

${ }^{1} \mathrm{H}$ NMR Spectrum of Compound $5 \mathbf{i}\left(400 \mathrm{MHz}, \mathrm{CDCl}_{3}\right)$.

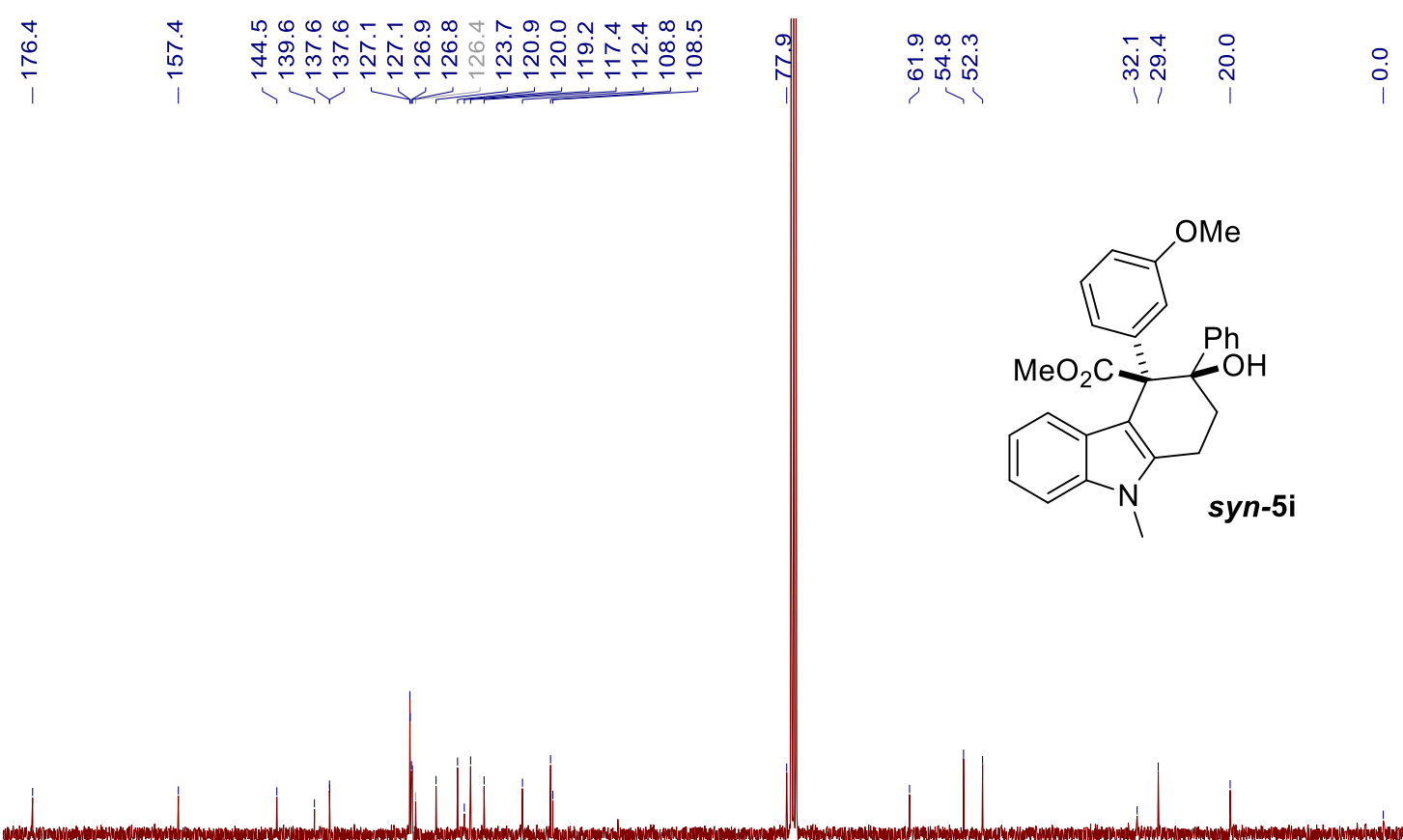

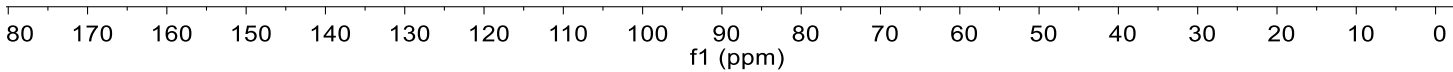

${ }^{13} \mathrm{C}$ NMR Spectrum of Compound $5 \mathbf{i}\left(100 \mathrm{MHz}, \mathrm{CDCl}_{3}\right)$. 

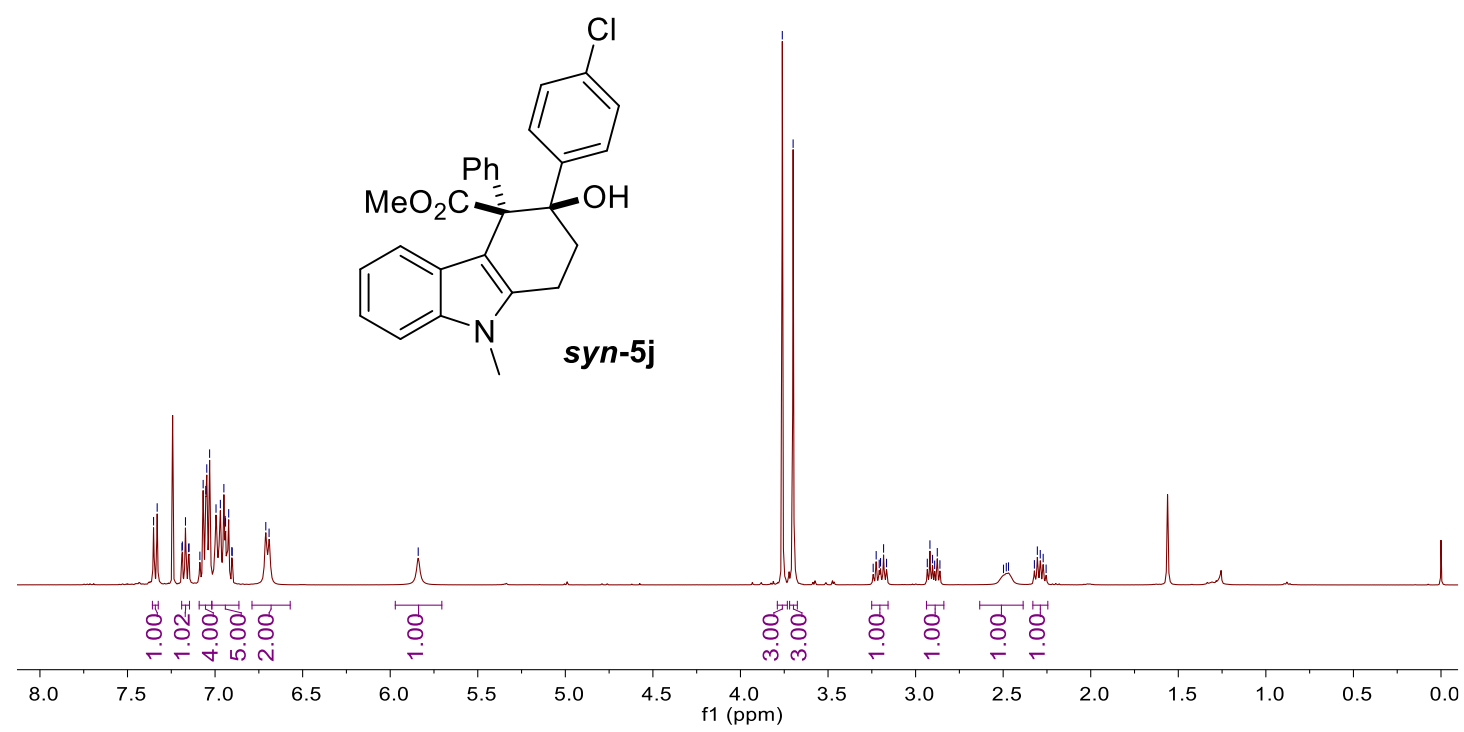

${ }^{1} \mathrm{H}$ NMR Spectrum of Compound $\mathbf{5 j}\left(400 \mathrm{MHz}, \mathrm{CDCl}_{3}\right)$.

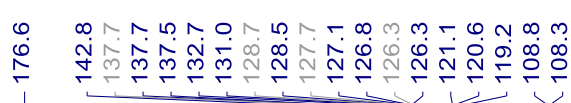

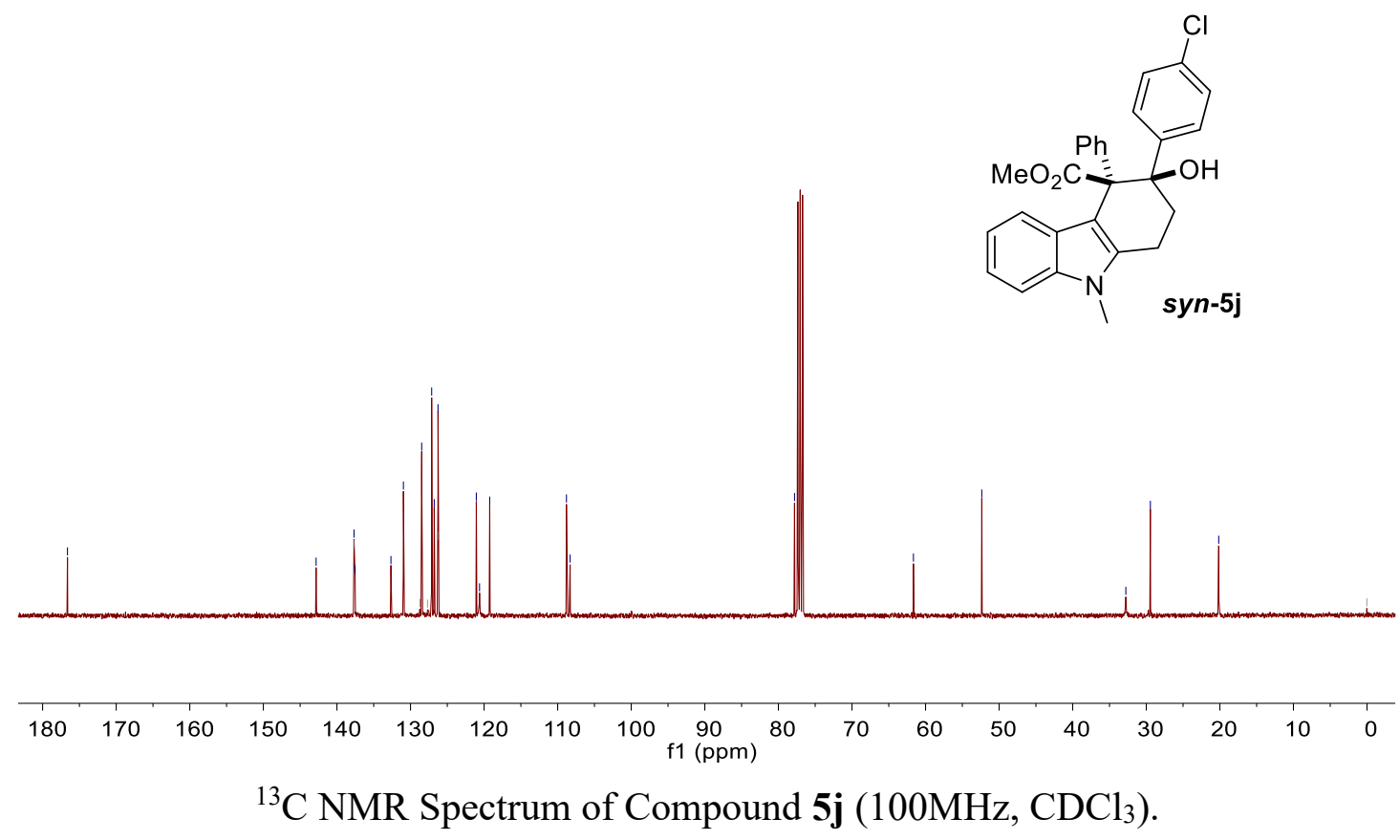



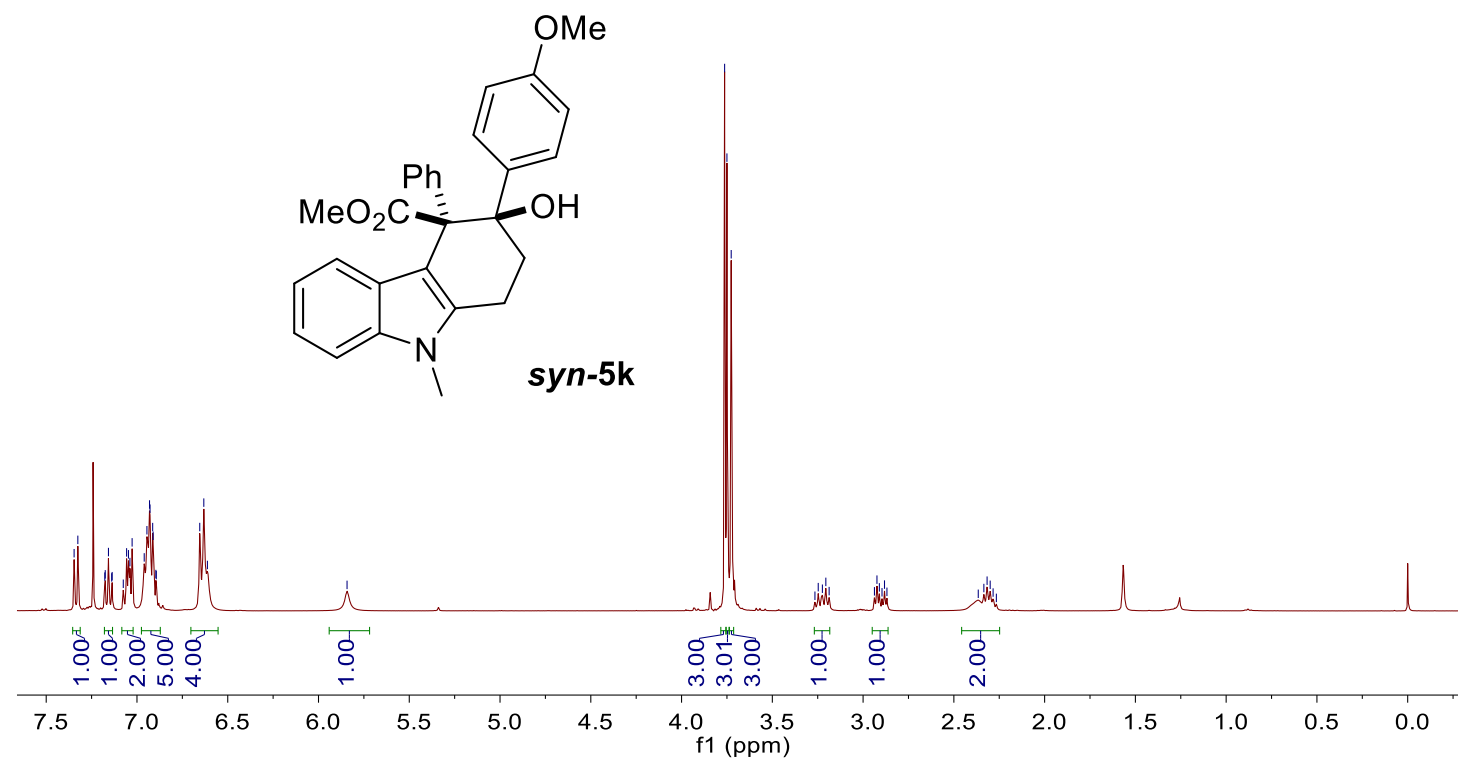

${ }^{1} \mathrm{H}$ NMR Spectrum of Compound $\mathbf{5 k}\left(400 \mathrm{MHz}, \mathrm{CDCl}_{3}\right)$.
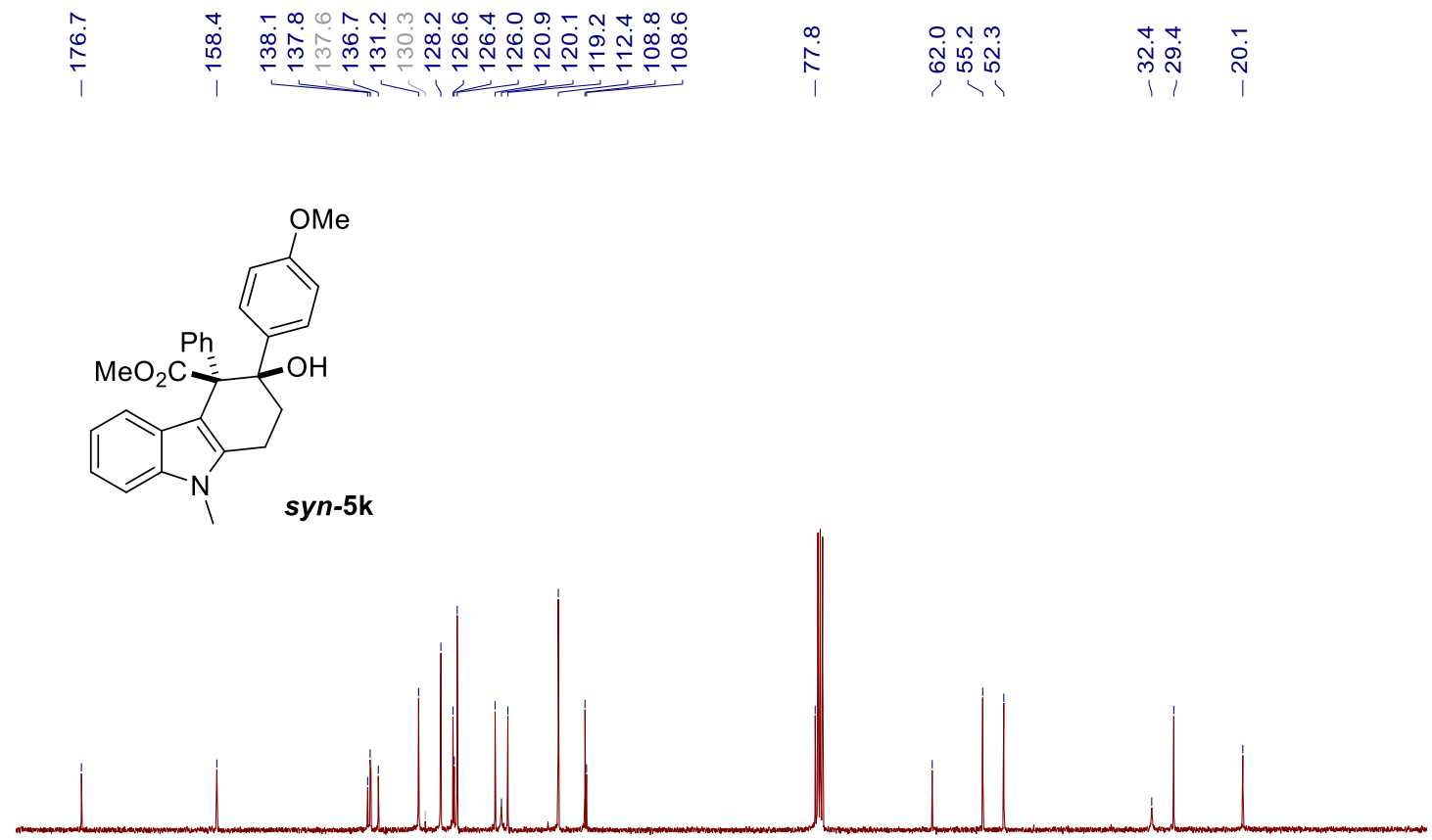

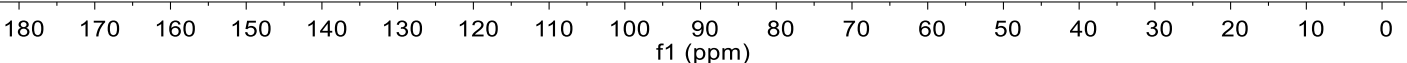

${ }^{13} \mathrm{C}$ NMR Spectrum of Compound $\mathbf{5 k}\left(100 \mathrm{MHz}, \mathrm{CDCl}_{3}\right)$. 


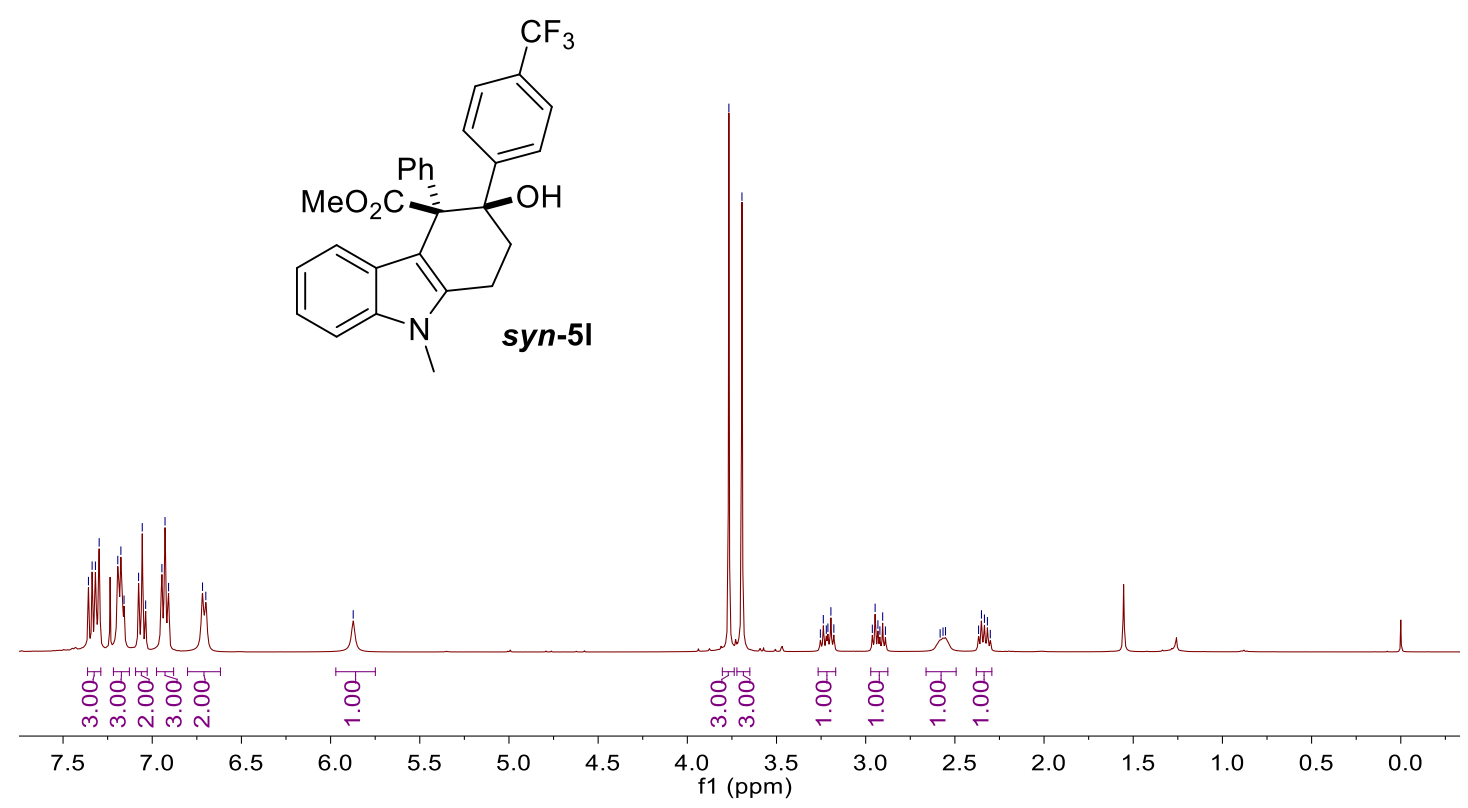

${ }^{1} \mathrm{H}$ NMR Spectrum of Compound $\mathbf{5 l}\left(400 \mathrm{MHz}, \mathrm{CDCl}_{3}\right)$.

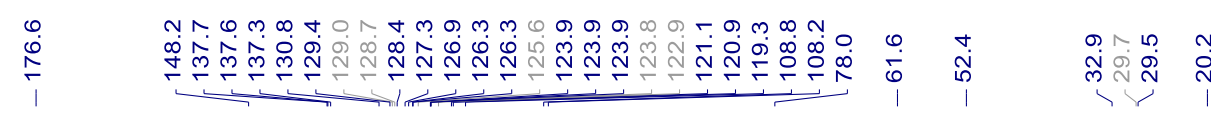

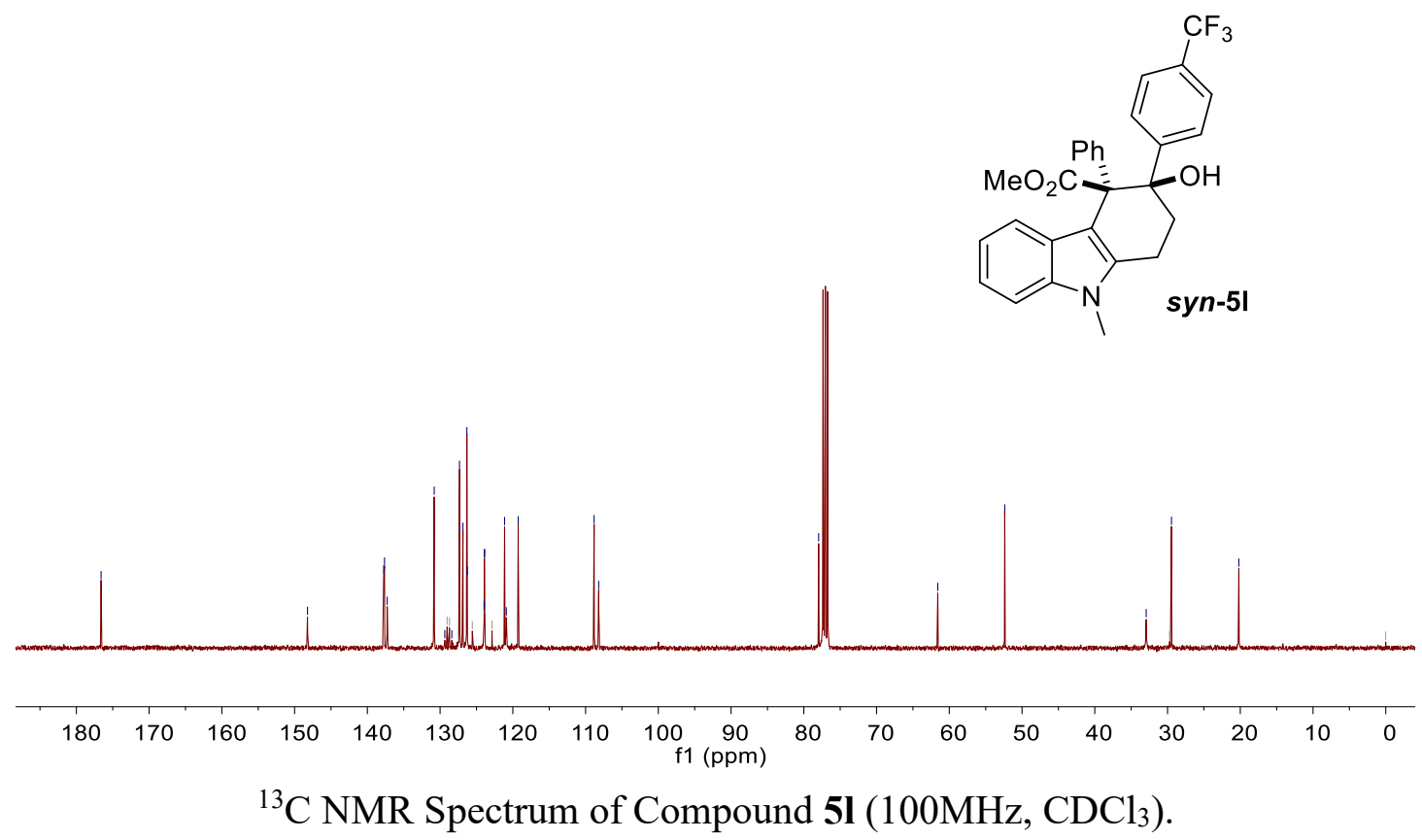



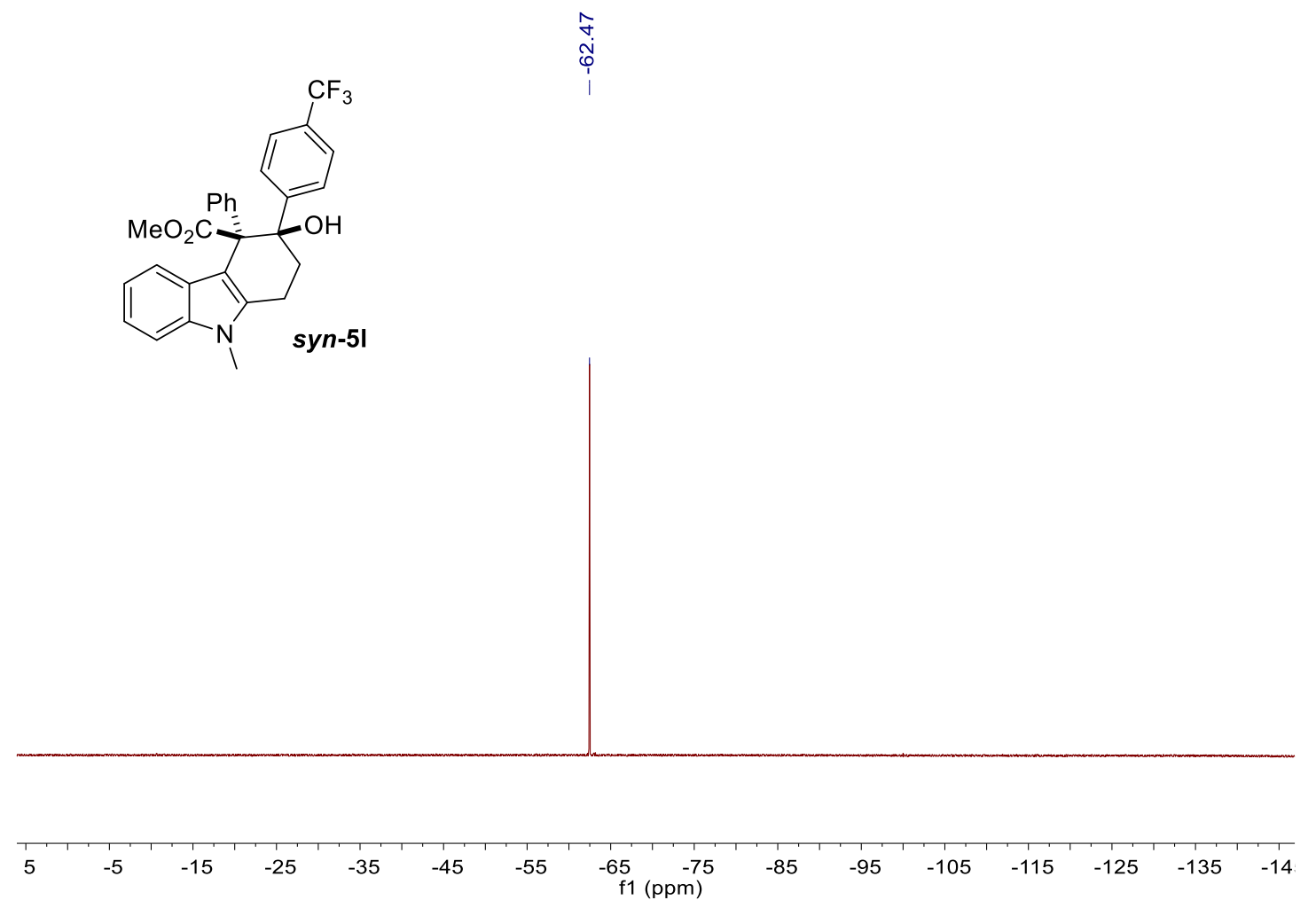

${ }^{19}$ F NMR Spectrum of Compound $\mathbf{5 l}\left(376 \mathrm{MHz}, \mathrm{CDCl}_{3}\right)$.

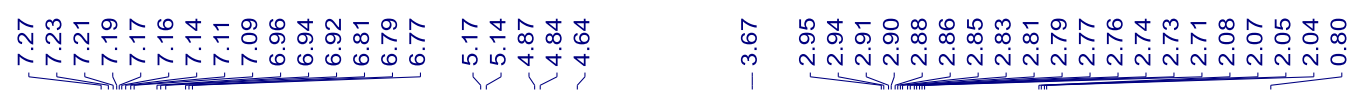

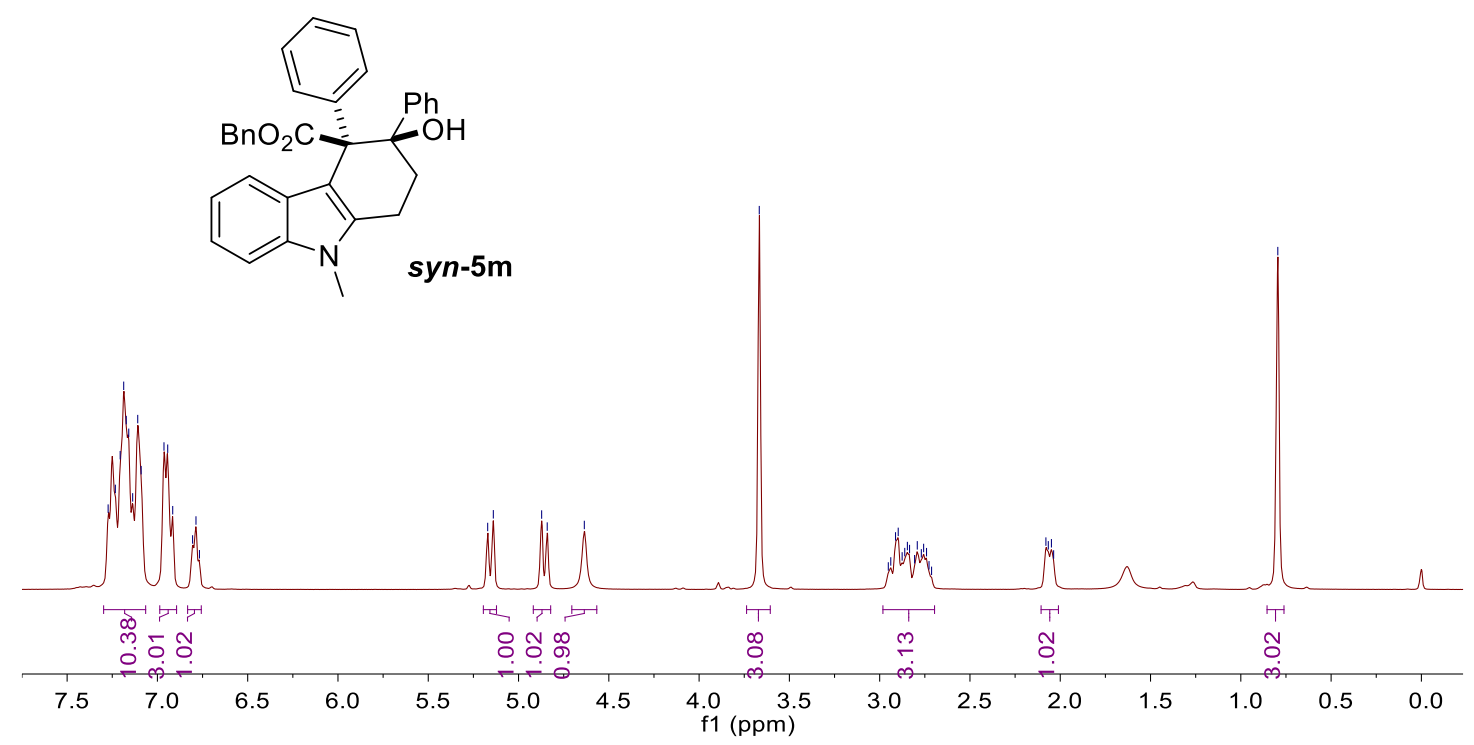

${ }^{1} \mathrm{H}$ NMR Spectrum of Compound $\mathbf{5 m}\left(400 \mathrm{MHz}, \mathrm{CDCl}_{3}\right)$. 


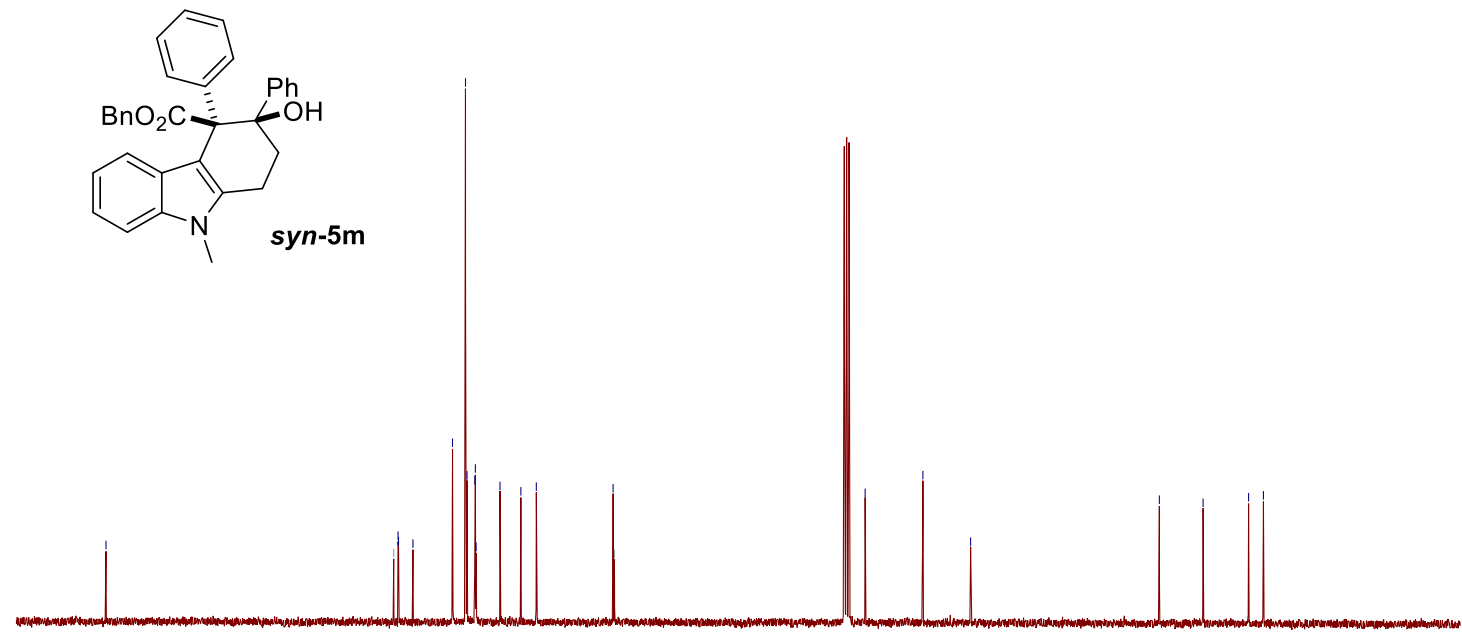

$\begin{array}{lllllllllllllllllll}180 & 170 & 160 & 150 & 140 & 130 & 120 & 110 & 100 & 90 & 80 & 70 & 60 & 50 & 40 & 30 & 20 & 10 & 0\end{array}$

${ }^{13} \mathrm{C}$ NMR Spectrum of Compound $5 \mathbf{m}\left(100 \mathrm{MHz}, \mathrm{CDCl}_{3}\right)$.

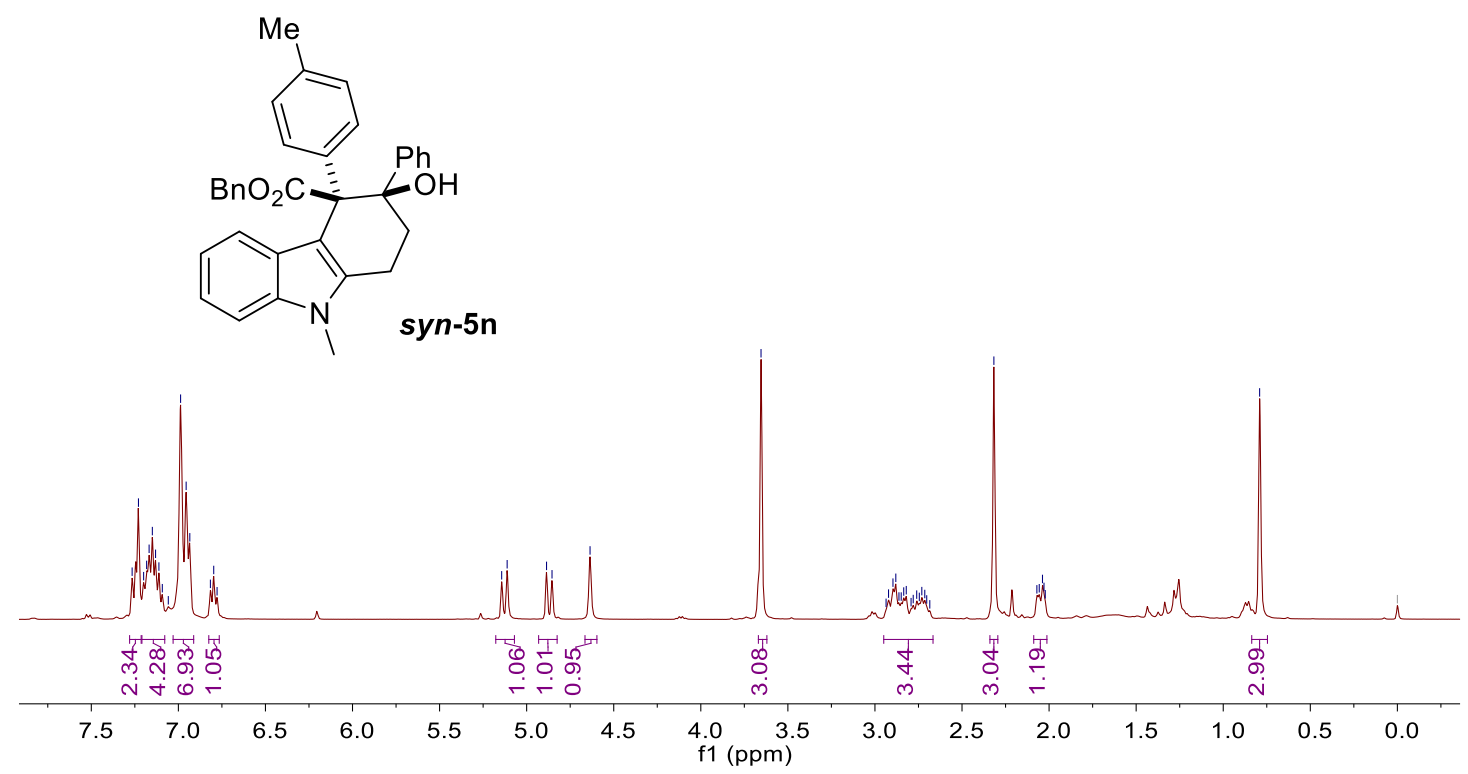

${ }^{1} \mathrm{H}$ NMR Spectrum of Compound 5n $\left(400 \mathrm{MHz}, \mathrm{CDCl}_{3}\right)$. 

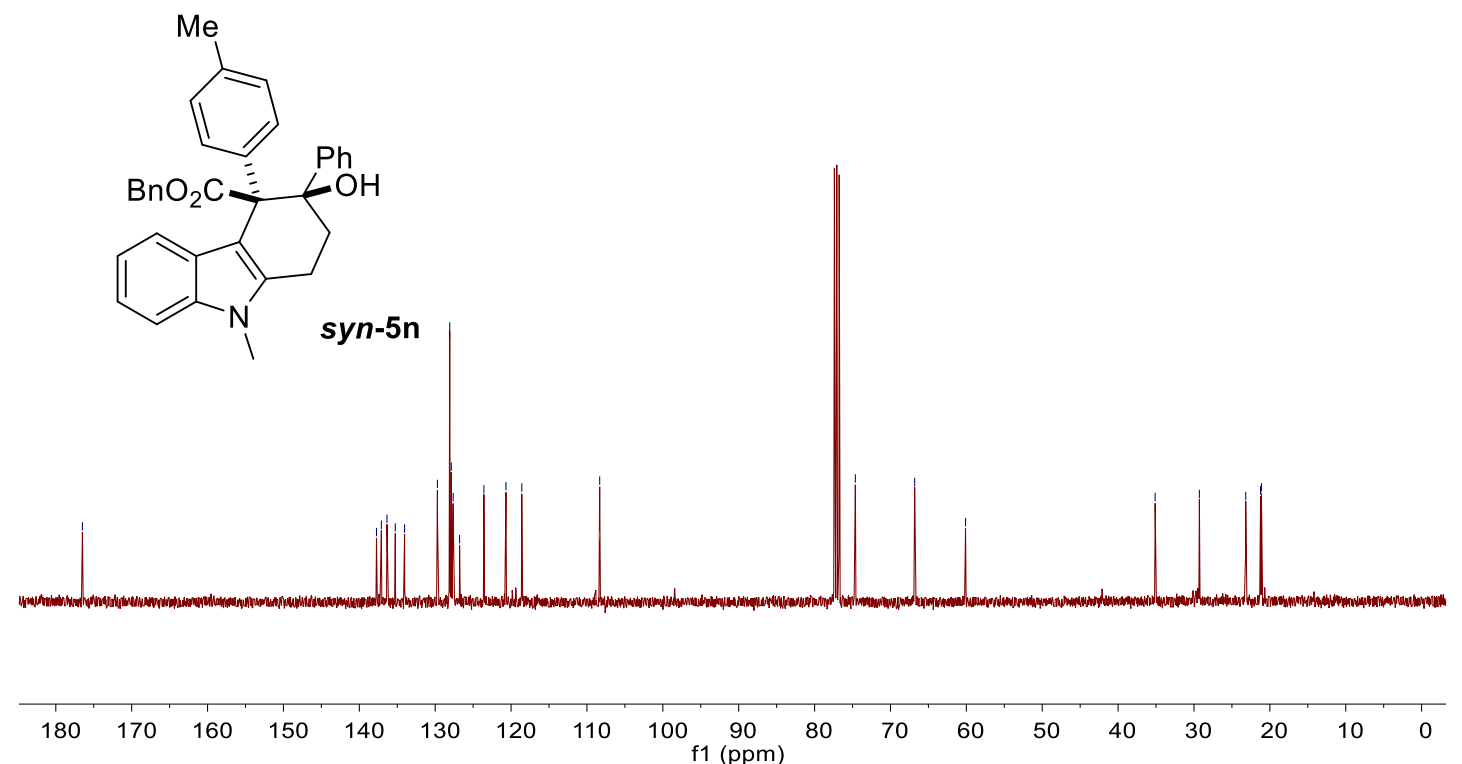

${ }^{13} \mathrm{C}$ NMR Spectrum of Compound $\mathbf{5 n}\left(100 \mathrm{MHz}, \mathrm{CDCl}_{3}\right)$.

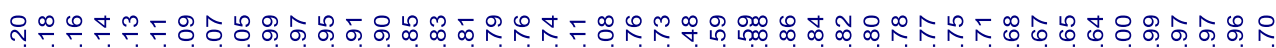

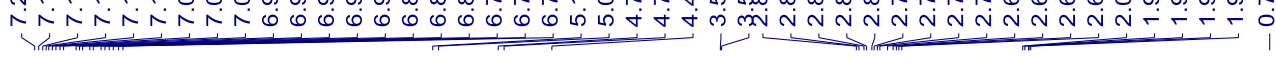

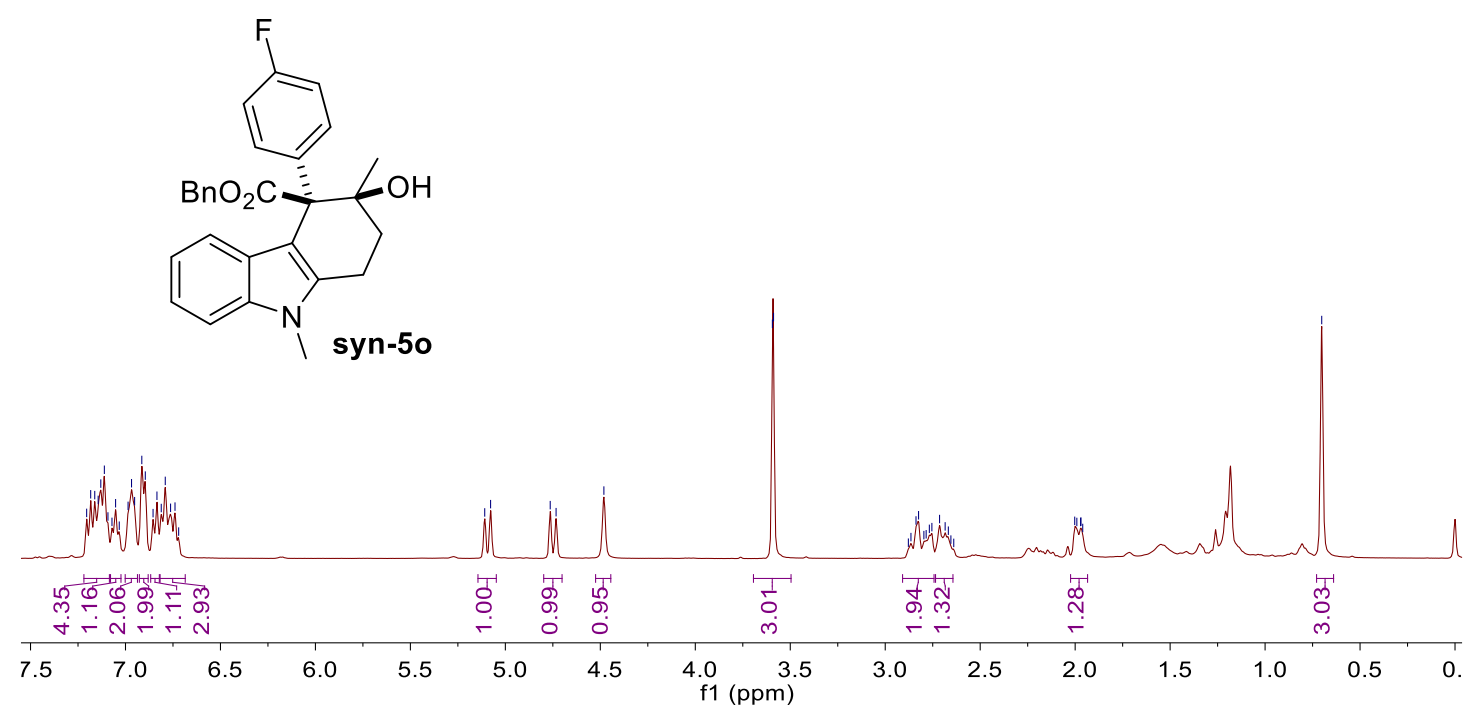

${ }^{1} \mathrm{H}$ NMR Spectrum of Compound $\mathbf{5 0}\left(400 \mathrm{MHz}, \mathrm{CDCl}_{3}\right)$. 

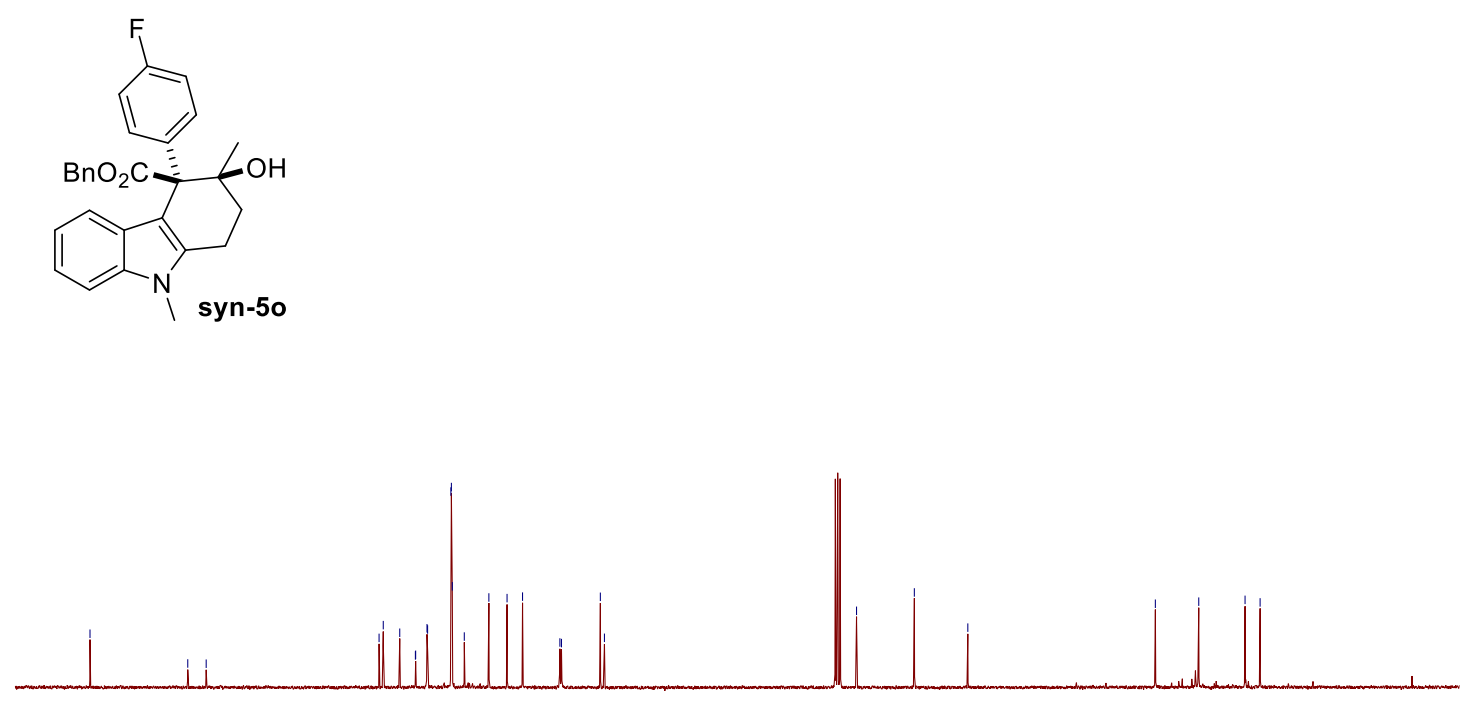

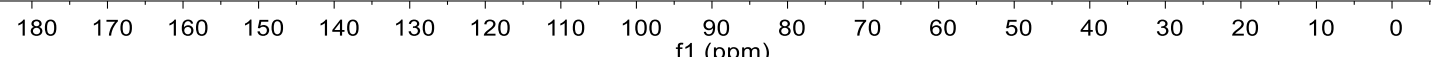
${ }^{13} \mathrm{C}$ NMR Spectrum of Compound $50\left(100 \mathrm{MHz}, \mathrm{CDCl}_{3}\right)$.

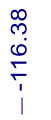<smiles>Cn1c2c(c3ccccc31)[C@](c1ccccc1)(c1ccc(F)cc1)C(C)(O)CC2</smiles>

$\begin{array}{llllllllllllllllllllllllll}10 & 0 & -10 & -20 & -30 & -40 & -50 & -60 & -70 & -80 & -90 & -100 & -110 & -120 & -130 & -140 & -150 & -160 & -170 & -180 & -190 & -200 & -210\end{array}$

${ }^{19} \mathrm{~F}$ NMR Spectrum of Compound $50\left(376 \mathrm{MHz}, \mathrm{CDCl}_{3}\right)$. 


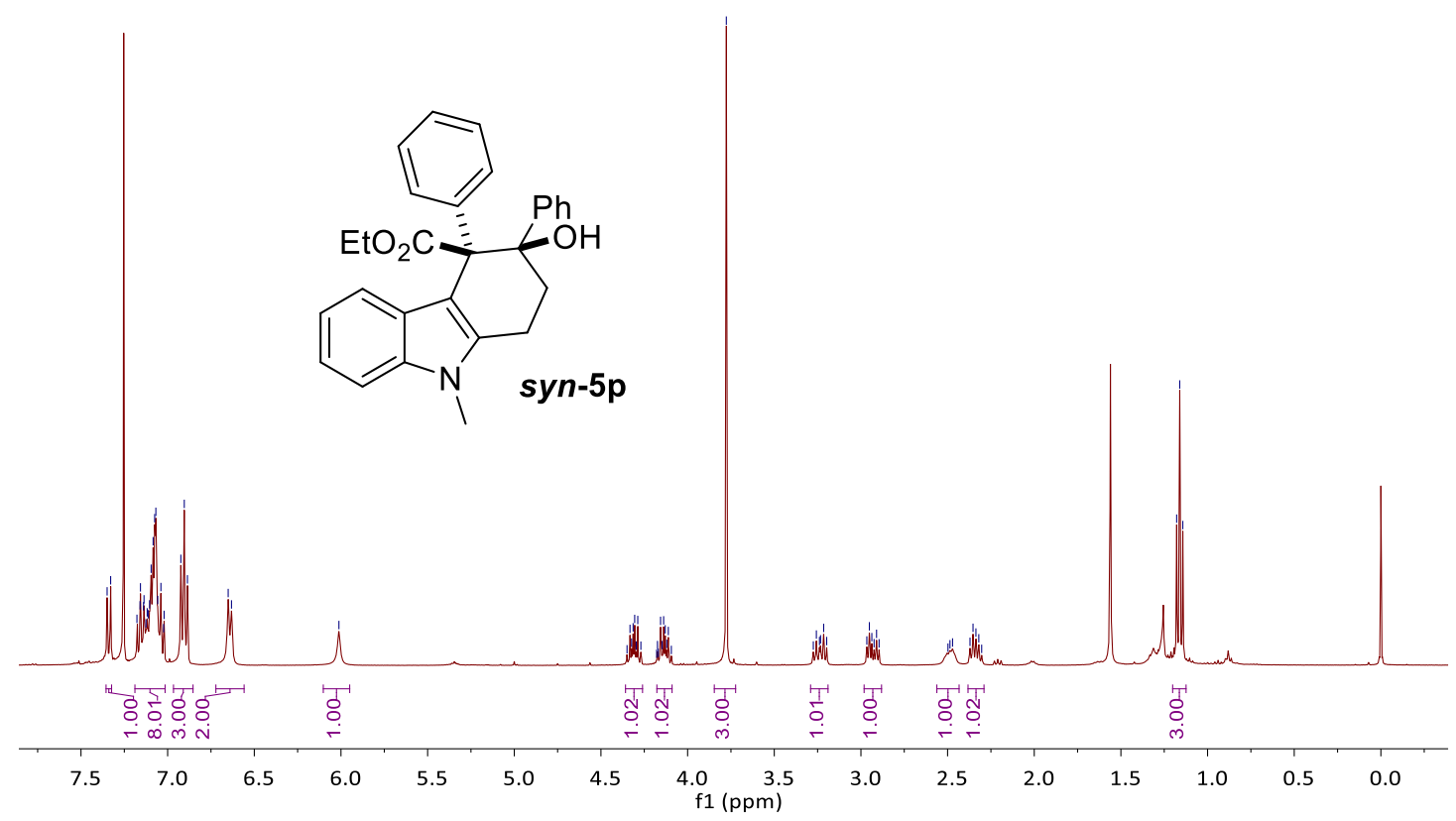

${ }^{1} \mathrm{H}$ NMR Spectrum of Compound $\mathbf{5 p}\left(400 \mathrm{MHz}, \mathrm{CDCl}_{3}\right)$.

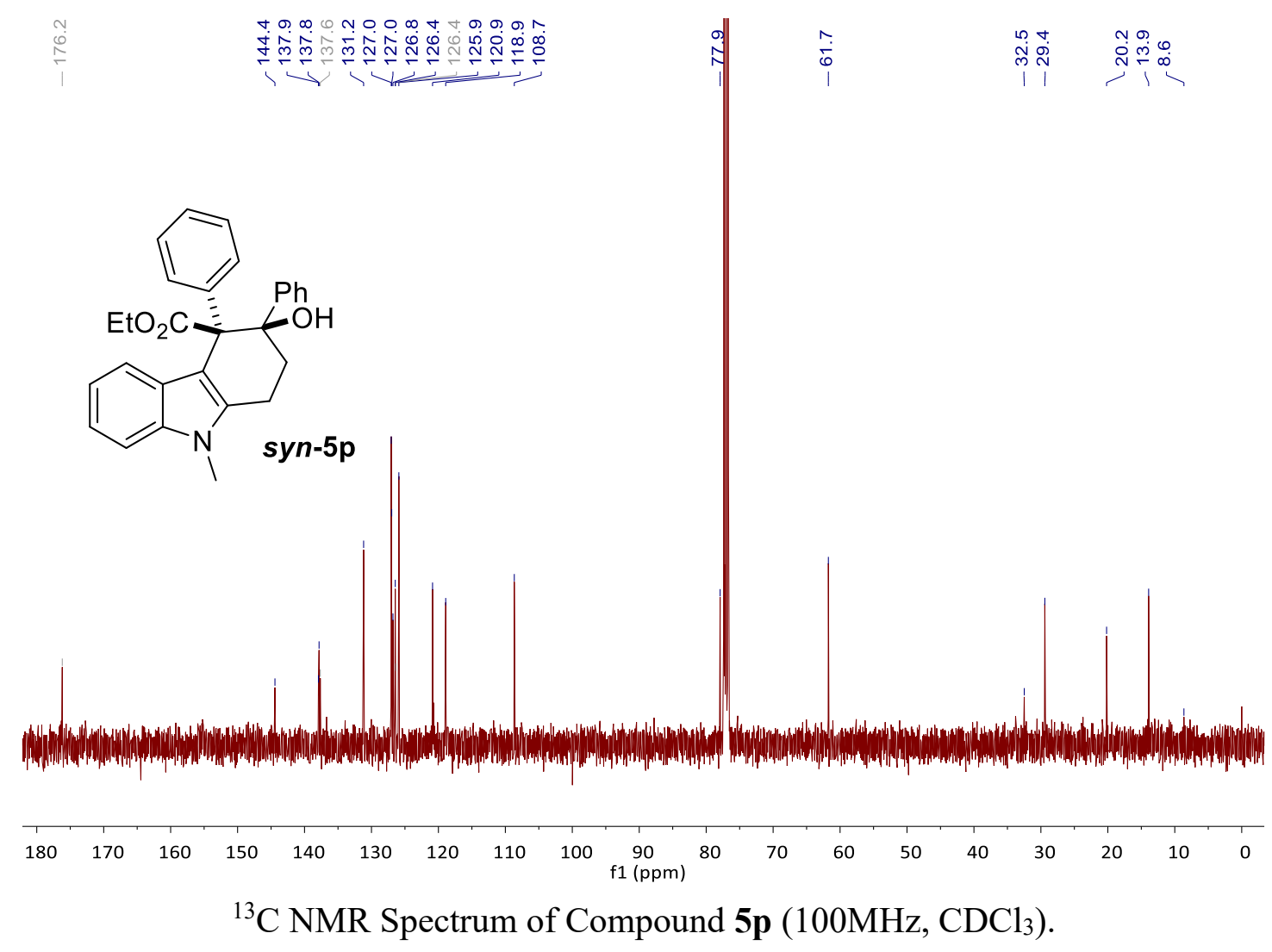




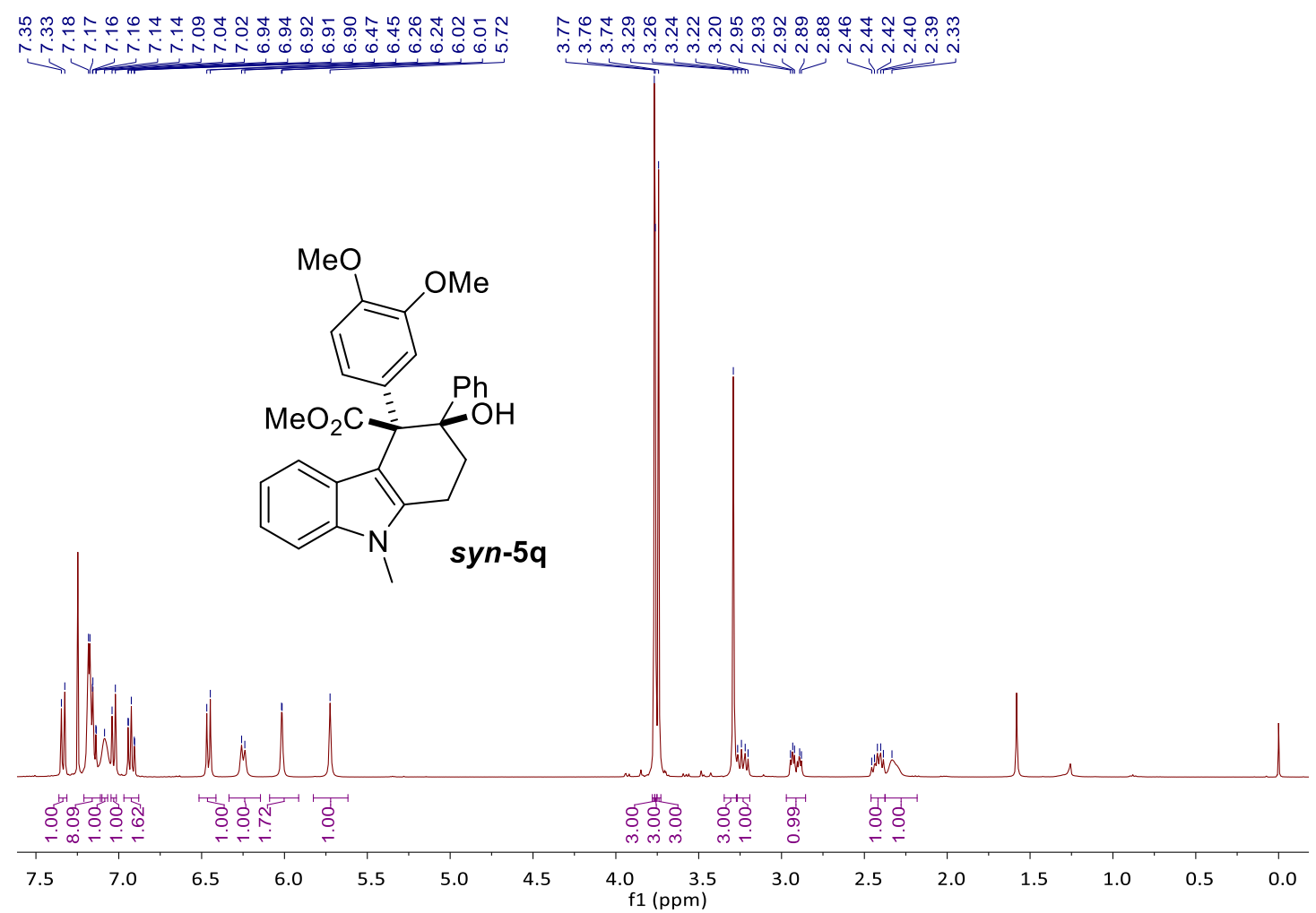

${ }^{1} \mathrm{H}$ NMR Spectrum of Compound $\mathbf{5 q}\left(400 \mathrm{MHz}, \mathrm{CDCl}_{3}\right)$.
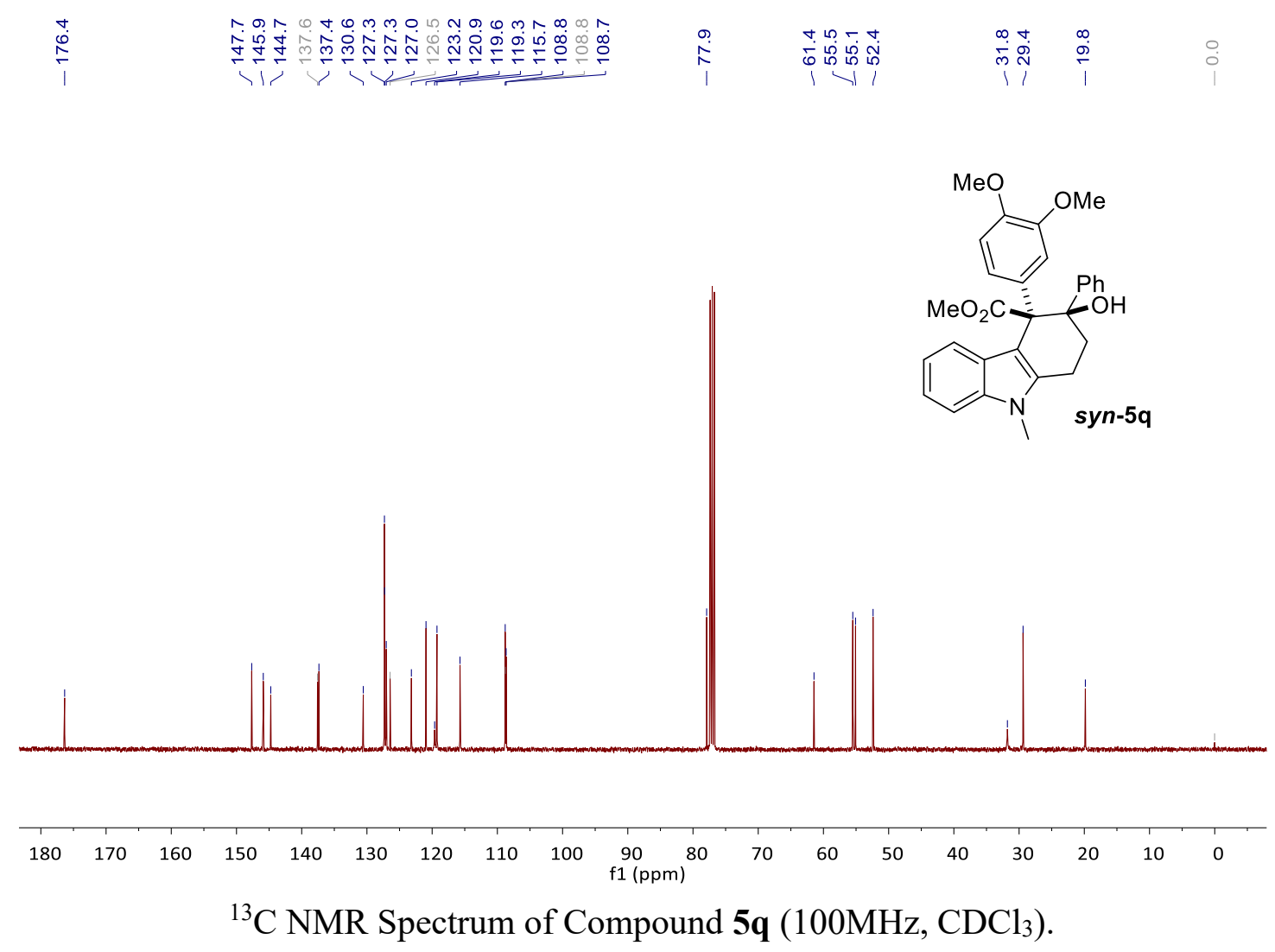


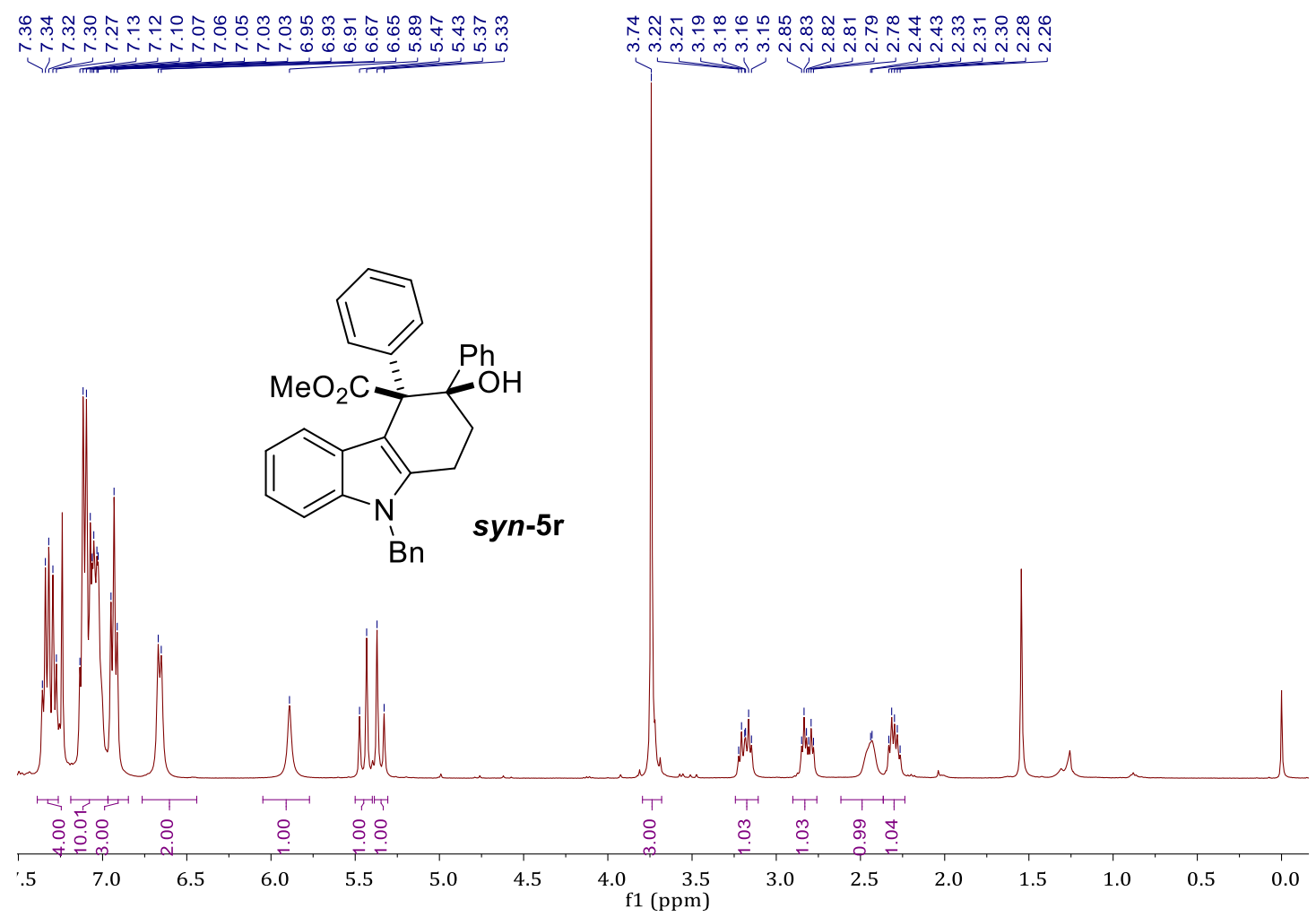

${ }^{1} \mathrm{H}$ NMR Spectrum of Compound $\mathbf{5 r}\left(400 \mathrm{MHz}, \mathrm{CDCl}_{3}\right)$.

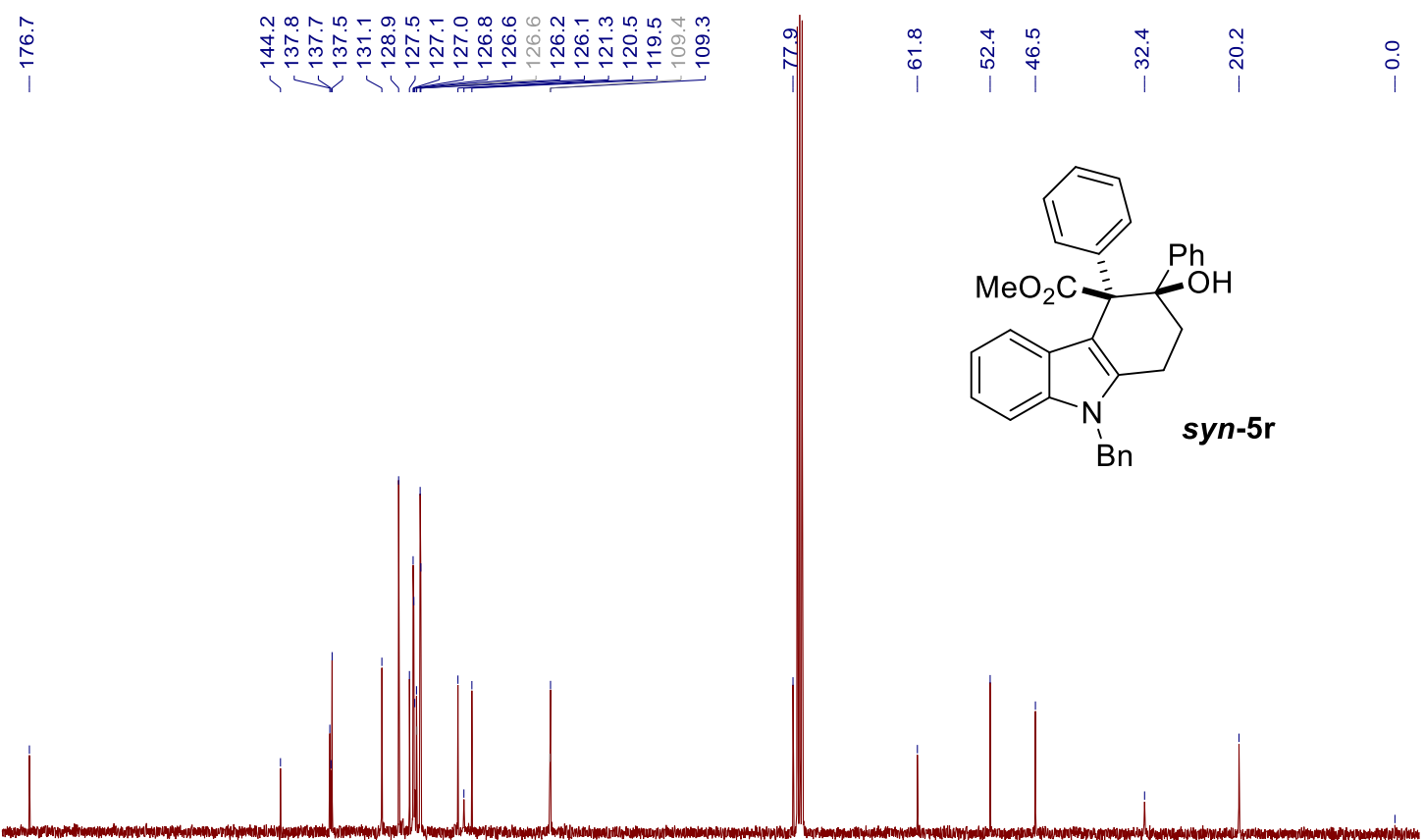

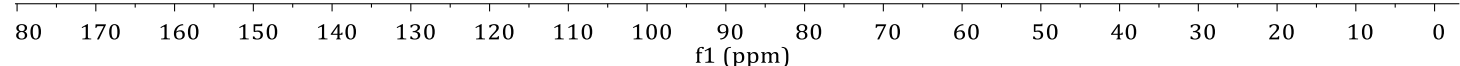

${ }^{13} \mathrm{C}$ NMR Spectrum of Compound $5 \mathbf{r}\left(100 \mathrm{MHz}, \mathrm{CDCl}_{3}\right)$. 

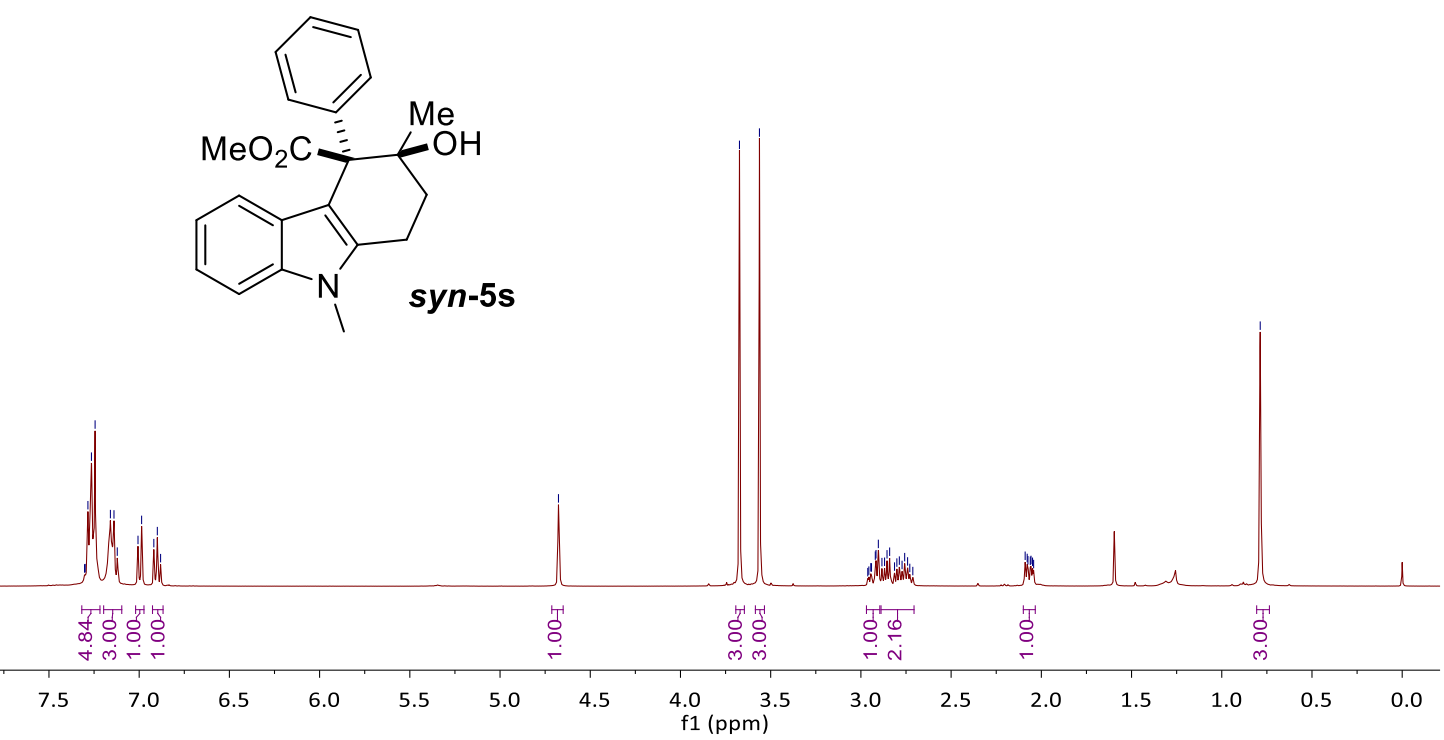

${ }^{1} \mathrm{H}$ NMR Spectrum of Compound $5 \mathbf{s}\left(400 \mathrm{MHz}, \mathrm{CDCl}_{3}\right)$.
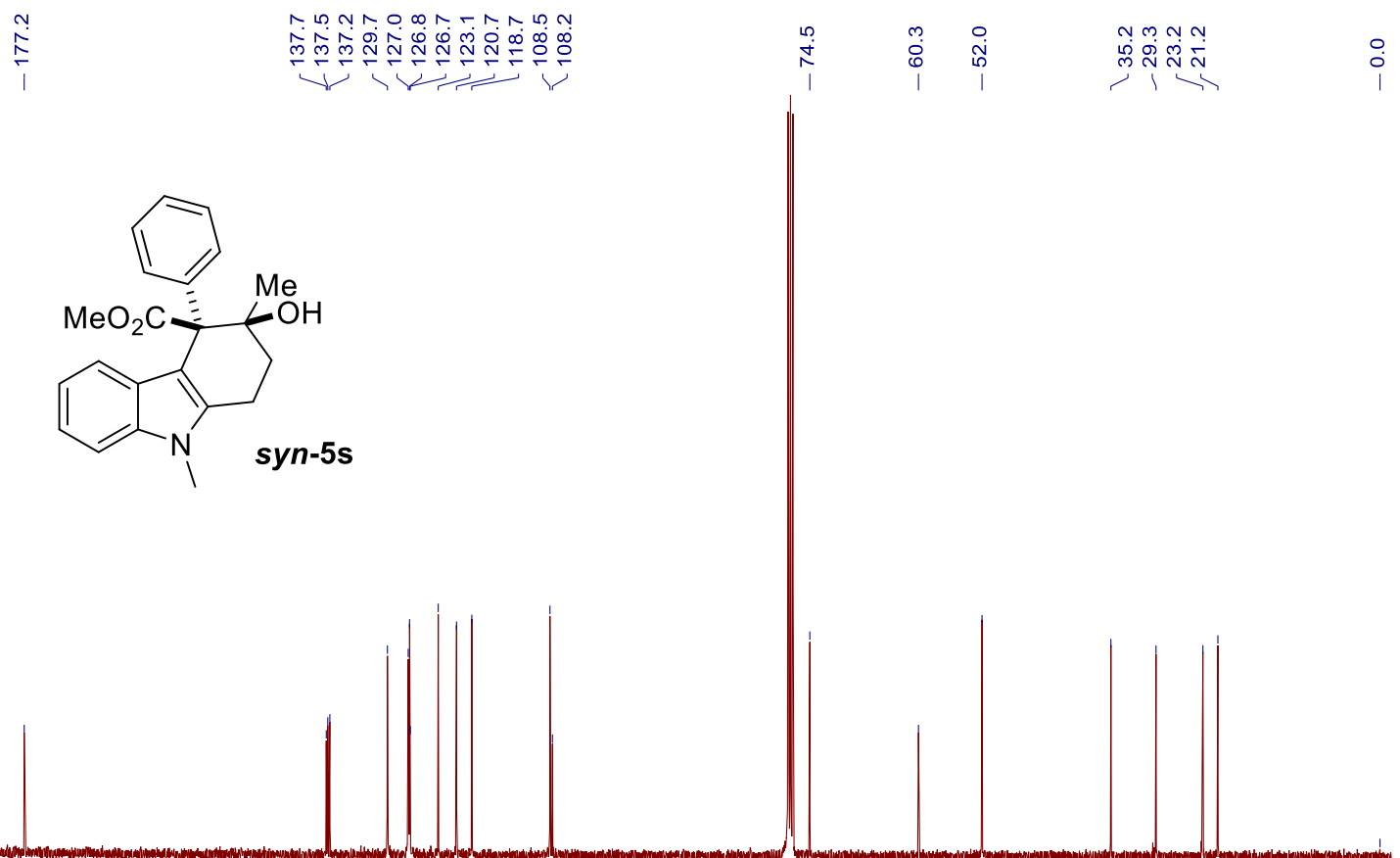

180

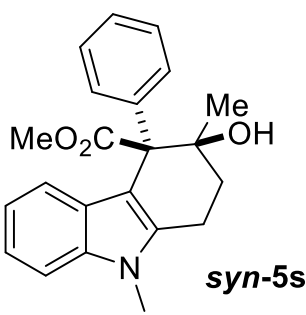

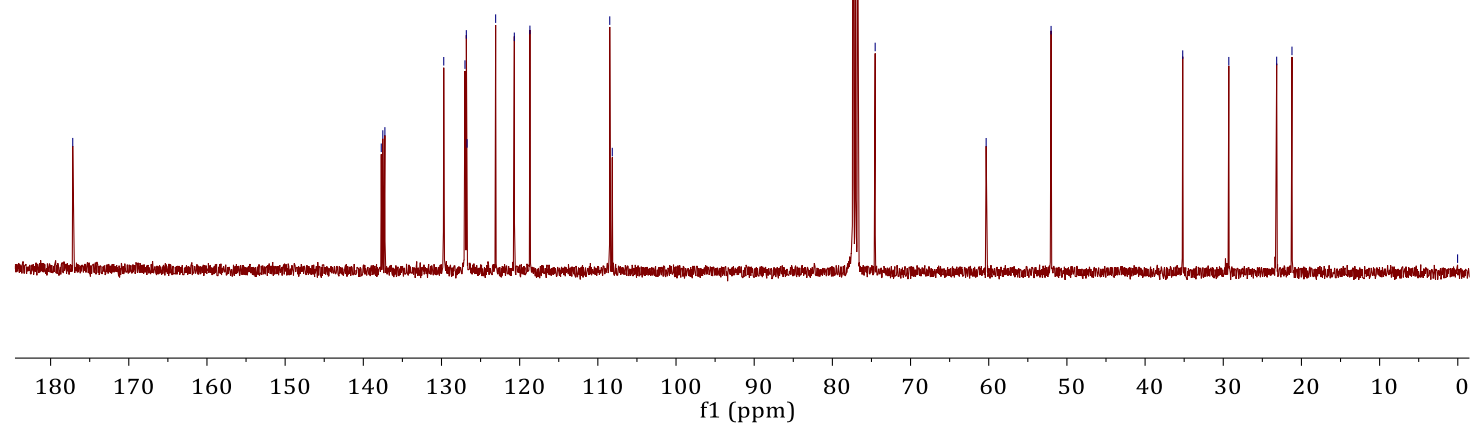

${ }^{13} \mathrm{C}$ NMR Spectrum of Compound $5 \mathbf{s}\left(100 \mathrm{MHz}, \mathrm{CDCl}_{3}\right)$. 


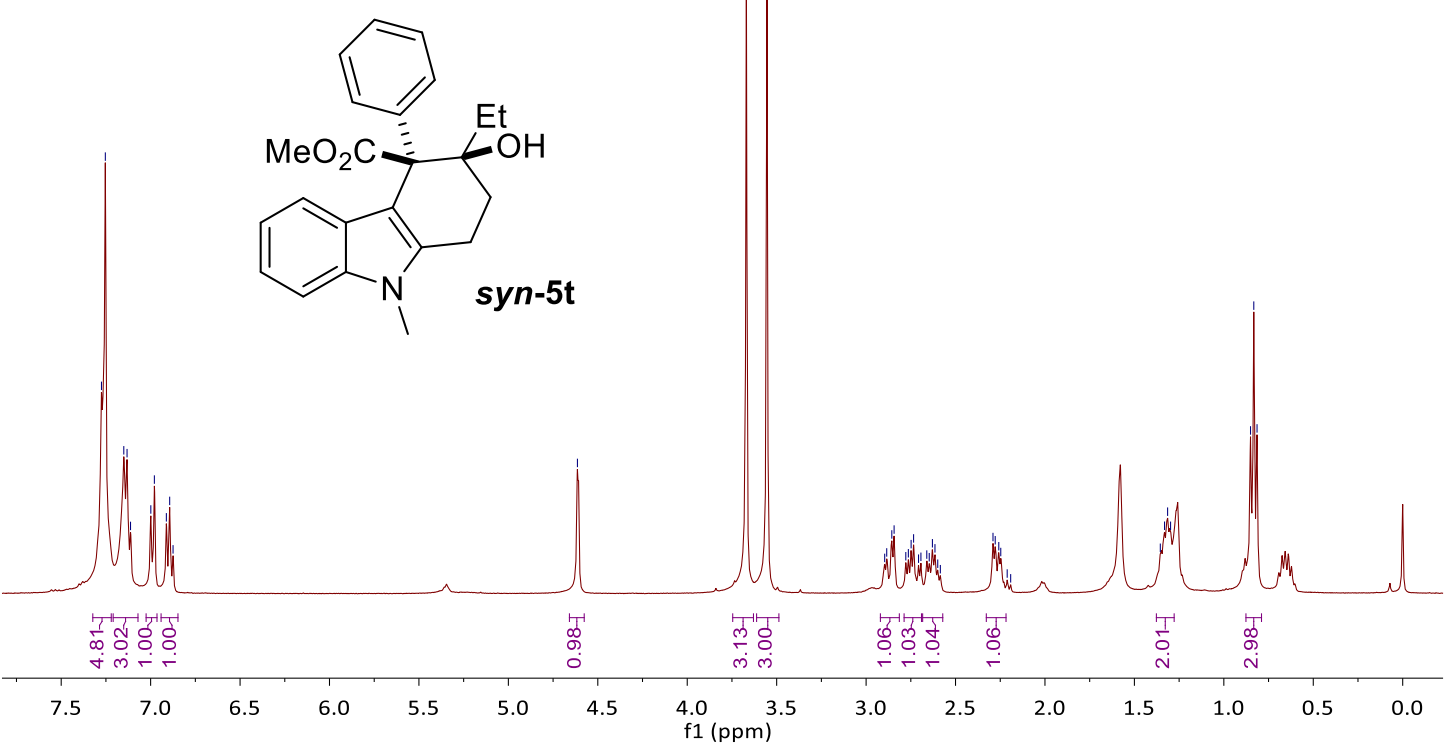

${ }^{1} \mathrm{H}$ NMR Spectrum of Compound $\mathbf{5 t}\left(400 \mathrm{MHz}, \mathrm{CDCl}_{3}\right)$.

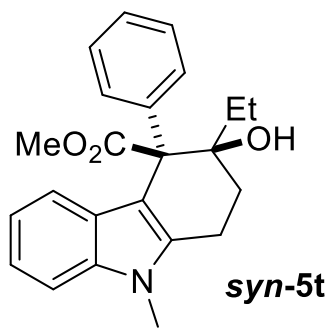

${ }^{13} \mathrm{C}$ NMR Spectrum of Compound $\mathbf{5 t}\left(100 \mathrm{MHz}, \mathrm{CDCl}_{3}\right)$. 


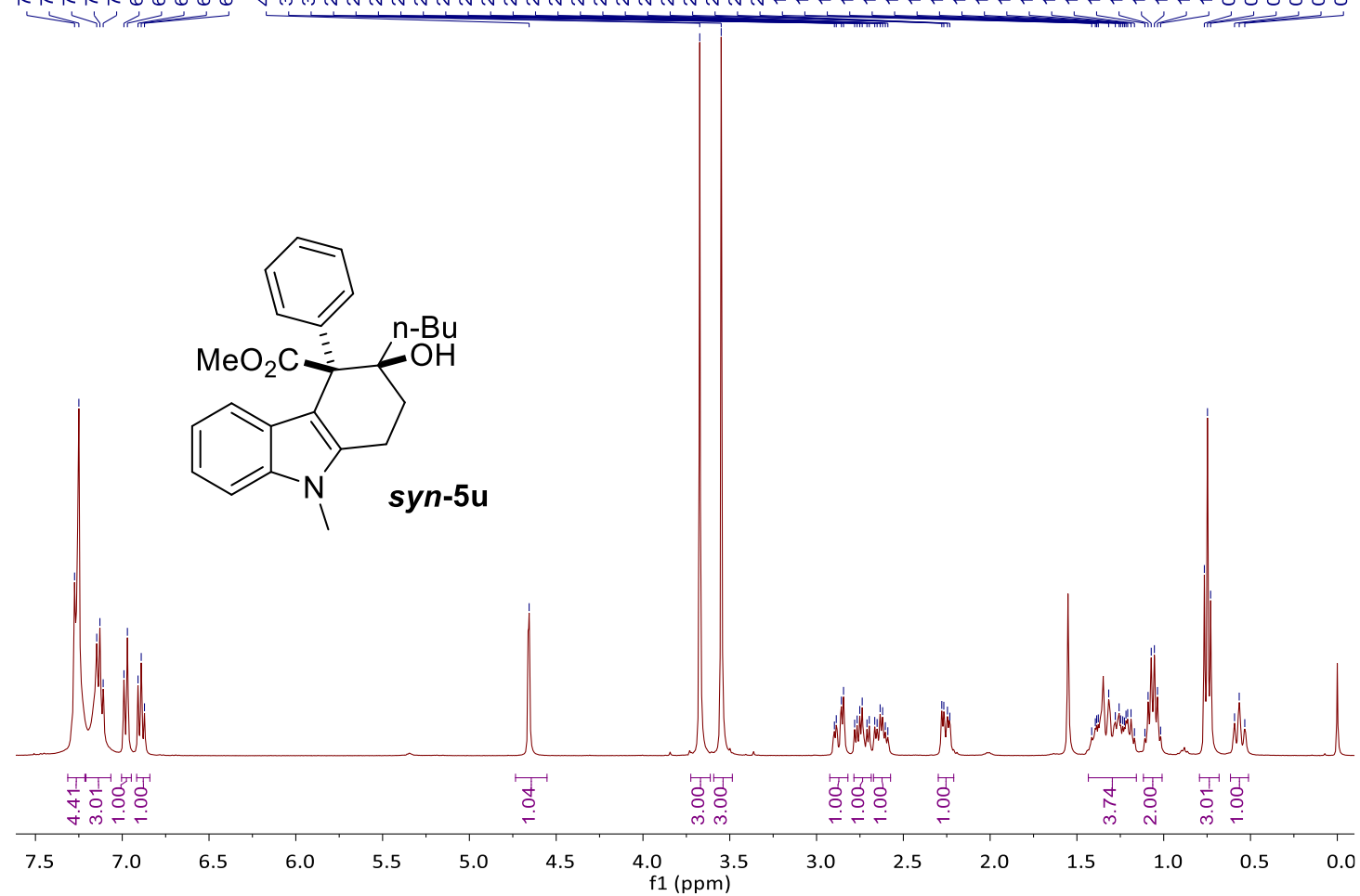

${ }^{1} \mathrm{H}$ NMR Spectrum of Compound $\mathbf{5 u}\left(400 \mathrm{MHz}, \mathrm{CDCl}_{3}\right)$.

$\stackrel{i}{\stackrel{2}{*}}$ $\underbrace{m}$
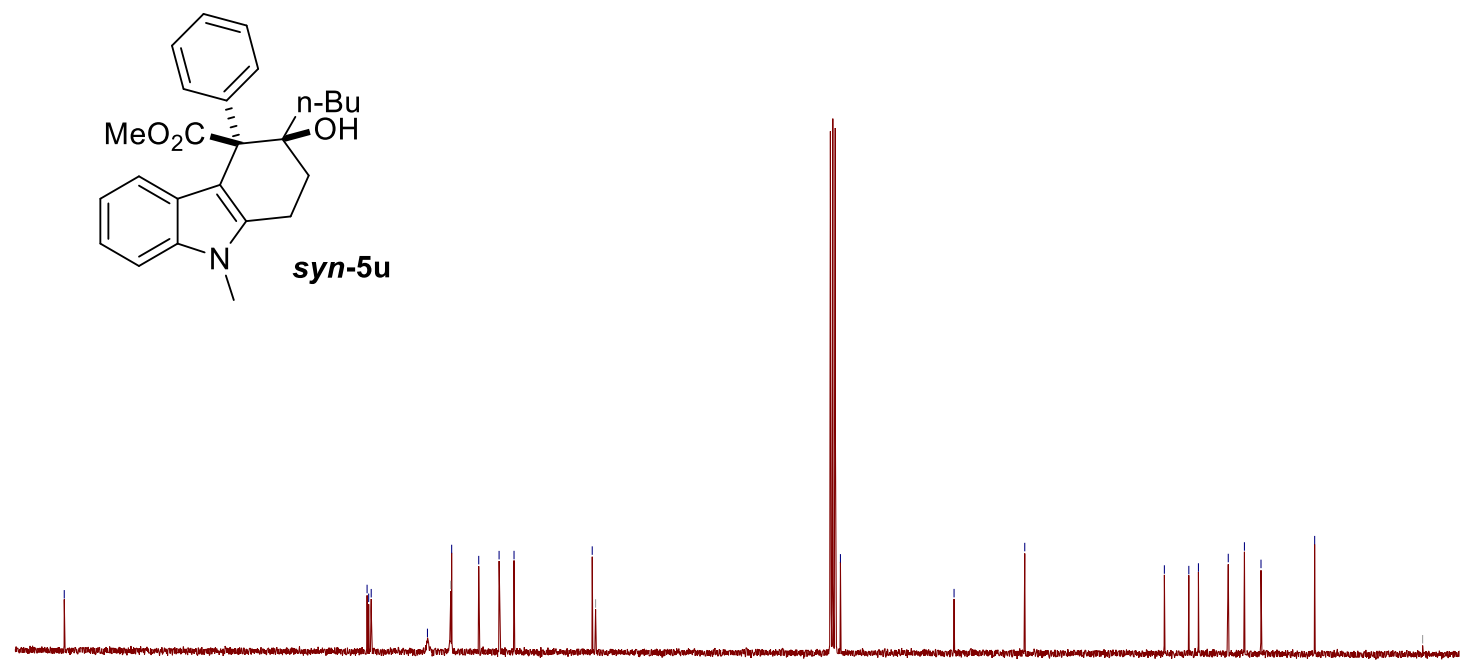

f1 (ppm)

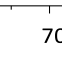

${ }^{13} \mathrm{C}$ NMR Spectrum of Compound $\mathbf{5 u}\left(100 \mathrm{MHz}, \mathrm{CDCl}_{3}\right)$. 


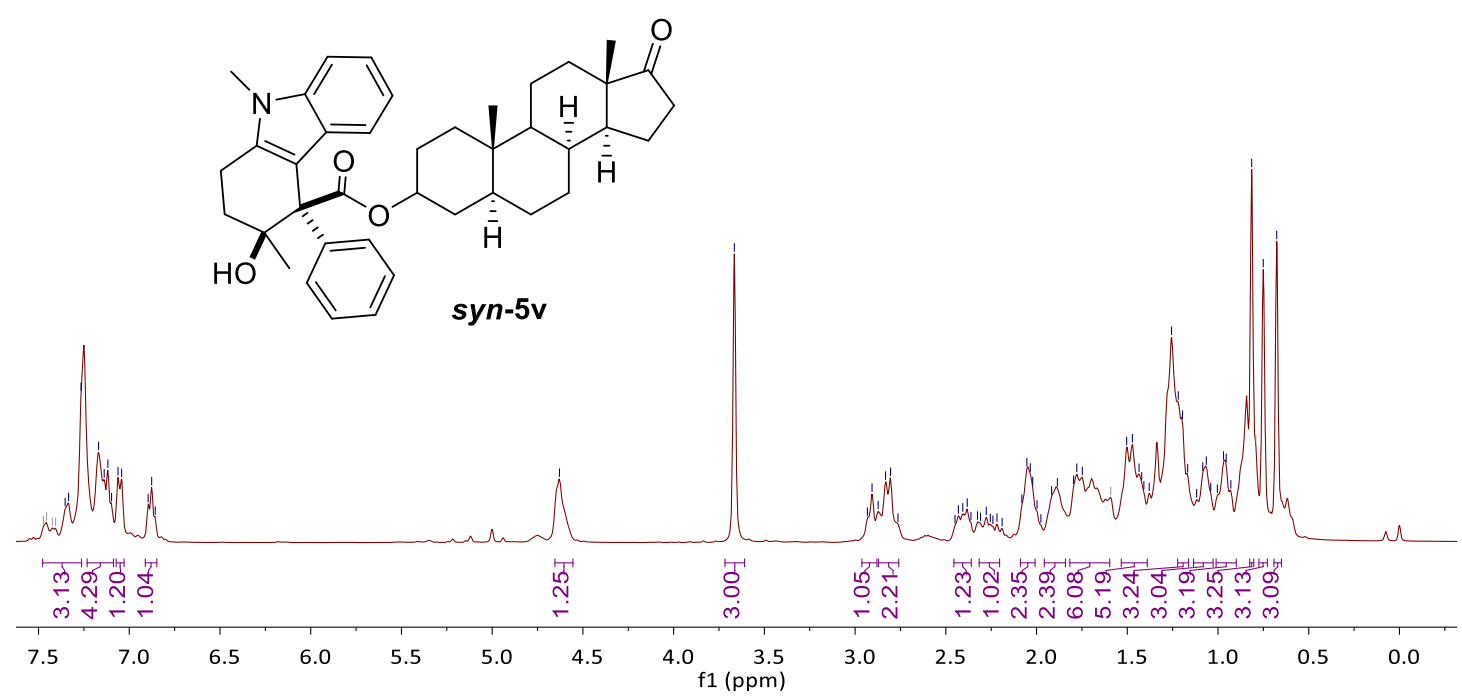

${ }^{1} \mathrm{H}$ NMR Spectrum of Compound $\mathbf{5 v}\left(400 \mathrm{MHz}, \mathrm{CDCl}_{3}\right)$.

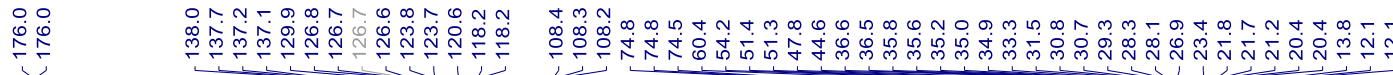

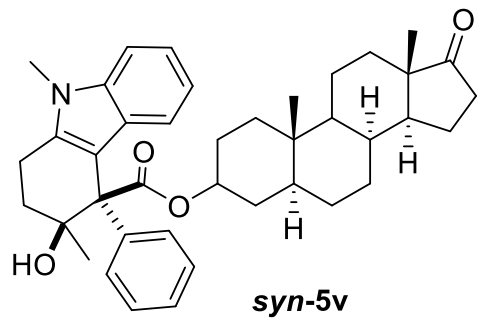

$\begin{array}{lllllllllllllllllll}180 & 170 & 160 & 150 & 140 & 130 & 120 & 110 & 100 \underset{\mathrm{f} 1(\mathrm{ppm})}{90} & 80 & 70 & 60 & 50 & 40 & 30 & 20 & 10 & 0\end{array}$

${ }^{13} \mathrm{C}$ NMR Spectrum of Compound $\mathbf{5 v}\left(100 \mathrm{MHz}, \mathrm{CDCl}_{3}\right)$. 


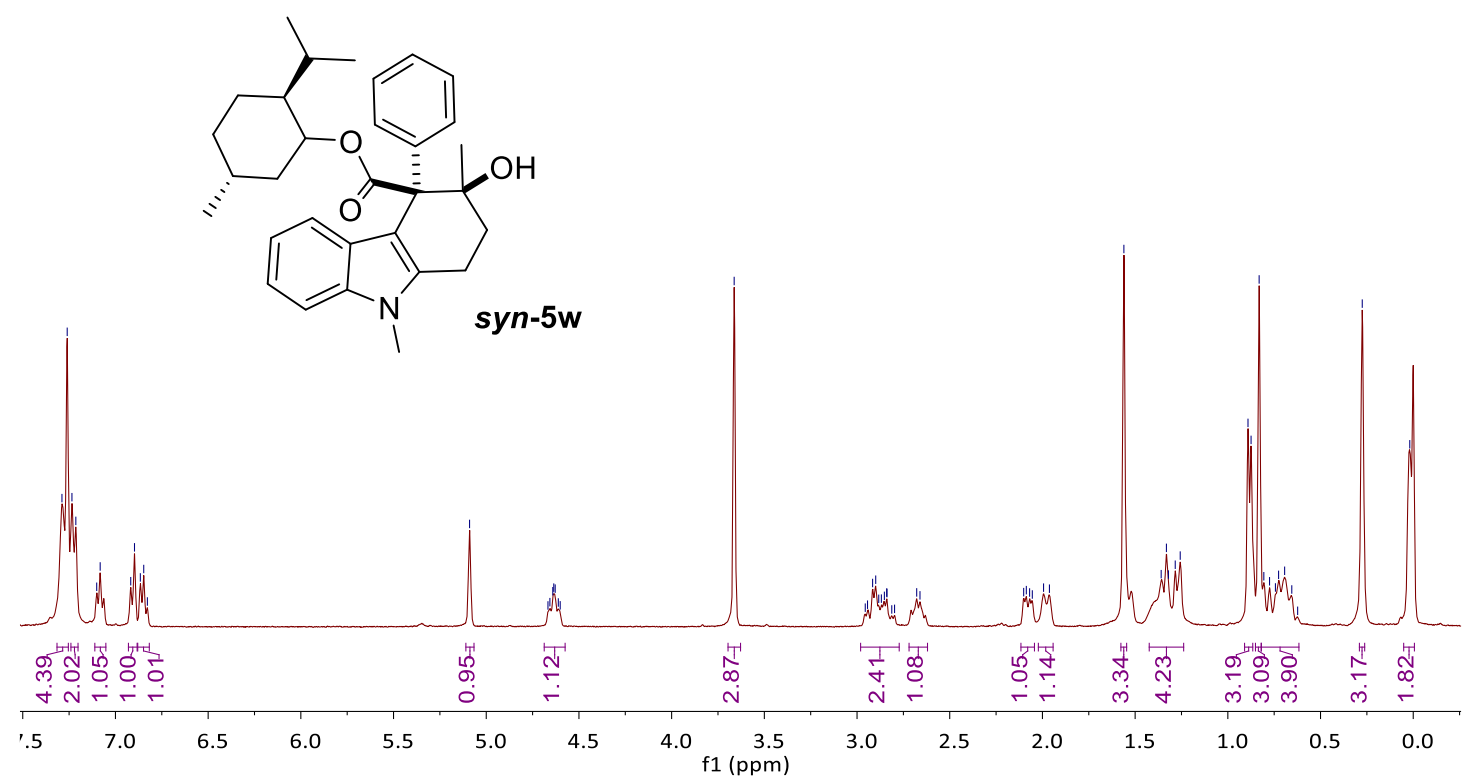

${ }^{1} \mathrm{H}$ NMR Spectrum of Compound $5 \mathbf{w}\left(400 \mathrm{MHz}, \mathrm{CDCl}_{3}\right)$.

$\stackrel{\substack{0 \\ \stackrel{0}{5}}}{+}$

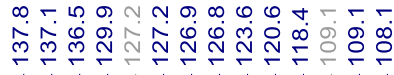

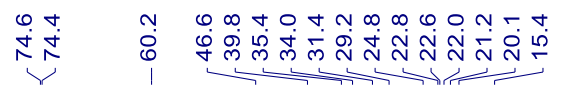

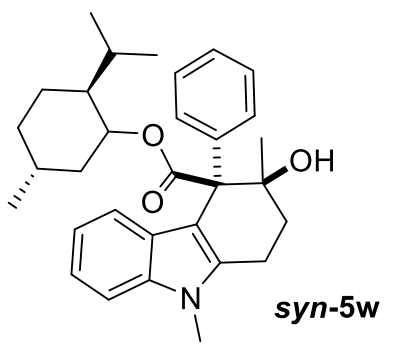

180

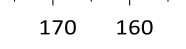

150

${ }^{13} \mathrm{C}$ NMR Spectrum of Compound $\mathbf{5 w}\left(100 \mathrm{MHz}, \mathrm{CDCl}_{3}\right)$. 

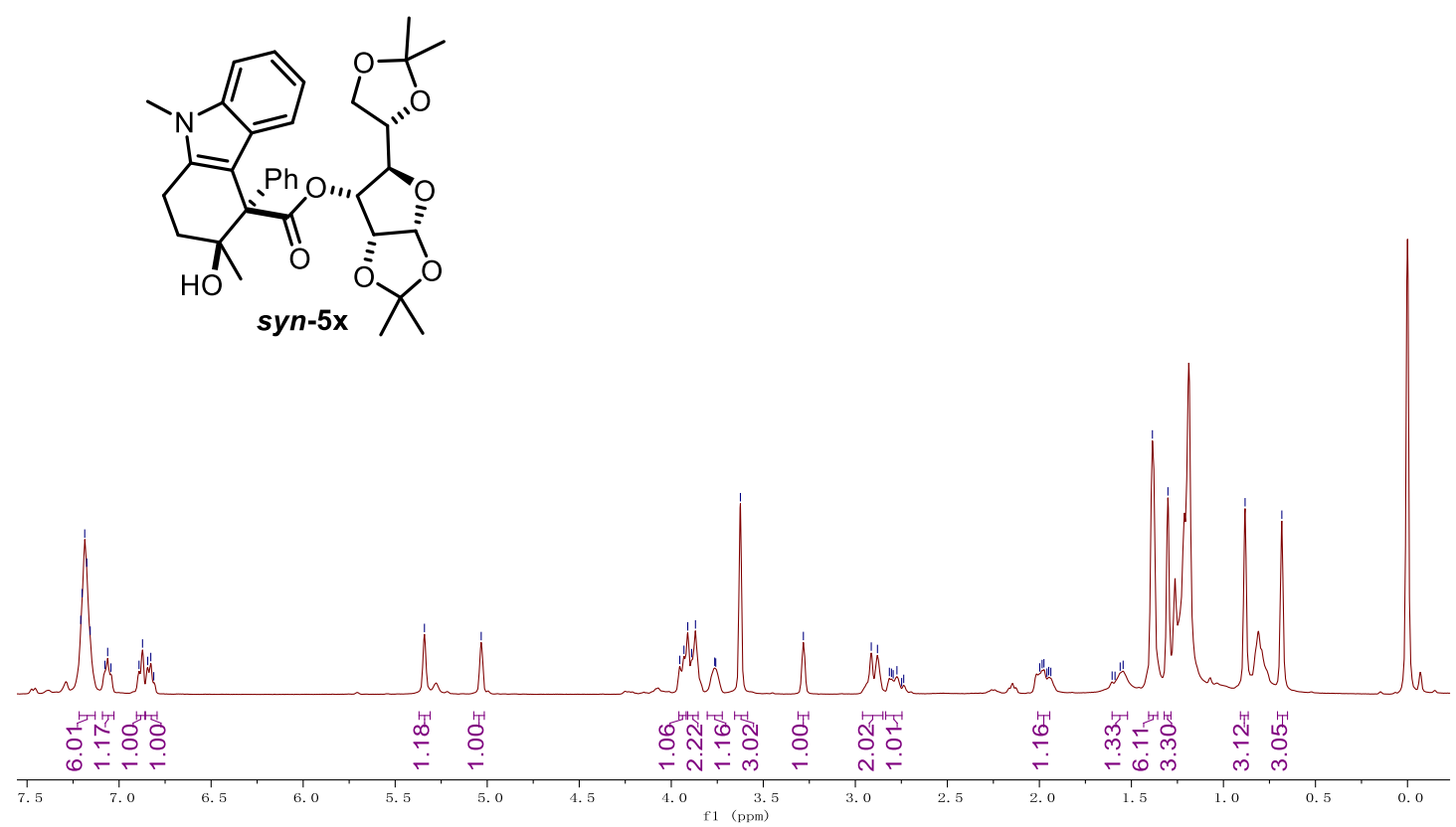

${ }^{1} \mathrm{H}$ NMR Spectrum of Compound $\mathbf{5 x}\left(400 \mathrm{MHz}, \mathrm{CDCl}_{3}\right)$.

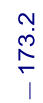

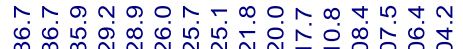

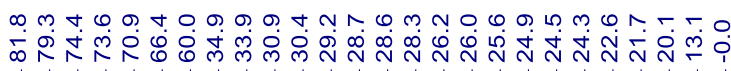<smiles>Cn1c2c(c3ccccc31)[C@](C(=O)O)(C(=O)O[C@H]1[C@@H]3OC(C)(C)O[C@H]3O[C@@H]1[C@H]1COC(C)(C)O1)C(C)(O)CC2</smiles>

syn-5x $X^{0}$

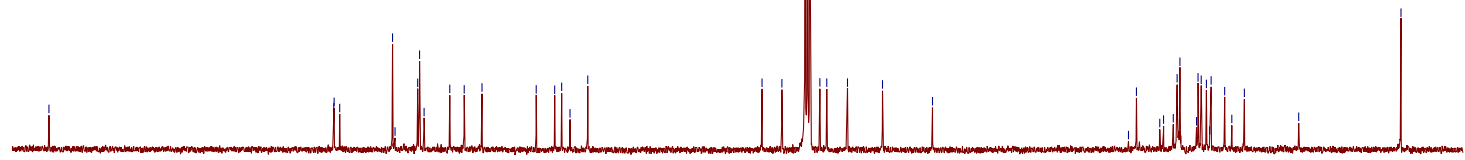

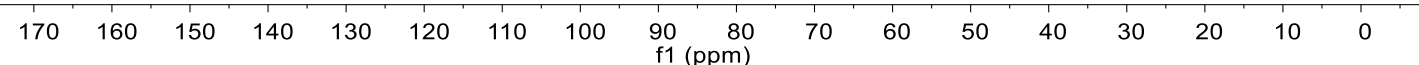

${ }^{13} \mathrm{C}$ NMR Spectrum of Compound $\mathbf{5 x}\left(100 \mathrm{MHz}, \mathrm{CDCl}_{3}\right)$. 

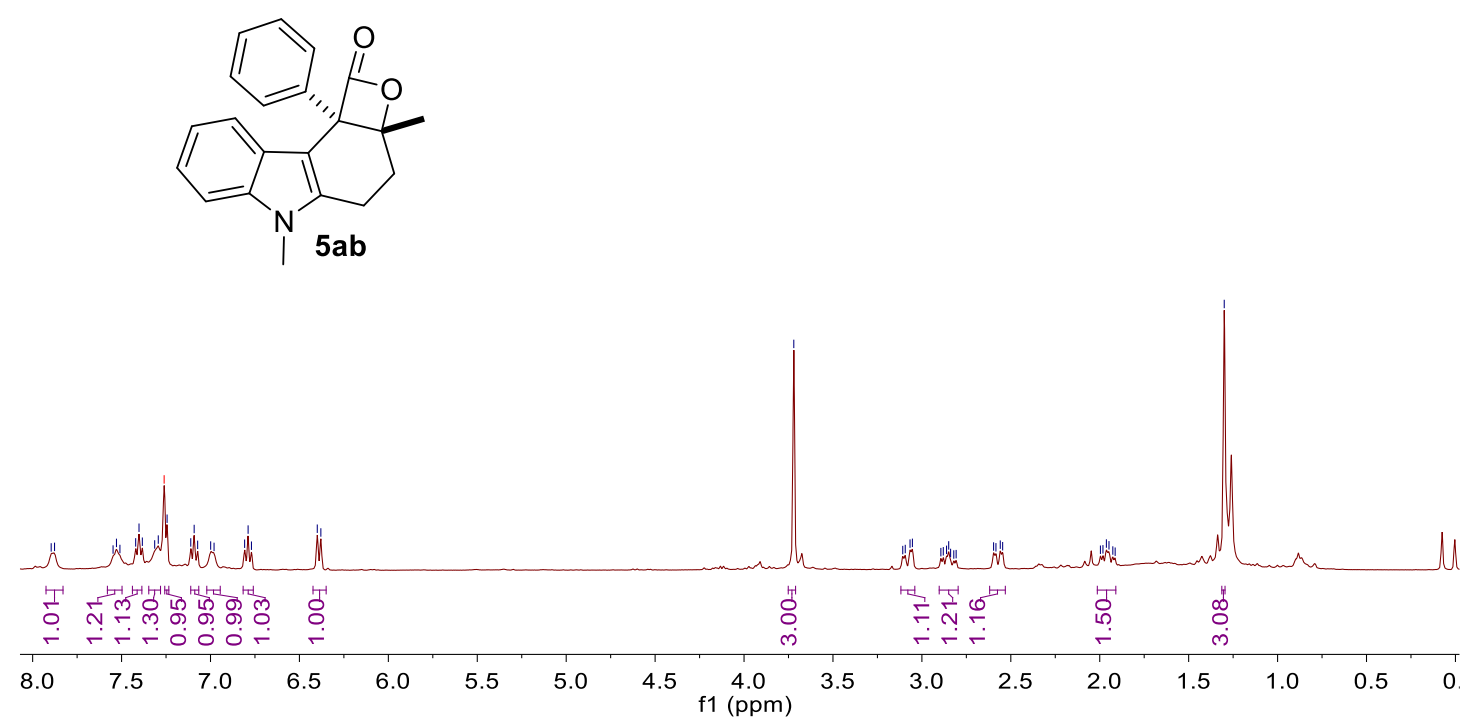

${ }^{1} \mathrm{H}$ NMR Spectrum of Compound $\mathbf{5 a b}\left(400 \mathrm{MHz}, \mathrm{CDCl}_{3}\right)$.

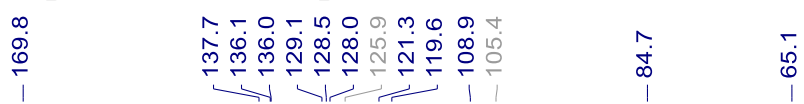

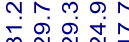

m N
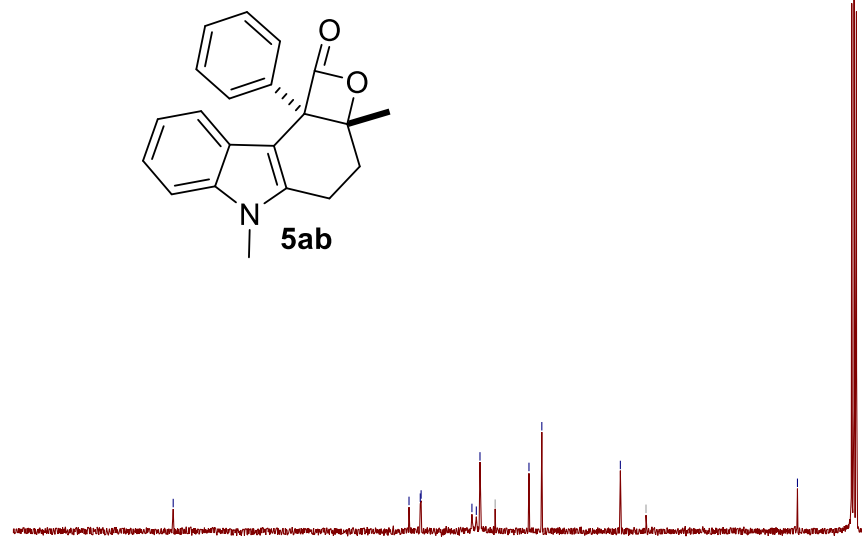

$\begin{array}{llllllllllllllllllll}190 & 180 & 170 & 160 & 150 & 140 & 130 & 120 & 110 & \begin{array}{r}100 \\ \mathrm{f} 1(\mathrm{ppm})\end{array} & 80 & 70 & 60 & 50 & 40 & 30 & 20 & 10 & 0\end{array}$

${ }^{13} \mathrm{C}$ NMR Spectrum of Compound $\mathbf{5 a b}\left(100 \mathrm{MHz}, \mathrm{CDCl}_{3}\right)$. 


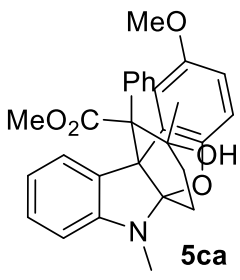

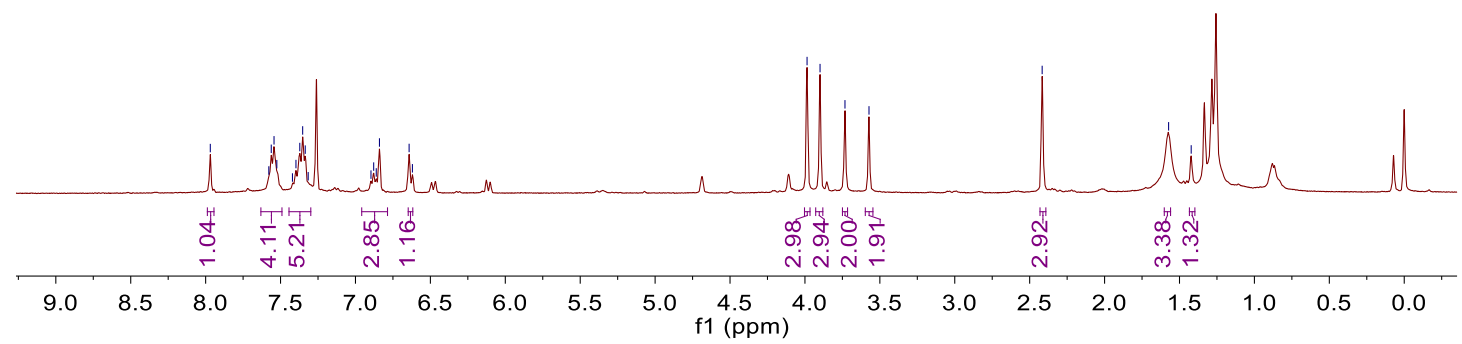

${ }^{1} \mathrm{H}$ NMR Spectrum of Compound 5ca $\left(400 \mathrm{MHz}, \mathrm{CDCl}_{3}\right)$.

$\stackrel{\substack{0 \\ \hdashline}}{\substack{0 \\ 0}}$

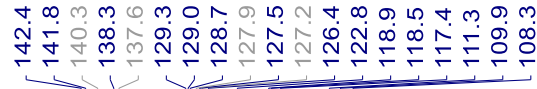

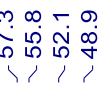

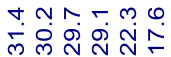
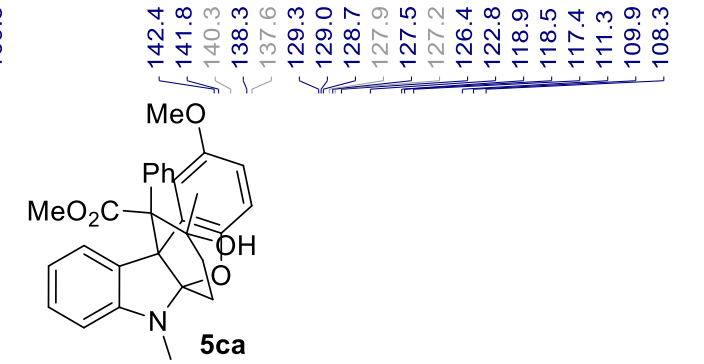

1

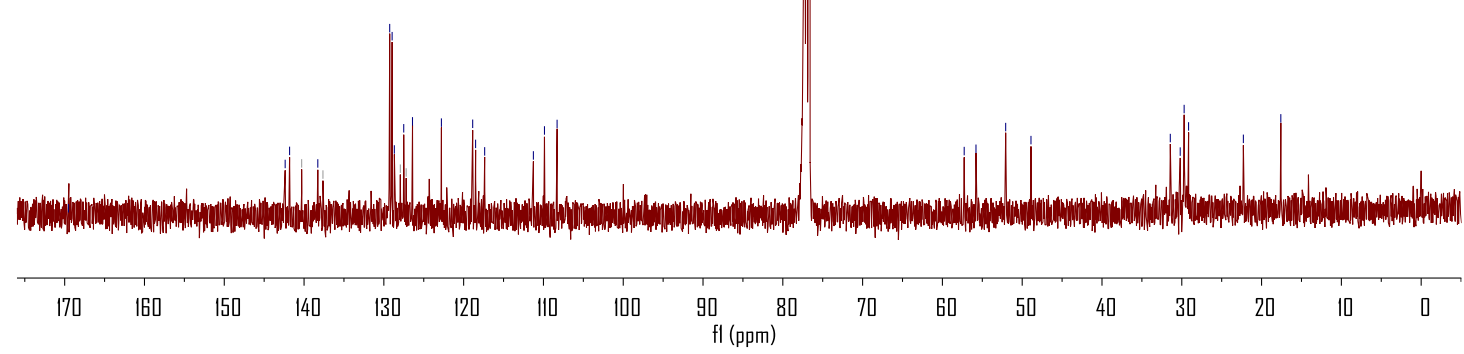

${ }^{13} \mathrm{C}$ NMR Spectrum of Compound $\mathbf{5 c a}\left(100 \mathrm{MHz}, \mathrm{CDCl}_{3}\right)$. 

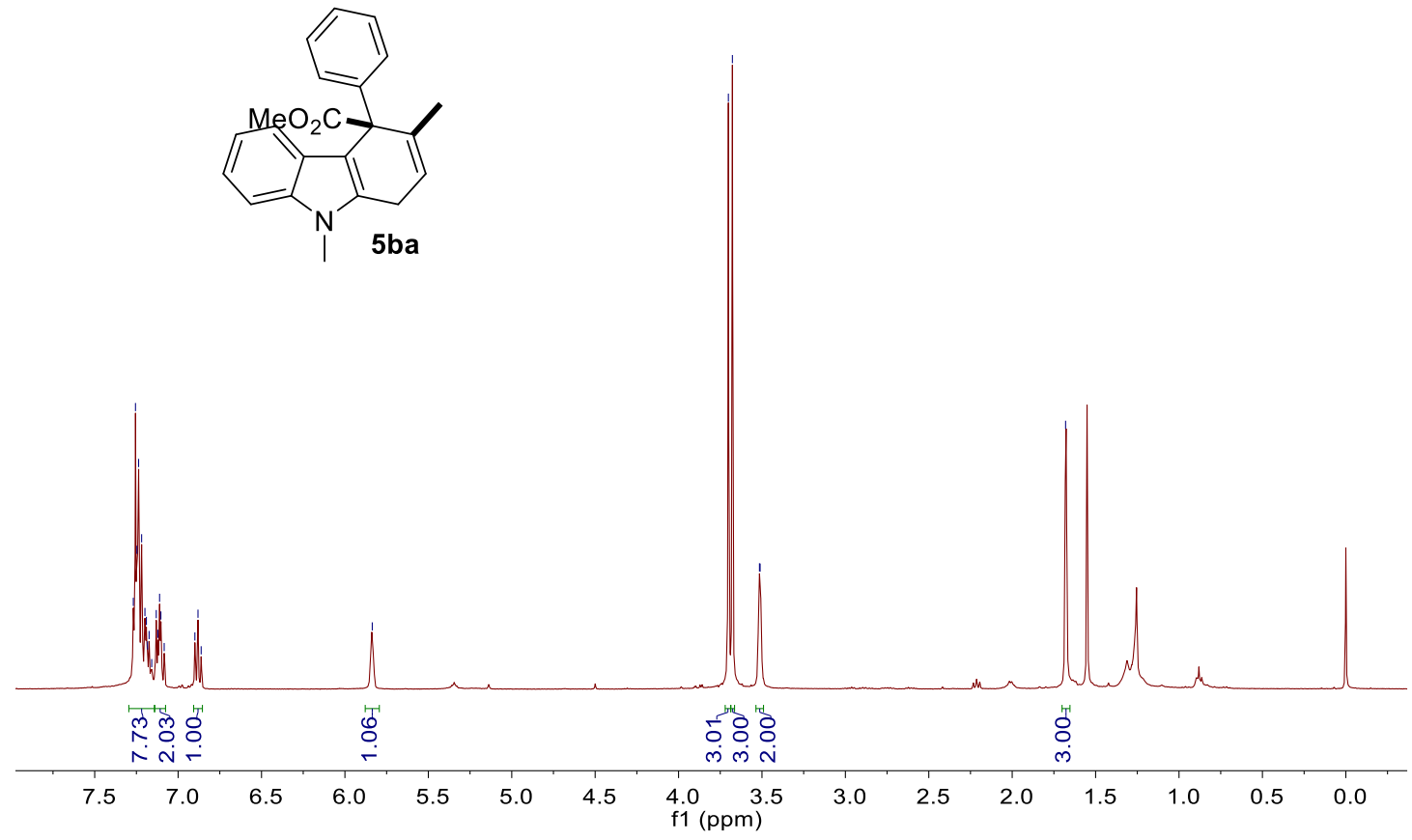

${ }^{1} \mathrm{H}$ NMR Spectrum of Compound $5 \mathbf{b a}\left(400 \mathrm{MHz}, \mathrm{CDCl}_{3}\right)$.

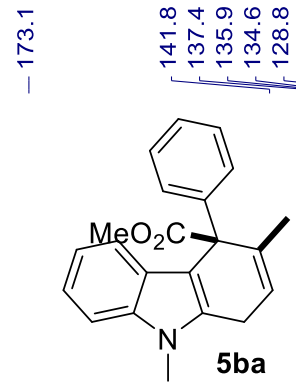

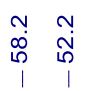

กั่

No

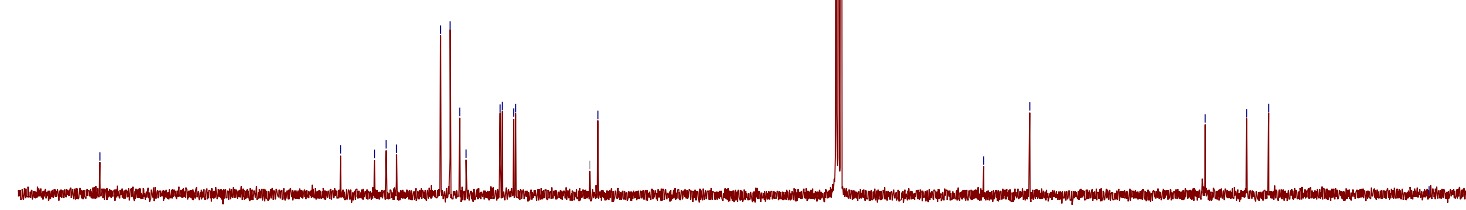

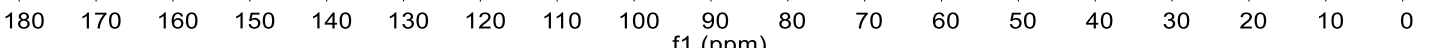

${ }^{13} \mathrm{C}$ NMR Spectrum of Compound $\mathbf{5 b a}\left(100 \mathrm{MHz}, \mathrm{CDCl}_{3}\right)$. 

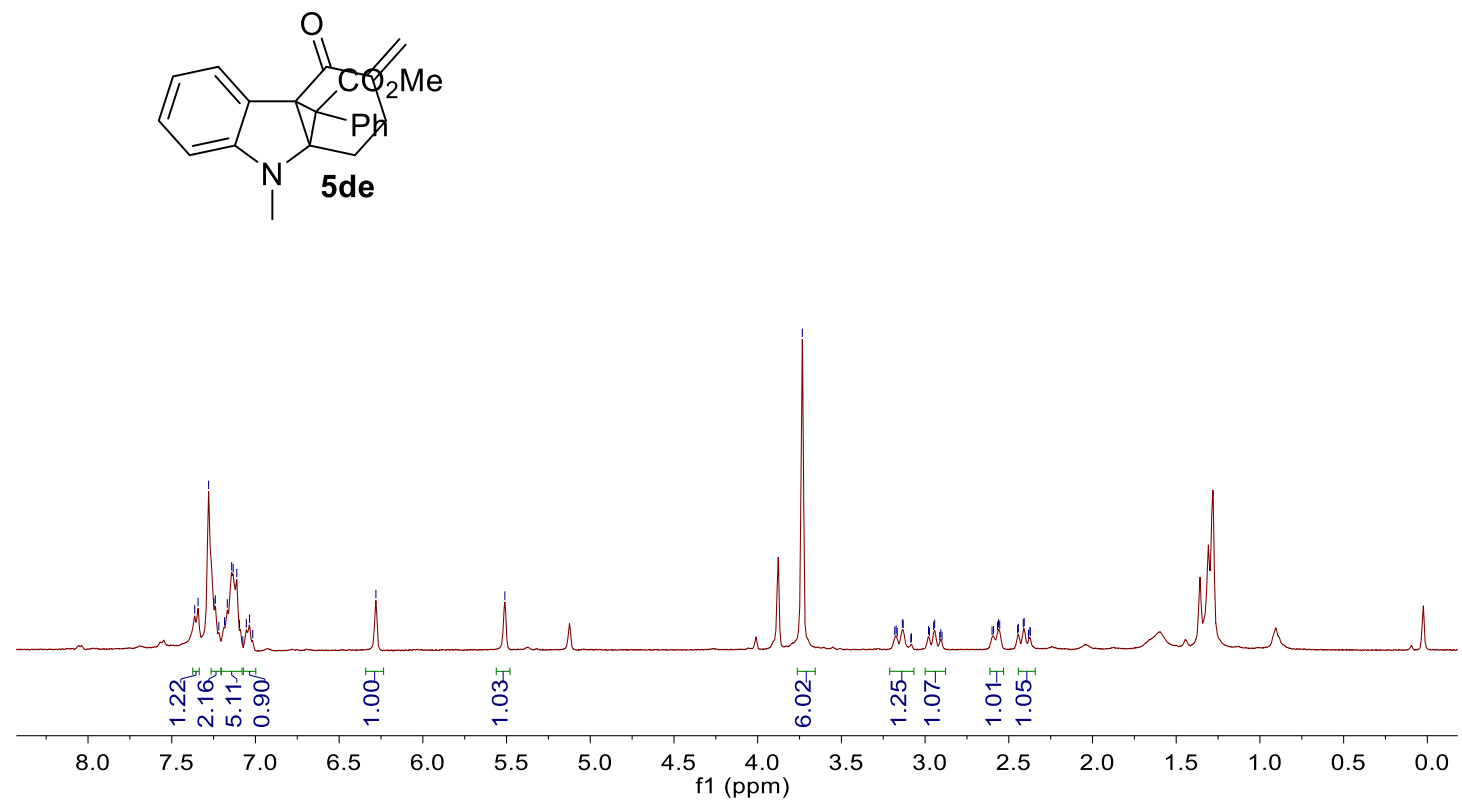

${ }^{1} \mathrm{H}$ NMR Spectrum of Compound 5de (400MHz, $\left.\mathrm{CDCl}_{3}\right)$.

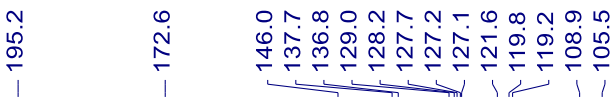

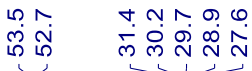

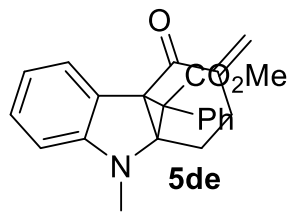

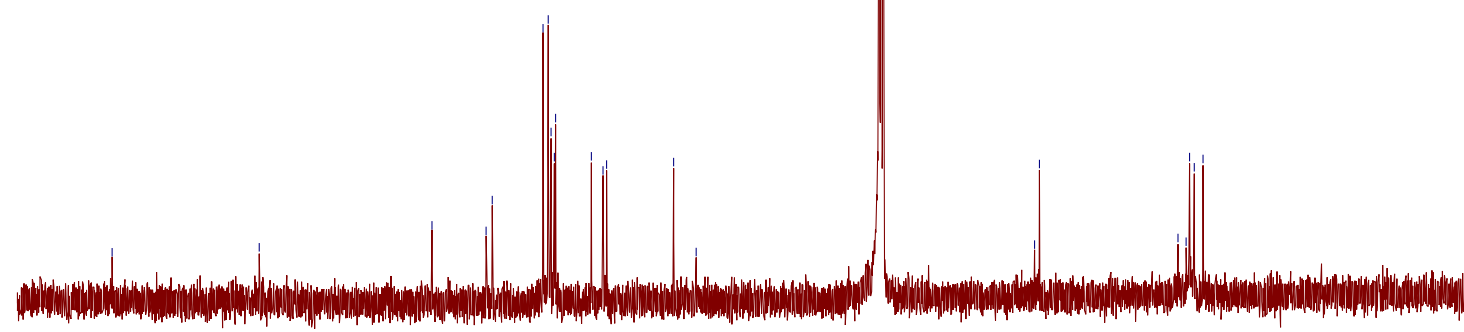

$\begin{array}{llllllllllllllllllllllll}200 & 190 & 180 & 170 & 160 & 150 & 140 & 130 & 120 & 110 & 100 & 90 & 80 & 70 & 60 & 50 & 40 & 30 & 20 & 10 & 0 & -1 \mathrm{C}\end{array}$

${ }^{13} \mathrm{C}$ NMR Spectrum of Compound $\mathbf{5 d e}\left(100 \mathrm{MHz}, \mathrm{CDCl}_{3}\right)$. 


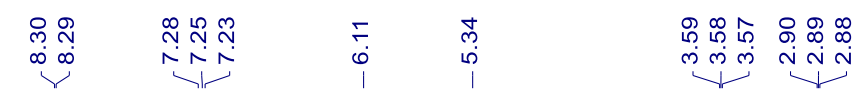<smiles>C=C1CCc2c(c3ccccc3n2C(C)(F)Cl)C1=O</smiles>

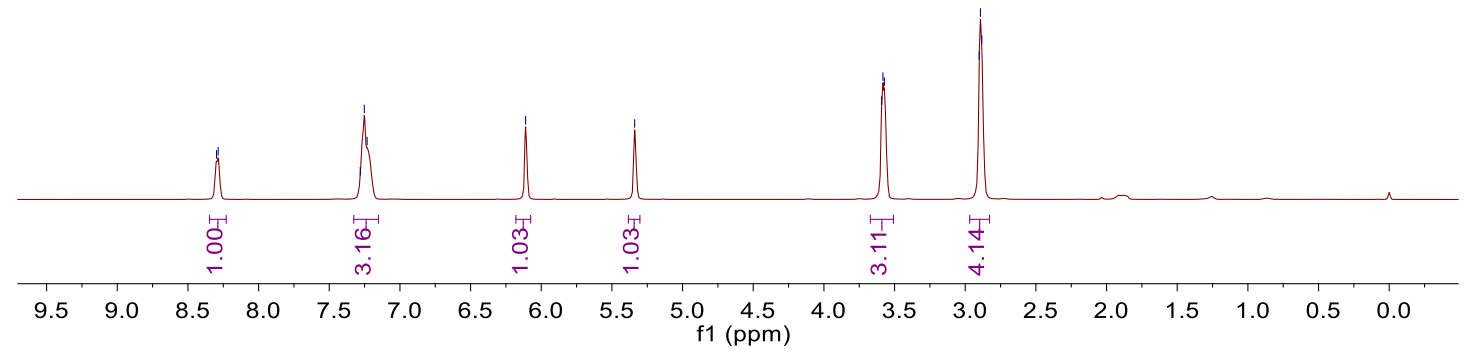

${ }^{1} \mathrm{H}$ NMR Spectrum of Compound $\mathbf{5 d d}\left(400 \mathrm{MHz}, \mathrm{CDCl}_{3}\right)$.
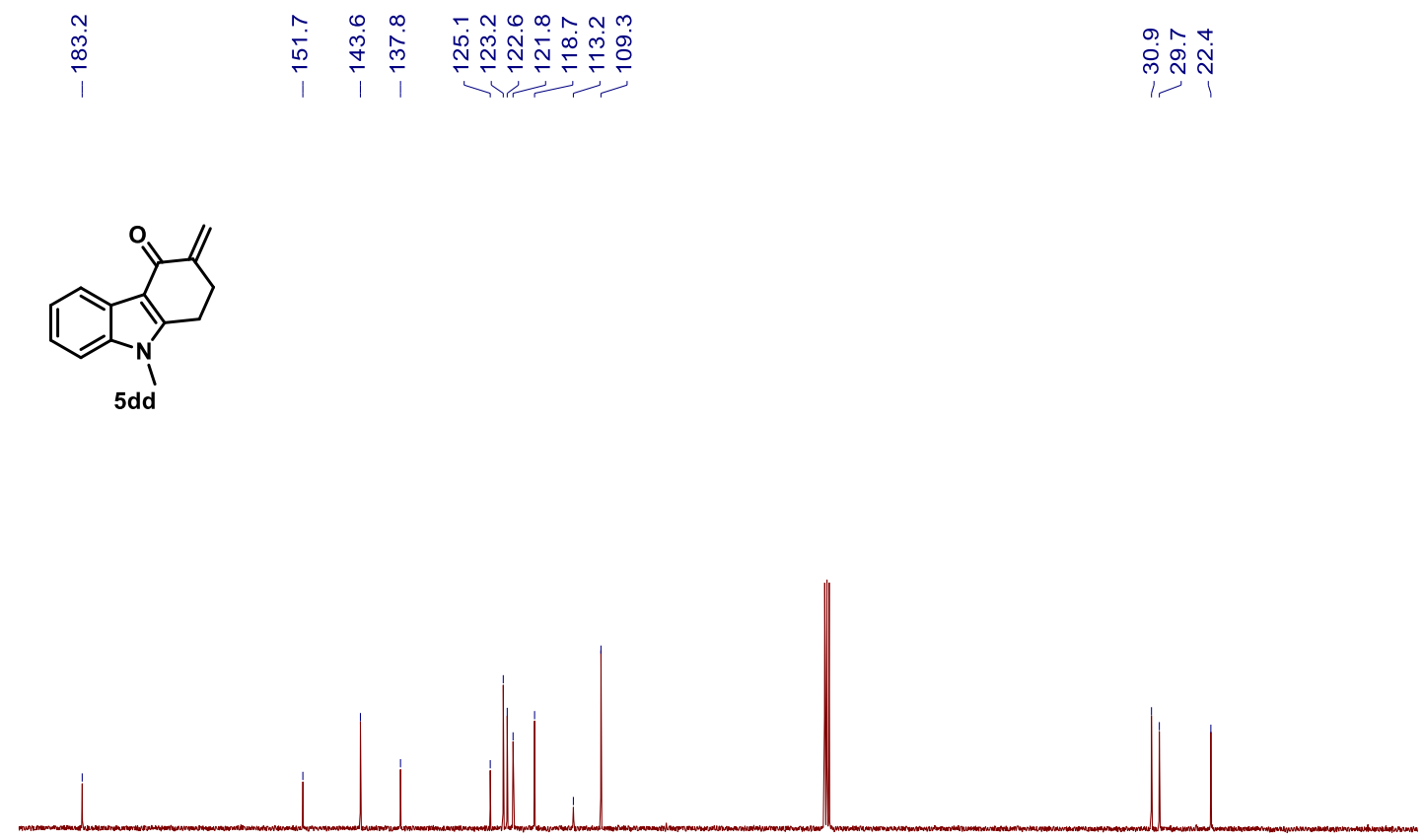

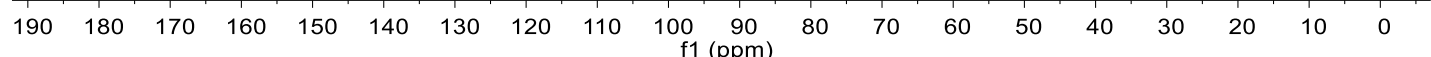

${ }^{13} \mathrm{C}$ NMR Spectrum of Compound $5 \mathbf{d d}\left(100 \mathrm{MHz}, \mathrm{CDCl}_{3}\right)$. 

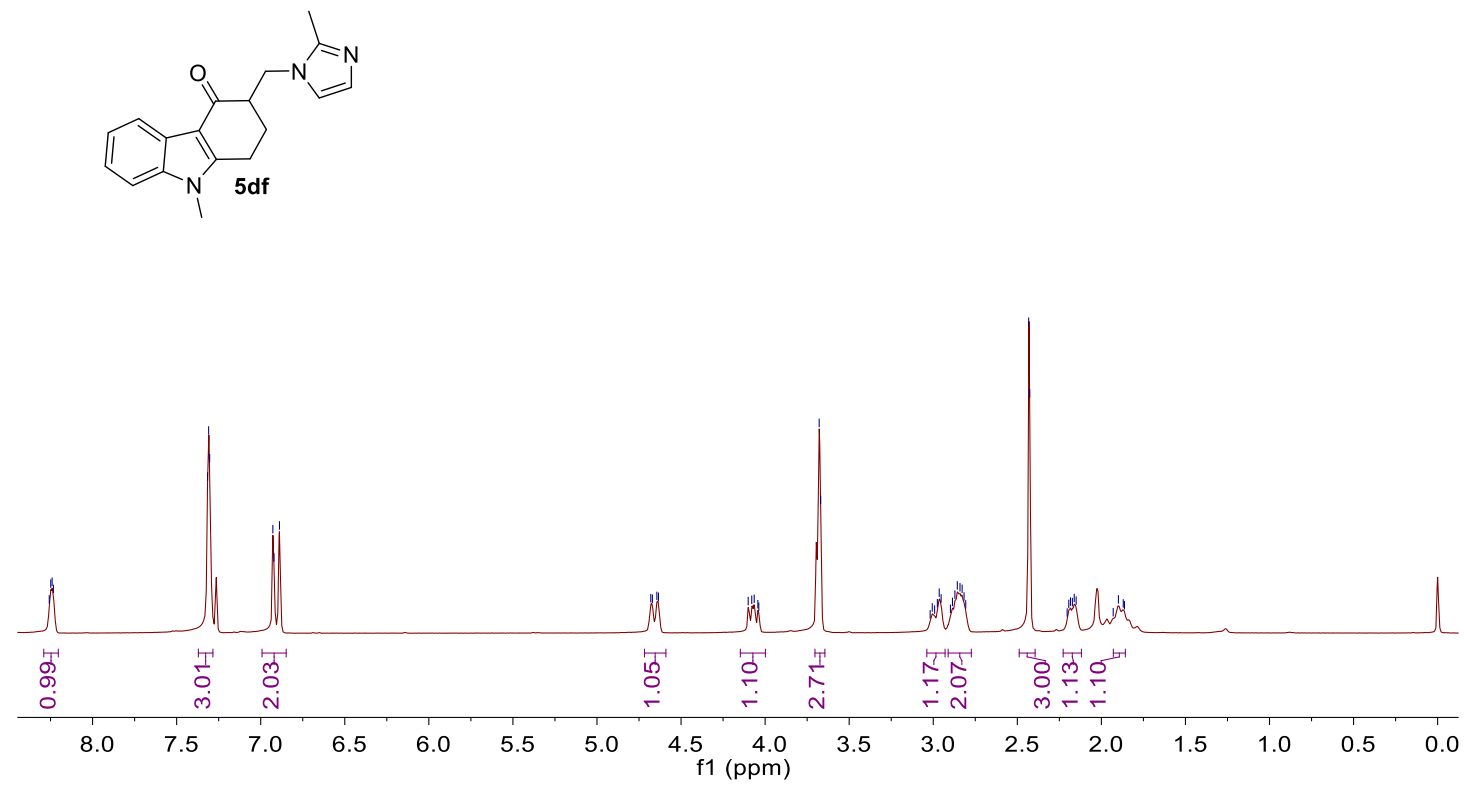

${ }^{1} \mathrm{H}$ NMR Spectrum of Compound $\mathbf{5 d f}\left(400 \mathrm{MHz}, \mathrm{CDCl}_{3}\right)$.

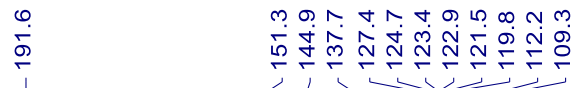

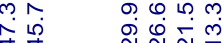

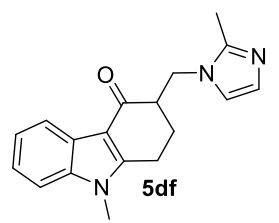

$\begin{array}{lllllllllllllllllllllll}210 & 200 & 190 & 180 & 170 & 160 & 150 & 140 & 130 & 120 & 110 & 100 & 90 & 80 & 70 & 60 & 50 & 40 & 30 & 20 & 10 & 0 & -10\end{array}$

${ }^{13} \mathrm{C}$ NMR Spectrum of Compound $\mathbf{5 d f}\left(100 \mathrm{MHz}, \mathrm{CDCl}_{3}\right)$ 